\title{
Development of an Erosion Control Seed Mixture that Includes Native Species
}

\author{
George A. Hilvers
}

Follow this and additional works at: https://researchrepository.wvu.edu/etd

\section{Recommended Citation}

Hilvers, George A., "Development of an Erosion Control Seed Mixture that Includes Native Species" (2015). Graduate Theses, Dissertations, and Problem Reports. 5807.

https://researchrepository.wvu.edu/etd/5807

This Thesis is protected by copyright and/or related rights. It has been brought to you by the The Research Repository @ WVU with permission from the rights-holder(s). You are free to use this Thesis in any way that is permitted by the copyright and related rights legislation that applies to your use. For other uses you must obtain permission from the rights-holder(s) directly, unless additional rights are indicated by a Creative Commons license in the record and/ or on the work itself. This Thesis has been accepted for inclusion in WVU Graduate Theses, Dissertations, and Problem Reports collection by an authorized administrator of The Research Repository @ WVU. For more information, please contact researchrepository@mail.wvu.edu. 


\title{
Development of an Erosion Control Seed Mixture that Includes Native Species
}

\section{George A. Hilvers}

Thesis submitted to the

Benjamin M. Statler College of Engineering and Mineral Resources

at West Virginia University

in Partial fulfillment of the requirements for the degree of

Master of Science in

Civil and Environmental Engineering

Dr. Leslie Hopkinson, Ph.D., Chair

Dr. Avinash Unikrishnan, Ph.D.

Dr. Yoo Jung Yoon, Ph.D.

Department of Civil and Environmental Engineering

\author{
Morgantown, West Virginia \\ 2015
}

Keywords: Native seed mixtures, native seed species, roadside seed mixtures, erosion control,

roadside revegetation, roadside vegetation 


\section{Abstract \\ Development of an Erosion Control Seed Mixture that Includes Native Species \\ George A. Hilvers}

The West Virginia Divisions of Highways (WVDOH) utilizes the establishment of grass cover as a temporary and permanent management practice to mediate the effects of erosion on highway construction sites. Current West Virginia regulations include five seed mixtures for permanent applications that include combinations of nine species. Six of these species are identified as invasive, and all nine species are considered introduced or both native and introduced. This research developed alternative seed mixtures that included low threat or native species. The alternative seed mixtures were then evaluated in a field test. The research also examined seedbed preparation techniques, the performance of soil amendment and media products, and need for a high elevation specific mixture. Four plots were developed to test these variables over a 90 day period. The sites were monitored biweekly for percent cover. Sensors were in place to monitor precipitation, ambient air and soil temperatures, volumetric water content, and electrical conductivity. Four alternative mixtures (mowable areas, warm season, cool season and high elevation) performed as well or better than current WVDOH seed mixtures. Statistical analysis determined a high elevation specific mixture was not required to achieve adequate coverage at high elevations in West Virginia. After ninety days there were no significant differences among seedbed preparation techniques. 


\section{Acknowledgments}

I would like to take this time to thank the various individuals who aided in the success of this project. A special thanks and gratitude goes to my research advisor Dr. Leslie Hopkinson for her support and guidance in the development and evaluation of the project. I am greatly appreciative for her superlative role as my mentor, committee chairperson, and academic advisor.

Concerning the development of the seed mixtures, I would like to thank Mark B. Fiely from Ernst Conservation Seeds, Elizabeth A. Byers from the WVDNR, and Casey Shrader and Sonya Keith from the NRCS in Lexington, KY. Their assistance and vast knowledge allowed for the development of the proposed seed mixtures for the project. I would also like to thank Charlie Riling, Donald Williams, and Michael Pumphrey from the WVDOH for assisting in the experimental site selection and their assistance and evaluation throughout the project duration. Finally, I would like to thank Dr. Avinash Unnikrishnan and Dr. Yoo Jung Yoon for serving as committee members.

For product selection, assistance, and application, I would like to thank Profile Products, LLC for donating their time, expertise, and erosion control products to the benefit of the research. Verdyol was also much appreciated for their donation and product assistance for one of their soil amendment products. Also, I would like to thank Arnold's Custom Seeding, LLC for donating their time, knowledge, and equipment for the application of the various erosion control products used in this study. Without these companies and their amazing employees, this study would have not been possible or successful.

I would like to thank my parents, George and Cindy Hilvers, for their continued support and guidance. They have been a guiding force in promoting my greatest enjoyments in life. I would also like to thank Chelsea Wells for her support in my pursuit in advancing my higher education. 


\section{Grant Information}

The contents of this report reflect the views of the author who is responsible for the facts and accuracy of the data presented herein. The contents do not necessarily reflect the official views or policies of the State. This report does not constitute a standard, specification, or regulation. Trade or manufacturers' names which may appear herein are cited only because they are considered essential to the objectives of this report. The State of West Virginia does not endorse products or manufacturers. Prepared for the West Virginia Department of Transportation Division of Highways. 


\section{Table of Contents}

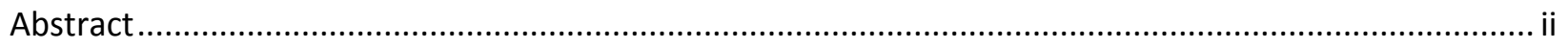

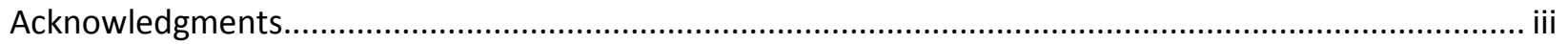

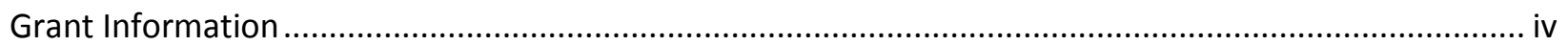

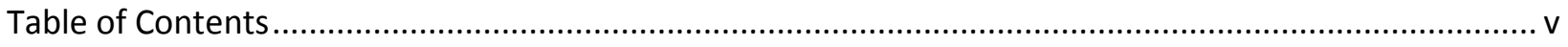

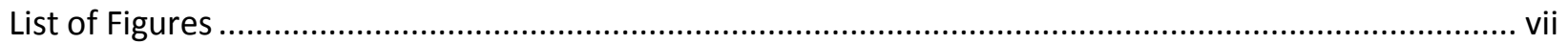

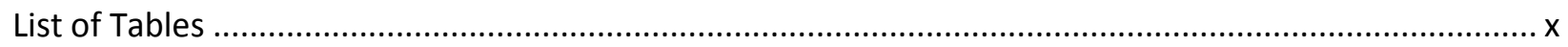

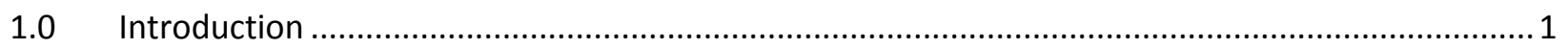

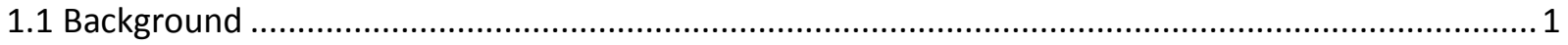

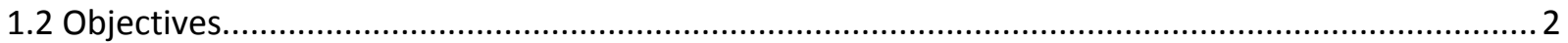

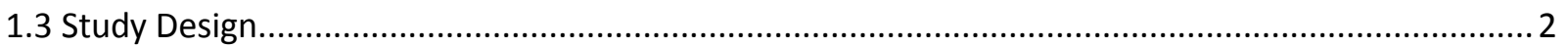

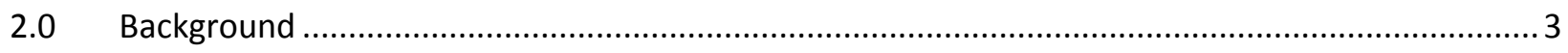

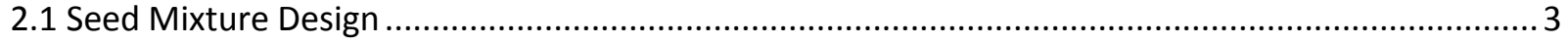

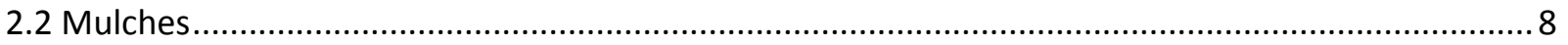

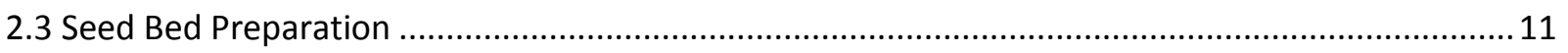

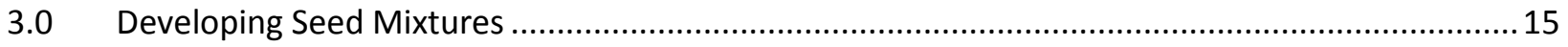

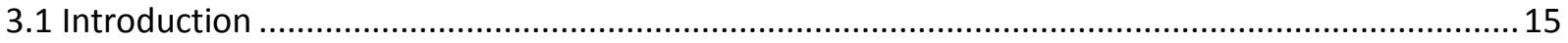

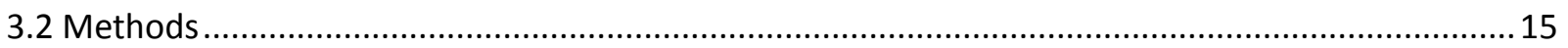

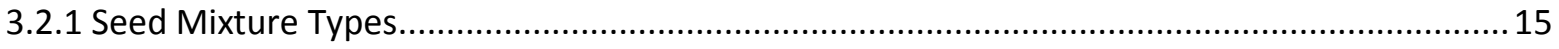

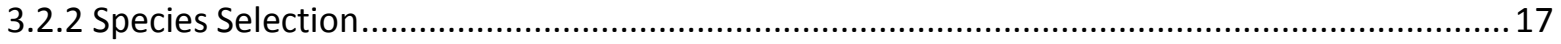

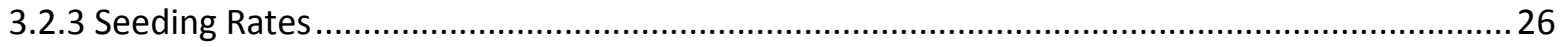

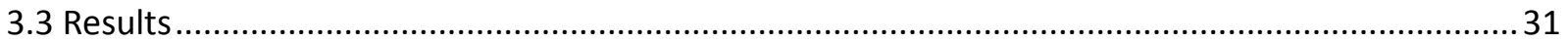

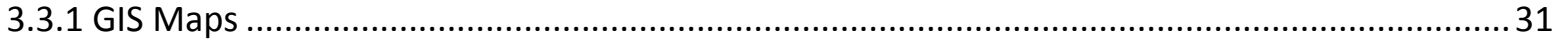

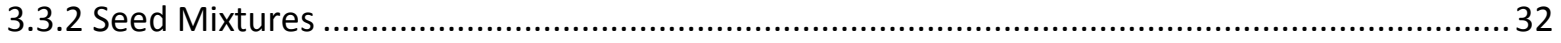

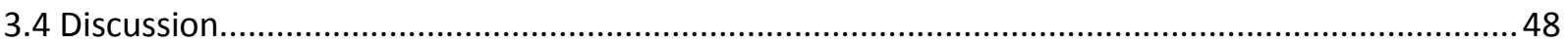

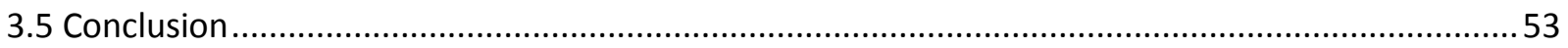

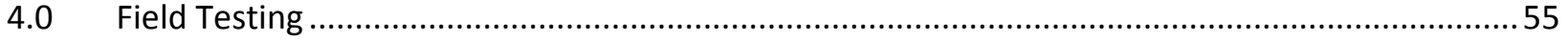

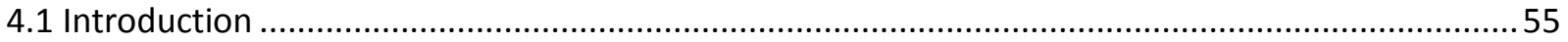

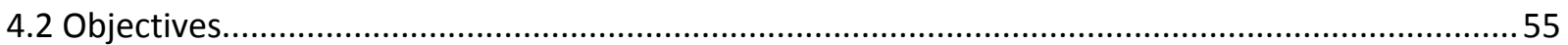




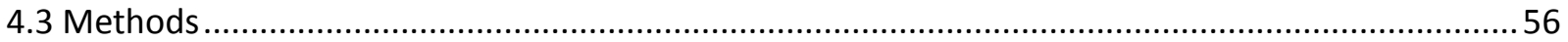

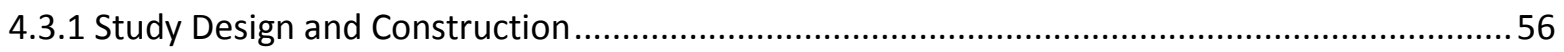

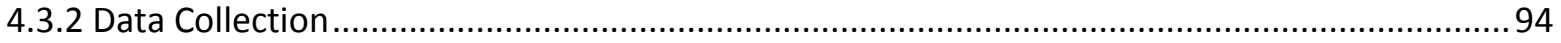

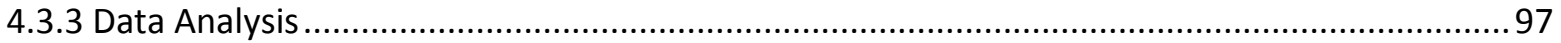

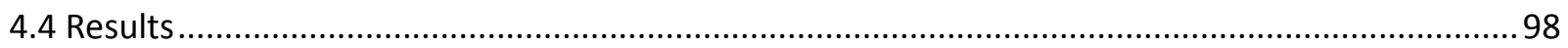

4.4.1 Objective 1: Comparison with current standard............................................................... 98

4.4.2 Objective 2: Evaluating high elevation needs .................................................................. 106

4.4.3 Objective 3: Examining seedbed preparation techniques ................................................... 115

4.4.4 Objective 4: Examining soil media and amendment products .......................................... 125

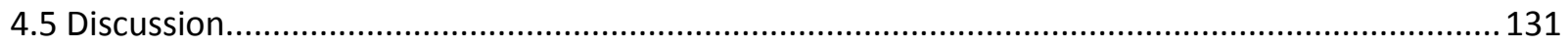

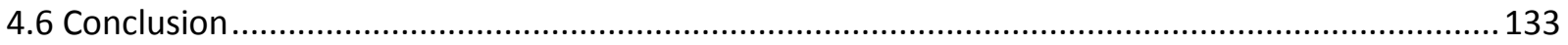

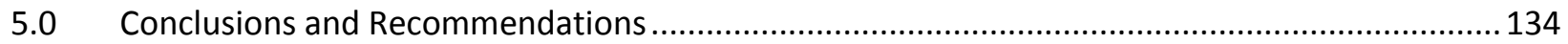

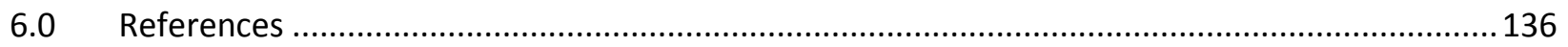

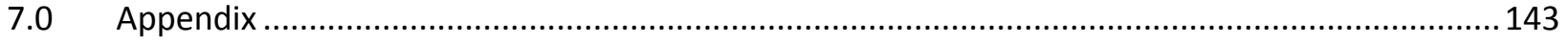

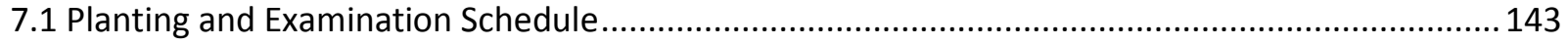

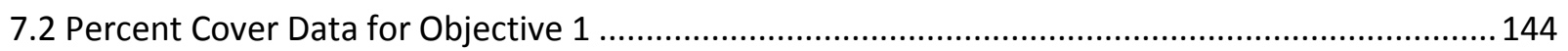

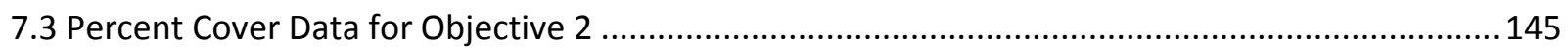

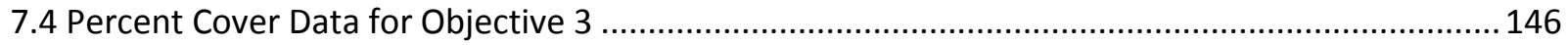

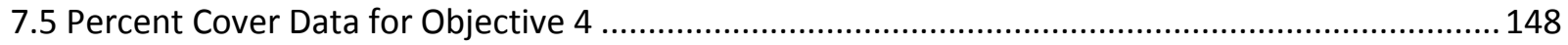




\section{List of Figures}

Figure 1: West Virginia Ecoregions

Figure 2: West Virginia Elevation (USGS NED 1/3 arc-second 2013 1x1 degree ArcGrid)

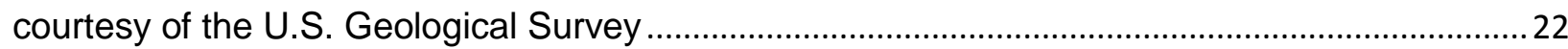

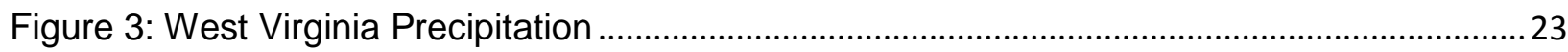

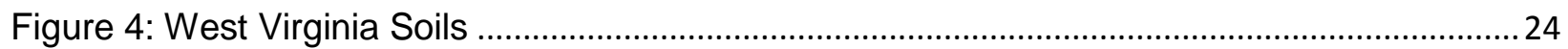

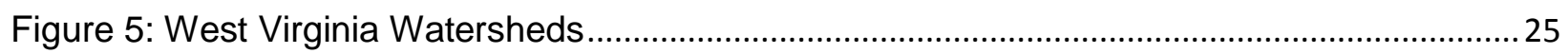

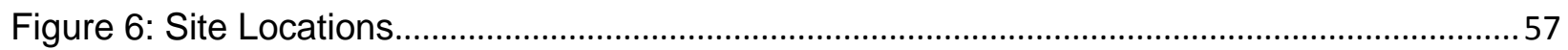

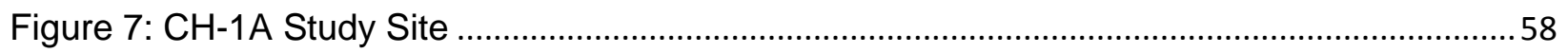

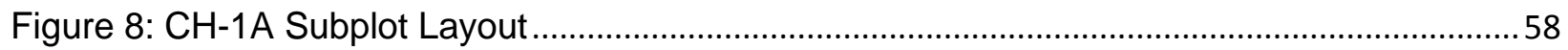

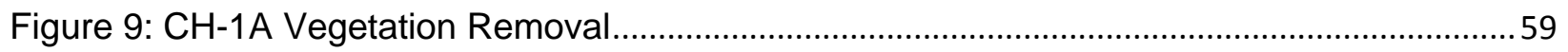

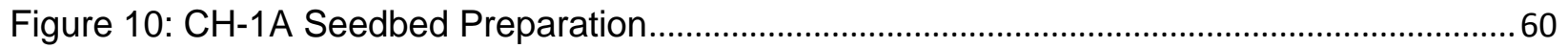

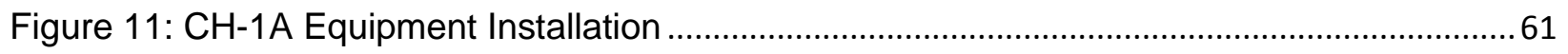

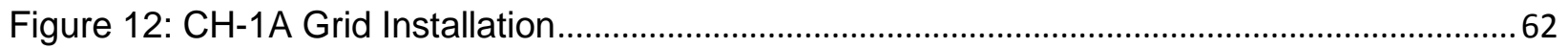

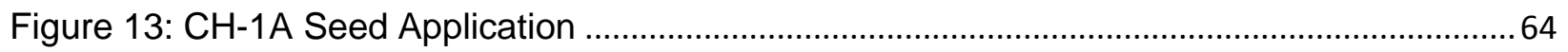

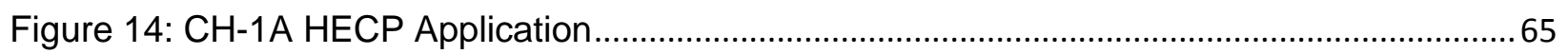

Figure 15: $\mathrm{CH}-1 \mathrm{~A}$ HECP (Photo by Profile Products, Adam Dibble/Matt Welch) .........................66

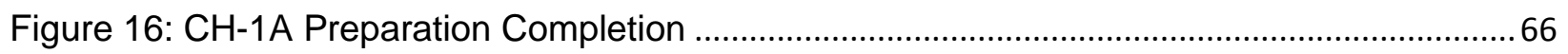

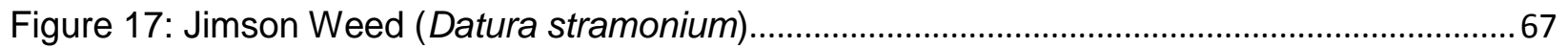

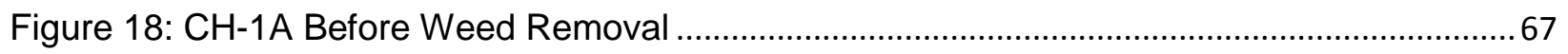

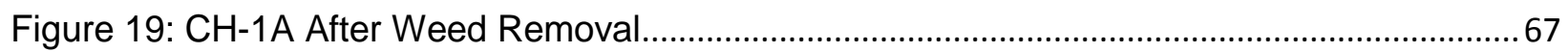

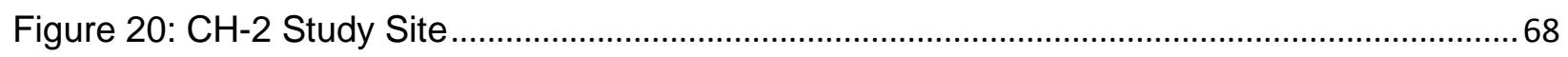

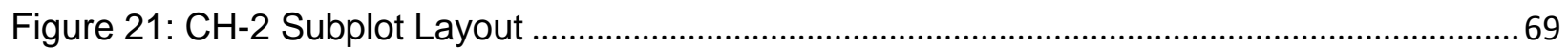

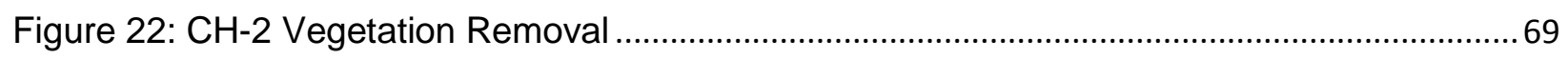

Figure 23: $\mathrm{CH}-2$ Fence Installation and Re-application of Plastic ................................................. 70

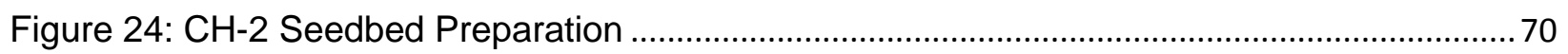

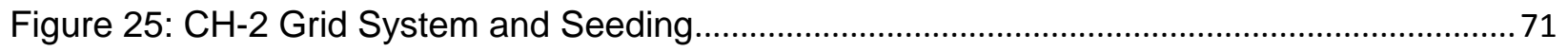

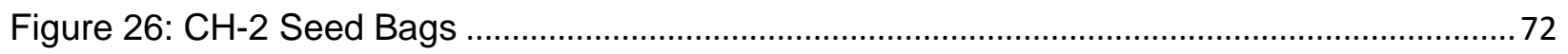

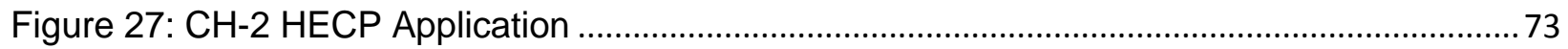

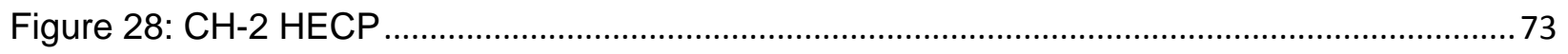

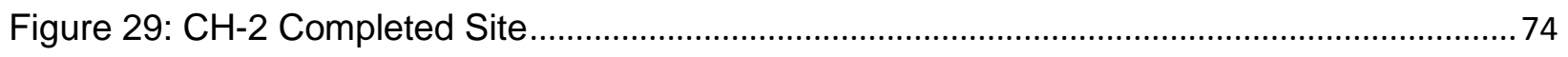

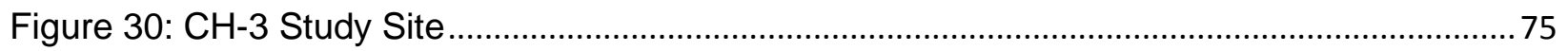

Figure 31: $\mathrm{CH}-3$ Subplot Layout $(\mathrm{TS}=$ topsoil, NTS= no topsoil, HP= hydraulic erosion control

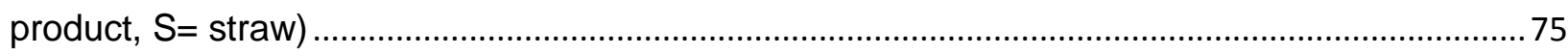

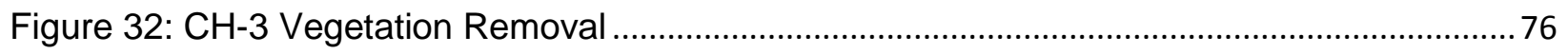

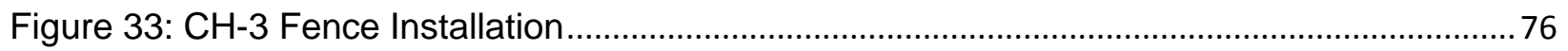

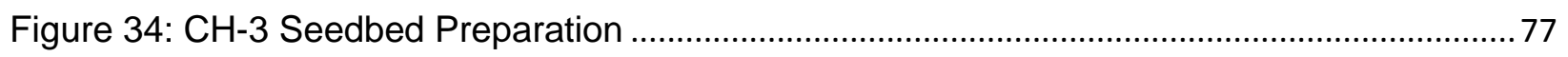

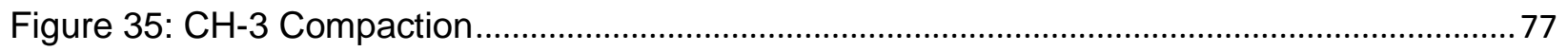

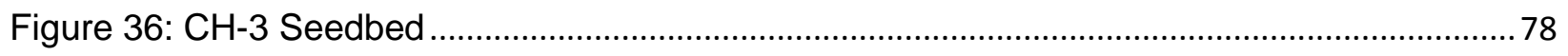

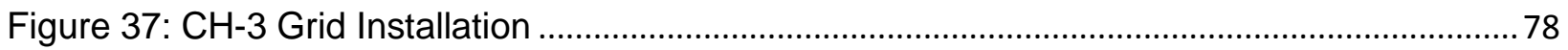




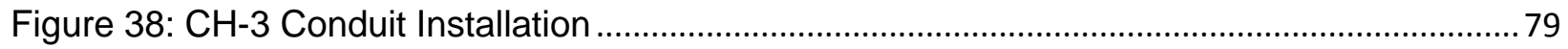

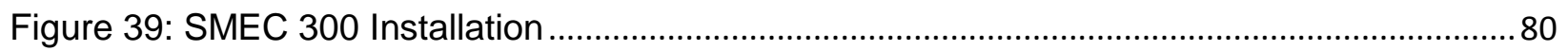

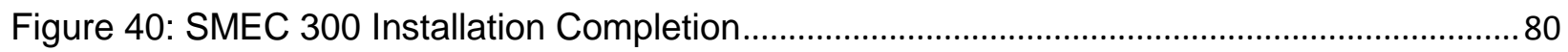

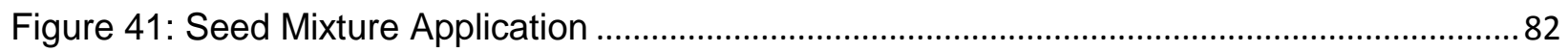

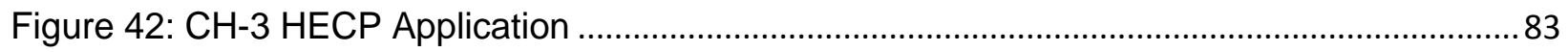

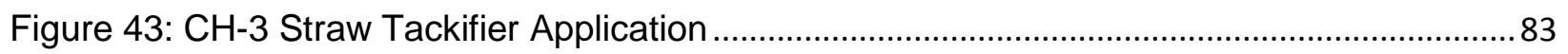

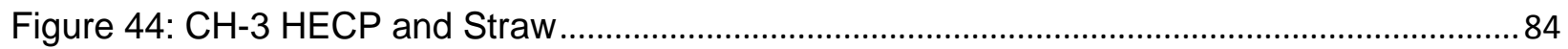

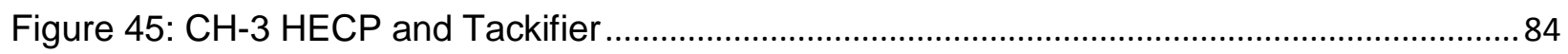

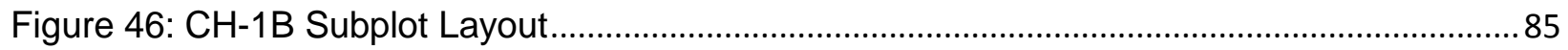

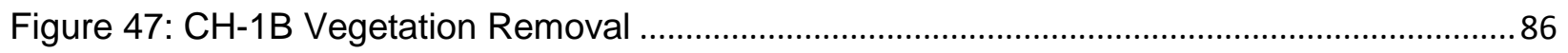

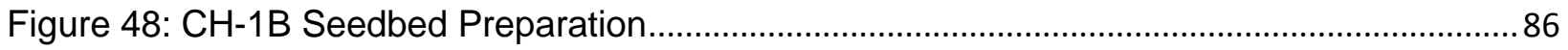

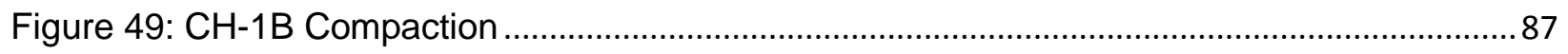

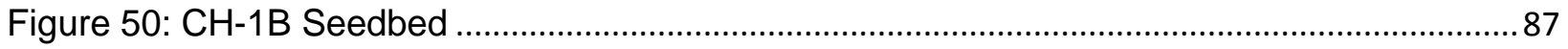

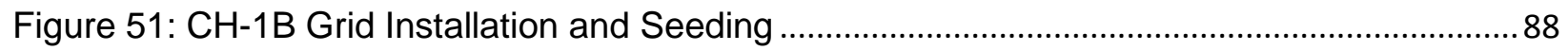

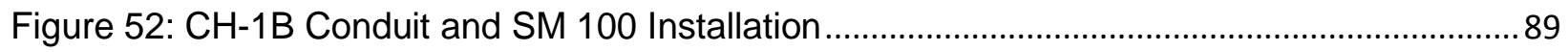

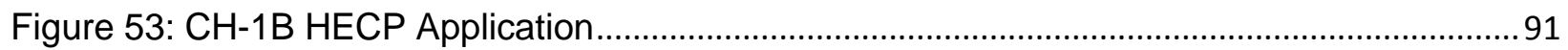

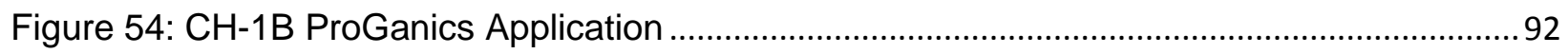

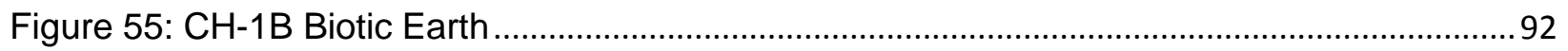

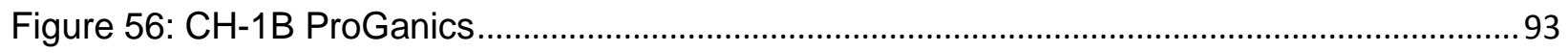

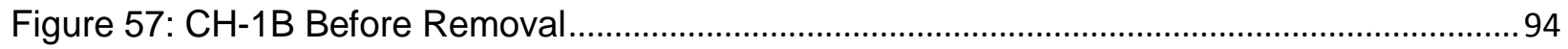

Figure 58: CH-1B After Removal ...................................................................................... 94

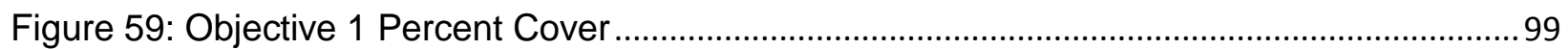

Figure 60: Objective 1 Percent Cover by Species at 51 Days ....................................................... 100

Figure 61: Objective 1 Percent Cover by Species at 90 Days ...................................................... 100

Figure 62: Objective 1 Eight Days One-way ANOVA Graph; $n=3 ; 25 \%-75 \%$ (box); median (50\%

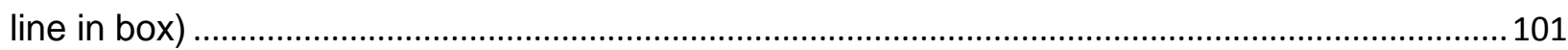

Figure 63: Objective 1 Ninety Days One-way ANOVA Graph; min and max (whisker); 25\%-75\%

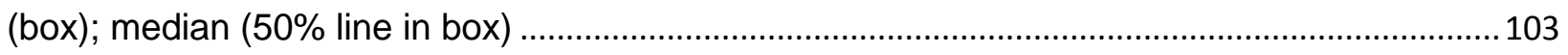

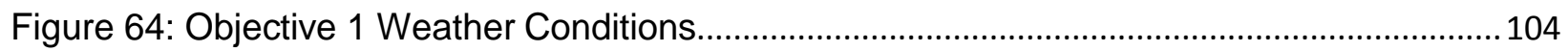

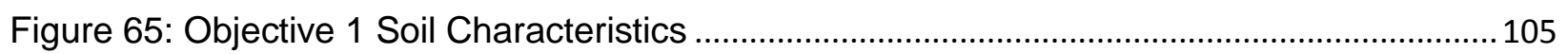

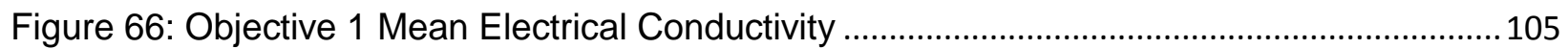

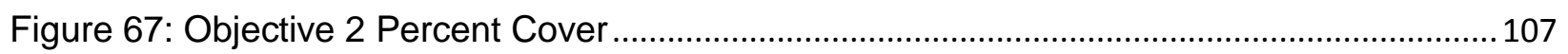

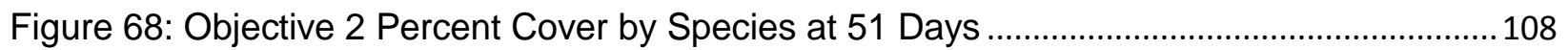

Figure 69: Objective 2 Percent Cover by Species at 90 Days ...................................................... 109

Figure 70: Objective 2 Eight Day One-way ANOVA Graph; n=3; 25\%-75\% (box); median (50\%

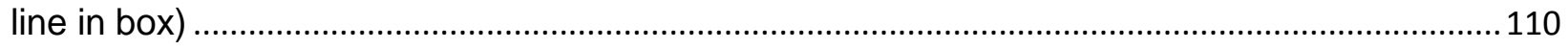

Figure 71: Objective 2 Ninety Day One-way ANOVA Graph; min and max (whisker); 25\%-75\%

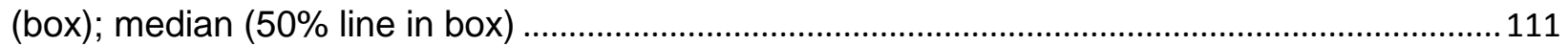

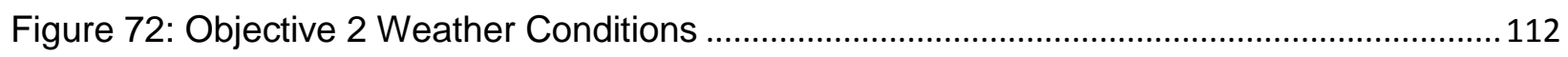

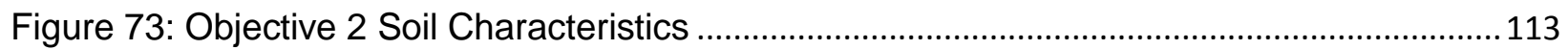

Figure 74: Objective 2 Mean Electrical Conductivity ………………………………………........ 114 
Figure 75: Objective 3 Percent Cover (TS= topsoil, NTS, no topsoil, HP= hydraulic erosion control product, $\mathrm{S}=$ straw)

Figure 76: Objective 3 Percent by Species at 51 Days (TS= topsoil, NTS, no topsoil, HP= hydraulic erosion control product, $\mathrm{S}=$ straw)

Figure 77: Objective 3 Percent by Species at 90 Days (TS= topsoil, NTS, no topsoil, HP= hydraulic erosion control product, $\mathrm{S}=$ straw).

Figure 78: Objective 3 Eight Day One-way Blocked ANOVA of Percent Cover by Seed Mixture Graph; min and max (whisker); 25\%-75\% (box); median (50\% line in box) 118

Figure 79: Objective 3 Eight Day One-way Blocked ANOVA of Percent Cover by Treatment Graph; min and max (whisker); 25\%-75\% (box); median (50\% line in box); (TS= topsoil, NTS, no topsoil, $\mathrm{HP}=$ hydraulic erosion control product, $\mathrm{S}=$ straw) 119

Figure 80: Objective 3 Ninety Day One-way Blocked ANOVA of Percent Cover by Seed Mixture Graph; min and max (whisker); 25\%-75\% (box); median (50\% line in box) 121 Figure 81: Objective 3 Ninety Day One-way Blocked ANOVA of Percent Cover by Seed Mixture Graph; min and max (whisker); 25\%-75\% (box); median (50\% line in box) 121

Figure 82: Objective 3 Weather Conditions 122

Figure 83: Objective 3 Mean Volumetric Water Content............................................................... 123

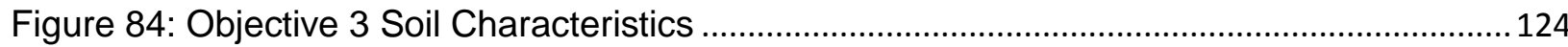

Figure 85: Objective 4 Percent Cover .............................................................................................. 126

Figure 86: Objective 4 Percent Cover by Species at 51 Days ................................................. 127

Figure 87: Objective 4 Percent Cover by Species at 90 Days ....................................................127

Figure 88: Objective 4 Eight Day One-way ANOVA Graph; n=3; 25\%-75\% (box); median (50\%

line in box) 128

Figure 89: Objective 4 Ninety Day One-way ANOVA Graph; min and max (whisker); 25\%-75\% (box); median (50\% line in box) 129

Figure 90: Objective 4 Mean Volumetric Water Content. 130 


\section{List of Tables}

Table 1: Developed Type A- Mowable Areas Seeding Rates ........................................................26

Table 2: Developed Type B- Warm Season Seeding Rates ............................................................... 27

Table 3: Developed Type C- Cool Season Seeding Rates .............................................................28

Table 4: Developed Type D1- High Elevation Seeding Rates ........................................................29

Table 5: Developed Type D2- High Elevation Seeding Rates ............................................................30

Table 6: Developed Type E- Wet Areas Seeding Rates............................................................... 31

Table 7: Developed Type A-Mowable Areas Seed Mixture; Planting time periods: Spring (March

1st - June 20th) and (August 1st - October 31st) for Summer and Fall........................................ 34

Table 8: Mowable Areas Species Occurrence .................................................................................. 34

Table 9: Developed Type B-Warm Season; Planting time periods: Spring (March 1st - June

20th) and Fall (October 1st - November 1st) ............................................................................. 37

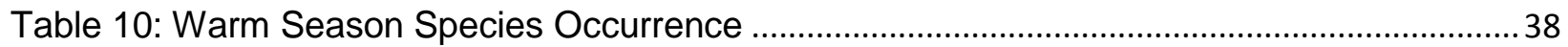

Table 11: Developed Type C-Cool Season Seed Mixture; Planting time periods: Summer and

Fall (August 1st - October 31st); Use an erosion control Birdsfoot Trefoil strand ........................ 40

Table 12: Cool Season Species Occurrence .................................................................................... 40

Table 13: Developed Type D1-High Elevation Seed Mixture; Planting time periods: Spring

(March 1st - June 20th) and Fall (October 1st - November 1st); For Slopes $\leq 3: 1(\mathrm{H}: \mathrm{V})$... 44

Table 14: Developed Type D2-High Elevation Seed Mixture; Planting time periods: Spring (March 1st - June 20th) and Fall (October 1st - November 1st); For Slopes > 3:1 ...................... 45

Table 15: High Elevation Species Occurrence ............................................................................... 46

Table 16: Developed Type E-Wet Areas Seed Mixture; Planting time periods: Spring (March

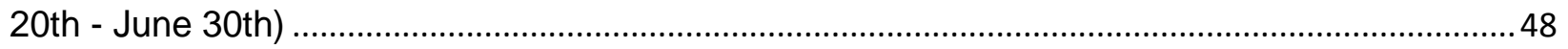

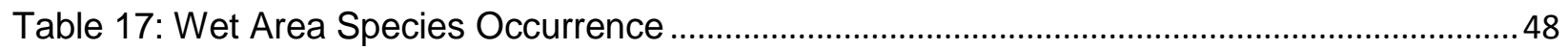

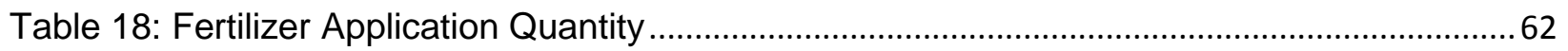

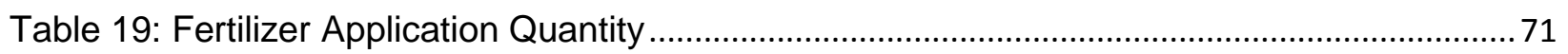

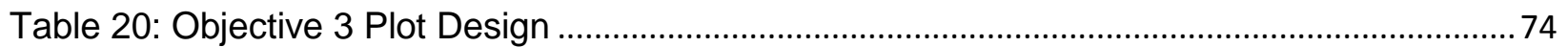

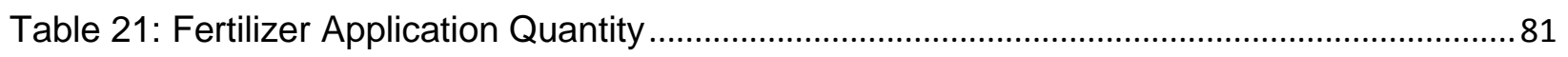

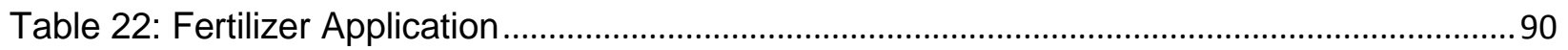

Table 23: Objective 1 Eight Days One-way ANOVA Statistics ................................................... 101

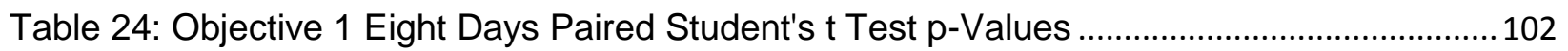

Table 25: Objective 1 Eight Days Connecting Letters Report....................................................... 102

Table 26: Objective 1 Ninety Days One-way ANOVA Statistics ......................................................103

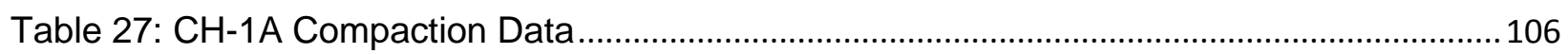

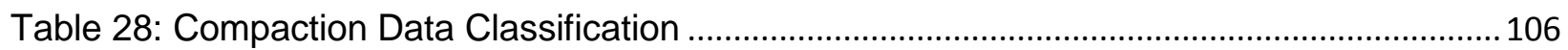

Table 29: Objective 2 Eight Day One-way ANOVA Statistics .......................................................... 109

Table 30: Objective 2 Eight Day Paired Student's t Test p-Values .............................................110

Table 31: Objective 2 Eight Day Connecting Letters Report........................................................... 111

Table 32: Objective 2 Ninety Day One-way ANOVA Statistics ...................................................... 111

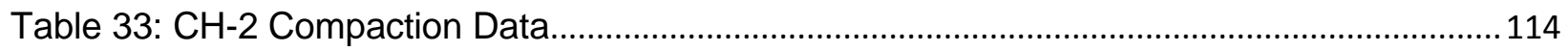


Table 34: Objective 3 Eight Day One-way Blocked ANOVA of Percent Cover by Seed Mixture Statistics

Table 35: Objective 3 Paired Student's t Test $p$-Values for Percent Cover by Seed Mixture Analysis 118

Table 36: Objective 3 Connecting Letters Report for Percent Cover by Seed Mixture Analysis118 Table 37: Objective 3 Eight Day One-way Blocked ANOVA of Percent Cover by Treatment Statistics

Table 38: Objective 3 Paired Student's t Test p-Values for Percent Cover by Treatment Analysis

Table 39: Objective 3 Connecting Letters Report for Percent Cover by Treatment Analysis .... 120

Table 40: Objective 3 Ninety Day One-way ANOVA Statistics ..................................................... 120

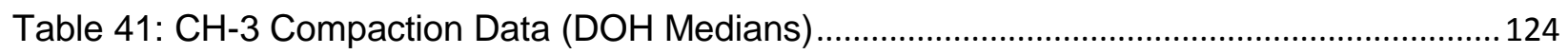

Table 42: CH-3 Compaction Data (Warm Season) ....................................................................... 125

Table 43: CH-3 Compaction Data (Cool Season) ....................................................................... 125

Table 44: Objective 4 Eight Day One-way ANOVA Statistics .................................................... 128

Table 45: Objective 4 Ninety Day One-way ANOVA Statistics .................................................... 129

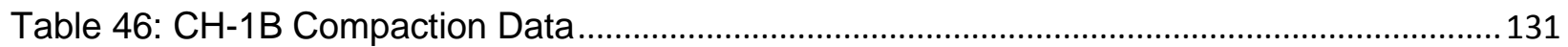

Table 47: Objective 1 Percent Cover Over 90 Days ( $n=3$ for each treatment) ........................... 144

Table 48: Objective 1 Percent by Species Data at 51 Days ( $n=3$ for each treatment)............... 144

Table 49: Objective 1 Percent by Species Data at 90 Days ( $n=3$ for each treatment)............... 144

Table 50: Objective 2 Percent Cover Data Over 90 Days ( $n=3$ for each treatment) .................. 145

Table 51: Objective 2 Percent by Species Data at 51 Days ( $n=3$ for each treatment)............... 145

Table 52: Objective 2 Percent by Species Data at 90 days ( $n=3$ for each treatment) ................ 145

Table 53: Objective 3 Percent Cover Over 90 Days ( $n=2$ for each treatment)........................... 146

Table 54: Objective 3 Percent by Species Data at 51 Days ( $n=2$ for each treatment) ............... 147

Table 55: Objective 3 Percent by Species Data at 90 Days ( $n=2$ for each treatment) ............... 147

Table 56: Objective 4 Percent Cover Over 90 Days ( $n=3$ for each treatment) ............................. 148

Table 57: Objective 4 Percent by Species Data at 51 Days ( $n=3$ for each treatment)............... 148

Table 58: Objective 4 Percent by Species Data at 90 Days ( $n=3$ for each treatment)............... 148 


\subsection{Introduction}

\subsection{Background}

The research project was tasked by the West Virginia Division of Highways (WVDOH) to examine and recommend alterations to their seeding and mulching specifications. The current seed mixtures and mulch specifications were evaluated to determine potential areas of improvement that could be tested in the field. The goal was to determine and test various reclamation techniques to improve WVDOH construction reclamation success. Seedbed preparation, soil amendment and media products, mulch products, and alternative seed mixtures were the primary focus to improve re-vegetation processes in West Virginia.

The objective for the developed seed mixtures was to use native or low invasive species. Desirable erosion control species were selected and then compared to the West Virginia Division of Natural Resources (WVDNR) Natural Heritage Program (2009) list of published invasive species. Threat level 1 and 2 species were avoided, where threat level 3 species were deemed acceptable for erosion control use. The developed mixtures were then evaluated in the field to examine percent cover. The goal was to establish and maintain a minimum of $70 \%$ cover in the first growing season. The $70 \%$ minimum cover value was chosen based on National Pollution Discharge Elimination (NPDES) permit requirements.

Following field testing of the developed seed mixtures and seedbed preparations, recommendations will be made to the WVDOH "Section 652-Seeding and Mulching" specification (WVDOH 2010). The recommendations will then be used to aid in improving the current specification. 


\subsection{Objectives}

This research evaluated if seed mixtures that include native species could be applied and addressed two main objectives:

1. Recommend alternative seeding mixtures that include native species.

2. Test vegetation establishment and persistence of the recommended species.

\subsection{Study Design}

Objective 1 was completed by developing alternative seeding mixtures that included native species. The process began by determining which native and non-native species occur and are present in West Virginia. The species were then compared based on species characteristics, economics, and erosion control use. After initial species selection, specialists were consulted and recommended species and seeding rates based on their industry experience and knowledge. A total of six mixtures were created.

Objective 2 was tested through a field study. Four specific objectives were considered:

1. Compare the proposed seed mixtures to the current standard.

2. Evaluate the need for a high elevation seed mixture.

3. Determine if seed bed preparation influences proposed seed germination and cover.

4. Evaluate the performance of soil amendments in comparison to topsoil and no topsoil.

In the following sections, the field methods are divided into study design and a plot study was completed. Over the course of the study, data were collected for topography, vegetation cover and biomass, precipitation, temperature, and soil characteristics. 


\subsection{Background}

\subsection{Seed Mixture Design}

Proper seed and seeding methods are crucial to any reclamation project, especially in controlling erosion. With the ongoing controversy of invasive species, a seed mix should focus on native species to reduce the introduction and spread of invasive species. Tyser et al. (1998) recommends that seed mixtures should predominantly be native grasses. Li et al. (2008) recommends the use of native species because they are adapted to the location's natural environment, but they may be uneconomical because of the lack of native seed production. Seed selection is unique to each site and should be taken seriously. Large sites, wildlife habitat enhancement, conservation, and beautification projects need their own seed mixtures determined because standard mixtures are inadequate (MacDonagh and Hallyn 2010).

Some invasive species are using the United States highway system to spread and overtake the native species. Rentch et al. (2005) suggests that highway agencies need to develop methods to watch and control non-native species and promote native species. Li et al. (2008) examined successional processes, erosion control traits of the vegetation, and mowing implications along roadways. The study found that mowed areas in Texas had higher amounts of non-native grass species adapted to mowing but that the mixtures planted by the Texas Department of Transportation did not show any species to be invasive in nature. Areas not mowed had a reduction in the adapted non-native species and a population increase of native species (Li et al. 2008). Highways should be considered ongoing biological projects instead of short project timelines, and the effects of construction on the spread of non-native species should be considered significant (Tyser et al. 1998).

Steinfeld et al. (2007a) recommends creating a comprehensive list of species, but this could be limited to a project's scope to decrease background research hours. For example, this study is focusing on grasses, legumes, and forbs. The next step would be to narrow the list to species that best fit the project's characteristics. The focus will be on finding primary species, 
species groups that work well together, and possibly experimental species. Primary plant species are species that occur in several environments, are readily available in nature, perform well by roads, and reproduce well. The limiting process will be based on characteristics of the plant species and whether they will be able to perform proficiently for the project.

When creating a seed mixture or examining a commercial mix, there are a variety of species to consider, including grasses, forbs, woody plants, and nurse crops (Salon and Miller 2012). Sanderson et al. (2012) reported that mixtures consisting of grasses and forbs were more effective in reducing weeds than monocultures and that selecting appropriate seed species is more imperative than uniformity of species in a mix. MacDonagh and Hallyn (2010) state that for large projects of 10 or more acres, a site specific mix should be used. When selecting seed, one should choose species that meet the project's requirements, such as erosion control or wildlife habitat (Houck 2009). Sterile cover crops can be used to establish a quick cover and reduce erosion, and they will shelter the perennial species for a year (MacDonagh and Hallyn 2010 and Bureau of Land Management, Farmington Field Office 2013).

Mixtures designed to incorporate succession of seed species are important because roadside soil conditions change over time and the vegetative cover will need to change with it (California Department of Transportation 2014). An example would be the ability of annual species to grow on bare and nutrient poor soils which will help promote overtake of perennial species. West Virginia Division of Highways experiences secondary succession with their projects. Secondary succession occurs on sites that had previous vegetation removed by natural or human events like construction or hurricanes. An abandoned site with bare soil has a succession process that begins with annual species proceeding to biennial and grass species the following year. After 3 to 4 years, herb and shrub perennials take over, leading to softwood tree species 5 to 15 years later. Finally, hardwood tree species take over around 50 to 75 years later (Pidwirny 2006). Therefore, a mixture designed with succession in mind can promote the vegetative process and potentially reduce revegetation problems. 
A study done for the Minnesota Department of Transportation recommends determining the ecological regions within a state so that correct plant species and communities can be developed for each ecological region. A list of commercially available grass and forb species should then be compiled. Finally, one should choose a cover crop and determine the application rate. After conducting research and literature review, develop seed mixtures that best suit each zone. (MacDonagh and Hallyn 2010)

When developing a list of seed species, Hogan and Drake (2009a) suggest asking the following questions:

- $\quad$ Are they native species?

- Are the non-native species invasive or could they become a problem in the future?

- Are the right plant species being used in the mixture design?

- "Is the plant species easy to establish?"

- Are the plant species fast germinators?

- $\quad$ Are the plant species available for purchase locally?

- Are the plant species economical for projects?

- Will the species be adequate for erosion control?

- Are the plant species the right fit for the project?

- Do the plant species require additional water?

Through various studies and government agency guides, numerous suggestions have been made to aid in seed and species selection. These recommendations should assist with the thought and examination process of creating seed mixture designs. The following list details their recommendations and concerns:

- Determine the characteristics of the site's soil and topography to aid in species selection (Dickerson, et al. 1998). 
- Select the correct seed species because it is important to the restoration process (Bochet et al. 2010).

- Select dominant species that occur in a large range of ecological environments (Steinfeld et al. 2007a).

- Avoid using only one species in a mixture (Steinfeld et al. 2007b). A larger number of plant species in a seed mixture assists in reducing invasive species (Oakley and Knox 2013).

- Use a variety of species to colonize an assortment of microsites that are typical of a highly disturbed site (Steinfeld et al. 2007b).

- Use a native or non-native mixture; do not mix the two types (Houck 2009).

- Design a mixture that has good succession between species (California Department of Transportation 2014).

- A seed mixture should contain a small amount of annuals with perennial grasses and forbs (California Department of Transportation 2014).

- Use 6-10 grass species and 15-20 herbaceous species (Kirmer et al. 2012).

- Incorporating legumes in a mixture is important for their nitrogen fixation abilities (Salon and Miller 2012).

- Reubens et al. (2007) focused on the effects of roots on shallow slope stability and erosion control and found that a combination of fine roots and coarse roots was advantageous.

- Examine the quality of seed (Salon and Miller 2012).

- Purchase seed by pure live seed (PLS) (Salon and Miller 2012).

- Inquire from retailors what the best seed lots are (Salon and Miller 2012).

- Obtain seed quotes from at least 3 suppliers (Dickerson, et al. 1998).

- Obtain the seed analysis report for seeds (Dickerson, et al. 1998). 
- Choose seed from lots with excellent germination rates (Salon and Miller 2012).

- Set a standard for zero tolerance of weed species in seed (Dickerson, et al. 1998).

- Purchase unmixed seed and mix according to the project preference (Dickerson, et al. 1998).

- A sterile cover crop can be used for initial soil stabilization (Bureau of Land Management, Farmington Field Office 2013).

- There is a need to examine seed mixtures that germinate quickly for erosion control measures (Storey et al. 2011).

- Plant cool season mixtures from August 15 to September 1 (Salon and Miller 2012).

- Plant warm season mixtures in the spring (Salon and Miller 2012).

- Experiencing bad results from 10-15 PLS rate for a warm season mix typically means that an error occurred with the method or project characteristics (Dickerson, et al. 1998). These suggestions ensure that the correct plant species are chosen for the ecological, climatic, and soil requirements of site. The developed species list will then allow easier selection of seed mixtures with adequate species quality and characteristics. Roberts (2001) gives a description for each species based on its general information, fertilization requirements, maintenance level, sun requirements, mowing preferences, water preferences, variety of species, and soil requirements.

Once the seed mixture species have been determined, the next step is to determine the correct seeding rates. Storey et al. (2011) suggests an application rate of 20-50 seeds per 0.97 $\mathrm{ft}^{2}\left(0.09 \mathrm{~m}^{2}\right)$ which should be increased for sites with rocky terrain, deficient soil, or conditions that may result in seed loss. However, the California Department of Transportation (2014) states that a seeding rate of $80-100$ seeds per $0.97 \mathrm{ft}^{2}\left(0.09 \mathrm{~m}^{2}\right)$ is typically used and to change the number according to the mature plant size of the specific species. Seeding rates have to be determined for each site, dependent on the site conditions, and published or recommended 
rates are guides to get to the correct required rate. The right seeding rate needs to be applied because high seeding rates can be disadvantageous to vegetative measures. This causes overcrowding, which reduces the number of seedlings. Low seeding rates can be insufficient (Storey et al. 2011). To aid in the development process, it may be advantageous to contact an appropriate horticulturist or agronomist for guidance.

\subsection{Mulches}

The goal of any erosion control project is to control the effects of erosion in order to meet state and federal regulations and guidelines. Mulching has been shown to reduce the effects of erosion and increase the percent of vegetative cover (Dunifon et al. 2011, Storey et al. 2011). Common mulches utilized are straw, hay, wood chips or wood fibers, organic matter, compost, and hydraulic erosion control products (HECP). Mulch is an effective erosion control measure because it reduces runoff by increasing friction and therefore slowing the runoff velocity (Robichaud et al. 2013). Mulches improve infiltration rates of soils and retain moisture that can later be absorbed by the plant species during drier climatic times (Smets et al. 2008).

In a study on Terrace Mountain in Canada, wood and straw mulch treatments reduced sediment yield and runoff velocity compared to the control plot, indicating that the mulch was more effective at reducing erosion and retaining the soil on the slope surfaces compared to bare soil (Robichaud et al. 2013). With the addition of mulch, site soil conditions experience an increase in water content due to an increased infiltration rate which can drastically improve growth rate and health of a vegetative cover (Smets et al. 2008). Mulch has been found to work more effectively on longer slopes compared to any geotextile products for soil loss ratio in rill and interrill erosion. However, geotextiles are more effective in reducing soil loss ratios in rill and interrill erosion on shorter plots (Smets et al. 2008). Natural geotextile products are claimed to be better for erosion control than synthetic products (Bhattacharyya et al. 2010).

When deciding on what type of mulch to utilize, a list of the characteristics of each mulch should be examined to make sure the project's goal is met. Various mulches may not be 
economical to purchase or could contain unfavorable traits based on the location of the project. Salon and Miller (2012) have created a table to assist in mulch selection and application. Straw mulch is a good example because straw is both economical and easy to spread, but it may contain undesired seed species. Raw straw costs between $\$ 0.02$ and $\$ 0.04$ per $9.04 \mathrm{ft}^{2}(0.84$ $\mathrm{m}^{2}$ ). Straw also has a short life span as a mulch, typically lasting two years, and it can be redistributed by winds (Robichaud et al. 2013). A tackifier can be used to retain straw mulch to the application site (Salon and Miller 2012, Landis et al. 2005). Benik et al. (2003) observed that straw mulch had the highest vegetative growth compared to their studies of other products. Hay mulch is similar to the traits of straw mulch and increased the survival rate of E. nigrum and J. communis cuttings in a Mallik and Karim (2008) study. Coarser mulches like wood mulch are recommended for sites with soil susceptible to erosion (Bakr et al. 2012). Wood mulches last approximately four years. If the location requires the removal of several woody species, the use of wood mulch may be economical, because the resources will already exist on location, but transporting wood mulch to large project sites may be unfeasible depending on budget restraints (Robichaud et al. 2013). Organic matter mulch in the Mallik and Karim (2008) study was comprised of topsoil collected along the roadside construction and applied at a thickness of 0.10-0.16 ft. (3-5 cm). A soil with higher levels of organic matter has an improved vegetative growth and experiences a larger variety of species (Skrindo and Halvorsen 2008). Dunifon et al. (2011) noted that when organic matter was added to the surface of a compacted soil, common in construction sites, it stayed at the surface. This could be due to the creation of a barrier that is difficult to breach.

Compost mulch can include any material from yard waste to poultry litter that has been composted and is ready for application. Hansen et al. (2012) experienced positive results from a compost mixture of equal parts compost and woodchips but concluded that further research is required to examine the long term water quality effect. Compost improves soil properties and will increase vegetative growth (Dunifon et al. 2011). In a study by Storey et al. (2011), the 
researchers did not discover a definite difference in vegetative cover between the seed mixtures, but they did find a difference between bare and composted treatments. Storey et al. (2011) found that composted plots achieved $70 \%$ vegetative coverage within nine months, outperforming non-composted plots by over 10 months. The compost was comprised of $75 \%$ topsoil and $25 \%$ manure compost applied at a depth of 0.025 meters and disked to a depth of $0.33 \mathrm{ft} .(0.10 \mathrm{~m})$. The study concluded that the increased vegetation was likely due to the compost improving the soil structure, nutrient concentrations, organic matter, and water holding capacity and decreasing evaporation compared to non-composted treatments (Storey et al. 2011).

Faucette et al. (2006) experimented with a variety of compost blankets to compare against hydroseeding results and found that the compost treatments had a higher vegetative cover in the first three months, lower weed biomass, improved soil quality after 18 months, and less phosphorus negatively affecting water runoff. Faucette et al. (2006) followed the American Association of State Highway Transportation Officials (AASHTO) specifications for compost application at a depth of $0.03 \mathrm{ft} .(3.75 \mathrm{~cm})$.

Bakr et al. (2012) recommends that a mulch and compost mixture should be used on construction sites and existing locations. They also state that tilling a compost and mulch mixture into the soil is not a good method because it increases erosion (Bakr et al. 2012). Smets et al. (2008) says that soil texture, type of mulch, and slope have an effect on a mulch's ability to control erosion, and one can look at this conclusion as a recommendation to examine the impact of these characteristics on the effectiveness of mulch.

Hydromulching is an effective means of application in areas that are hard to access. Hydromulch can be mixed onsite with mulch, pellet, wood fiber, seed, fertilizer, lime, tackifier, and various other additives to meet site requirements (Tyser et al. 1998, Landis et al. 2005). Landis et al. (2005) found that the tackifier can degrade and break apart under severe cold, 
leaving the slope at risk for erosion. However, it performed well in controlling erosion on another site with steep slopes.

Since revegetation and achieving regulations for percent vegetative cover can be difficult, evaluating project sites and picking appropriate erosion control measures can assist in revegetation efforts. A bare soil is not the most effective modern practice, so a mulching measure can be chosen to help improve soil properties, moisture content, runoff rate, and sediment yield to aid in faster vegetative growth and reduced damage from erosion. Proper site evaluation, testing, and planning can lead to a successful revegetation project.

\subsection{Seed Bed Preparation}

In conjunction with choosing appropriate seed mixtures and mulches, seed bed preparation is imperative for the success of vegetation establishment. The Bureau of Land Management Farmington Field Office (2013) advises that seedbed preparation is extremely important for re-vegetative success. Landis et al. (2005) advises that each site should be examined based on soil and climate so that the proper revegetation practices can be conducted. For road construction projects, the smallest surface area possible should be disturbed to limit the effects (Tyser et al. 1998).

Seed bed preparation begins with the removal and stock piling of topsoil. During the removal process, vegetative debris and rock need to be removed from the topsoil to allow for appropriate grading later in the seed bed preparation process. Based on the project construction timeframe, the exposed topsoil stockpile may need a temporary cover crop applied to control the effects of erosion and reduce the loss of soil from the stockpile. Topsoil should not be applied to slopes greater than 2:1 because erosion and sedimentation of the topsoil becomes a problem (Salon and Miller 2012). Once the earth work of a project is completed, preparation of the seed bed can begin.

The next steps of seed bed preparation are to determine if the subsoil is over-compacted and to perform soil analysis on the subsoil and topsoil. Compaction can be determined based 
on a bulk density examination. If subsoil is not over-compacted, soil fracturing is not necessary to perform. However, if over-compaction has been detected, then the subsoil will need to be scarified before topsoil is applied to the site. Sandy clays, silty clays, clays, and some clay loams have an ideal bulk density of $<68.7 \mathrm{lb} / \mathrm{ft}^{3}\left(<1.10 \mathrm{~g} / \mathrm{cm}^{3}\right)$ and a restrictive bulk density of $>91.8-98.6 \mathrm{lb} / \mathrm{ft}^{3}\left(>1.47-1.58 \mathrm{~g} / \mathrm{cm}^{3}\right)$. Silts and silt loams have an ideal bulk density of $<81.2 \mathrm{lb} / \mathrm{ft}^{3}$ $\left(<1.30 \mathrm{~g} / \mathrm{cm}^{3}\right)$ and a restrictive bulk density of $>109.2 \mathrm{lb} / \mathrm{tt}^{3}\left(>1.75 \mathrm{~g} / \mathrm{cm}^{3}\right)$. Sandy loam, loams, sandy clay loams, clay loams, silt loams, and silty clay loams have an ideal bulk density of $<87.4 \mathrm{lb} / \mathrm{ft}^{3}\left(<1.40 \mathrm{~g} / \mathrm{cm}^{3}\right)$ and a restrictive bulk density of $>103.0-112.4 \mathrm{lb} / \mathrm{ft}^{3}\left(>1.65-1.80 \mathrm{~g} / \mathrm{cm}^{3}\right)$. Sands and loamy sands have an ideal bulk density of $<99.9 \mathrm{lb} / \mathrm{ft}^{3}\left(<1.60 \mathrm{~g} / \mathrm{cm}^{3}\right)$ and a restrictive bulk density of $>112.4 \mathrm{lb} / \mathrm{ft}^{3}\left(>1.80 \mathrm{~g} / \mathrm{cm}^{3}\right)$ (Salon and Miller 2012). The ideal and restrictive bulk densities will aid in compaction analysis. Over-compacted subsoil on slopes less than 3:1 should be scarified to a depth no less than 0.167-0.102 ft (0.051-0.102 m). For subsoil on slopes from 3:1 to 2:1, the subsoil should be scarified to a depth of $0.042-0.082 \mathrm{ft}(0.013-0.025$ m). The scarification decreases the restrictive nature of the soil to the vegetation root growth (Salon and Miller 2012). Hogan and Drake (2009b) emphasized that soil should be loose before seeding to ensure proper root growth.

The topsoil should be analyzed to determine the $\mathrm{pH}$, organic matter content, soluble salts, and nitrogen, phosphorus, and potassium levels (N-P-K). Soil amendments like fertilizer and lime should only be used if crucial to the development and long-term health of the sown seed species. Soil amendments are determined crucial if a soil test determines that the sample failed to meet the nutritional requirements of the seed species. Soil should be examined before applying a treatment as it may not need supplementation to meet the specific requirements. Skrindo and Halvorsen (2008) commented that soils with proper nutrients - $\mathrm{pH}$ 6.0-7.0 and appropriate amounts of phosphorus, nitrogen, and potassium (Salon and Miller 2012) - do not need fertilization for natural revegetation. Salon and Miller (2012) state that a soil should be tested for $\mathrm{pH}$, percent fines for sandy or gravely soils, bulk density for compacted soils, and soil 
nutrients so that adjustments can be made for lime, macronutrients, and micronutrients. The Bureau of Land Management Farmington Field Office (2013) also recommends that the soil be tested in order to develop a reclamation plan for revegetation. Hargett et al. (1982) recommends each site to have a soil analysis performed to determine what measures need to be taken to achieve sufficient vegetative growth to limit the effects of erosion. Standard fertilizer and lime applications may be inadequate for severe cases or could be overestimated, causing contamination in runoff water and leading to negative environmental effects. For topsoil, the $\mathrm{pH}$ should be between $5.5-7.5$, organic matter no less than $2 \%$ by weight, soluble salts less than 0.5-1.0 dS/m, and appropriate N-P-K concentrations. Subsoil should be analyzed for $\mathrm{pH}$ and should be greater than 4.0. If the soil is acidic, lime should be added to the subsoil to correct the $\mathrm{pH}$ level (Salon and Miller 2012).

Immediately after the compaction and soil analysis have been performed and the appropriate measures have been made to remediate undesirable characteristics of the subsoil, the site can be prepared for topsoil application. To aid in topsoil stability, the subsoil should be tracked with a bulldozer to create check slots to interlock the topsoil with the subsoil. Once the slope has been tracked and the soil and site are dry and workable, the topsoil should be applied at a depth of $0.417-0.666 \mathrm{ft}(0.127-0.203 \mathrm{~m})$ and tracked to no less than $0.335 \mathrm{ft}(0.102 \mathrm{~m})$. After the topsoil has been applied and compacted by tracking, a final scarification of $0.249-0.499 \mathrm{ft}$ $(0.076-0.152 \mathrm{~m})$ of the topsoil is necessary for slopes less than 3:1. The seedbed should be firm and friable with no large stones or soil clods. Immediately after preparing the surface, apply desired seed, mulch, lime and fertilizer. Fertilizer and lime can be applied during the scarification process for slopes less than 3:1 (Salon and Miller 2012). When applying seed, the seed and soil should be mixed and packed to ensure that the seeds are in adequate contact with the soil to prevent them from being washed away or eaten (Hansen et al. 2012, Sanderson et al. 2012). 
For slopes from $3: 1$ to $2: 1$, scarify the soil to a depth of no less than 1 inch, but track the slope to aid in erosion control and the establishment of microsites. Again, immediately apply desired seed, mulch, lime, and fertilizer for the site. Fertilizer and lime can be added during the scarification process. Use the proper seeding method based on project characteristics (Salon and Miller 2012).

Once a project is seeded and mulched, the project is still not necessarily complete. To ensure project success, post examination and care will most likely be required. One should visit the project site periodically to check the vegetative growth and determine if the project needs additional seeding, fertilizer, or lime for healthy vegetative development (Hogan and Drake 2009b). Some project sites may require multiple additional treatments to get the desired results outlined in the project goals. Benik et al. (2003) and WVDEP (2006) recommended that if an erosion control measure failed, the area should be reseeded to try and reestablish desired vegetative cover immediately. 


\subsection{Developing Seed Mixtures}

\subsection{Introduction}

The West Virginia Division of Highways (WVDOH) presently conducts projects based on the "Standard Specifications Road and Bridge" that was adopted in 2010 (WVDOH 2010). Section 652 "Seeding and mulching" outlines the approved methods, application, and materials used for seeding and mulching (WVDOH 2010). The purpose of this work was to review and update the specifications. This research focused on developing an experimental list of seed mixtures that provide erosion control while meeting objectives of low invasiveness or native species.

Six alternative seed mixtures were proposed. All seed mixtures and species were selected to provide slopes with erosion control. Developed Type A seed mixture was intended to be planted close to roadways with the characteristic of being low-growing to reduce mowing occurrence and provide a clear site distance. The mixture is a turf mixture of low invasive nature for WVDOH facilities, rest areas, and mowable areas. Developed Type B seed mixture was intended for cut and fills slopes outside of mowing areas and has tall growth characteristics for spring planting. Developed Type C was designed to provide a mixture for late summer and early fall planting that was of low invasive nature for cut and fills. Developed Type D1 and Type D2 mixtures were developed for elevations higher than 2,400 $\mathrm{ft}(731.52 \mathrm{~m})$ to meet the need requested by the WVDOH. Native species were chosen because they naturally inhabit high elevations in West Virginia. Developed Type E mixture was developed for wet areas where normal vegetation cannot be established due to over saturated conditions.

\subsection{Methods}

\subsubsection{Seed Mixture Types}

A total of six seed mixtures were developed based on the evaluation of current mixtures, WVDOH requests, and WVDNR recommendations. The seed mixtures are as follows: 
- Developed Type A: Mowable Areas

- Developed Type B: Warm Season

- Developed Type C: Cool Season

- Developed Type D1: High Elevation (>2,400 ft, $731.52 \mathrm{~m})(\leq 3: 1$ Horizontal:Vertical $(\mathrm{H}: \mathrm{V}))$

- Developed Type D2: High Elevation (>2,400 ft, $731.52 \mathrm{~m})(>3: 1 \mathrm{H}: \mathrm{V})$

- Developed Type E: Wet Areas

Each mixture was designed to fulfill specific reclamation objectives for highways in West Virginia. Developed Type A is a mixture composed of non-native species with low growth characteristics for mowable areas. Mowable areas would include locations along roads, WVDOH facilities, and rest areas that will be maintained by mowing equipment. The non-native mixture is intended for spring, late summer, and early fall planting. The vegetated height of the mixture was determined to reach up to $3.5 \mathrm{ft}$ (1.07 m) (Ernst Conservation Seeds 2014). The low growth characteristics could allow for a decrease in required mowing occurrence. The decrease in mowing could potentially provide large savings for the WVDOH.

Developed Type B is a mixture composed of native species to inhabit cut and fill slopes. Cut and fill slopes tend to be greater than 3:1 $(\mathrm{H}: \mathrm{V})$. The native mixture is intended for spring planting and to provide a positive ecological impact by avoiding the use of non-native species. The mixture is composed of species with larger foliage and root growth to interlock the slopes and prevent erosion.

Developed Type C was developed for cool season plantings by using minimally invasive non-native species. Native species could not be used because they do not have a short enough germination period to establish in the fall season. However, the cool season non-native species have a short germination period suitable to late summer and early fall plantings. Developed Type C replaces Developed Type B for late summer and early fall plantings only. 
Developed Type D was requested by the WVDOH because they are presently having revegetation difficulty in high elevation regions. Developed Type D utilizes native species adapted to the high elevation ecoregions in West Virginia. The mixture can be planted in the spring and the fall. Developed Type D1 is for slopes less than or equal to 3:1 $(\mathrm{H}: \mathrm{V})$ slopes and Developed Type D2 is for slopes greater than 3:1(H:V) slopes.

Developed Type E mixture was created based on the recommendation by the WVDNR, emphasizing the need of a wet area mixture. The present specification does not have a mixture to fulfill the seeding and reclamation of potential wet areas. Wet areas are not as common, but do occur and require specific adapted species. Examples of areas that may require a wet area mixture would be ditches, retention basins, detention basins, and various other drainage and storage areas along highway corridors.

\subsubsection{Species Selection}

Species selection occurred after defining the six mixture needs. The goal for each mixture was to maintain a range of $8-12$ species consisting of a nurse crop, graminoids, legumes, and forbs (Skousen and Venable 2007 and Kirmer et al. 2012). The current specification does not have any forbs in the mixtures and contains only two legumes (WVDOH 2010). Species recommendations were given by the WVDNR based on extensive knowledge and occurrence of species noted in the field by the WVDNR. A large focus was also placed on a study conducted in West Virginia by Skousen and Venable (2007) which concentrated on establishing native plant species along highways. The study recommended the addition of an annual or biennial to native mixtures being planted on new sites to act as an erosion control until the native species could establish and provide cover. The study experimented with various native species, but only recommended and documented big bluestem (Andropogon gerardii Vitman), little bluestem (Andropogon scoparius Vitman), switchgrass (Panicum virgatum L.), Indiangrass (Sorghastrum nutans [L.] Nash), partridge pea (Chamaecrista fasciculate Michx.), and brown-eyed susan (Rudbeckia triloba L.). 
During species selection, all threat level 1 and 2 species (WVDNR Natural Heritage Program 2009) were avoided due to their invasive nature. Ernst Seed's horticulturist (Mark Fiely, personal communication, January 13-29, 2015) and former Natural Heritage Vegetation Ecologist (Elizabeth A. Byers, personal communication, October $8^{\text {th }}$ - December $5^{\text {th }}$, 2014) aided in seed recommendations and selection based on carrier experience and industry knowledge. A list of criteria used for species selection included:

- Native:

- Indigenous plant species to West Virginia in accordance to USDA, NRCS (2015)

- Non-native with low invasive characteristics:

- Non-indigenous plant species to West Virginia in conjunction to USDA, NRCS (2015) and WVDNR Natural Heritage Program (2009)

- Threat Level 3 or less in accordance to WVDNR Natural Heritage Program (2009)

- Occurs in multiple ecoregions:

- Sited species occurrence based from USDA, NRCS (2015)

- Preferable occurrence of three ecoregions out of four

- Occurs in multiple elevation ranges

- Occurs in multiple precipitation ranges

- Occurs in multiple soil medias

- Occurs in two main watersheds in WV (Chesapeake Bay and Ohio River Valley)

- Occurs frequently in West Virginia:

- Comparison based on number of counties species was noted to occupy by the USDA, NRCS (2015)

- Preferred occurrence of $27 / 55-41 / 55$ counties in West Virginia according to USDA, NRCS (2015) 
- Used for erosion control:

- Noted for erosion control use by species description from USDA, NRCS (2015) and Salon and Miller (2012)

- Competitive native species pricing:

- Native species with economical pricing for one acre or greater plantings

- Low cost non-native species

- Seed available by multiple distributors

The USDA, NRCS (2015) was the primary information source used for species comparison and was used to determine suitable vegetation for each mixture type. Growth period, mature height, lifespan, soil texture, anaerobic tolerance, drought tolerance, fertility requirement, moisture use, $\mathrm{pH}$, root depth, seeding vigor, vegetative spread rate, palatability, use, and establishment traits for each species were utilized during the comparison process (USDA, NRCS 2015). The initial comparison was performed in Microsoft Excel by ranking the characteristics on a scale of $1-5$, with 1 being an undesirable trait, 3 a moderately desirable trait, and 5 a desirable trait. The scores were then totaled to indicate desirable species for the initial selection for Developed Type A-E. Desirable species were those that had the highest total ranked values.

Once desirable species were chosen, Figures 1-5 were used to verify that the selected species occupied the various environmental characteristics experienced throughout the state. The main environmental characteristics taken into consideration were ecoregions, elevations, precipitations, soils, and watersheds. USDA, NRCS (2015) species maps were used in conjunction with Figures 1-5 to determine if the desirable species inhabited multiple and in some instances all of the environmental characteristics examined in this study. 
After the species passed the environmental characteristics examination, they were finalized into mixtures and sent for review. Ernst Seed's horticulturist (Mark Fiely, personal communication, January 13-29, 2015), former Natural Heritage Vegetation Ecologist (Elizabeth A. Byers, personal communication, October $8^{\text {th }}$ - December $5^{\text {th }}, 2014$ ) and NRCS in Lexington, KY (Casey Shrader and Sonya Keith, personal communication, January 22-26, 2015) reviewed the developed mixtures for performance and compatibility. Any and all recommendations in additional or alternative species then followed the same procedures conducted for the initial species examination. After a consensus was met, the species composing the six mixtures were finalized and the seeding rates determination process begun. 


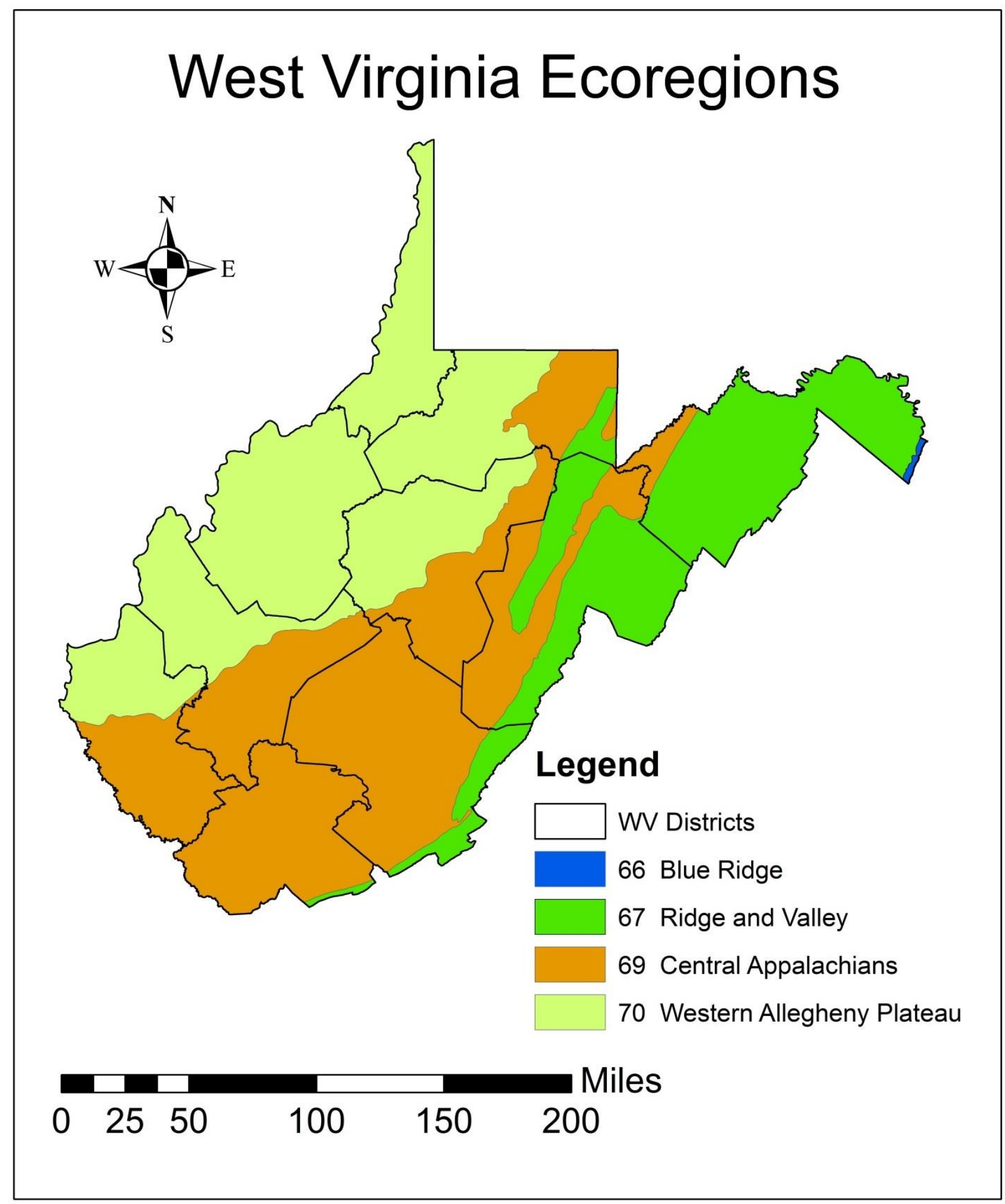

Figure 1: West Virginia Ecoregions 


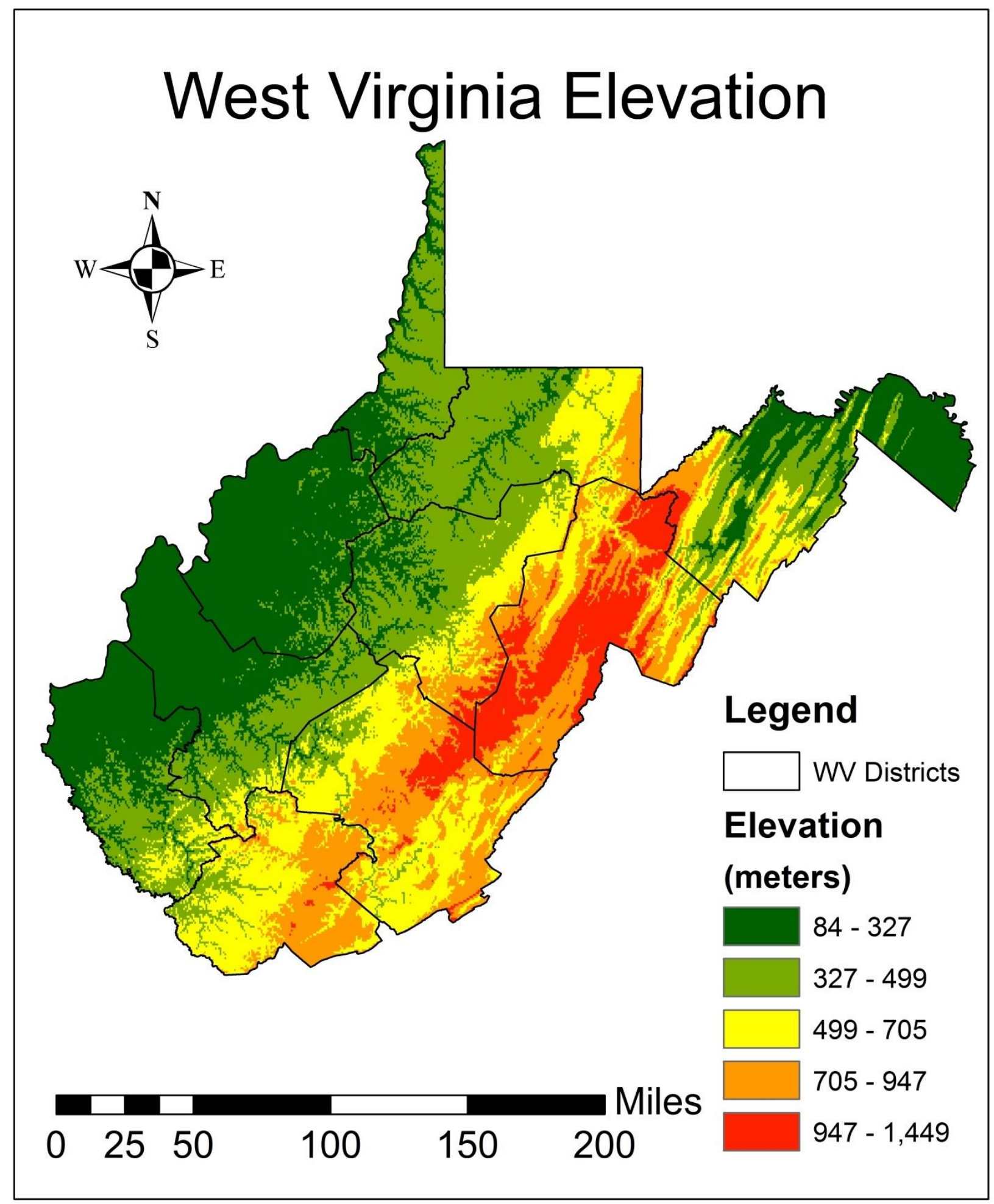

Figure 2: West Virginia Elevation (USGS NED 1/3 arc-second 2013 1x1 degree ArcGrid) courtesy of the U.S. Geological Survey 


\section{West Virginia Average Precipitation}

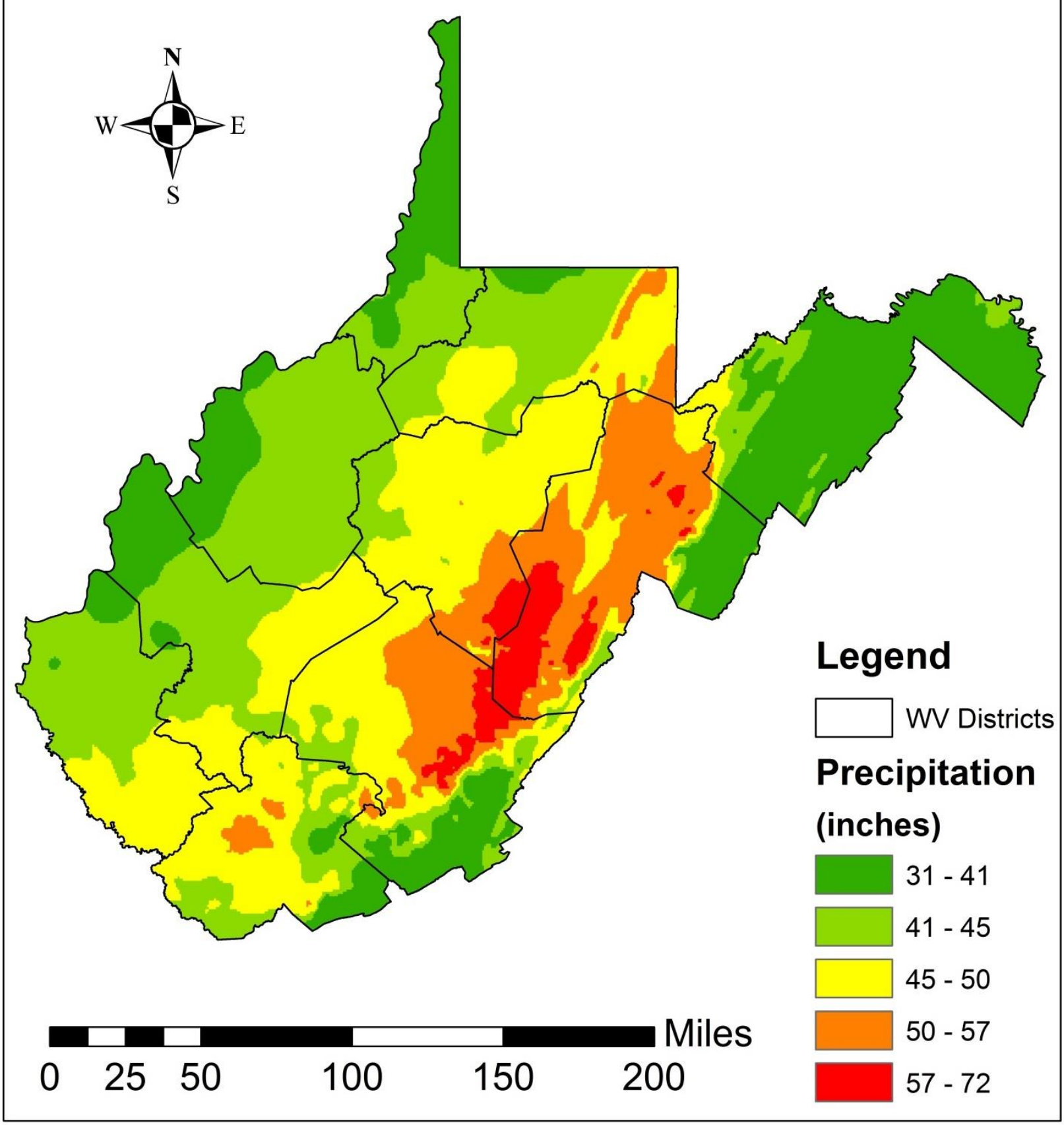

Figure 3: West Virginia Precipitation 


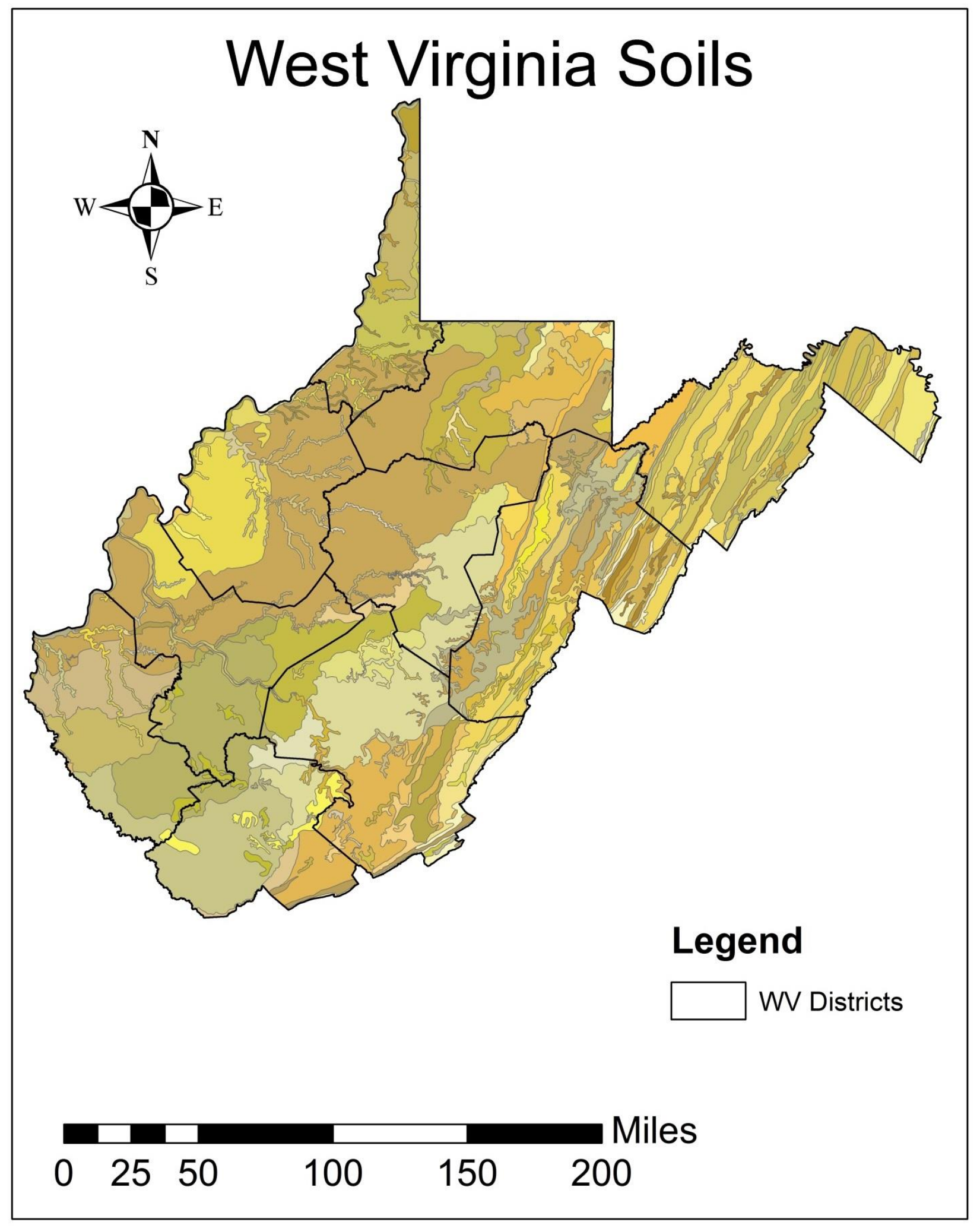

Figure 4: West Virginia Soils 


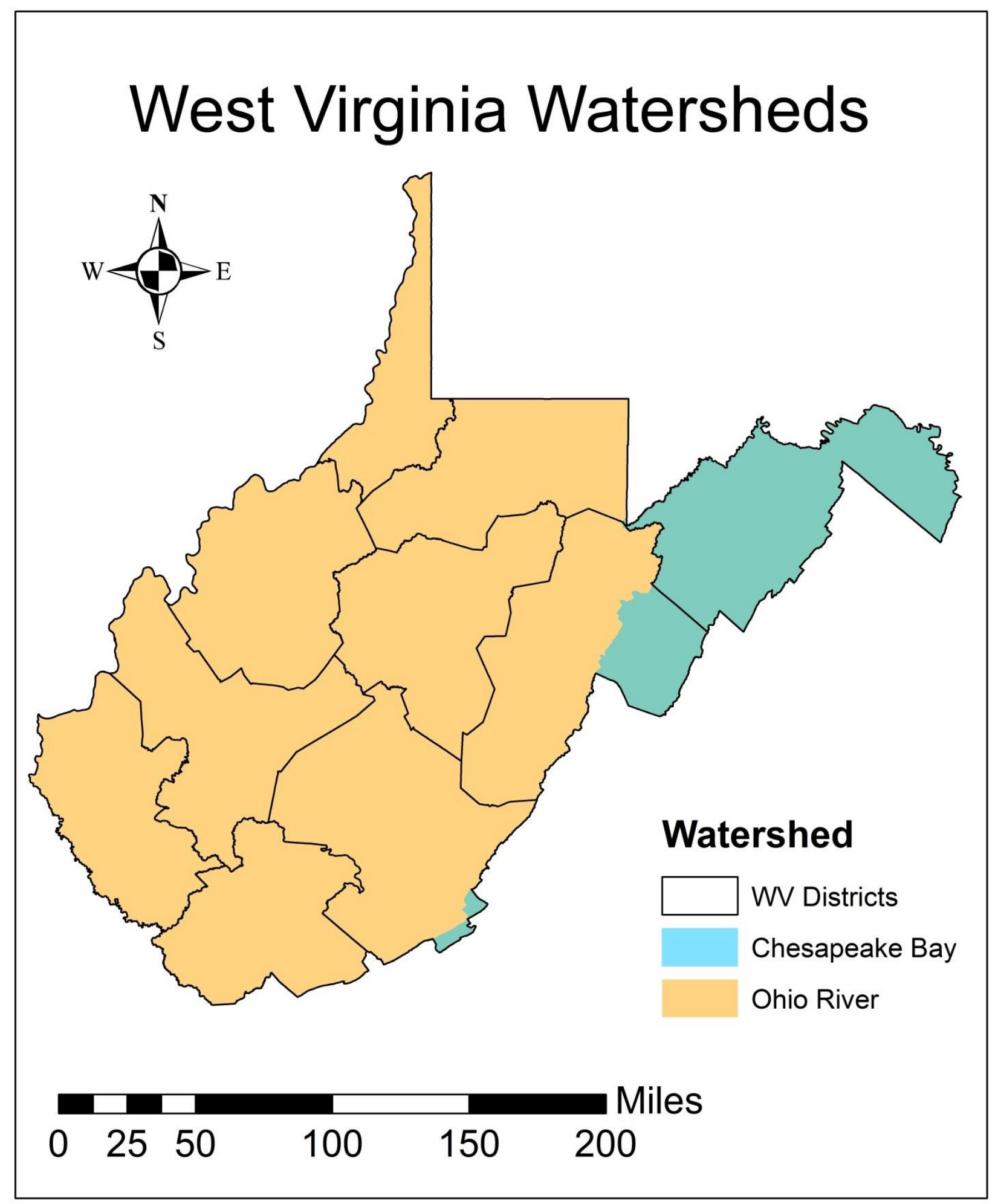

Figure 5: West Virginia Watersheds 


\subsubsection{Seeding Rates}

After the species were selected for the mixtures, the seeding rates for each species in the mixtures were determined. The USDA Plant Database (USDA, NRCS 2015), Ernst Seeds catalog (Ernst Conservation Seeds 2014), and an Ernst Seeds Horticulturist (Mark Fiely, personal communication, January 13-29, 2015) were consulted to determine appropriate seeding rates for these erosion control mixtures. Recommended seeding rates are displayed in Tables 1-6. Ernst Seeds horticulturist recommended $40 \mathrm{lb}(18.14 \mathrm{~kg})$ of grass seed for slopes greater than 3:1 H:V, a nurse crop in each native seed mixture, and 8-16 lb/ac (8.96- 17.92 kg/ha) PLS grass seed for highly compacted soils (Mark Fiely, personal communication, January $13-29,2015)$. Tables 1-6 display the seeding rates used to determine the final developed seed mixtures.

Table 1: Developed Type A- Mowable Areas Seeding Rates

\begin{tabular}{|c|c|c|c|c|}
\hline Scientific Name & Common Name & $\begin{array}{c}\text { USDA } \\
\text { (For a pure } \\
\text { stand) }\end{array}$ & $\begin{array}{l}\text { Ernst Seeds } \\
\text { Catalog (\% of } \\
\text { mixture) } \\
\end{array}$ & $\begin{array}{c}\text { Ernst } \\
\text { Horticulturist }\end{array}$ \\
\hline $\begin{array}{l}\text { Agrostis } \\
\text { perennans }\end{array}$ & Autumn Bentgrass & $\mathrm{N} / \mathrm{A}$ & $1 \%-25 \%$ & $\begin{array}{c}10 \mathrm{lb} / \mathrm{ac} \\
(11.2 \mathrm{~kg} / \mathrm{ha})\end{array}$ \\
\hline $\begin{array}{l}\text { Festuca rubra } \\
\text { ssp. commutata }\end{array}$ & Chewing's Fescue & $\mathrm{N} / \mathrm{A}$ & $\begin{array}{c}\text { Up to } 60 \mathrm{lb} / \mathrm{ac} \\
(67.19 \mathrm{~kg} / \mathrm{ha}) \text { of } \\
\text { a mixture }\end{array}$ & $\begin{array}{c}25 \mathrm{lb} / \mathrm{ac} \\
(28 \mathrm{~kg} / \mathrm{ha})\end{array}$ \\
\hline Festuca brevipila & $\begin{array}{l}\text { Hard Fescue } \\
\text { 'Chairot' }\end{array}$ & N/A & $30 \%$ & $\begin{array}{c}35 \mathrm{lb} / \mathrm{ac} \\
(39.2 \mathrm{~kg} / \mathrm{ha})\end{array}$ \\
\hline $\begin{array}{l}\text { Festuca ovina } \\
\text { var. duriuscula }\end{array}$ & $\begin{array}{l}\text { Hard Fescue } \\
\text { 'Heron' }\end{array}$ & N/A & $30 \%$ & $\begin{array}{c}35 \mathrm{lb} / \mathrm{ac} \\
(39.2 \mathrm{~kg} / \mathrm{ha})\end{array}$ \\
\hline Festuca rubra & $\begin{array}{l}\text { Creeping Red } \\
\text { Fescue }\end{array}$ & $\begin{array}{c}25 \%-60 \% \text { of } \\
\text { mixtures }\end{array}$ & $\begin{array}{l}\text { Up to } 60 \mathrm{lb} / \mathrm{ac} \\
(67.19 \mathrm{~kg} / \mathrm{ha}) \text { of } \\
\text { a mixture }\end{array}$ & $\begin{array}{c}55 \mathrm{lb} / \mathrm{ac} \\
(61.6 \mathrm{~kg} / \mathrm{ha})\end{array}$ \\
\hline Trifolium repens & $\begin{array}{l}\text { White Dutch } \\
\text { Clover }\end{array}$ & $\begin{array}{c}2 \mathrm{lb} / \mathrm{ac} \\
(2.24 \\
\mathrm{kg} / \mathrm{ha}) \mathrm{PLS}\end{array}$ & $10 \%$ & $\begin{array}{c}3 \mathrm{lb} / \mathrm{ac} \\
(3.4 \mathrm{~kg} / \mathrm{ha})\end{array}$ \\
\hline
\end{tabular}


Table 2: Developed Type B- Warm Season Seeding Rates

\begin{tabular}{|c|c|c|c|c|}
\hline Scientific Name & Common Name & $\begin{array}{c}\text { USDA } \\
\text { (For a pure } \\
\text { stand) }\end{array}$ & $\begin{array}{c}\text { Ernst Seeds } \\
\text { Catalog (\% of } \\
\text { mixture) }\end{array}$ & $\begin{array}{c}\text { Ernst } \\
\text { Horticulturist }\end{array}$ \\
\hline Avena sativa & $\begin{array}{l}\text { Common Oat } \\
\text { (Spring) }\end{array}$ & $\mathrm{N} / \mathrm{A}$ & $\begin{array}{c}30 \mathrm{lb} / \mathrm{ac} \\
(33.59 \mathrm{~kg} / \mathrm{ha})\end{array}$ & $\begin{array}{c}30 \mathrm{lb} / \mathrm{ac} \\
(33.59 \mathrm{~kg} / \mathrm{ha})\end{array}$ \\
\hline Secale cereale & Cereal Rye (Fall) & $\mathrm{N} / \mathrm{A}$ & $\begin{array}{c}30 \mathrm{lb} / \mathrm{ac} \\
(33.59 \mathrm{~kg} / \mathrm{ha})\end{array}$ & $\begin{array}{c}30 \mathrm{lb} / \mathrm{ac} \\
(33.59 \mathrm{~kg} / \mathrm{ha})\end{array}$ \\
\hline $\begin{array}{l}\text { Andropogon } \\
\text { gerardii }\end{array}$ & Big Bluestem & $\begin{array}{c}10 \mathrm{lb} / \mathrm{ac} \\
(11.20 \\
\mathrm{kg} / \mathrm{ha}) \text { PLS }\end{array}$ & $5 \%-50 \%$ & $\begin{array}{c}6 \mathrm{lb} / \mathrm{ac} \\
(6.7 \mathrm{~kg} / \mathrm{ha})\end{array}$ \\
\hline $\begin{array}{l}\text { Elymus } \\
\text { virginicus }\end{array}$ & Virginia Wildrye & $\begin{array}{c}20 \mathrm{lb} / \mathrm{ac} \\
(22.40 \\
\mathrm{kg} / \mathrm{ha}) \mathrm{PLS}\end{array}$ & $1 \%-25 \%$ & $\begin{array}{c}8.4 \mathrm{lb} / \mathrm{ac} \\
(9.4 \mathrm{~kg} / \mathrm{ha})\end{array}$ \\
\hline $\begin{array}{l}\text { Agrostis } \\
\text { perennans }\end{array}$ & Autumn Bentgrass & N/A & $1 \%-25 \%$ & $\begin{array}{c}3.7 \mathrm{lb} / \mathrm{ac} \\
(4.1 \mathrm{~kg} / \mathrm{ha})\end{array}$ \\
\hline $\begin{array}{l}\text { Panicum } \\
\text { virgatum }\end{array}$ & Switchgrass & $\begin{array}{l}7.5-15 \mathrm{lb} / \mathrm{ac} \\
(6.72-13.44 \\
\mathrm{kg} / \mathrm{ha}) \text { PLS }\end{array}$ & $10 \%-20 \%$ & $\begin{array}{c}6.4 \mathrm{lb} / \mathrm{ac} \\
(7.2 \mathrm{~kg} / \mathrm{ha})\end{array}$ \\
\hline $\begin{array}{l}\text { Sorghastrum } \\
\text { nutans }\end{array}$ & Indiangrass & $\begin{array}{c}10 \mathrm{lb} / \mathrm{ac} \\
(11.20 \\
\mathrm{kg} / \mathrm{ha}) \mathrm{PLS}\end{array}$ & $10 \%-50 \%$ & $\begin{array}{c}14 \mathrm{lb} / \mathrm{ac} \\
(15.7 \mathrm{~kg} / \mathrm{ha})\end{array}$ \\
\hline $\begin{array}{l}\text { Chamaecrista } \\
\text { fasciculata }\end{array}$ & Partridge Pea & $\begin{array}{c}10 \mathrm{lb} / \mathrm{ac} \\
(11.20 \\
\mathrm{kg} / \mathrm{ha}) \mathrm{PLS}\end{array}$ & $1 \%-10 \%$ & $\begin{array}{c}1 \mathrm{lb} / \mathrm{ac} \\
(1.1 \mathrm{~kg} / \mathrm{ha})\end{array}$ \\
\hline Rudbeckia hirta & Black-Eyed Susan & $\begin{array}{c}0.5 \mathrm{lb} / \mathrm{ac} \\
(0.56 \mathrm{~kg} / \mathrm{ha}) \\
\mathrm{PLS}\end{array}$ & $1 \%-5 \%$ & $\begin{array}{c}0.6 \mathrm{lb} / \mathrm{ac} \\
(0.7 \mathrm{~kg} / \mathrm{ha})\end{array}$ \\
\hline $\begin{array}{l}\text { Pycnanthemum } \\
\text { tenuifolium }\end{array}$ & $\begin{array}{l}\text { Narrowleaf } \\
\text { Mountainmint }\end{array}$ & $\mathrm{N} / \mathrm{A}$ & $1 \%$ & $\begin{array}{c}1 \mathrm{lb} / \mathrm{ac} \\
(1.1 \mathrm{~kg} / \mathrm{ha})\end{array}$ \\
\hline $\begin{array}{l}\text { Monarda } \\
\text { fistulosa }\end{array}$ & Wild Bergamot & $N / A$ & $1 \%-2 \%$ & $\begin{array}{c}0.5 \mathrm{lb} / \mathrm{ac} \\
(0.6 \mathrm{~kg} / \mathrm{ha})\end{array}$ \\
\hline $\begin{array}{l}\text { Desmodium } \\
\text { paniculatum }\end{array}$ & $\begin{array}{l}\text { Panicledleaf } \\
\text { Ticktrefoil }\end{array}$ & $\mathrm{N} / \mathrm{A}$ & $1 \%-5 \%$ & $\begin{array}{c}0.4 \mathrm{lb} / \mathrm{ac} \\
(0.4 \mathrm{~kg} / \mathrm{ha})\end{array}$ \\
\hline $\begin{array}{l}\text { Heliopsis } \\
\text { helianthoides }\end{array}$ & Smooth Oxeye & $\mathrm{N} / \mathrm{A}$ & $1 \%-5 \%$ & $\begin{array}{c}0.4 \mathrm{lb} / \mathrm{ac} \\
(0.4 \mathrm{~kg} / \mathrm{ha})\end{array}$ \\
\hline $\begin{array}{l}\text { Euthamia } \\
\text { graminifolia }\end{array}$ & Flat-top Goldentop & $\mathrm{N} / \mathrm{A}$ & $1 \%$ & $\begin{array}{c}1 \mathrm{lb} / \mathrm{ac} \\
(1.1 \mathrm{~kg} / \mathrm{ha})\end{array}$ \\
\hline
\end{tabular}


Table 3: Developed Type C- Cool Season Seeding Rates

\begin{tabular}{|c|c|c|c|c|}
\hline $\begin{array}{l}\text { Scientific } \\
\text { Name }\end{array}$ & Common Name & $\begin{array}{c}\text { USDA } \\
\text { (For a pure } \\
\text { stand) }\end{array}$ & $\begin{array}{c}\text { Ernst Seeds } \\
\text { Catalog } \\
\text { (\% of mixture) }\end{array}$ & $\begin{array}{c}\text { Ernst } \\
\text { Horticulturist }\end{array}$ \\
\hline $\begin{array}{l}\text { Agrostis } \\
\text { gigantea }\end{array}$ & Red Top & $\begin{array}{c}1-2 \mathrm{lb} / \mathrm{ac} \\
(1.12-2.24 \\
\mathrm{kg} / \mathrm{ha}) \\
\mathrm{PLS} \text { in } \\
\text { mixtures }\end{array}$ & $5 \%-25 \%$ & $\begin{array}{c}4 \mathrm{lb} / \mathrm{ac} \\
(4.5 \mathrm{~kg} / \mathrm{ha})\end{array}$ \\
\hline $\begin{array}{l}\text { Festuca ovina } \\
\text { var. duriuscula }\end{array}$ & $\begin{array}{l}\text { Hard Fescue } \\
\text { 'Heron' }\end{array}$ & $\mathrm{N} / \mathrm{A}$ & $30 \%$ & $\begin{array}{c}10 \mathrm{lb} / \mathrm{ac} \\
(11.2 \mathrm{~kg} / \mathrm{ha})\end{array}$ \\
\hline $\begin{array}{l}\text { Festuca } \\
\text { brevipila }\end{array}$ & $\begin{array}{l}\text { Hard Fescue } \\
\text { 'Chariot' }\end{array}$ & $N / A$ & $30 \%$ & $\begin{array}{c}10 \mathrm{lb} / \mathrm{ac} \\
(11.2 \mathrm{~kg} / \mathrm{ha})\end{array}$ \\
\hline Festuca rubra & $\begin{array}{l}\text { Creeping Red } \\
\text { Fescue }\end{array}$ & $\begin{array}{c}25 \%-60 \% \text { of } \\
\text { mixtures }\end{array}$ & $\begin{array}{l}\text { Up to } 60 \mathrm{lb} / \mathrm{ac} \\
(67.19 \mathrm{~kg} / \mathrm{ha}) \\
\text { of a mixture }\end{array}$ & $\begin{array}{c}16 \mathrm{lb} / \mathrm{ac} \\
(17.9 \mathrm{~kg} / \mathrm{ha})\end{array}$ \\
\hline $\begin{array}{l}\text { Trifolium } \\
\text { repens }\end{array}$ & White Clover & $\begin{array}{c}2 \mathrm{lb} / \mathrm{ac}(2.24 \\
\mathrm{kg} / \mathrm{ha}) \text { PLS }\end{array}$ & $10 \%$ & $\begin{array}{c}7 \mathrm{lb} / \mathrm{ac} \\
(7.8 \mathrm{~kg} / \mathrm{ha})\end{array}$ \\
\hline $\begin{array}{l}\text { Lotus } \\
\text { corniculatus }\end{array}$ & Birdsfoot Trefoil & $\begin{array}{l}4-8 \mathrm{lb} / \mathrm{ac} \\
(4.48-8.96 \\
\mathrm{kg} / \mathrm{ha}) \text { for } \\
\text { pastures }\end{array}$ & $\begin{array}{c}8-15 \mathrm{lb} / \mathrm{ac} \\
(8.96-16.80 \\
\mathrm{kg} / \mathrm{ha}) \\
\text { of a mixture }\end{array}$ & $\begin{array}{c}8 \mathrm{lb} / \mathrm{ac} \\
(9 \mathrm{~kg} / \mathrm{ha})\end{array}$ \\
\hline
\end{tabular}


Table 4: Developed Type D1- High Elevation Seeding Rates

\begin{tabular}{|c|c|c|c|c|}
\hline Scientific Name & Common Name & $\begin{array}{c}\text { USDA } \\
\text { (For a pure } \\
\text { stand) }\end{array}$ & $\begin{array}{c}\text { Ernst Seeds } \\
\text { Catalog } \\
\text { (\% of mixture) }\end{array}$ & $\begin{array}{c}\text { Ernst } \\
\text { Horticulturist }\end{array}$ \\
\hline Avena sativa & $\begin{array}{l}\text { Common Oat } \\
\text { (Spring) }\end{array}$ & $N / A$ & $\begin{array}{c}30 \mathrm{lb} / \mathrm{ac} \\
(33.59 \mathrm{~kg} / \mathrm{ha})\end{array}$ & $\begin{array}{c}30 \mathrm{lb} / \mathrm{ac} \\
(33.59 \mathrm{~kg} / \mathrm{ha})\end{array}$ \\
\hline Secale cereale & Cereal Rye (Fall) & $\mathrm{N} / \mathrm{A}$ & $\begin{array}{c}30 \mathrm{lb} / \mathrm{ac} \\
(33.59 \mathrm{~kg} / \mathrm{ha})\end{array}$ & $\begin{array}{c}30 \mathrm{lb} / \mathrm{ac} \\
(33.59 \mathrm{~kg} / \mathrm{ha})\end{array}$ \\
\hline $\begin{array}{l}\text { Andropogon } \\
\text { gerardii }\end{array}$ & Big Bluestem & $\begin{array}{c}10 \mathrm{lb} / \mathrm{ac} \\
(11.20 \mathrm{~kg} / \mathrm{ha}) \\
\text { PLS }\end{array}$ & $5 \%-50 \%$ & $\begin{array}{c}2.5 \mathrm{lb} / \mathrm{ac} \\
(2.8 \mathrm{~kg} / \mathrm{ha})\end{array}$ \\
\hline Elymus virginicus & Virginia Wildrye & $\begin{array}{c}20 \mathrm{lb} / \mathrm{ac} \\
(22.40 \mathrm{~kg} / \mathrm{ha}) \\
\text { PLS }\end{array}$ & $1 \%-25 \%$ & $\begin{array}{c}4 \mathrm{lb} / \mathrm{ac} \\
(4.5 \mathrm{~kg} / \mathrm{ha})\end{array}$ \\
\hline $\begin{array}{l}\text { Agrostis } \\
\text { perennans }\end{array}$ & Autumn Bentgrass & $N / A$ & $1 \%-25 \%$ & $\begin{array}{c}6.7 \mathrm{lb} / \mathrm{ac} \\
(7.5 \mathrm{~kg} / \mathrm{ha})\end{array}$ \\
\hline $\begin{array}{l}\text { Dichanthelium } \\
\text { clandestinum }\end{array}$ & Deertongue & $\begin{array}{c}3 \mathrm{lb} / \mathrm{ac}(3.36 \\
\mathrm{kg} / \mathrm{ha}) \text { PLS } \\
\text { for general } \\
\text { mixtures }\end{array}$ & $1 \%-25 \%$ & $\begin{array}{c}0.5-8 \mathrm{lb} / \mathrm{ac} \\
(0.6-9 \mathrm{~kg} / \mathrm{ha})\end{array}$ \\
\hline $\begin{array}{l}\text { Schizachyrium } \\
\text { scoparium }\end{array}$ & Partridge Pea & $\begin{array}{c}10 \mathrm{lb} / \mathrm{ac} \\
(11.20 \mathrm{~kg} / \mathrm{ha}) \\
\text { PLS }\end{array}$ & $1 \%-10 \%$ & $\begin{array}{c}1 \mathrm{lb} / \mathrm{ac} \\
(1.1 \mathrm{~kg} / \mathrm{ha})\end{array}$ \\
\hline $\begin{array}{l}\text { Chamaecrista } \\
\text { fasciculata }\end{array}$ & Black-Eyed Susan & $\begin{array}{c}0.5 \mathrm{lb} / \mathrm{ac} \\
(0.56 \mathrm{~kg} / \mathrm{ha}) \\
\text { PLS }\end{array}$ & $1 \%-5 \%$ & $\begin{array}{c}0.6 \mathrm{lb} / \mathrm{ac} \\
(0.7 \mathrm{~kg} / \mathrm{ha})\end{array}$ \\
\hline Rudbeckia hirta & Smooth Oxeye & $\mathrm{N} / \mathrm{A}$ & $1 \%-5 \%$ & $\begin{array}{c}0.4 \mathrm{lb} / \mathrm{ac} \\
(0.4 \mathrm{~kg} / \mathrm{ha})\end{array}$ \\
\hline $\begin{array}{l}\text { Heliopsis } \\
\text { helianthoides }\end{array}$ & $\begin{array}{l}\text { Wrinkleleaf } \\
\text { Goldenrod }\end{array}$ & $\mathrm{N} / \mathrm{A}$ & $1 \%-2 \%$ & $\begin{array}{c}0.2 \mathrm{lb} / \mathrm{ac} \\
(0.2 \mathrm{~kg} / \mathrm{ha})\end{array}$ \\
\hline Solidago rugosa & $\begin{array}{l}\text { Narrowleaf } \\
\text { Mountainmint }\end{array}$ & $\mathrm{N} / \mathrm{A}$ & $1 \%$ & $\begin{array}{c}0.1 \mathrm{lb} / \mathrm{ac} \\
(0.1 \mathrm{~kg} / \mathrm{ha})\end{array}$ \\
\hline $\begin{array}{l}\text { Pycnanthemum } \\
\text { tenuifolium }\end{array}$ & $\begin{array}{l}\text { Panicledleaf } \\
\text { Ticktrefoil }\end{array}$ & $\mathrm{N} / \mathrm{A}$ & $1 \%-5 \%$ & $\begin{array}{c}0.4 \mathrm{lb} / \mathrm{ac} \\
(0.4 \mathrm{~kg} / \mathrm{ha})\end{array}$ \\
\hline $\begin{array}{l}\text { Desmodium } \\
\text { paniculatum }\end{array}$ & Flat-top Goldentop & $\mathrm{N} / \mathrm{A}$ & $1 \%$ & $\begin{array}{c}0.1 \mathrm{lb} / \mathrm{ac} \\
(0.1 \mathrm{~kg} / \mathrm{ha}) \\
\end{array}$ \\
\hline
\end{tabular}


Table 5: Developed Type D2- High Elevation Seeding Rates

\begin{tabular}{|c|c|c|c|c|}
\hline $\begin{array}{l}\text { Scientific } \\
\text { Name }\end{array}$ & Common Name & $\begin{array}{c}\text { USDA } \\
\text { (For a pure } \\
\text { stand) }\end{array}$ & $\begin{array}{c}\text { Ernst Seeds } \\
\text { Catalog } \\
\text { (\% of mixture) }\end{array}$ & $\begin{array}{c}\text { Ernst } \\
\text { Horticulturist }\end{array}$ \\
\hline Avena sativa & $\begin{array}{l}\text { Common Oat } \\
\text { (Spring) }\end{array}$ & $\mathrm{N} / \mathrm{A}$ & $\begin{array}{c}30 \mathrm{lb} / \mathrm{ac} \\
(33.59 \mathrm{~kg} / \mathrm{ha})\end{array}$ & $\begin{array}{c}30 \mathrm{lb} / \mathrm{ac} \\
(33.59 \mathrm{~kg} / \mathrm{ha})\end{array}$ \\
\hline Secale cereale & Cereal Rye (Fall) & $\mathrm{N} / \mathrm{A}$ & $\begin{array}{c}30 \mathrm{lb} / \mathrm{ac} \\
(33.59 \mathrm{~kg} / \mathrm{ha})\end{array}$ & $\begin{array}{c}30 \mathrm{lb} / \mathrm{ac} \\
(33.59 \mathrm{~kg} / \mathrm{ha})\end{array}$ \\
\hline $\begin{array}{l}\text { Andropogon } \\
\text { gerardii }\end{array}$ & Big Bluestem & $\begin{array}{c}10 \mathrm{lb} / \mathrm{ac} \\
(11.20 \mathrm{~kg} / \mathrm{ha}) \\
\text { PLS }\end{array}$ & $5 \%-50 \%$ & $\begin{array}{c}8 \mathrm{lb} / \mathrm{ac} \\
(8.9 \mathrm{~kg} / \mathrm{ha})\end{array}$ \\
\hline $\begin{array}{l}\text { Elymus } \\
\text { virginicus }\end{array}$ & Virginia Wildrye & $\begin{array}{c}20 \mathrm{lb} / \mathrm{ac} \\
(22.40 \mathrm{~kg} / \mathrm{ha}) \\
\text { PLS }\end{array}$ & $1 \%-25 \%$ & $\begin{array}{c}8.2 \mathrm{lb} / \mathrm{ac} \\
(9.2 \mathrm{~kg} / \mathrm{ha})\end{array}$ \\
\hline $\begin{array}{l}\text { Agrostis } \\
\text { perennans }\end{array}$ & Autumn Bentgrass & $N / A$ & $1 \%-25 \%$ & $\begin{array}{c}14 \mathrm{lb} / \mathrm{ac} \\
(15.7 \mathrm{~kg} / \mathrm{ha})\end{array}$ \\
\hline $\begin{array}{l}\text { Dichanthelium } \\
\text { clandestinum }\end{array}$ & Deertongue & $\begin{array}{l}3 \mathrm{lb} / \mathrm{ac}(3.36 \\
\mathrm{kg} / \mathrm{ha}) \text { PLS } \\
\text { for general } \\
\text { mixtures }\end{array}$ & $1 \%-25 \%$ & $\begin{array}{c}10 \mathrm{lb} / \mathrm{ac} \\
(11.20 \mathrm{~kg} / \mathrm{ha})\end{array}$ \\
\hline $\begin{array}{l}\text { Schizachyrium } \\
\text { scoparium }\end{array}$ & Partridge Pea & $\begin{array}{c}10 \mathrm{lb} / \mathrm{ac} \\
(11.20 \mathrm{~kg} / \mathrm{ha}) \\
\text { PLS }\end{array}$ & $1 \%-10 \%$ & $\begin{array}{c}1 \mathrm{lb} / \mathrm{ac} \\
(1.1 \mathrm{~kg} / \mathrm{ha})\end{array}$ \\
\hline $\begin{array}{l}\text { Chamaecrista } \\
\text { fasciculata }\end{array}$ & Black-Eyed Susan & $\begin{array}{c}0.5 \mathrm{lb} / \mathrm{ac} \\
(0.56 \mathrm{~kg} / \mathrm{ha}) \\
\text { PLS }\end{array}$ & $1 \%-5 \%$ & $\begin{array}{c}0.6 \mathrm{lb} / \mathrm{ac} \\
(0.7 \mathrm{~kg} / \mathrm{ha})\end{array}$ \\
\hline Rudbeckia hirta & Smooth Oxeye & $N / A$ & $1 \%-5 \%$ & $\begin{array}{c}0.4 \mathrm{lb} / \mathrm{ac} \\
(0.4 \mathrm{~kg} / \mathrm{ha})\end{array}$ \\
\hline $\begin{array}{l}\text { Heliopsis } \\
\text { helianthoides }\end{array}$ & $\begin{array}{l}\text { Wrinkleleaf } \\
\text { Goldenrod }\end{array}$ & $\mathrm{N} / \mathrm{A}$ & $1 \%-2 \%$ & $\begin{array}{c}0.2 \mathrm{lb} / \mathrm{ac} \\
(0.2 \mathrm{~kg} / \mathrm{ha})\end{array}$ \\
\hline Solidago rugosa & $\begin{array}{l}\text { Narrowleaf } \\
\text { Mountainmint }\end{array}$ & $\mathrm{N} / \mathrm{A}$ & $1 \%$ & $\begin{array}{c}0.1 \mathrm{lb} / \mathrm{ac} \\
(0.1 \mathrm{~kg} / \mathrm{ha})\end{array}$ \\
\hline $\begin{array}{l}\text { Pycnanthemum } \\
\text { tenuifolium }\end{array}$ & $\begin{array}{l}\text { Panicledleaf } \\
\text { Ticktrefoil }\end{array}$ & $\mathrm{N} / \mathrm{A}$ & $1 \%-5 \%$ & $\begin{array}{c}0.4 \mathrm{lb} / \mathrm{ac} \\
(0.4 \mathrm{~kg} / \mathrm{ha})\end{array}$ \\
\hline $\begin{array}{l}\text { Desmodium } \\
\text { paniculatum }\end{array}$ & Flat-top Goldentop & $\mathrm{N} / \mathrm{A}$ & $1 \%$ & $\begin{array}{c}0.1 \mathrm{lb} / \mathrm{ac} \\
(0.1 \mathrm{~kg} / \mathrm{ha}) \\
\end{array}$ \\
\hline
\end{tabular}


Table 6: Developed Type E- Wet Areas Seeding Rates

\begin{tabular}{|c|c|c|c|c|}
\hline Scientific Name & Common Name & $\begin{array}{c}\text { USDA } \\
\text { (For a pure } \\
\text { stand) }\end{array}$ & $\begin{array}{c}\text { Ernst Seeds } \\
\text { Catalog } \\
\text { (\% of mixture) }\end{array}$ & $\begin{array}{c}\text { Ernst } \\
\text { Horticulturist }\end{array}$ \\
\hline Carex vulpinoidea & Fox Sedge & $\mathrm{N} / \mathrm{A}$ & $1 \%-30 \%$ & $\begin{array}{c}4 \mathrm{lb} / \mathrm{ac} \\
(4.5 \mathrm{~kg} / \mathrm{ha})\end{array}$ \\
\hline Poa palustris & Fowl Bluegrass & $\mathrm{N} / \mathrm{A}$ & $5 \%-25 \%$ & $\begin{array}{c}3.5 \mathrm{lb} / \mathrm{ac} \\
(3.9 \mathrm{~kg} / \mathrm{ha})\end{array}$ \\
\hline Panicum rigidulum & $\begin{array}{l}\text { Redtop } \\
\text { Panicgrass }\end{array}$ & $\mathrm{N} / \mathrm{A}$ & $1 \%-50 \%$ & $\begin{array}{c}4 \mathrm{lb} / \mathrm{ac} \\
(4.5 \mathrm{~kg} / \mathrm{ha})\end{array}$ \\
\hline Juncus effusus & Common Rush & $\mathrm{N} / \mathrm{A}$ & $1 \%-5 \%$ & $\begin{array}{c}0.5 \mathrm{lb} / \mathrm{ac} \\
(0.5 \mathrm{~kg} / \mathrm{ha})\end{array}$ \\
\hline Elymus virginicus & Virginia Wild Rye & $\begin{array}{c}20 \mathrm{lb} / \mathrm{ac} \\
(22.4 \mathrm{~kg} / \mathrm{ha}) \\
\text { PLS }\end{array}$ & $1 \%-25 \%$ & $\begin{array}{c}4 \mathrm{lb} / \mathrm{ac} \\
(4.5 \mathrm{~kg} / \mathrm{ha})\end{array}$ \\
\hline Carex lurida & Shallow Sedge & $\mathrm{N} / \mathrm{A}$ & $1 \%-20 \%$ & $\begin{array}{c}3 \mathrm{lb} / \mathrm{ac} \\
(3.4 \mathrm{~kg} / \mathrm{ha})\end{array}$ \\
\hline Verbena hastata & Blue Vervain & $\mathrm{N} / \mathrm{A}$ & $1 \%-10 \%$ & $\begin{array}{c}0.8 \mathrm{lb} / \mathrm{ac} \\
(0.9 \mathrm{~kg} / \mathrm{ha})\end{array}$ \\
\hline $\begin{array}{l}\text { Verbesina } \\
\text { alternifolia }\end{array}$ & Wingstem & $\mathrm{N} / \mathrm{A}$ & $1 \%-2 \%$ & $\begin{array}{c}0.2 \mathrm{lb} / \mathrm{ac} \\
(0.2 \mathrm{~kg} / \mathrm{ha})\end{array}$ \\
\hline
\end{tabular}

\subsection{Results}

\subsubsection{GIS Maps}

Species selection was limited to species that perform both well and frequently in West Virginia. Figures 1-5 depict West Virginia's ecoregions, elevations, precipitation, soils, and watersheds. This information was considered during seed species selection and examination.

Figure 1 displays U.S. EPA Level III Ecoregions for the state of West Virginia (U.S. Environmental Protection Agency 2013). The state has four ecoregions consisting of Blue Ridge, Ridge and Valley, Central Appalachians, and Western Allegheny Plateau. The Blue Ridge ecoregion is the smallest of the four occupying the extreme eastern part of the state and was not taken into consideration in the seed selection process.

Figure 2 illustrates the various elevations throughout West Virginia. The map has five categories of elevation ranges to distinguish between the major elevation changes in the state. The average elevation of West Virginia is $1,500 \mathrm{ft}(460 \mathrm{~m})$ with the highest elevation at Spruce 
Knob, 4,863 ft (1,482 m). The lowest recorded elevation is $240 \mathrm{ft}(73 \mathrm{~m})$ at Harpers Ferry along the Potomac River.

Figure 3 presents the average precipitation for the state. There are five category ranges to display the major precipitation changes in West Virginia. The lowest average precipitation is $2.58 \mathrm{ft}(0.78 \mathrm{~m})$ and the highest is $6 \mathrm{ft}(1.8 \mathrm{~m})$. For Figure 4, various soil types are displayed in varying colors to illustrate the variance in soil media throughout West Virginia.

Figure 5 presents the watersheds of West Virginia. The state has two major watersheds. The largest is the Ohio River which occupies almost everything west of the Eastern panhandle. Chesapeake Bay is the second watershed and it encompasses the Eastern panhandle and one small section in the southeast part of the state.

\subsubsection{Seed Mixtures}

Six seed mixtures were developed. The seed mixtures are as follows:

- Developed Type A: Mowable Areas

- Developed Type B: Warm Season

- Developed Type C: Cool Season

- Developed Type D1: High Elevation (>2,400 ft, $731.52 \mathrm{~m})(\leq 3: 1 \mathrm{H}: \mathrm{V})$

- Developed Type D2: High Elevation (>2,400 ft, $731.52 \mathrm{~m})(>3: 1 \mathrm{H}: \mathrm{V})$

- Developed Type E: Wet Areas

The six seed mixtures were developed for WV. The seed mixtures are detailed in the following sections.

\section{Developed Type A: Mowable Areas}

Developed Type A mixture was intended for areas that would experience frequent mowing and would be highly visible to highway corridor users. Low growth, erosion control, fast establishment, and visual appeal were the main traits considered. Due to the low mowing height presently used by the WVDOH, a non-native dominated seed mixture had to be used. Autumn 
bentgrass (Agrostis perennans), chewing's fescue (Festuca rubra ssp. commutata), hard fescues 'Chariot' (Festuca brevipila) and 'Heron' (Festuca ovina var. duriuscula), creeping red fescue (Festuca rubra), and white clover (Trifolium repens) were the chosen species (Table 7). Table 8 displays the species occurrence in West Virginia according to count by county, ecoregion, elevation ranges, and watersheds.

Autumn bentgrass was chosen because the species grows to a height approximately 3.3 $\mathrm{ft}(1 \mathrm{~m})$ in damp to dry soils and areas of full to partial sunlight. The species is also a native graminoid, but does not withstand salt and drought (Ernst Conservation Seeds 2014). Chewing's fescue was selected because the species is used for erosion control. This graminoid is a non-native bunchgrass species that can grow to a height of 2-3 $\mathrm{ft}$ ( 0.61 to $0.91 \mathrm{~m}$ )(Ernst Conservation Seeds 2014). The hard fescue species are perennial cool season bunchgrasses that grow to a height of $2.5 \mathrm{ft}(0.76 \mathrm{~m})$. The species is frequently used for erosion control in recreational areas and lawns and along roadways. This graminoid is persistent, drought resistant, and has a long life span (Crowder N.D.). Creeping red fescue is a cool season bunchgrass that has stems that grow to a height of $2 \mathrm{ft}(0.61 \mathrm{~m})$. This graminoid was chosen for its ability to bind the soil and stabilize a site. The species is also frequently used in turf applications because it is resistant to wear, resilient, and tolerant to shade (USDA- NRCS 2002e). White clover is a perennial legume with flowering heads. It may develop an invasive nature. The species was chosen to fixate nitrogen to the soil and act as an erosion control cover. This legume is presently used as an erosion control species (USDA- NRCS 2002f). 
Table 7: Developed Type A-Mowable Areas Seed Mixture; Planting time periods: Spring (March 1st - June 20th) and (August 1st - October 31st) for Summer and Fall

\begin{tabular}{|c|c|c|c|c|c|c|}
\hline $\begin{array}{l}\text { Scientific } \\
\text { Name }\end{array}$ & $\begin{array}{l}\text { Common } \\
\text { Name }\end{array}$ & $\begin{array}{c}\text { Rate } \\
\text { (lb/ac) }\end{array}$ & $\begin{array}{l}\text { Cost } \\
(/ / \mathrm{lb})\end{array}$ & Classification & Description & $\begin{array}{l}\text { Threat } \\
\text { Level }\end{array}$ \\
\hline $\begin{array}{l}\text { Agrostis } \\
\text { perennans }\end{array}$ & $\begin{array}{l}\text { Autumn } \\
\text { Bentgrass }\end{array}$ & 10 & $\$ 14$ & Native & Graminoid & $\mathrm{N} / \mathrm{A}$ \\
\hline $\begin{array}{l}\text { Festuca } \\
\text { rubra ssp. } \\
\text { commutata }\end{array}$ & $\begin{array}{l}\text { Chewing's } \\
\text { Fescue }\end{array}$ & 25 & $\$ 2.10$ & Non-Native & Graminoid & Introduced \\
\hline $\begin{array}{l}\text { Festuca } \\
\text { brevipila }\end{array}$ & $\begin{array}{l}\text { Hard Fescue } \\
\text { 'Chariot' }\end{array}$ & 35 & $\$ 2.80$ & Non-Native & Graminoid & Introduced \\
\hline $\begin{array}{l}\text { Festuca } \\
\text { ovina var. } \\
\text { duriuscula }\end{array}$ & $\begin{array}{l}\text { Hard Fescue } \\
\text { 'Heron' }\end{array}$ & 35 & $\$ 2.80$ & Non-Native & Graminoid & Introduced \\
\hline $\begin{array}{l}\text { Festuca } \\
\text { rubra }\end{array}$ & $\begin{array}{l}\text { Creeping Red } \\
\text { Fescue }\end{array}$ & 55 & $\$ 1.50$ & Non-Native & Graminoid & Introduced \\
\hline \multirow[t]{2}{*}{$\begin{array}{l}\text { Trifolium } \\
\text { repens }\end{array}$} & White Clover & 3 & $\$ 3.80$ & Non-Native & Legume & 3 \\
\hline & Total: & 163 & $\$ 2.96$ & $\$ 482.40 /$ acre & & \\
\hline
\end{tabular}

Table 8: Mowable Areas Species Occurrence

\begin{tabular}{|c|c|c|c|c|c|}
\hline $\begin{array}{l}\text { Scientific } \\
\text { Name }\end{array}$ & Common Name & $\begin{array}{c}\text { Occurrence } \\
\text { (out of } 55 \\
\text { counties) }\end{array}$ & $\begin{array}{c}\text { Ecoregions } \\
\text { (out of } 3 \\
\text { regions) }\end{array}$ & $\begin{array}{l}\text { Elevation } \\
\text { (out of } 5 \\
\text { ranges) }\end{array}$ & $\begin{array}{c}\text { Watershed } \\
\text { (out of } 2 \\
\text { watersheds) }\end{array}$ \\
\hline $\begin{array}{l}\text { Agrostis } \\
\text { perennans }\end{array}$ & $\begin{array}{l}\text { Autumn } \\
\text { Bentgrass }\end{array}$ & 32 & 3 & 5 & 2 \\
\hline $\begin{array}{l}\text { Festuca } \\
\text { rubra ssp. } \\
\text { commutata }\end{array}$ & $\begin{array}{l}\text { Chewing's } \\
\text { Fescue }\end{array}$ & 0 & 0 & 0 & 0 \\
\hline $\begin{array}{l}\text { Festuca } \\
\text { brevipila }\end{array}$ & $\begin{array}{l}\text { Hard Fescue } \\
\text { 'Chariot' }\end{array}$ & $\begin{array}{c}\text { Occurs in } \\
\text { WV }\end{array}$ & N/A & N/A & $N / A$ \\
\hline $\begin{array}{l}\text { Festuca } \\
\text { ovina var. } \\
\text { duriuscula }\end{array}$ & $\begin{array}{l}\text { Hard Fescue } \\
\text { 'Heron' }\end{array}$ & $\begin{array}{c}\text { Occurs in } \\
\text { WV }\end{array}$ & $\mathrm{N} / \mathrm{A}$ & $\mathrm{N} / \mathrm{A}$ & $N / A$ \\
\hline $\begin{array}{l}\text { Festuca } \\
\text { rubra }\end{array}$ & $\begin{array}{l}\text { Creeping Red } \\
\text { Fescue }\end{array}$ & 0 & 0 & 0 & 0 \\
\hline $\begin{array}{l}\text { Trifolium } \\
\text { repens }\end{array}$ & White Clover & 42 & 3 & 5 & 2 \\
\hline
\end{tabular}




\section{Developed Type B: Warm Season}

The Developed Type B mixture was created to control erosion on cut and fills with predominately native species. Common oat (Avena sativa L.), cereal rye (Secal cereal), big bluestem (Andropogon gerardi), virginia wildrye (Elymus virginicus ), autumn bentgrass (Agrostis perennans), switchgrass (Panicum virgatum), indiangrass (Sorghastrum nutan), partridge pea (Chamaecrista fasiculata), black-eyed susan (Rudbeckia hirta), narrowleaf mountainmint (Pycnanthemum tenuifolium), wild bergamot (Monarda fistulosa), panicledleaf ticktrefoil (Desmodium paniculatum), smooth oxeye (Heliopsis helianthoides), and flat-top goldentop (Euthamia graminifolia) were chosen to be in this mixture (Table 9). Species occurrence according to count by county, ecoregion, elevation, and watersheds are shown in Table 10.

Common oat and cereal rye are in this mixture for use as nurse crops. Oats will be used in spring plantings, while cereal rye will be substituted in and used in fall and winter plantings. Oat was specifically chosen as the spring nurse crop because the species grows in a large range of soil types and it winter kills. Due to winter killing, there is no threat that the nurse crop will continue to persist outside of one growing season. Cereal rye was chosen as the fall/winter nurse crop because the species is an annual graminoid and grows during cold climatic time. However, the species can become invasive and may need to be controlled based on site characteristics (Casey 2012). Both species are used as erosion control measures, and they aid in organic matter development (Ernst Conservation Seeds 2014).

Big bluestem is a warm season perennial bunchgrass that grows to a height of $6-8 \mathrm{ft}$ (1.83-2.44 m). The species has been used as an erosion control measure along roadways and for critical areas, but it may have invasive traits. This graminoid grows well in areas of low fertility, full or partial sun, and sandy and clay loam soils (Owsley 2011a). Virginia wildrye is a cool season perennial bunchgrass and grows to 2-3 ft (0.61-0.91 m). The species was chosen because it self-fertilizes, grows in moist and fine textured soils, and is shade tolerant (Shadow 
2009). Autumn bentgrass was chosen because the species grows to a height of $3.5 \mathrm{ft}(1.07 \mathrm{~m})$ in damp to dry soils and areas of full to partial sunlight. The species is also a native graminoid, but it does not withstand salt or drought (Ernst Conservation Seeds 2014). Switchgrass was selected for its erosion control characteristics, specifically for soil stabilization. The species grows to a height of 3-5 $\mathrm{ft}(0.91-1.52 \mathrm{~m})$ and prefers sandy to clay loam soils, deep soils, and dry to poorly drained sites. Switchgrass has also been found to grow well on shallow soils in the Eastern part of the United States (Jimmy Carter Plant Materials Center 2011). Indiangrass is presently utilized as an erosion control species along roadsides and critical area plantings. However, the species can become invasive in certain conditions. This graminoid grows to a height of 3-7 $\mathrm{ft}(0.91-2.13 \mathrm{~m})$, and is a warm season perennial bunchgrass. Indiangrass will grow in sandy to clay soils and poor to well drained soils. It prefers planting from May to June (Owsley 2011b).

Partridge pea is an annual legume that grows to a height of $1-3 \mathrm{ft}(0.30-0.91 \mathrm{~m})$ and has a yellow blooming flower. The legume is used for erosion control along roadways because it tolerates a large range of soils (Houck and Row 2006). Black-eyed susan is a biennial forb with yellow flowers that grows to a height of $3.3 \mathrm{ft}(1 \mathrm{~m})$. The species is vital to critical area plantings to aid in erosion control and development of the mixture (USDA- NRCS 2002c). Narrowleaf mountainmint is a warm season perennial forb that grows to a height of 2-3 ft (0.61-0.91 m) and develops white flowers. It is a soil stabilizing species that spreads quickly through rhizomes. This forb also is found growing in a wide range of environments (Sheahan 2012a). Wild bergamot is a flowering pink perennial forb, and it spreads through seed disbursement and rhizomes (Anderson 2003). This forb was chosen for beautification and ecological benefit for nectar seeking species.

Panicledleaf ticktrefoil is a perennial forb that grows to a height of $3 \mathrm{ft}(0.91 \mathrm{~m})$, has purple flowers, and fixates nitrogen to the soil (Kirk and Belt 2009). The nitrogen fixation ability of the species and the diversity it brings to the mixtures was an important contribution. Smooth 
oxeye is a perennial forb that grows to a height of 3-4 $\mathrm{ft}(0.91-1.22 \mathrm{~m})$ with yellow flowers. The species is found inhabiting roadsides and large ranges of soils. It aids in diversity and is a pollinator species (Taliga et al. 2012). Flat-top goldentop is a perennial forb that can grow up to 3-6 $\mathrm{ft}(0.91-1.83 \mathrm{~m})$ and is found along roadways and ditches. It has the potential to develop an invasive nature. The forb prefers moist soil conditions but tolerates poor soils and drought (Sheahan 2012b).

Table 9: Developed Type B-Warm Season; Planting time periods: Spring (March 1st - June 20th) and Fall (October 1st - November 1st)

\begin{tabular}{|c|c|c|c|c|c|}
\hline $\begin{array}{l}\text { Scientific } \\
\text { Name }\end{array}$ & Common Name & $\begin{array}{c}\text { Rate } \\
\text { (lb/ac) }\end{array}$ & $\begin{array}{l}\text { Cost } \\
\text { (/lb) }\end{array}$ & Classification & Description \\
\hline Avena sativa & $\begin{array}{l}\text { Common Oat } \\
\text { (Spring) }\end{array}$ & 30 & $\$ 0.26$ & Non-native & Nurse crop \\
\hline Secale cereale & Cereal Rye (Fall) & 30 & $\$ 0.34$ & Non-native & Nurse crop \\
\hline $\begin{array}{l}\text { Andropogon } \\
\text { gerardii }\end{array}$ & Big Bluestem & 6 & $\$ 10$ & Native & Graminoid \\
\hline $\begin{array}{l}\text { Elymus } \\
\text { virginicus }\end{array}$ & Virginia Wildrye & 8.4 & $\$ 8$ & Native & Graminoid \\
\hline $\begin{array}{l}\text { Agrostis } \\
\text { perennans }\end{array}$ & Autumn Bentgrass & 3.7 & $\$ 14$ & Native & Graminoid \\
\hline $\begin{array}{l}\text { Panicum } \\
\text { virgatum }\end{array}$ & Switchgrass & 6.4 & $\$ 5$ & Native & Graminoid \\
\hline $\begin{array}{l}\text { Sorghastrum } \\
\text { nutans }\end{array}$ & Indiangrass & 14 & $\$ 18$ & Native & Graminoid \\
\hline $\begin{array}{l}\text { Chamaecrista } \\
\text { fasciculata }\end{array}$ & Partridge Pea & 1 & $\$ 10$ & Native & Legume \\
\hline Rudbeckia hirta & Black-Eyed Susan & 0.6 & $\$ 24$ & Native & Forb \\
\hline $\begin{array}{l}\text { Pycnanthemum } \\
\text { tenuifolium }\end{array}$ & $\begin{array}{l}\text { Narrowleaf } \\
\text { Mountainmint }\end{array}$ & 0.1 & $\$ 80$ & Native & Forb \\
\hline $\begin{array}{l}\text { Monarda } \\
\text { fistulosa }\end{array}$ & Wild Bergamot & 0.5 & $\$ 96$ & Native & Forb \\
\hline $\begin{array}{l}\text { Desmodium } \\
\text { paniculatum }\end{array}$ & $\begin{array}{l}\text { Panicledleaf } \\
\text { Ticktrefoil }\end{array}$ & 0.4 & $\$ 48$ & Native & Forb \\
\hline $\begin{array}{l}\text { Heliopsis } \\
\text { helianthoides }\end{array}$ & Smooth Oxeye & 0.4 & $\$ 20$ & Native & Forb \\
\hline \multirow[t]{2}{*}{$\begin{array}{l}\text { Euthamia } \\
\text { graminifolia }\end{array}$} & Flat-top Goldentop & 0.1 & $\$ 400$ & Native & Forb \\
\hline & Total: & 71.6 & $\$ 8.64$ & $\$ 618.40 /$ acre & \\
\hline
\end{tabular}


Table 10: Warm Season Species Occurrence

\begin{tabular}{|c|c|c|c|c|c|}
\hline $\begin{array}{l}\text { Scientific } \\
\text { Name }\end{array}$ & Common Name & $\begin{array}{c}\text { Occurrence } \\
\text { (out of } 55 \\
\text { counties) }\end{array}$ & $\begin{array}{c}\text { Ecoregions } \\
\text { (out of } 3 \\
\text { regions) }\end{array}$ & $\begin{array}{c}\text { Elevation } \\
\text { (out of } 5 \\
\text { ranges) } \\
\end{array}$ & $\begin{array}{c}\text { Watershed } \\
\text { (out of } 2 \\
\text { watersheds) }\end{array}$ \\
\hline Avena sativa & $\begin{array}{l}\text { Common Oat } \\
\text { (Spring) }\end{array}$ & 6 & 3 & 5 & 1 \\
\hline Secale cereale & Cereal Rye (Fall) & 0 & 0 & 0 & 0 \\
\hline $\begin{array}{l}\text { Andropogon } \\
\text { gerardii }\end{array}$ & Big Bluestem & 23 & 3 & 5 & 2 \\
\hline $\begin{array}{l}\text { Elymus } \\
\text { virginicus }\end{array}$ & Virginia Wildrye & $\begin{array}{l}\text { Occurs in } \\
\text { WV }\end{array}$ & $\mathrm{N} / \mathrm{A}$ & $\mathrm{N} / \mathrm{A}$ & $\mathrm{N} / \mathrm{A}$ \\
\hline $\begin{array}{l}\text { Agrostis } \\
\text { perennans }\end{array}$ & $\begin{array}{l}\text { Autumn } \\
\text { Bentgrass }\end{array}$ & 32 & 3 & 5 & 2 \\
\hline $\begin{array}{l}\text { Panicum } \\
\text { virgatum }\end{array}$ & Switchgrass & 22 & 3 & 5 & 2 \\
\hline $\begin{array}{l}\text { Sorghastrum } \\
\text { nutans }\end{array}$ & Indiangrass & 26 & 3 & 5 & 2 \\
\hline $\begin{array}{l}\text { Chamaecrista } \\
\text { fasciculata }\end{array}$ & Partridge Pea & 13 & 3 & 5 & 2 \\
\hline Rudbeckia hirta & $\begin{array}{l}\text { Black-Eyed } \\
\text { Susan }\end{array}$ & 48 & 3 & 5 & 2 \\
\hline $\begin{array}{l}\text { Pycnanthemum } \\
\text { tenuifolium }\end{array}$ & $\begin{array}{l}\text { Narrowleaf } \\
\text { Mountainmint }\end{array}$ & $\begin{array}{l}\text { Occurs in } \\
\text { WV }\end{array}$ & N/A & $\mathrm{N} / \mathrm{A}$ & $\mathrm{N} / \mathrm{A}$ \\
\hline $\begin{array}{l}\text { Monarda } \\
\text { fistulosa }\end{array}$ & Wild Bergamot & 27 & 3 & 5 & 2 \\
\hline $\begin{array}{l}\text { Desmodium } \\
\text { paniculatum }\end{array}$ & $\begin{array}{l}\text { Panicledleaf } \\
\text { Ticktrefoil }\end{array}$ & 36 & 3 & 5 & 2 \\
\hline $\begin{array}{l}\text { Heliopsis } \\
\text { helianthoides }\end{array}$ & Smooth Oxeye & 30 & 3 & 5 & 2 \\
\hline $\begin{array}{l}\text { Euthamia } \\
\text { graminifolia }\end{array}$ & $\begin{array}{l}\text { Flat-top } \\
\text { Goldentop }\end{array}$ & 32 & 3 & 5 & 2 \\
\hline
\end{tabular}

\section{Developed Type C: Cool Season}

The Developed Type C mixture was intended for cool season plantings where a native mixture could not be used due to the planting time frame. The mixture can be planted on cut and fills. Erosion control, fast establishment, and visual appeal were the main traits considered. Redtop (Agrostis gigantea), chewing's fescue (Festuca rubra ssp. commutata), hard fescues 'Chariot' (Festuca brevipila) and 'Heron' (Festuca ovina var. duriuscula), creeping red fescue (Festuca rubra), white clover (Trifolium repens), and birdsfoot trefoil (Lotus corniculatus) were the chosen species (Table 11). Please see Table 12 for species occurrence in West Virginia. 
Redtop is a perennial rhizomatous graminoid with stems that grow to $2.5-3.3 \mathrm{ft}(0.76$ $1.00 \mathrm{~m})$. Presently, it is used for erosion control and in critical areas for stabilization. The species also grows in low fertility and acidic soils (USDA- NRCS 2002d). The hard fescue species are perennial cool season bunchgrasses that grow to a height of $2.5 \mathrm{ft}(0.76 \mathrm{~m})$. The species are frequently used for erosion control in recreational areas and lawns and along roadways. The graminoids are persistent and drought resistant with long life spans (Crowder N.D.). Creeping red fescue is a cool season bunch grass that has stems that grow to a height of $2 \mathrm{ft}(0.61 \mathrm{~m})$. This graminoid was chosen for its erosion control ability to bind the soil and stabilize a site. It is also frequently used in turf applications because it is resistant to wear, resilient, and tolerant of shade (USDA- NRCS 2002e).

White clover is a perennial legume with white flowering heads that may develop an invasive nature. The species was chosen to fixate nitrogen to the soil and act as an erosion control cover. It is presently used as an erosion control species (USDA- NRCS 2002f). Birdsfoot trefoil is a perennial legume with yellow flowers and a long lifespan. It grows to 2-3 $\mathrm{ft}$ (0.61-0.91 m). The legume was chosen for the species erosion control use along roadways, but it may develop an invasive nature (Bush 2002). The species was also chosen to fixate nitrogen to the soil to aid in the promotion of grass seedlings. 
Table 11: Developed Type C-Cool Season Seed Mixture; Planting time periods: Summer and Fall (August 1st - October 31st); Use an erosion control Birdsfoot Trefoil strand

\begin{tabular}{|c|c|c|c|c|c|c|}
\hline $\begin{array}{l}\text { Scientific } \\
\text { Name }\end{array}$ & $\begin{array}{l}\text { Common } \\
\text { Name }\end{array}$ & $\begin{array}{l}\text { Rate } \\
\text { (Ib/ac) }\end{array}$ & $\begin{array}{l}\text { Cost } \\
\text { (/lb) }\end{array}$ & Classification & Description & $\begin{array}{l}\text { Threat } \\
\text { Level }\end{array}$ \\
\hline $\begin{array}{l}\text { Agrostis } \\
\text { gigantea }\end{array}$ & Redtop & 4 & $\$ 10$ & Non-Native & Nurse crop & 3 \\
\hline $\begin{array}{l}\text { Festuca } \\
\text { ovina var. } \\
\text { duriuscula }\end{array}$ & $\begin{array}{l}\text { Hard Fescue } \\
\text { 'Heron' }\end{array}$ & 10 & $\$ 2.80$ & Non-Native & Graminoid & Introduced \\
\hline $\begin{array}{l}\text { Festuca } \\
\text { brevipila }\end{array}$ & $\begin{array}{l}\text { Hard Fescue } \\
\text { 'Chariot' }\end{array}$ & 10 & $\$ 2.80$ & Non-Native & Graminoid & Introduced \\
\hline $\begin{array}{l}\text { Festuca } \\
\text { rubra }\end{array}$ & $\begin{array}{l}\text { Creeping Red } \\
\text { Fescue }\end{array}$ & 16 & $\$ 1.50$ & Non-Native & Graminoid & Introduced \\
\hline $\begin{array}{l}\text { Trifolium } \\
\text { repens }\end{array}$ & White Clover & 7 & $\$ 3.80$ & Non-Native & Legume & 3 \\
\hline $\begin{array}{l}\text { Lotus } \\
\text { corniculatus }\end{array}$ & $\begin{array}{l}\text { Birdsfoot } \\
\text { Trefoil }\end{array}$ & 8 & $\$ 5.25$ & Non-Native & Legume & 3 \\
\hline & Total: & 55 & $\$ 3.43$ & $\$ 188.60$ /acre & & \\
\hline
\end{tabular}

Table 12: Cool Season Species Occurrence

\begin{tabular}{|c|c|c|c|c|c|}
\hline $\begin{array}{l}\text { Scientific } \\
\text { Name }\end{array}$ & Common Name & $\begin{array}{c}\text { Occurrence } \\
\text { (out of } 55 \\
\text { counties) }\end{array}$ & $\begin{array}{c}\text { Ecoregions } \\
\text { (out of } 3 \\
\text { regions) } \\
\end{array}$ & $\begin{array}{l}\text { Elevation } \\
\text { (out of } 5 \\
\text { ranges) } \\
\end{array}$ & $\begin{array}{c}\text { Watershed } \\
\text { (out of } 2 \\
\text { watersheds) }\end{array}$ \\
\hline $\begin{array}{l}\text { Agrostis } \\
\text { gigantea }\end{array}$ & Red Top & 46 & 3 & 5 & 2 \\
\hline $\begin{array}{l}\text { Festuca } \\
\text { ovina var. } \\
\text { duriuscula }\end{array}$ & $\begin{array}{l}\text { Hard Fescue } \\
\text { 'Heron' }\end{array}$ & $\begin{array}{l}\text { Occurs in* } \\
\text { WV }\end{array}$ & $N / A$ & $\mathrm{~N} / \mathrm{A}$ & $\mathrm{N} / \mathrm{A}$ \\
\hline $\begin{array}{l}\text { Festuca } \\
\text { brevipila }\end{array}$ & $\begin{array}{l}\text { Hard Fescue } \\
\text { 'Chariot' }\end{array}$ & $\begin{array}{l}\text { Occurs in* } \\
\text { WV }\end{array}$ & $N / A$ & $\mathrm{~N} / \mathrm{A}$ & $\mathrm{N} / \mathrm{A}$ \\
\hline $\begin{array}{l}\text { Festuca } \\
\text { rubra }\end{array}$ & $\begin{array}{l}\text { Creeping Red } \\
\text { Fescue }\end{array}$ & 0 & 0 & 0 & 0 \\
\hline $\begin{array}{l}\text { Trifolium } \\
\text { repens }\end{array}$ & White Clover & 42 & 3 & 5 & 2 \\
\hline $\begin{array}{l}\text { Lotus } \\
\text { corniculatus }\end{array}$ & Birdsfoot Trefoil & 10 & 3 & 5 & 1 \\
\hline
\end{tabular}

\section{Developed Type D: High Elevation}

Developed Type D mixture was developed for planting in the high elevation regions within West Virginia. The elevation specified is any location above 2,400 ft $(731.5 \mathrm{~m})$.

Developed Type D has two sub-mixtures classified as Developed Type D1 and Developed Type D2. The mixtures vary only by seeding rate based on slope conditions. Developed Type D1 is 
for slopes $\leq 3: 1(\mathrm{H}: \mathrm{V})$ and requires a lower seeding rate due to the reduced erosion risk (Table 13). Developed Type D2 is for slopes $>3: 1(\mathrm{H}: \mathrm{V})$ and has a higher seeding rate due to the increased erosion risk for vegetation on steeper slopes (Table 14).

This mixture was developed on the basis of using native species since the non-native mixtures presently used by the WVDOH have not been effective. The native species were chosen based on their occurrence in the high elevation regions of the state. Common oat (Avena sativa), cereal rye (Secal cereale), big bluestem (Andropogon gerardii), virginia wildrye (Elymus virginicus), autumn bentgrass (Agrostis perennans), deertongue (Dichanthelium clandestinum), little bluestem (Schizachyrium scoparium), partridge pea (Chamaecrista fasiculata), black-eyed susan (Rudbeckia hirta), smooth oxeye (Heliopsis helianthoides), wrinkleleaf goldenrod (Solidago rugose), narrowleaf mountainmint (Pycnanthemum tenuifolium), panicledleaf ticktrefoil (Desmodium paniculatum), and flat-top goldentop (Euthamia graminifolia) were chosen to be in this mixture (Table 13 and 14). Please see Table 15 for species occurrence.

Common oat and cereal rye are in this mixture for the use as nurse crops. Oats will be used in spring plantings, while cereal rye will be substituted in and used in fall/winter plantings. Oat was specifically chosen as the spring nurse crop for its ability to grow in a large range of soil types and it winter kills. Due to winter kill, there is no threat that the nurse crop will continue to persist. Cereal rye was chosen as the fall/winter nurse crop because it is an annual graminoid and grows during cold climatic times. However, the species can become invasive and may need to be controlled based on site characteristics (Casey 2012). Both species are used as erosion control measures, and they aid in organic matter development (Ernst Conservation Seeds 2014).

Big bluestem is a warm season perennial bunchgrass that grows to a height of $6-8 \mathrm{ft}$ (1.83-2.44 m) and may experience invasive traits. The species has been used as an erosion control measure along roadways and for critical areas. This graminoid grows well in areas of low 
fertility, full or partial sun, and sandy and clay loam soils (Owsley 2011a). Virginia wildrye is a cool season perennial bunchgrass and grows to 2-3 ft $(0.61-0.91 \mathrm{~m})$. The species was chosen because it self-fertilizes, grows in moist and fine textured soils, and is shade tolerant (Shadow 2009). Autumn bentgrass was selected because it grows to a height of $3.5 \mathrm{ft}(1.07 \mathrm{~m})$ in damp to dry soils and areas of full to partial sunlight. The species is also a native graminoid, but does not withstand salt or drought (Ernst Conservation Seeds 2014). Deertongue is a warm season perennial graminoid that grows to a height of $1-3 \mathrm{ft}(0.30-0.91 \mathrm{~m})$. The species was chosen for its ability to tolerate high levels of aluminum and to grow in acidic and low fertility soils. This graminoid prefers to grow in coarse textured soils and creates a mat of vegetation to cover and protect the ground during winter. The species is currently used in revegetating mine locations and other disrupted landscapes (USDA- NRCS 2002a). Little bluestem is a warm season graminoid that grows to a height of $1-3 \mathrm{ft}(0.30-0.91 \mathrm{~m})$. The species was chosen because it is drought tolerant, useful for erosion control, grows in a wide range of soils, and occurs frequently in West Virginia (USDA- NRCS 2002b).

Partridge pea is an annual legume with a yellow blooming flower that grows to a height of $1-3 \mathrm{ft}(0.30-0.91 \mathrm{~m})$. It is used for erosion control along roadways because it tolerates a large range of soils (Houck and Row 2006). Black-eyed susan is a biennial forb that grows to a height of $3.3 \mathrm{ft}(1 \mathrm{~m})$ and has yellow flowers. The species is vital to critical area plantings to aid in erosion control and development of the mixture (USDA- NRCS 2002c). Smooth oxeye is a yellow-flowered perennial forb that grows to a height of 3-4 $\mathrm{ft}(0.91-1.22 \mathrm{~m})$. The species is found inhabiting roadsides and large ranges of soils. It aids in diversity and is a pollinator species (Taliga et al. 2012). Wrinkleleaf goldenrod is a forb that can be found in fields and along roadsides and woods. The forb can grow to a height of 1-3.5 ft (0.30-1.07 m), is not tolerant of salt, and has yellow blooming flowers. The goldenrod withstands shade and some drought (Ernst Conservation Seeds 2014). 
Narrowleaf mountainmint is a warm season perennial forb that grows to a height of 2-3 ft (0.61-0.91 $\mathrm{m})$ and develops white flowers. It is a soil stabilizing species that spreads quickly through rhizomes. This forb also is found growing in a wide range of environments (Sheahan 2012a). Panicledleaf ticktrefoil is a perennial forb that grows to a height of $3 \mathrm{ft}(0.91 \mathrm{~m})$, has purple flowers, and fixates nitrogen to the soil (Kirk and Belt 2009). The nitrogen fixation ability and the diversity it brings to the mixtures was an important contribution. Flat-top goldentop is a perennial forb that can grow up to $3-6 \mathrm{ft}(0.91-1.83 \mathrm{~m})$ that could develop an invasive nature. The forb is found along roadways and ditches. It prefers moist soil conditions but tolerates poor soils and drought (Sheahan 2012b). 
Table 13: Developed Type D1-High Elevation Seed Mixture; Planting time periods: Spring (March 1st - June 20th) and Fall (October 1st - November 1st); For Slopes $\leq 3: 1(\mathrm{H:V})$

\begin{tabular}{|c|c|c|c|c|c|}
\hline Scientific Name & Common Name & $\begin{array}{l}\text { Rate } \\
\text { (lb/ac) }\end{array}$ & $\begin{array}{l}\text { Cost } \\
\text { (/lb) }\end{array}$ & Classification & Description \\
\hline Avena sativa & $\begin{array}{l}\text { Common Oat } \\
\text { (Spring) }\end{array}$ & 30 & $\$ 0.26$ & Non-native & Nurse crop \\
\hline Secale cereale & Cereal Rye (Fall) & 30 & $\$ 0.34$ & Non-native & Nurse crop \\
\hline Andropogon gerardii & Big Bluestem & 2.5 & $\$ 10$ & Native & Graminoid \\
\hline Elymus virginicus & Virginia Wildrye & 4 & $\$ 8$ & Native & Graminoid \\
\hline Agrostis perennans & Autumn Bentgrass & 2.7 & $\$ 14$ & Native & Graminoid \\
\hline $\begin{array}{l}\text { Dichanthelium } \\
\text { clandestinum }\end{array}$ & Deertongue & 2.0 & $\$ 18$ & Native & Graminoid \\
\hline $\begin{array}{l}\text { Schizachyrium } \\
\text { scoparium }\end{array}$ & Little Bluestem & 6.5 & $\$ 24$ & Native & Graminoid \\
\hline $\begin{array}{l}\text { Chamaecrista } \\
\text { fasciculata }\end{array}$ & Partridge Pea & 0.8 & $\$ 10$ & Native & Legume \\
\hline Rudbeckia hirta & Black-Eyed Susan & 0.6 & $\$ 24$ & Native & Forb \\
\hline $\begin{array}{l}\text { Heliopsis } \\
\text { helianthoides }\end{array}$ & Smooth Oxeye & 0.4 & $\$ 20$ & Native & Forb \\
\hline Solidago rugosa & $\begin{array}{l}\text { Wrinkleleaf } \\
\text { Goldenrod }\end{array}$ & 0.2 & $\$ 280$ & Native & Forb \\
\hline $\begin{array}{l}\text { Pycnanthemum } \\
\text { tenuifolium }\end{array}$ & $\begin{array}{l}\text { Narrowleaf } \\
\text { Mountainmint }\end{array}$ & 0.1 & $\$ 80$ & Native & Forb \\
\hline $\begin{array}{l}\text { Desmodium } \\
\text { paniculatum }\end{array}$ & $\begin{array}{l}\text { Panicledleaf } \\
\text { Ticktrefoil }\end{array}$ & 0.4 & $\$ 48$ & Native & Forb \\
\hline \multirow[t]{2}{*}{$\begin{array}{l}\text { Euthamia } \\
\text { graminifolia }\end{array}$} & Flat-top Goldentop & 0.1 & $\$ 400$ & Native & Forb \\
\hline & Total: & 50.3 & $\$ 8.91$ & $\$ 448.20 /$ acre & \\
\hline
\end{tabular}


Table 14: Developed Type D2-High Elevation Seed Mixture; Planting time periods: Spring (March 1st - June 20th) and Fall (October 1st - November 1st); For Slopes >3:1

\begin{tabular}{|c|c|c|c|c|c|}
\hline Scientific Name & Common Name & $\begin{array}{c}\text { Rate } \\
\text { (Ib/ac) }\end{array}$ & $\begin{array}{l}\text { Cost } \\
\text { (/lb) }\end{array}$ & Classification & Description \\
\hline Avena sativa & $\begin{array}{l}\text { Common Oat } \\
\text { (Spring) }\end{array}$ & 30 & $\$ 0.26$ & Non-native & Nurse crop \\
\hline Secale cereale & Cereal Rye (Fall) & 30 & $\$ 0.34$ & Non-native & Nurse crop \\
\hline Andropogon gerardii & Big Bluestem & 4 & $\$ 10$ & Native & Graminoid \\
\hline Elymus virginicus & Virginia Wildrye & 8.2 & $\$ 8$ & Native & Graminoid \\
\hline Agrostis perennans & Autumn Bentgrass & 5.5 & $\$ 14$ & Native & Graminoid \\
\hline $\begin{array}{l}\text { Dichanthelium } \\
\text { clandestinum }\end{array}$ & Deertongue & 8 & $\$ 18$ & Native & Graminoid \\
\hline $\begin{array}{l}\text { Schizachyrium } \\
\text { scoparium }\end{array}$ & Little Bluestem & 15 & $\$ 24$ & Native & Graminoid \\
\hline $\begin{array}{l}\text { Chamaecrista } \\
\text { fasciculata }\end{array}$ & Partridge Pea & 0.8 & $\$ 10$ & Native & Legume \\
\hline Rudbeckia hirta & Black-Eyed Susan & 0.6 & $\$ 24$ & Native & Forb \\
\hline $\begin{array}{l}\text { Heliopsis } \\
\text { helianthoides }\end{array}$ & Smooth Oxeye & 0.4 & $\$ 20$ & Native & Forb \\
\hline Solidago rugosa & $\begin{array}{l}\text { Wrinkleleaf } \\
\text { Goldenrod }\end{array}$ & 0.2 & $\$ 280$ & Native & Forb \\
\hline $\begin{array}{l}\text { Pycnanthemum } \\
\text { tenuifolium }\end{array}$ & $\begin{array}{l}\text { Narrowleaf } \\
\text { Mountainmint }\end{array}$ & 0.1 & $\$ 80$ & Native & Forb \\
\hline $\begin{array}{l}\text { Desmodium } \\
\text { paniculatum }\end{array}$ & $\begin{array}{l}\text { Panicledleaf } \\
\text { Ticktrefoil }\end{array}$ & 0.4 & $\$ 48$ & Native & Forb \\
\hline \multirow[t]{2}{*}{$\begin{array}{l}\text { Euthamia } \\
\text { graminifolia }\end{array}$} & $\begin{array}{l}\text { Flat-top } \\
\text { Goldentop }\end{array}$ & 0.1 & $\$ 400$ & Native & Forb \\
\hline & Total: & 73.3 & $\$ 11.57$ & $\$ 848.00 /$ acre & \\
\hline
\end{tabular}


Table 15: High Elevation Species Occurrence

\begin{tabular}{|c|c|c|c|c|c|}
\hline $\begin{array}{l}\text { Scientific } \\
\text { Name }\end{array}$ & $\begin{array}{l}\text { Common } \\
\text { Name }\end{array}$ & $\begin{array}{c}\text { Occurrence } \\
\text { (out of } 55 \\
\text { counties) }\end{array}$ & $\begin{array}{c}\text { Ecoregions } \\
\text { (out of } 3 \\
\text { regions) }\end{array}$ & $\begin{array}{l}\text { Elevation } \\
\text { (out of } 5 \\
\text { ranges) } \\
\end{array}$ & $\begin{array}{c}\text { Watershed } \\
\text { (out of } 2 \\
\text { watersheds) }\end{array}$ \\
\hline Avena sativa & $\begin{array}{l}\text { Common Oat } \\
\text { (Spring) }\end{array}$ & 6 & 3 & 5 & 1 \\
\hline Secale cereale & $\begin{array}{l}\text { Cereal Rye } \\
\text { (Fall) }\end{array}$ & 0 & 0 & 0 & 0 \\
\hline $\begin{array}{l}\text { Andropogon } \\
\text { gerardii }\end{array}$ & Big Bluestem & 23 & 3 & 5 & 2 \\
\hline $\begin{array}{l}\text { Elymus } \\
\text { virginicus }\end{array}$ & Virginia Wildrye & $\begin{array}{c}\text { Occurs in } \\
\text { WV }\end{array}$ & $N / A$ & $\mathrm{~N} / \mathrm{A}$ & $\mathrm{N} / \mathrm{A}$ \\
\hline $\begin{array}{l}\text { Agrostis } \\
\text { perennans }\end{array}$ & $\begin{array}{l}\text { Autumn } \\
\text { Bentgrass }\end{array}$ & 32 & 3 & 5 & 2 \\
\hline $\begin{array}{l}\text { Dichanthelium } \\
\text { clandestinum }\end{array}$ & Deertongue & 39 & 3 & 5 & 2 \\
\hline $\begin{array}{l}\text { Schizachyrium } \\
\text { scoparium }\end{array}$ & Little Bluestem & 23 & 3 & 5 & 2 \\
\hline $\begin{array}{l}\text { Chamaecrista } \\
\text { fasciculata }\end{array}$ & Partridge Pea & 13 & 3 & 5 & 2 \\
\hline Rudbeckia hirta & $\begin{array}{l}\text { Black-Eyed } \\
\text { Susan }\end{array}$ & 48 & 3 & 5 & 2 \\
\hline $\begin{array}{l}\text { Heliopsis } \\
\text { helianthoides }\end{array}$ & Smooth Oxeye & 30 & 3 & 5 & 2 \\
\hline $\begin{array}{l}\text { Solidago } \\
\text { rugosa }\end{array}$ & $\begin{array}{l}\text { Wrinkleleaf } \\
\text { Goldenrod }\end{array}$ & 23 & 3 & 5 & 2 \\
\hline $\begin{array}{l}\text { Pycnanthemum } \\
\text { tenuifolium }\end{array}$ & $\begin{array}{l}\text { Narrowleaf } \\
\text { Mountainmint }\end{array}$ & $\begin{array}{l}\text { Occurs in } \\
\text { WV }\end{array}$ & $\mathrm{N} / \mathrm{A}$ & $\mathrm{N} / \mathrm{A}$ & $\mathrm{N} / \mathrm{A}$ \\
\hline $\begin{array}{l}\text { Desmodium } \\
\text { paniculatum }\end{array}$ & $\begin{array}{l}\text { Panicledleaf } \\
\text { Ticktrefoil }\end{array}$ & 36 & 3 & 5 & 2 \\
\hline $\begin{array}{l}\text { Euthamia } \\
\text { graminifolia }\end{array}$ & $\begin{array}{l}\text { Flat-top } \\
\text { Goldentop }\end{array}$ & 32 & 3 & 5 & 2 \\
\hline
\end{tabular}

\section{Developed Type E: Wet Areas}

Developed Type E mixture was created to be used in areas that experience constant standing water or moist conditions. The mixture contains fox sedge (Carex vulpinoidea), fowl bluegrass (Poa palustris), redtop panicgrass (Panicum rigidulum), common rush (Juncus effusus), virginia wildrye (Elymus virginicus), shallow sedge (Carex lurida), blue vervain (Verbena hastata), and wingstem (Verbesina alternifolia) seen in Table 16. Species occurrence can be seen in Table 17. 
Fox sedge spreads quickly, grows in ditches, and is a pioneer wetland colonizer. This graminoid is a bunch-forming perennial that can reach a height of $3.9 \mathrm{ft}(1.2 \mathrm{~m})$ and has been found growing with virginia wildrye and other sedges (Wennerberg 2006). Fowl bluegrass was selected because it develops quickly and is found in moist soils. This graminoid is a bunchforming cool season species that reaches a height of $4 \mathrm{ft}(1.22 \mathrm{~m})$ (Ernst Conservation Seeds 2014). Redtop panicgrass was chosen because it is a common wetland species. This graminoid develops bunches and can grow to a height of $4 \mathrm{ft}(1.22 \mathrm{~m})$. It does not tolerate salt but can survive in full sunlight and fire (Ernst Conservation Seeds 2014). Common rush was selected because it has a deep root system, inhabits ditches and wet meadows, and withstands a variety of site characteristics. This bunch-forming species is a perennial that grows up to $4 \mathrm{ft}(1.22 \mathrm{~m})$ (Stevens 2003).

Virginia wildrye is a cool season perennial bunchgrass that grows to $2-3 \mathrm{ft}(0.61-0.91 \mathrm{~m})$. The species was chosen because it self-fertilizes, grows in moist and fine textured soils, and is shade tolerant (Shadow 2009). Shallow sedge is a bunch-forming species that can reach a height of $3 \mathrm{ft}(0.91 \mathrm{~m})$. It does not withstand salt or drought but can tolerate some shade (Ernst Conservation Seeds 2014). Blue vervain was chosen as part of the mixture because it can grow in disturbed areas. The perennial forb can grow to a height of 2-5 ft (0.61-1.52 $\mathrm{m})$ and has purple flowers. It also spreads through rhizomes and prefers to grow in sites with moist soils and full to partial sunlight (Kirk and S.Belt 2010). Wingstem was an important component due to its occurrence along roadsides, vigorous growth, and preference for moist soils. This forb develops yellow flowers and grows to 3-9 ft (0.91-2.74 m) (Ernst Conservation Seeds 2014). 
Table 16: Developed Type E-Wet Areas Seed Mixture; Planting time periods: Spring (March 20th - June 30th) and Fall (October 15th- Good planting conditions end)

\begin{tabular}{|c|c|c|c|c|c|}
\hline Scientific Name & Common Name & $\begin{array}{c}\text { Rate } \\
\text { (lb/ac) }\end{array}$ & $\begin{array}{l}\text { Cost } \\
\text { (/lb) }\end{array}$ & Classification & Description \\
\hline Carex vulpinoidea* & Fox Sedge & 4 & $\$ 24$ & Native & Graminoid \\
\hline Poa palustris & Fowl Bluegrass & 3.5 & $\$ 10$ & Native & Graminoid \\
\hline Panicum rigidulum & $\begin{array}{l}\text { Redtop } \\
\text { Panicgrass }\end{array}$ & 4 & $\$ 48$ & Native & Graminoid \\
\hline Juncus effusus & Common Rush & 0.5 & $\$ 66$ & Native & Graminoid \\
\hline Elymus virginicus & Virginia Wildrye & 4 & $\$ 8$ & Native & Graminoid \\
\hline Carex lurida & Shallow Sedge & 3 & $\$ 64$ & Native & Graminoid \\
\hline Verbena hastata & Blue Vervain & 0.8 & $\$ 40$ & Native & Forb \\
\hline \multirow[t]{2}{*}{$\begin{array}{l}\text { Verbesina } \\
\text { alternifolia }\end{array}$} & Wingstem & 0.2 & $\$ 180$ & Native & Forb \\
\hline & Total: & 20 & $\$ 32.40$ & $\$ 648.00 /$ acre & \\
\hline
\end{tabular}

${ }^{\star}$ Good planting conditions require direct mud and seed contact. Seed will stay dormant in fall planting. (For entire mixture)

Table 17: Wet Area Species Occurrence

\begin{tabular}{|c|c|c|c|c|c|}
\hline $\begin{array}{l}\text { Scientific } \\
\text { Name }\end{array}$ & Common Name & $\begin{array}{c}\text { Occurrence } \\
\text { (out of } 55 \\
\text { counties) }\end{array}$ & $\begin{array}{c}\text { Ecoregions } \\
\text { (out of } 3 \\
\text { regions) }\end{array}$ & $\begin{array}{c}\text { Elevation } \\
\text { (out of } 5 \\
\text { ranges) }\end{array}$ & $\begin{array}{c}\text { Watershed } \\
\text { (out of } 2 \\
\text { watersheds) }\end{array}$ \\
\hline $\begin{array}{l}\text { Carex } \\
\text { vulpinoidea }\end{array}$ & Fox Sedge & 41 & 3 & 5 & 2 \\
\hline $\begin{array}{l}\text { Poa } \\
\text { palustris }\end{array}$ & Fowl Bluegrass & 11 & 3 & 5 & 2 \\
\hline $\begin{array}{l}\text { Panicum } \\
\text { rigidulum }\end{array}$ & $\begin{array}{l}\text { Redtop } \\
\text { Panicgrass }\end{array}$ & 21 & 3 & 5 & 2 \\
\hline $\begin{array}{l}\text { Juncus } \\
\text { effusus }\end{array}$ & Common Rush & 42 & 3 & 5 & 2 \\
\hline $\begin{array}{l}\text { Elymus } \\
\text { virginicus }\end{array}$ & Virginia Wildrye & $\begin{array}{c}\text { Occurs in } \\
\text { WV }\end{array}$ & $\mathrm{N} / \mathrm{A}$ & N/A & $\mathrm{N} / \mathrm{A}$ \\
\hline $\begin{array}{l}\text { Carex } \\
\text { lurida }\end{array}$ & Shallow Sedge & 48 & 3 & 5 & 2 \\
\hline $\begin{array}{l}\text { Verbena } \\
\text { hastata }\end{array}$ & Blue Vervain & 29 & 3 & 5 & 2 \\
\hline $\begin{array}{l}\text { Verbesina } \\
\text { alternifolia }\end{array}$ & Wingstem & 36 & 3 & 5 & 2 \\
\hline
\end{tabular}

\subsection{Discussion}

The developed seed mixtures were compared and contrasted to the current mixtures used or approved by the WVDOH and WVDEP. Both agencies focus on permanent seeding of disturbed areas to control the effects of erosion and to stabilize project sites. The developed 
seed mixtures contain various similarities and differences compared to the WVDOH and WVDEP seed mixtures.

The WVDOH has four main permanent seed mixtures. Type B is a mixture for medians, shoulders, and mowable areas. Type $C$ is composed of two sub-mixtures. Type $C-1$ is for coarse lawns, while Type C-2 is for fine lawns. Type D is used for cut and fills slopes. Finally, Type $L$ is used for all areas. The WVDOH primarily uses Type B and D seed mixtures (WVDOH 2010).

Developed Type A and WVDOH Type B are both intended for medians, shoulders, and mowable areas. However, Developed Type A was also designed to be used for lawns, which eliminates the WVDOH Type C mixtures. Due to the WVDOH mowing frequency and cutting height, a combined mixture was determined to be most appropriate because mowed areas were being cut to lawn heights. The WVDOH Type B contains five species. One of the graminoids, Kentucky 31 fescue, is a threat level 1 species and was purposely excluded from the proposed mixture (WVDNR Natural Heritage Program 2009). All threat level 1 and 2 species were avoided due to concern about the negative ecological impact of those species on West Virginia. Annual ryegrass or weeping lovegrass are used as cover crops in the WVDOH Type B mixture. The current cover crops were not considered for the develped mixture because of their undesirable persistence characteristics. Developed Type A also contains autumn bentgrass, chewing's fescue, and two strands of hard fescue. The additional species were chosen for characteristics, detailed in Section 3.3.2, that would create an appropriate mowable area mixture. The two mixtures also differ in seeding rates. The WVDOH Type B has a total of $95 \mathrm{lb} / \mathrm{ac}(106 \mathrm{~kg} / \mathrm{ha})$ of seed and was priced at \$150/acre (WVDOH 2010). Where Developed Type A has $163 \mathrm{lb} / \mathrm{ac}$ (183 kg/ha) and was priced at $\$ 482 /$ acre. The difference comes from the fact that Developed Type $A$ is being used for all mowable areas and is being treated as a lawn mixture. Lawn mixtures have higher seeding rates to create a densely vegetated mat that is visibly appealing to users. 
WVDOH Types C-1 and C-2 utilize red fescue, which was also used in the Developed Type A mixture. Type C-1 includes Kentucky 31 fescue, which is a threat level 1 species, and Kentucky bluegrass, which is a threat level 2 species (WVDNR Natural Heritage Program 2009). Type C-2 uses Kentucky bluegrass (Poa pratensis L.) and merion bluegrass (strand of Kentucky bluegrass), both threat level 2 species (WVDNR Natural Heritage Program 2009). Developed Type A purposely avoided these high threat level species and also did not use a cover crop like annual ryegrass or weeping lovegrass (WVDOH 2010). WVDOH Types C-1 and C-2 cost $\$ 182 /$ acre and $\$ 241 /$ acre and have a total seeding rate of $93 \mathrm{lb} /$ acre and $97 \mathrm{lb} /$ acre. In contrast, Developed Type A cost $\$ 482 /$ acre and has a seeding rate of $163 \mathrm{lb} /$ acre.

WVDOH Type D and Developed Type B are both intended for cut and fill slopes. Due to the WVDNR concern about invasive and non-native species, Developed Type B was developed to be predominantly a native seed mixture. The only non-native species were the two cover crops. None of the WVDOH Type D species were chosen because they are non-native species. Crownvetch and Kentucky 31 fescue especially were not selected because they are threat level 1 species (WVDNR Natural Heritage Program 2009). WVDOH Type D only contains 5 species and has a low diversity as a mixture. Developed Type B has 14 species consisting of various graminoids, nurse crops, forbs, and a legume. The diversity of the mixture was important to fulfill the different microsites that may occur over a project site. WVDOH Type D does not contain any forbs, which are an important contribution to aid in long term vegetative cover and erosion control. WVDOH Type D cost \$684/acre where Developed Type B cost \$618/acre and contains native species (WVDOH 2010).

WVDOH Type $L$ is a mixture that is not commonly used by the WVDOH. It only has four graminoids and lacks diversity. There is no developed mixture with which to compare Type L. The WVDOH was determined to be lacking mixtures for wet areas and high elevation. The deficiency was first noted by the Natural Heritage Vegetation Ecologist (Elizabeth A. Byers, personal communication, October 8th- December 5th, 2014), and Developed Types D and 
Developed Type E were created. The mixtures will likely be used infrequently, but they are important to control erosion in wet areas and areas of high elevation. A high elevation mixture was recommended by the WVDNR and WVDOH because field experience indicated present mixtures were not establishing appropriately. The developed high elevation mixture incorporates native species that are found growing in the high elevations of West Virginia. Its efficiency was tested in the field (WVDOH 2010).

The WVDEP (2006) has 29 seed mixtures for permanent seeding. Types C and D are designated as fine and coarse lawn mixtures. These mixtures also do not contain a cover crop, like Developed Type A. The WVDEP (2006) specifically states that Types C and D are for use in urban areas. Developed Type A can be used in urban and non-urban areas because the areas of planting will be maintained similar to urban locations with frequent mowing. Developed Type A contains red fescue and white clover like WVDEP Types $C$ and D, but does not include Kentucky 31 fescue (threat level 1 species), Kentucky bluegrass (threat level 2 species), or merion bluegrass (threat level 2 species) (WVDNR Natural Heritage Program 2009). Developed Type A has a seeding rate of $163 \mathrm{lb} / \mathrm{ac}(183 \mathrm{~kg} / \mathrm{ha})$, while WVDEP Types C and D have seeding rates of $90 \mathrm{lb} / \mathrm{ac}(101 \mathrm{~kg} / \mathrm{ha})$ and $95 \mathrm{lb} / \mathrm{ac}(106 \mathrm{~kg} / \mathrm{ha})$, respectively. The difference in seeding rate is due to the fact that WVDEP Types $C$ and $D$ are being used strictly in urban settings that are typically gentle slopes. Developed Type A will be used along roadways throughout all of West Virginia, which will include a wide range of slopes that require a higher seeding rate. The higher seeding rate will accommodate the diverse conditions to provide an adequate number of successful seedlings (WVDEP 2006).

WVDEP Types A-2 and A-3 are native mixtures and are comparable to Developed Type B and Developed Type D. Developed Type B and Developed Type D are native mixtures intended for cut and fills and high elevations, respectively. The WVDEP does not designate the specific use of their mixtures, except to state that Types $C$ and D are for lawns. However, Types A-2 and A-3 could be used for cut and fills, high elevations, and erosion control. The WVDEP 
mixtures have a seeding rate of $35 \mathrm{lb} / \mathrm{ac}(39 \mathrm{~kg} / \mathrm{ha})$, which seems to be a low seeding rate compared to $71.6 \mathrm{lb} / \mathrm{ac}(80.3 \mathrm{~kg} / \mathrm{ha})$ and $73.3 \mathrm{lb} / \mathrm{ac}(82.2 \mathrm{~kg} / \mathrm{ha})$ for Developed Types B and Developed Type D. The WVDEP native mixtures could be appropriate on slopes that are not steep in nature. The Developed Type B and Developed Type D have higher seeding rates because they will be on moderate to steep slopes, planted on poor soils, and likely mixed in a hydromulch. The developed mixtures include all the species of Types A-2 and A-3 except eastern gamagrass and sideoats grama. The developed mixtures do not include these species because they do not frequently occur in West Virginia (USDA, NRCS 2015). The developed mixtures are also diverse mixtures that include a cover crop, graminoids, a legume, and forbs. The diversity will enable the mixtures to inhabit multiple microsites over a project site. The WVDEP native mixtures are weak in diversity and only include graminoids. The inclusion of a cover crop, a legume, and forbs would allow those mixtures to vegetate and secure a project location (WVDEP 2006).

WVDEP Type S mixture can be compared to the Developed Type E wet area mixture. Type S only includes reed canarygrass and weeping lovegrass. The weeping lovegrass is a non-native being used as a covercrop and erosion control measure (USDA-NRCS 2002g). The species does not like wet areas, although reed canarygrass does. Reed canarygrass is a species that can be used for erosion control in wet areas and can even be used in a planting scenario to aid in filtering runoff (USDA-NRCS 2002h). However, reed canarygrass is a threat level 1 species and should be avoided (WVDNR Natural Heritage Program 2009). Type S is the only mixture that could be used for wet areas, but the WVDEP does not specify the use. Developed Type $\mathrm{E}$ is a native mixture that has both graminoids and forbs, while Type $\mathrm{S}$ only has graminoids. The diversity aids in site stabilization and long term development for wet areas (WVDEP 2006).

Any WVDEP seed mixtures not mentioned above can be closely compared to Developed Type C. The WVDEP does specify that some of these mixtures can be used for low 
maintenance, no maintenance, and pasture areas. The Developed Type C mixture is intended to be used on cut and fills in the fall planting season. The jointly used species are red fescue, birdsfoot trefoil, redtop, and white clover. These species are of low invasive nature (WVDNR Natural Heritage Program 2009) and are commonly used for erosion control and site stabilization (Bush 2002, USDA-NRCS 2002d, and USDA-NRCS 2002f). None of the WVDEP mixtures use hard fescue, but some instead use Kentucky 31 fescue. Kentucky 31 fescue is considered a tall fescue and is a threat level 1 species (WVDNR Natural Heritage Program 2009). Crownvetch and reed canarygrass are also threat level 1 species and should not be used in seeding mixtures (WVDNR Natural Heritage Program 2009). Some of the mixtures use perennial ryegrass and Kentucky bluegrass, which are considered threat level 2 species and are avoided in the developed mixtures (WVDEP 2006 and WVDNR Natural Heritage Program 2009).

\subsection{Conclusion}

The purpose of this chapter was to develop seed mixtures specific to highway reclamation in West Virginia for the WVDOH. In response to the WVDOH request, a total of six mixtures were developed. Plant species in these mixtures were chosen based on their occurrence in the state, species characteristics, seed availability, and purchasing economics. The following main points were determined and developed:

- Species Selection

- For various mixture types

- $\quad$ Seeding Rates

- Seed Mixtures:

- Developed Type A: Mowable Areas

- Developed Type B: Warm Season

- Developed Type C: Cool Season 
○ Developed Type D1: High Elevation $(>2,400 \mathrm{ft}, 731.52 \mathrm{~m})(\leq 3: 1 \mathrm{H}: \mathrm{V})$

○ Developed Type D2: High Elevation $(>2,400 \mathrm{ft}, 731.52 \mathrm{~m})(>3: 1 \mathrm{H}: \mathrm{V})$

- Developed Type E: Wet Areas 


\subsection{Field Testing}

\subsection{Introduction}

Field testing of developed seed mixtures Type A-D under a variety of scenarios was performed at four field sites along Corridor H (U.S. 48) in the Eastern panhandle of West Virginia. The first field site $(\mathrm{CH}-1 \mathrm{~A})$ compared the developed mixtures to the present mixtures used by the WVDOH. At $\mathrm{CH}-2$, the field testing evaluated the need for a high elevation seed mixture. At field site $\mathrm{CH}-3$, the testing determined if seed bed preparation influenced proposed seed germination and cover. The fourth objective evaluated the performance of soil amendments in comparison to topsoil and no topsoil on seed germination and cover at field site $\mathrm{CH}-1 \mathrm{~B}$. Each field site was composed of multiple treatment subplots and monitored on a biweekly approximate schedule. All data collected over the 90 day examination period was examined and analyzed for treatment comparison.

\subsection{Objectives}

The research tested the performance of the experimental seed mixtures in the field. Specific objectives included:

1. Compare the proposed seed mixtures to the current standard.

2. Evaluate the need for a high elevation seed mixture.

3. Determine if seed bed preparation influences proposed seed germination and cover.

4. Evaluate the performance of soil amendments in comparison to topsoil and no topsoil. In the following sections, the field methods are divided into study design and construction and data collection. The study design and construction is described for each objective (1-4). The results section is also broken into and discussed based on objective (1-4). Each objective is then discussed based on vegetation measurements and monitoring data results. Chapter 4 is then concluded with a brief discussion and conclusion. 


\subsection{Methods}

\subsubsection{Study Design and Construction}

\subsubsection{Objective 1: Comparison with current standard}

\section{Site Selection and Layout}

The first step of objective 1 was to choose the site location with the assistance of the WVDOH on March 23, 2015. The site selected was N3907'55.6”, W7858'55.3" (Figure 6), directly beside county road $220 / 8$ (Fish Pond Road). The site had a slope of $5.9 \%$, elevation of $846 \mathrm{ft}(258 \mathrm{~m})$, and slope exposure of $35^{\circ}$ NE. The study site can be seen in Figure 7. Objective 1 examined the performance of WVDOH Type B and Type D and Developed Types A-D. The study site was named $\mathrm{CH}-1 \mathrm{~A}$ and the subplots were $5.4 \mathrm{ft} \times 6 \mathrm{ft}(1.66 \mathrm{~m} \times 1.83 \mathrm{~m})$. Each subplot was replicated three times, totalling 18 subplots. Plot layout can be seen in Figure 8 . The subplot treatments were randomzied as shown in Figure 8. 


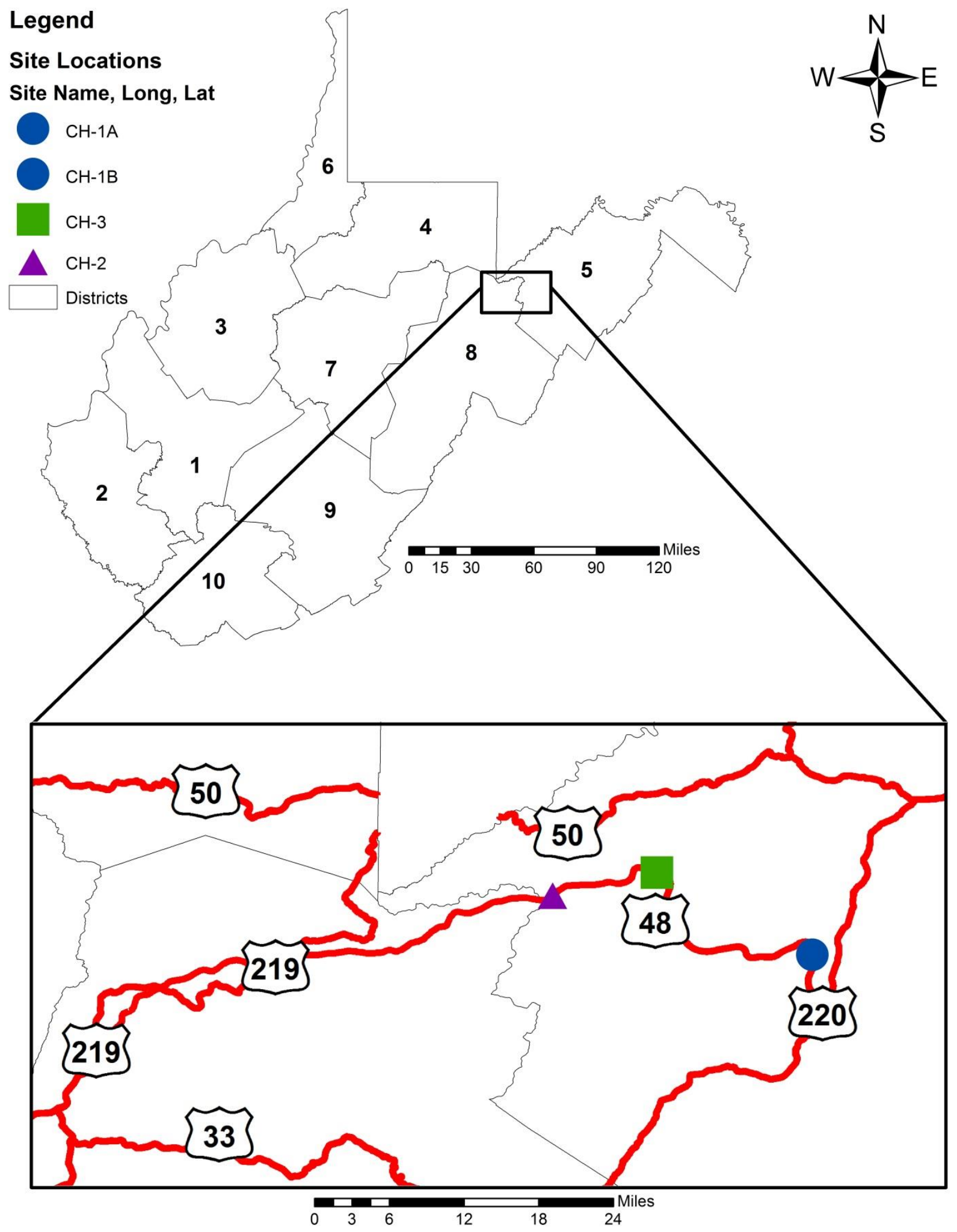

Figure 6: Site Locations 


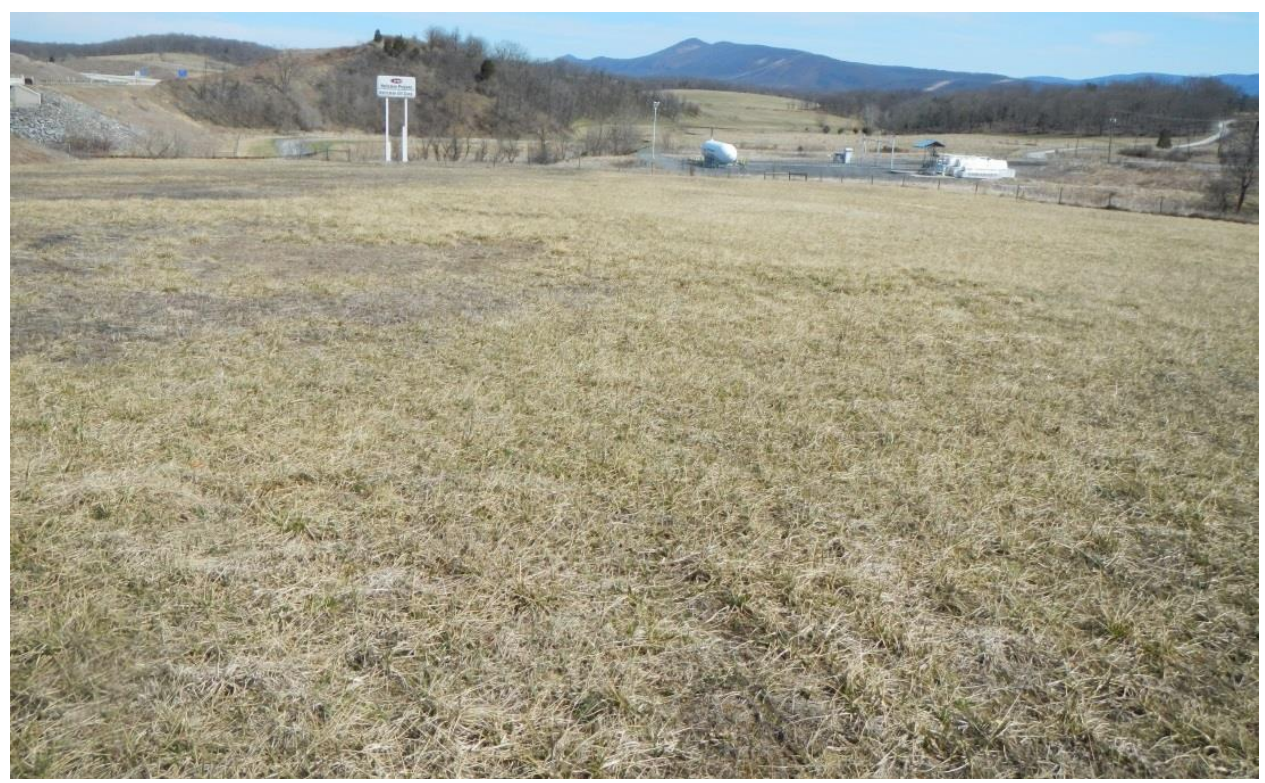

Figure 7: $\mathrm{CH}-1 \mathrm{~A}$ Study Site

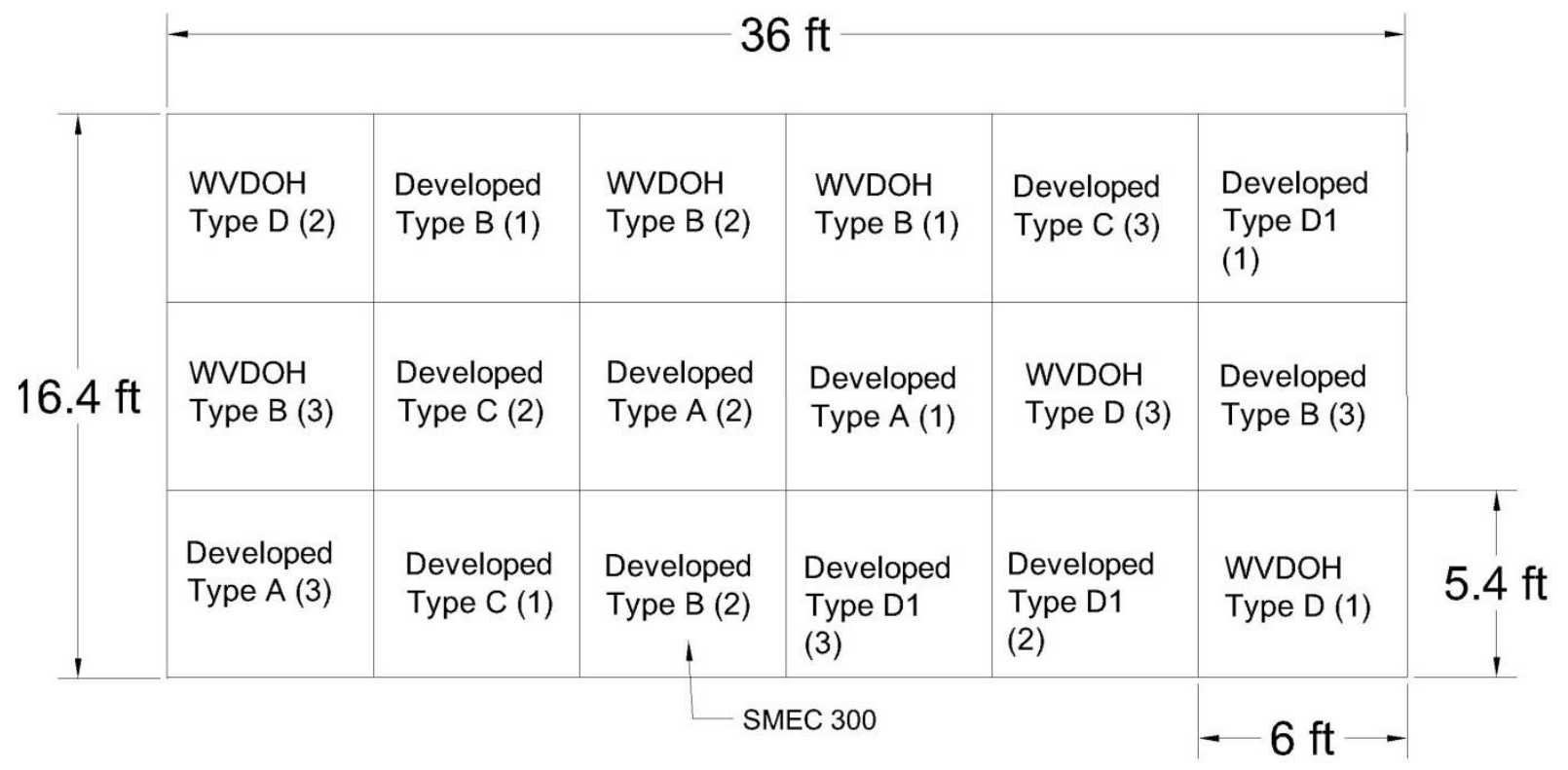

Figure 8: $\mathrm{CH}-1 \mathrm{~A}$ Subplot Layout

\section{Site Preparation}

On April 10, 2015, black plastic (Figure 9) was installed to kill the existing vegetation to prepare the site for treatment application in May. Herbicides were not used in order to avoid any potential affects to the treatments. Fence installation process began in May and was composed of $10 \mathrm{ft}$ tall 4 in. $x 4$ in. ( $3 \mathrm{~m}$ tall $0.1 \mathrm{~m} \times 0.1 \mathrm{~m}$ ) wood treated posts, $8 \mathrm{ft}(2.4 \mathrm{~m}) \mathrm{T}$-posts, and 3.9 $\mathrm{ft}(1.2 \mathrm{~m})$ woven field fence. The final fence height was $7.8 \mathrm{ft}(2.4 \mathrm{~m})$ to keep wildlife out of the 
study area and a visual indicator to the WVDOH mowers to avoid the research area. The fence can be seen in Figure 10 with red flagging tape to aid in deer deterrent and visibility.

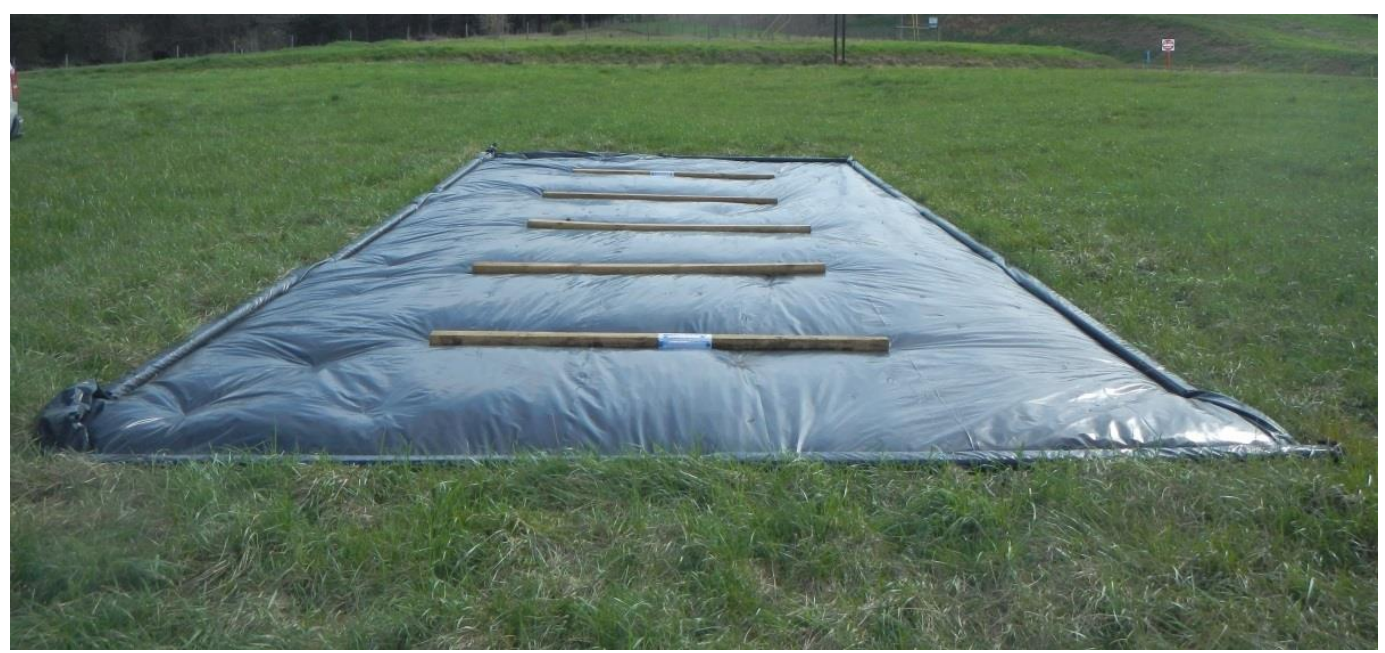

Figure 9: $\mathrm{CH}-1 \mathrm{~A}$ Vegetation Removal

Final vegetation removal was performed on May 15, 2015 by raking the dead vegetation off of the study area with steel garden rakes. Once the existing vegetation material was removed, the black plastic was re-installed until seedbed preparation and planting occurred.

On May 19, 2015, seedbed preparation began by removing the black plastic. The next step was to prepare the soil by light tillage using a Honda FRC800 tiller. During the tilling process, all rock and debris greater than 2 in. $(0.05 \mathrm{~m})$ were removed by hand in accordance to the WVDOH 652 specifications. Then, a $300 \mathrm{lb}$ (136 kg) Sakai PF150 plate compactor was used to re-compact the soil to mimic earthwork construction. Finally, the soil surface was scarified using a steel garden rake to a depth of 0.5-1 in. (0.01-0.03 m). The prepaired seedbed can be seen in Figure 10. 


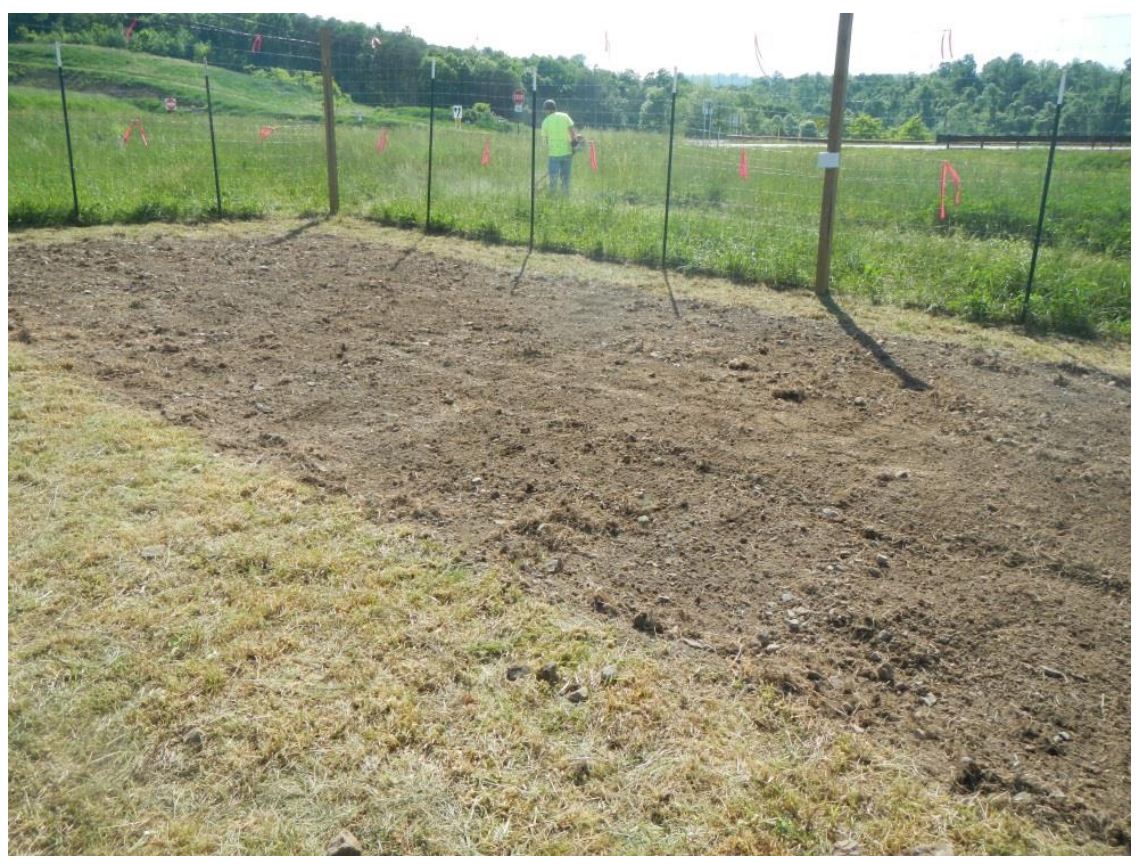

Figure 10: $\mathrm{CH}-1 \mathrm{~A}$ Seedbed Preparation

\subsection{Equipment Installation}

After seedbed preparation, a grid system and research equipment were installed. The grid system was composed of twenty-two 8 in. $(0.2 \mathrm{~m})$ landscape stakes and twisted mason line. The grid system was laid out like the suplot design in Figure 8 . The stakes were held $1 \mathrm{ft}$ $(0.3 \mathrm{~m})$ from the edged of the tilled plot area to ensure minimal distrubance of the subplots. Once the stakes were installed, twisted mason line was run between the stakes to create the grid system seen in Figure 11 and 12.

The research equipment installed entailed a WatchDog Tipping Bucket Rain Gauge, WaterScout SMEC 300, and WatchDog 1425 Micro Station. Equipment details and use are specified in Section 4.3.2. The WatchDog Tipping Bucket Rain Gauge mounting bracket was leveled and installed on the top of one of the treated wood posts. The bracket was kept at minimum $1 \mathrm{ft}(0.3 \mathrm{~m})$ from the metal fence by recommendation of the manufacturer. After the bracket was mounted, the rain gauge was installed and the cable ran down the post to the WatchDog 1425 Micro Station enclosure. The enclosure was mounted $4 \mathrm{ft}(1.2 \mathrm{~m})$ up the post 
for easy access and data collection for future visits. The WatchDog 1425 Micro Station was put into the enclosure for safe storage and protection from enviromental elements.

The WaterScout SMEC 300 was then installed into subplot "Developed Type B (2)" seen in Figure 8. The installation was conducted by placing the sensor $0.5 \mathrm{ft}(0.15 \mathrm{~m})$ into the soil media. The sensor was placed in the soil perpendicular to the surface and firmly pressed into the soil face after a slot was made with a knife. The slot for the sensor was necessary to avoid braking the SMEC 300, seen in Figure 39 and 40. CARLON Ent. Conduit was used to run the SMEC 300 and rain gauge wire through to protect them from potential weathering and vermin damage. The conduit was buried $0.5 \mathrm{ft}(0.15 \mathrm{~m})$ in the soil and ran up and down the post to the Micro Station enclosure from the rain gauge and SMEC 300.

The SMEC 300 was plugged into channel $D$ and the rain gauge plugged into channel $A$. The WatchDog 1425 Micro Station was then plugged into a laptop and launched with SpecWare 9 Professional. All the equipment was initially setup in the laboratory to verify calibration was correct. The field setup simply launched the equipment to begin data recording and verified everything was working correctly in the field.

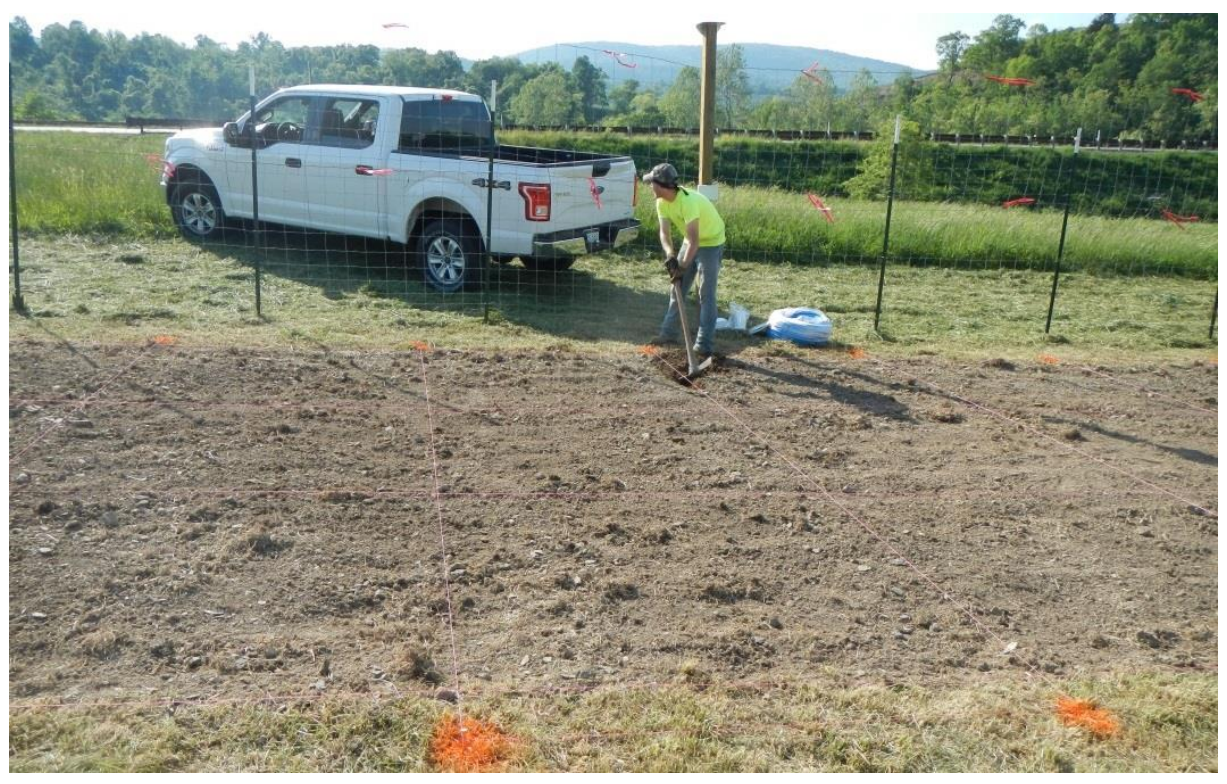

Figure 11: $\mathrm{CH}-1 \mathrm{~A}$ Equipment Installation 


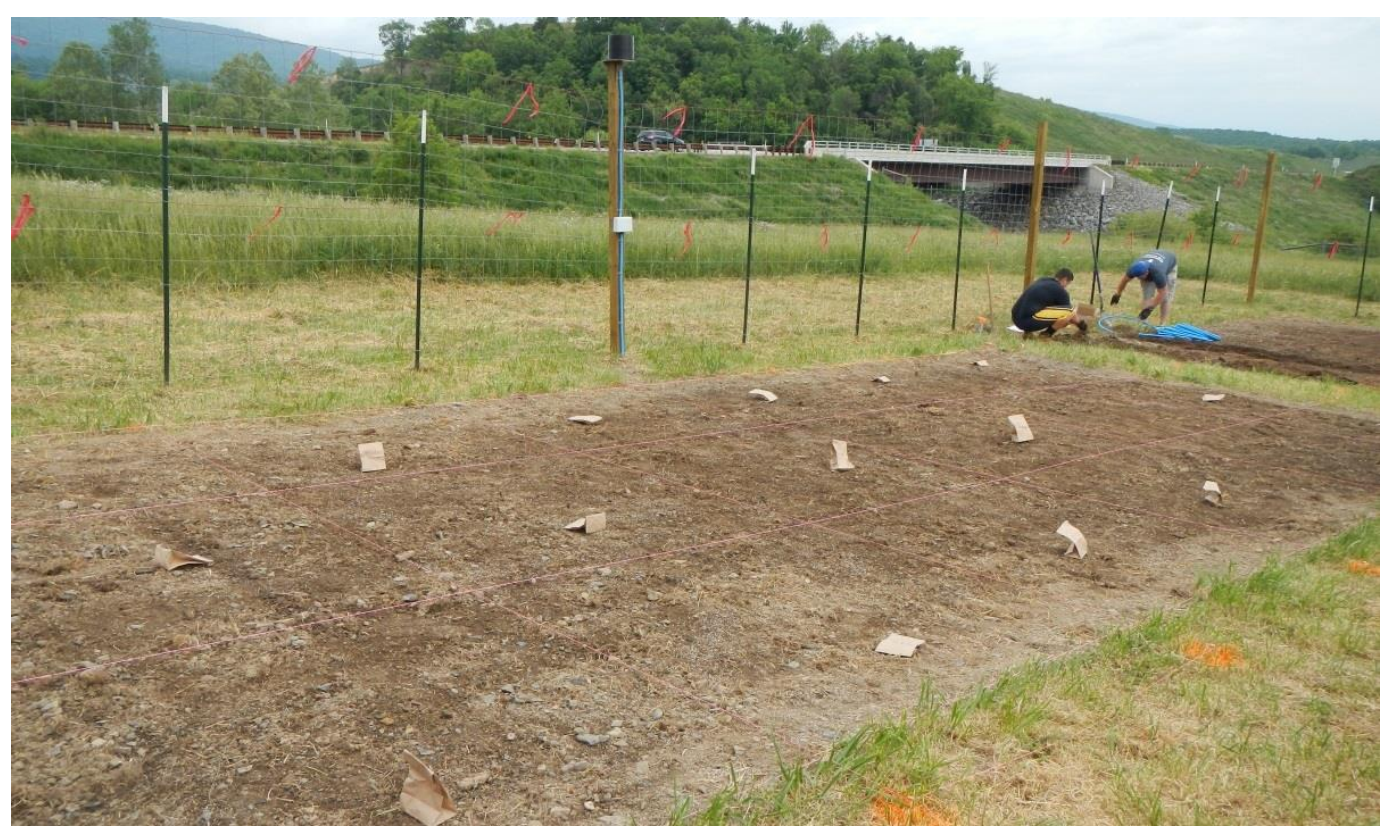

Figure 12: $\mathrm{CH}-1 \mathrm{~A}$ Grid Installation

\section{Fertilizer and Seed Application}

Fertilizer application recommendation for $\mathrm{CH}-1 \mathrm{~A}$ was determined from the soil test seen

in Table 18. The fertilizer used was a $10-10-10$ with a total of $6.61 \mathrm{lb}(2.99 \mathrm{~kg})$ for the entire $\mathrm{CH}-$ 1A site displayed in Table 18. The appropriate quantity was measured with an Ohaus Explorer Model E14130 in the laboratory and stored in a paper bag until application. The scale had an accuracy of $2.2 \times 10^{-7} \mathrm{lb}(0.1 \mathrm{mg})$. The fertilizer was applied by a homemade shaker displayed in Figure 13. The shaker allowed for an approximate even distribution due to the small subplot sizes.

Table 18: Fertilizer Application Quantity

\begin{tabular}{cccccccc}
\hline $\begin{array}{l}\text { Site } \\
\text { Location }\end{array}$ & $\begin{array}{c}\text { Experimental } \\
\text { Area }\end{array}$ & $\begin{array}{c}\text { Estimated } \\
\text { Area }\end{array}$ & \multicolumn{2}{c}{ Soil Test Results } & $\begin{array}{c}\text { Ratio } \\
\text { Available }\end{array}$ & $\begin{array}{c}\text { Quantity } \\
\text { Needed }\end{array}$ \\
\hline & & & $\mathrm{N}$ & $\mathrm{P}_{2} \mathrm{O}_{5}$ & $\mathrm{~K}_{2} \mathrm{O}$ & $\mathrm{N}$ \\
Units & $\mathrm{ft}^{2}$ & acre & & $\mathrm{lb} / \mathrm{acre}$ & & $\mathrm{lb}$ \\
$\mathrm{CH}-1 \mathrm{~A}$ & 576 & 0.0132 & 50 & 50 & 50 & $10-10-10$ & 6.61 \\
\hline
\end{tabular}


Seed application began by determining the correct application amount for each subplot. Seed mixture application quantities for seed and inoculant with an application area of $98.67 \mathrm{ft}^{2}$ $\left(9.17 \mathrm{~m}^{2}\right)$ :

- WVDOH Type B

○ Application amount $(0.205 \mathrm{lb})(3-31.15$ gram bags) $(+/-0.030 \mathrm{~g})$

○ Inoculant $(0.234 \mathrm{~g})(+/-0.030 \mathrm{~g})$

- WVDOH Type D

○ Application amount (0.142 lbs.) (3- 21.62 gram bags) $(+/-0.030 \mathrm{~g})$

- Inoculant $(0.162 \mathrm{~g})(+/-0.030 \mathrm{~g})$

- Developed Type A

○ Application amount (0.367 lbs.) (3- 55.79 gram bags) $(+/-0.030 \mathrm{~g})$

○ Inoculant $(0.418 \mathrm{~g})(+/-0.030 \mathrm{~g})$

- Developed Type B

○ Application amount (0.161 lbs.) (3- 24.49 gram bags) $(+/-0.030 \mathrm{~g})$

$\circ$ Inoculant $(0.184 \mathrm{~g})(+/-0.030 \mathrm{~g})$

- Developed Type C

○ Application amount (0.124 lbs.) (3- 18.90 gram bags) $(+/-0.030 \mathrm{~g})$

- Inoculant $(0.142 \mathrm{~g})(+/-0.030 \mathrm{~g})$

- Developed Type D1

○ Application amount (0.113 lbs.) (3- 17.24 gram bags) (+/- $0.030 \mathrm{~g})$

$\circ$ Inoculant $(0.129 \mathrm{~g})(+/-0.030 \mathrm{~g})$

With three subplots, the application amount was divided into three equal parts, weighed, mixed with the correct inoculant, and stored in a paper bag until planting. Weighing and mixing of the seed and inoculant took place one week before planting to ensure survivability of the inoculant. The weight accuracy of the user was within $+/-6.6 \times 10^{-5} \mathrm{lb}(+/-0.030 \mathrm{~g})$ to the specified amount above. Ohaus Explorer Model E14130 was used to weigh the seed, and inoculant. The scale had an accuracy of $2.2 \times 10^{-7} \mathrm{lb}(0.1 \mathrm{mg})$.

Seed application occurred on May 27, 2015 by using a fabricated shaker to get accurate distribution for the small subplots (Figure 13). Before application, the seed mixtures, in paper bags, were placed in their corresponding subplot (Figure 12) for organization and easy identification and application. Once the seed mixtures were applied, a steel garden rake was used to scuff the surface to promote seed to soil contact. 


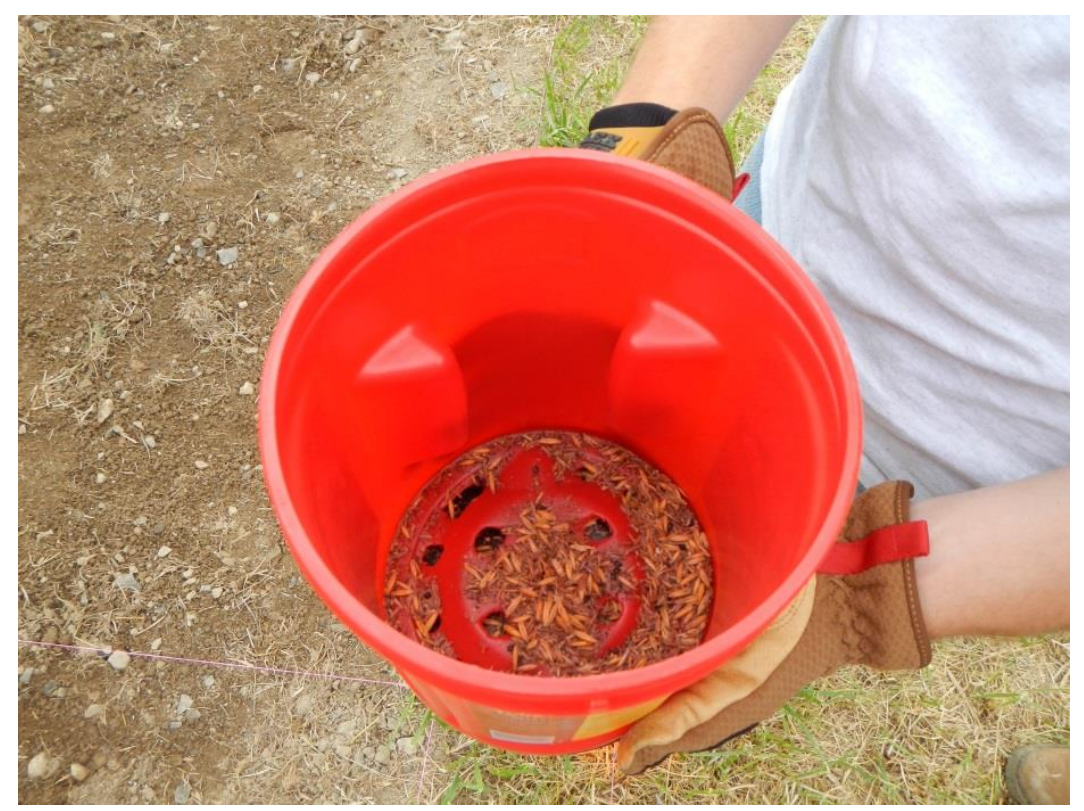

Figure 13: CH-1A Seed Application

After the seed mixtures were planted, the black tarp was re-applied to the plot area until the HECP could be applied the next morning. The plastic was placed back onto the plot due to the threat of birds and heavy rain overnight. The seed was applied the day before HECP application to reduce wait time for Arnold's Custom Seeding, LLC (ACS) who donated their time to come to the area to apply the HECP product donated by Profile Products, LLC.

\section{HECP Application}

On May 28, 2015, Arnold's Custom Seeding, LLC (ACS) arrived on site at 8:00 am to begin HECP application. The HECP used was ProMatrix ${ }^{\mathrm{TM}}$ Engineered Fiber Matrix (EFM) by Profile Products, LLC. at an application rate of $3,000 \mathrm{lb} / \mathrm{ac}(3,400 \mathrm{~kg} / \mathrm{ha})$. Profile Products, LLC attended application of their product to verify proper application rate and technique was performed (Profile Products LLC. 2015a). Application of ProMatrix can be seen in Figure 14 by a FiNN T170 Hydro Seeder owned and operated by ACS. Excess product was mixed in the hydro seeder to ensure proper mixing of the product and application abilities of the machine. 


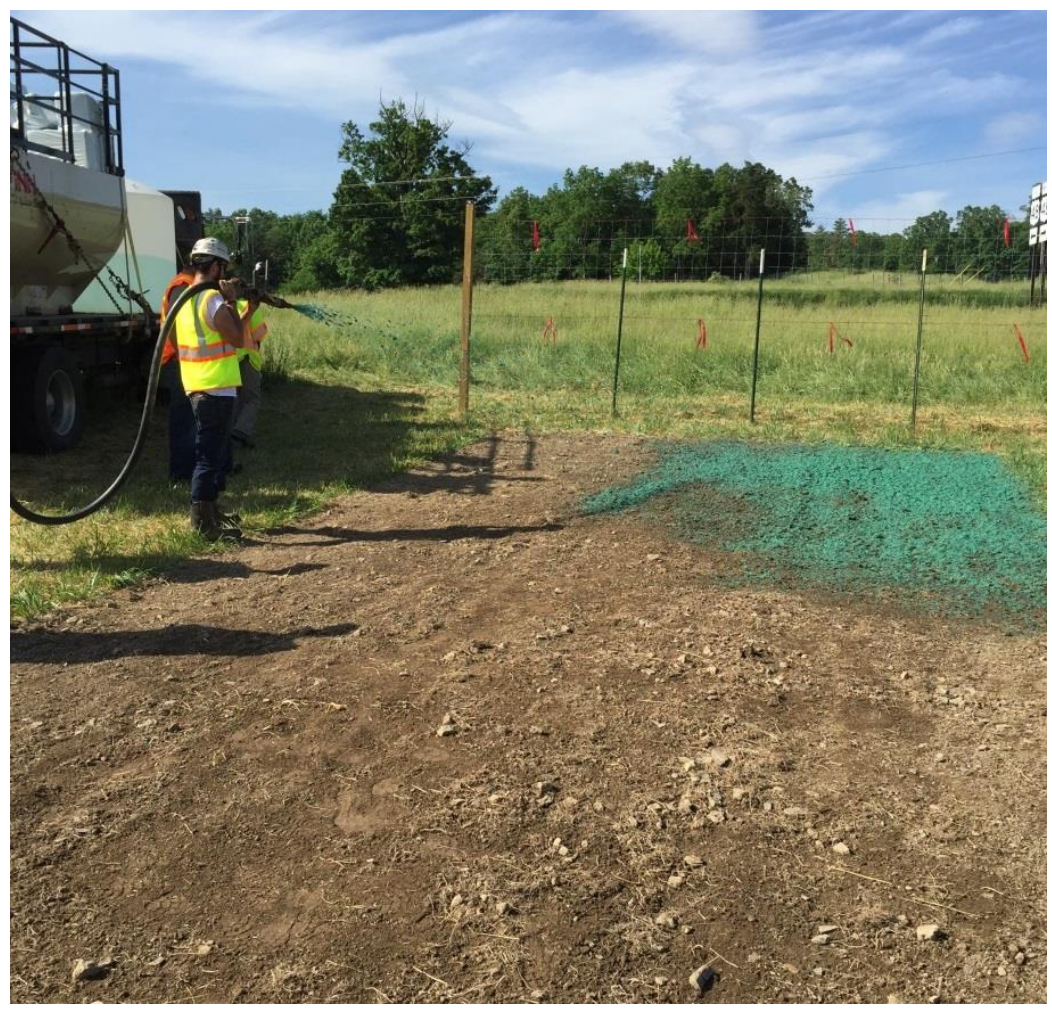

Figure 14: $\mathrm{CH}-1 \mathrm{~A}$ HECP Application

ProMatrix is an EFM that can be used for erosion control practices specifying the use of a stabilized mulch matrix or bonded fiber matrix. The product contains recycled wood fibers and is non-toxic and biodegradable. The recommended application for slopes less than $3 \mathrm{H}: 1 \mathrm{~V}$ is $3,000 \mathrm{lb} / \mathrm{ac}(3,400 \mathrm{~kg} / \mathrm{ha})$ at a mixing ratio of $60 \mathrm{lb}(27 \mathrm{~kg})$ of product to $100 \mathrm{gal}$ (379 liters) of water. The product can be used for slopes less than $1 \mathrm{H}: 1 \mathrm{~V}$, but take note that steeper slopes require higher application rates to justify appropriate erosion control (Profile Products LLC. 2015a). A picture of the sprayed product can be seen in Figure 15 and the completed $\mathrm{CH}-1 \mathrm{~A}$ site can be seen in Figure 16. 


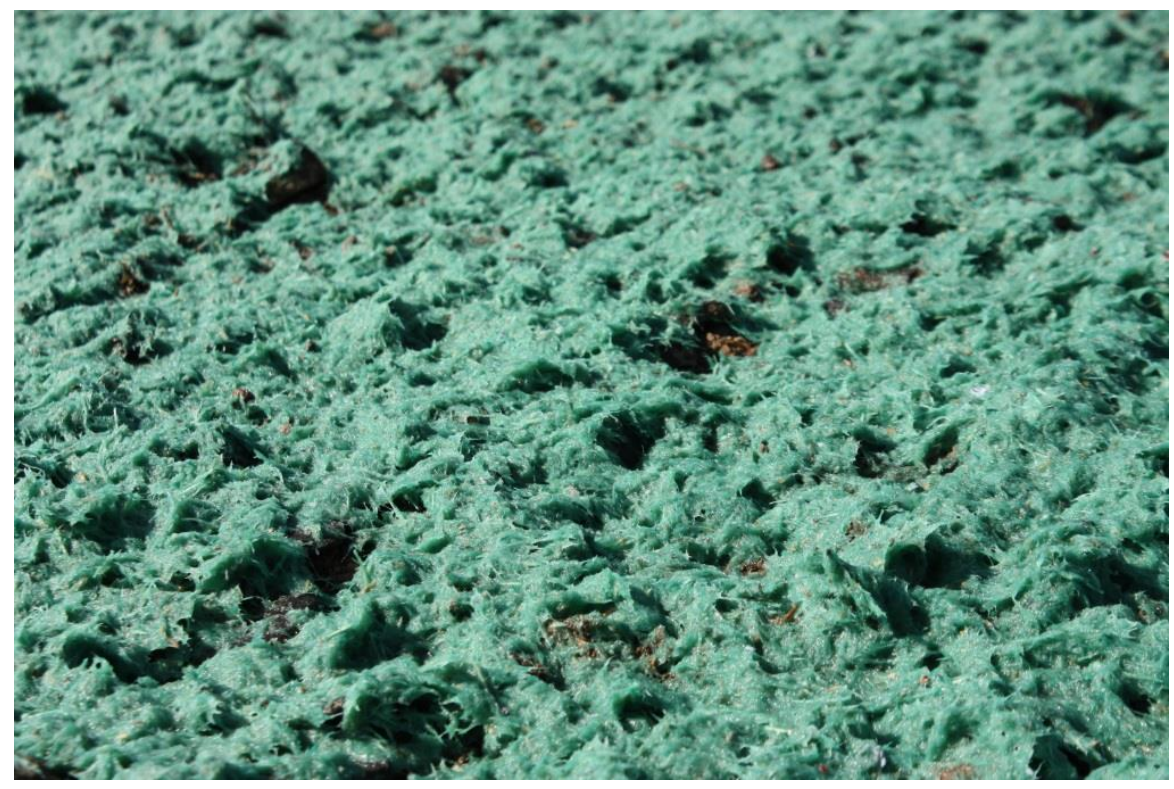

Figure 15: CH-1A HECP (Photo by Profile Products, Adam Dibble/Matt Welch)

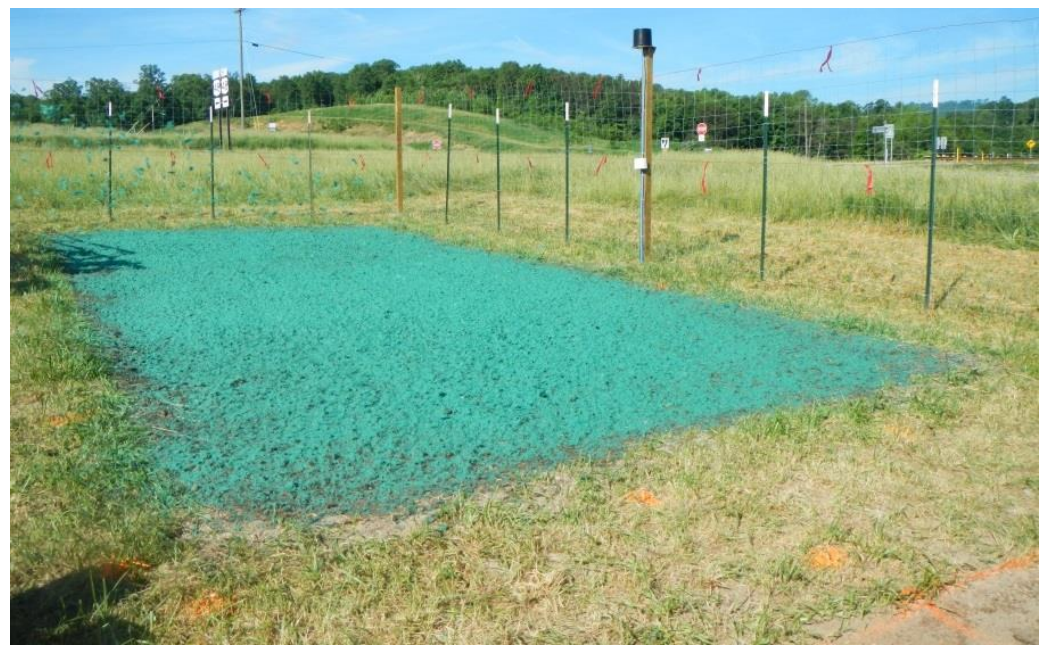

Figure 16: CH-1A Preparation Completion

\section{Weed Removal}

Due to large numbers of Jimson weed (Datura stramonium) and small subplot areas, the species had to be removed to address overcrowding concerns on June 24, 2015. Seen in Figure 17, the plant heavily occupied the subplots and in some cases were $2 \mathrm{ft}$ tall and wide. The plot area before plant removal can be seen in Figure 18. After species removal seen in Figure 19, a significant difference in overall plot area coverage was evident. No other species removal occurred through the remainder of the study. 


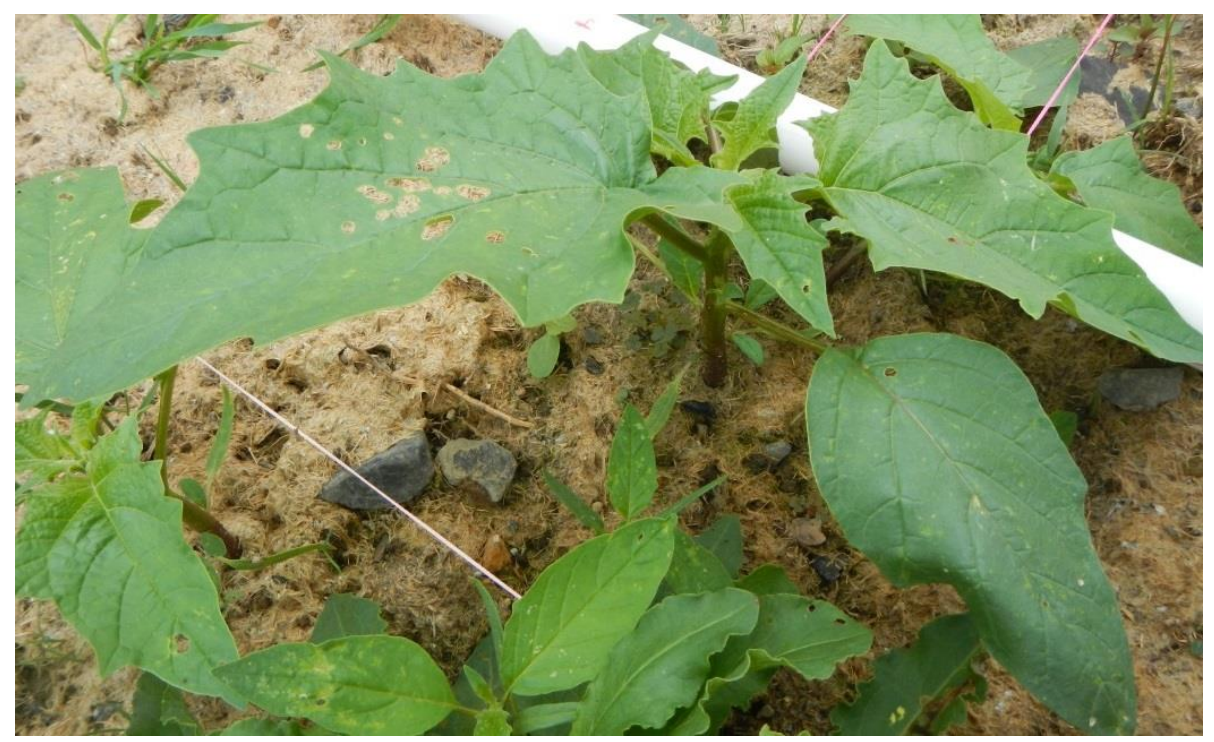

Figure 17: Jimson Weed (Datura stramonium)

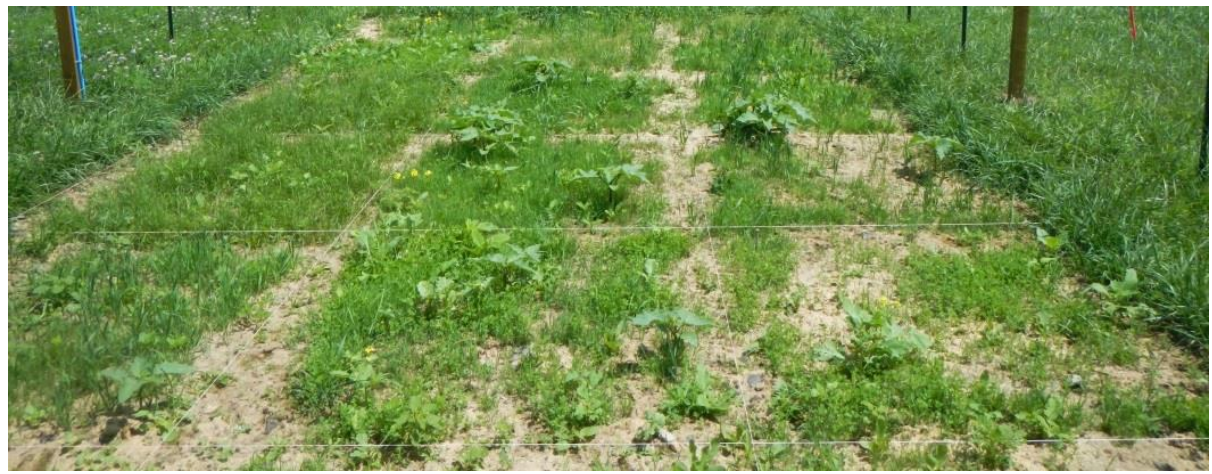

Figure 18: $\mathrm{CH}-1 \mathrm{~A}$ Before Weed Removal

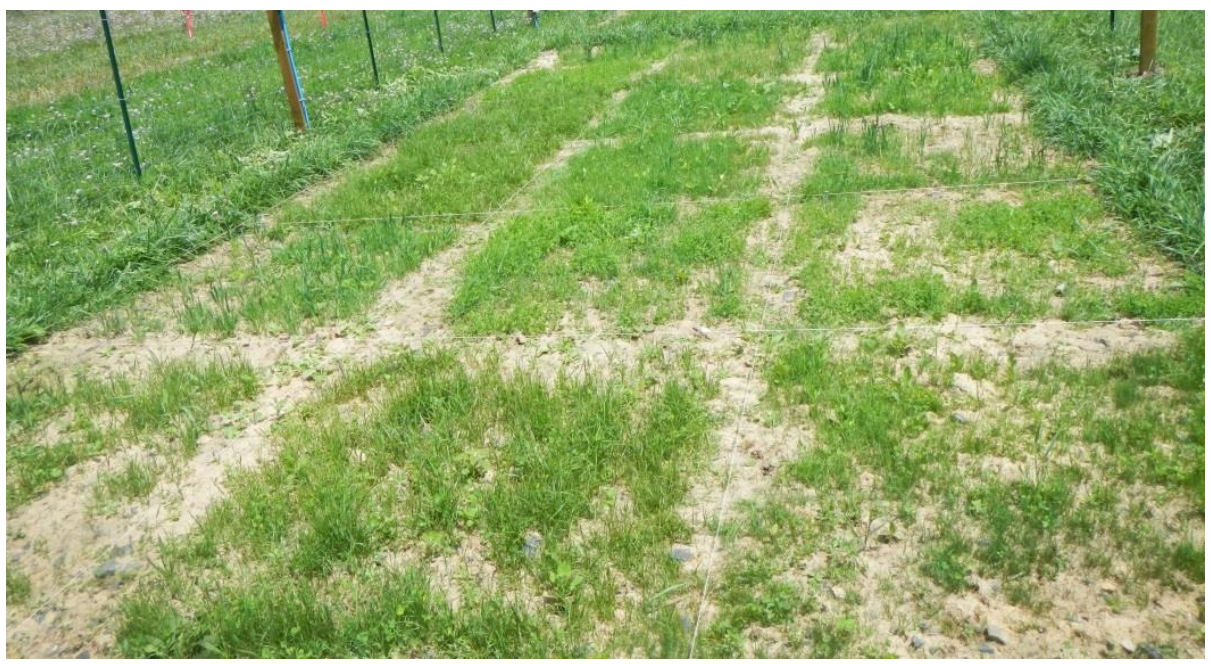

Figure 19: CH-1A After Weed Removal 


\subsubsection{Objective 2: Evaluating high elevation needs}

\section{Site Selection and Layout}

Objective 2 examined the performance of WVDOH Type B and Developed Types A-D at a high elevation. The site was located at N39 $12^{\prime} 05.4^{\prime \prime}$, W79 $17^{\prime} 0.4^{\prime \prime}$ close to the Mt. Storm power plant off of U.S. 48. The site had a slope of 3.9\%, elevation of $3294 \mathrm{ft}(1004 \mathrm{~m})$, and slope exposure of $326^{\circ} \mathrm{NW}$. The site location can be seen in Figure 6. The study site was named CH-2 (Figure 20). The subplots are $5.4 \mathrm{ft} \times 5.9 \mathrm{ft}(1.66 \mathrm{~m} \times 1.8 \mathrm{~m})$. Each subplot was replicated three times, totalling 15 subplots. Plot layout can be seen in Figure 21. Randomization of sbuplot treatments was conducted the same as in Objective 1 and can be seen in Figure 21.

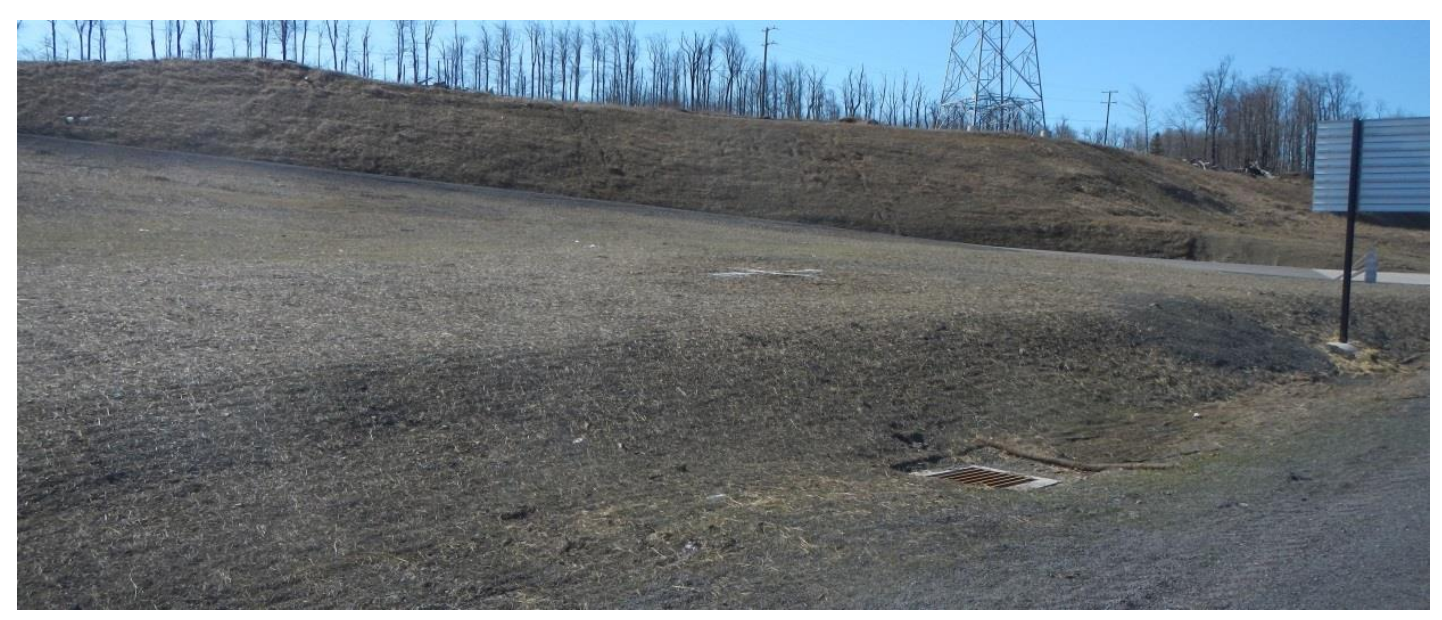

Figure 20: $\mathrm{CH}-2$ Study Site 


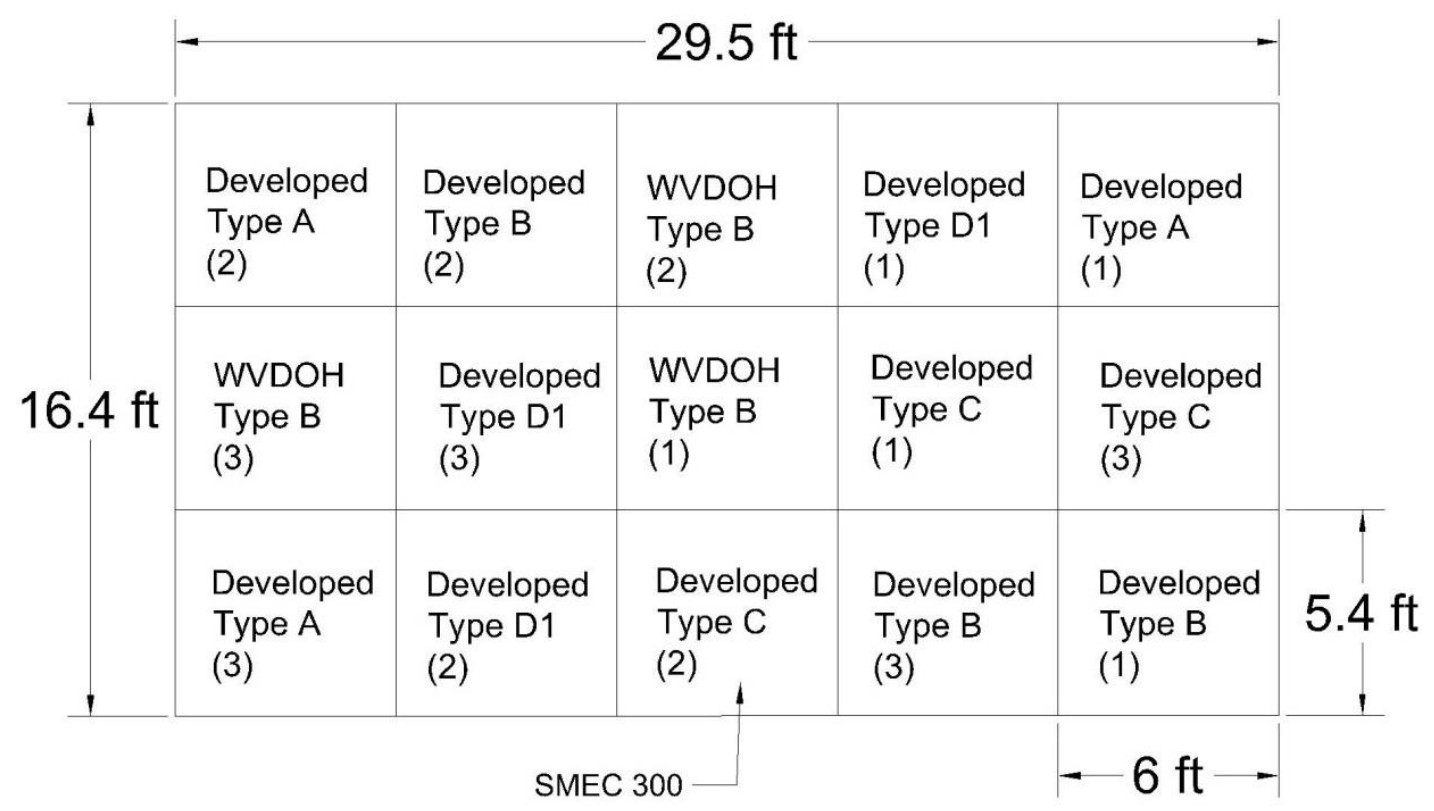

Figure 21: CH-2 Subplot Layout

\section{Site Preparation}

Using methods stated in Section 4.3.1.1, the vegetation was covered (Figure 22) and fence was installed. The fence was composed of $3 \mathrm{~m}$ tall $10 \mathrm{ft}$ tall $4 \mathrm{in}$. $\mathrm{x} 4 \mathrm{in} .(0.1 \mathrm{~m} \times 0.1 \mathrm{~m})$ wood treated posts, $8 \mathrm{ft}(2.4 \mathrm{~m})$ T-posts, and $3.9 \mathrm{ft}(1.2 \mathrm{~m})$ woven field fence. The final fence height was $7.8 \mathrm{ft}(2.4 \mathrm{~m})$ to keep wildlife out of the study area and a visual indicator to the WVDOH mowers to avoid the research area. The fence can be seen in Figure 23 with red flagging tape to aid in deer deterrent and visibility.

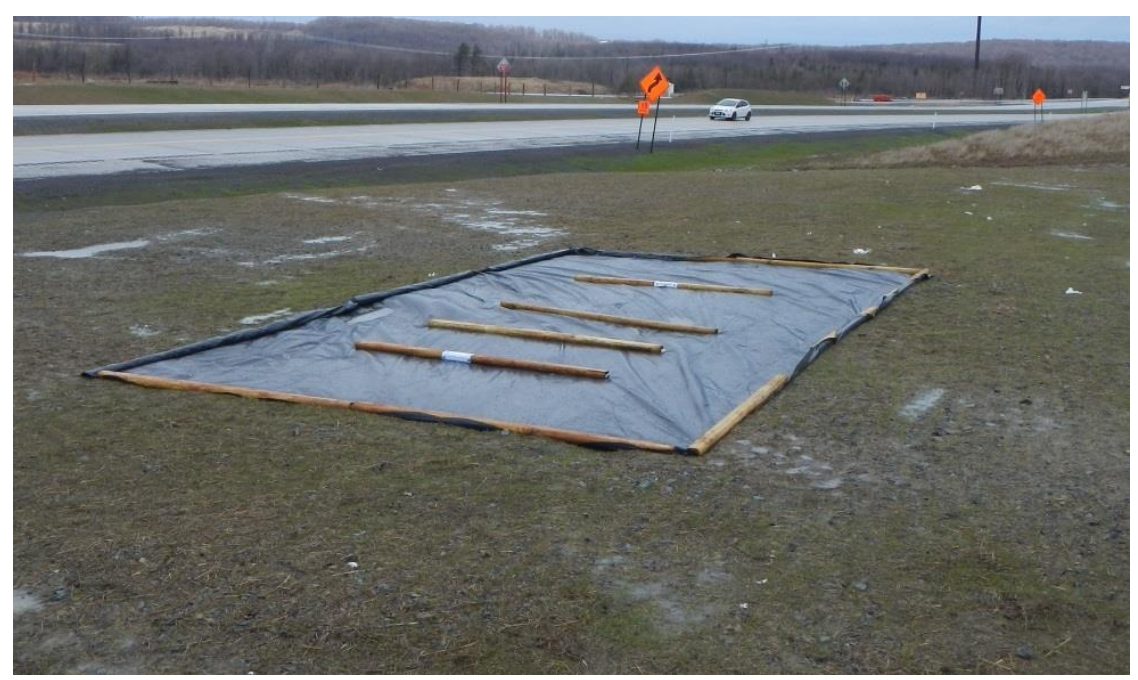

Figure 22: CH-2 Vegetation Removal 


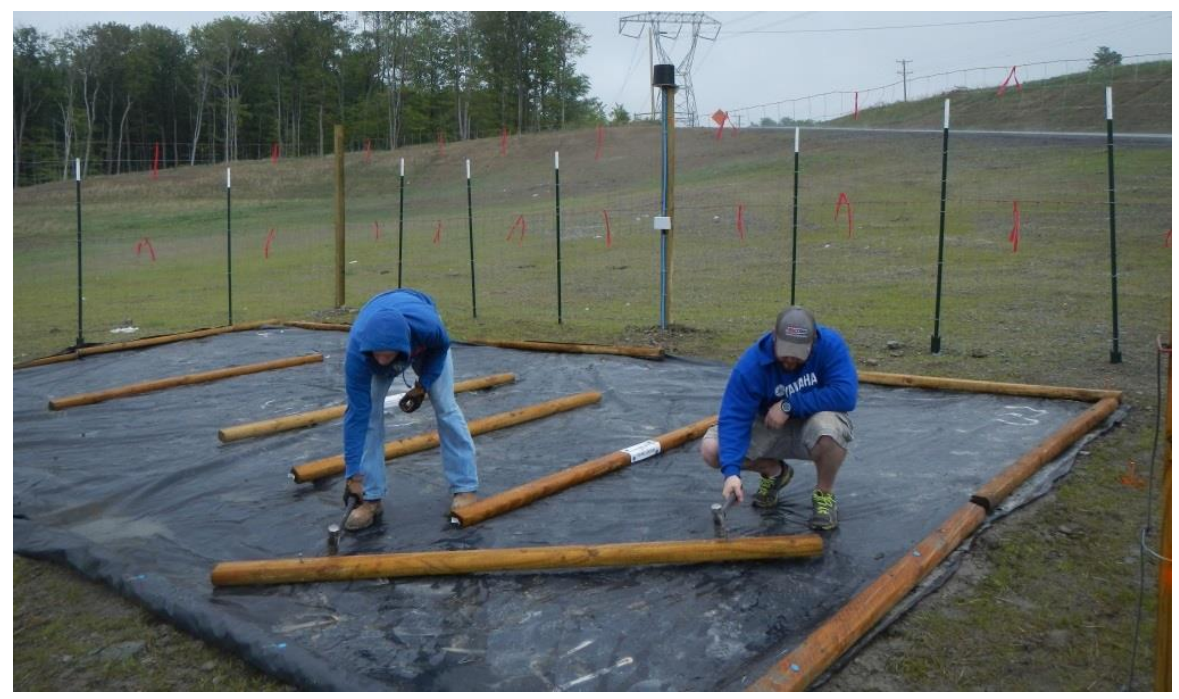

Figure 23: CH-2 Fence Installation and Re-application of Plastic

Final vegetation removal was performed on May 15, 2015 by raking the dead vegetation off of the study area with steel garden rakes. Once the existing vegetation material was removed, the black plastic was re-installed (Figure 24) until seedbed preparation and planting. Seedbed preparation was conducted the same as in Section 4.3.1.1 on May 21, 2015. The prepared seedbed can be seen in Figure 24 .

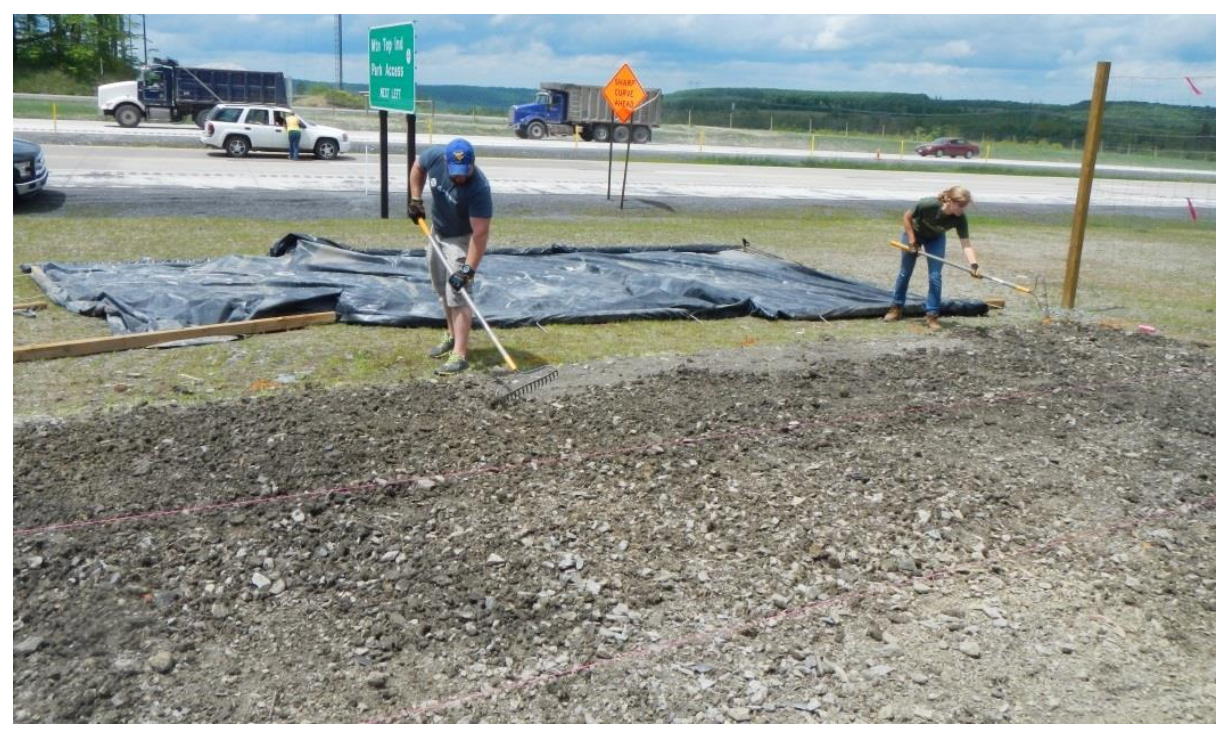

Figure 24: $\mathrm{CH}-2$ Seedbed Preparation

\section{Equipment Installation}

After seedbed preparation, a grid system and research equipment were installed. The research equipment installed was the same entailed in Section 4.3.1.1. The WaterScout SMEC 
300 was installed into subplot "Developed Type C (2)" seen in Figure 21. The grid system was composed of $208 \mathrm{in.}(0.2 \mathrm{~m})$ landscape stakes and twisted mason line seen in Figure 25. The grid system was laid out like the suplot design in Figure 21 and constructed the same as in Section 4.3.1.1.

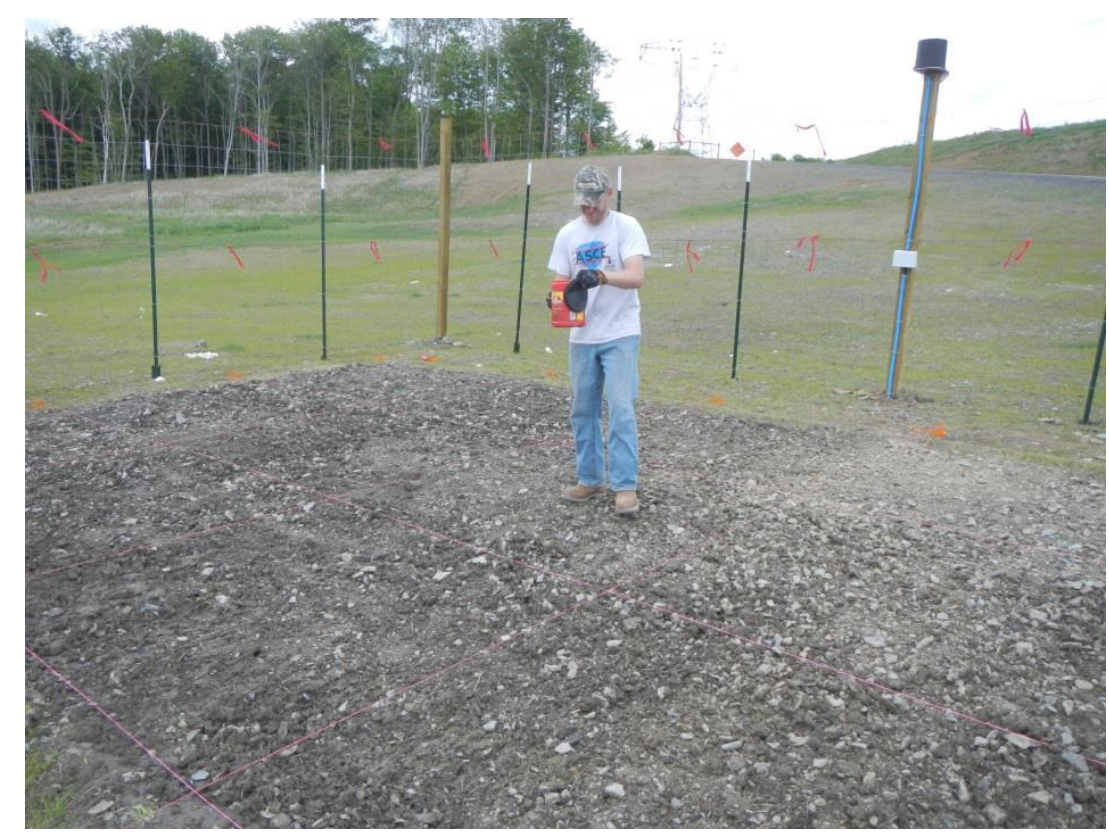

Figure 25: CH-2 Grid System and Seeding

\section{Fertilizer and Seed Application}

Fertilizer and seed was applied to each subplot. Fertilizer application recommendation for $\mathrm{CH}-2$ was determined from the soil test seen in Table 19. Fertilizer type and application recommendation was 35-50-55 lb/ac. The fertilizer used was a 5-10-10 with a total of $5.51 \mathrm{lb}$ $(2.49 \mathrm{~kg})$ for the entire $\mathrm{CH}-2$ site displayed in Table 19. Measurement and application was conducted the same as in Section 4.3.1.1.

Table 19: Fertilizer Application Quantity

\begin{tabular}{cccccccc}
\hline $\begin{array}{l}\text { Site } \\
\text { Location }\end{array}$ & $\begin{array}{c}\text { Experimental } \\
\text { Area }\end{array}$ & $\begin{array}{c}\text { Estimated } \\
\text { Area }\end{array}$ & \multicolumn{2}{c}{ Soil Test Results } & $\begin{array}{c}\text { Ratio } \\
\text { Available }\end{array}$ & $\begin{array}{c}\text { Quantity } \\
\text { Needed }\end{array}$ \\
\hline & & & $\mathrm{N}$ & $\mathrm{P}_{2} \mathrm{O}_{5}$ & $\mathrm{~K}_{2} \mathrm{O}$ & & $\mathrm{N}$ \\
Units & $\mathrm{ft}^{2}$ & acre & & $\mathrm{lb} / \mathrm{acre}$ & & $\mathrm{lb}$ \\
$\mathrm{CH}-2$ & 480 & 0.0110 & 35 & 50 & 55 & $5-10-10$ & 5.51 \\
\hline
\end{tabular}


Seed application began by determining the correct application amount for each subplot.

Seed mixture application quantities for seed and inoculant:

- WVDOH Type B

○ Application amount (0.202 lbs.) (3- 30.54 gram bags) (+/- $0.030 \mathrm{~g})$

○ Inoculant $(0.229 \mathrm{~g})(+/-0.030 \mathrm{~g})$

- Developed Type A

○ Application amount (0.361 lbs.) (3- 54.58 gram bags) $(+/-0.030 \mathrm{~g})$

- Inoculant $(0.409 \mathrm{~g})(+/-0.030 \mathrm{~g})$

- Developed Type B

○ Application amount (0.159 lbs.) (3- 24.04 gram bags) $(+/-0.030 \mathrm{~g})$

- Inoculant $(0.180 \mathrm{~g})(+/-0.030 \mathrm{~g})$

- Developed Type C

$\circ$ Application amount (0.122 lbs.)(3- 18.45 gram bags) $(+/-0.030 \mathrm{~g})$

- Inoculant $(0.138 \mathrm{~g})(+/-0.030 \mathrm{~g})$

- Developed Type D1

○ Application amount (0.111 lbs.) (3- 16.78 gram bags) (+/- $0.030 \mathrm{~g})$

$\circ$ Inoculant $(0.126 \mathrm{~g})(+/-0.030 \mathrm{~g})$

Seed measurement method, application, and re-application of the black tarp was performed the same as in Section 4.3.1.1. Figure 26 shows the seed mixture application and preparation layout. Once the seed mixtures were applied, a steel garden rake was used to scuff the surface to promote seed to soil contact.

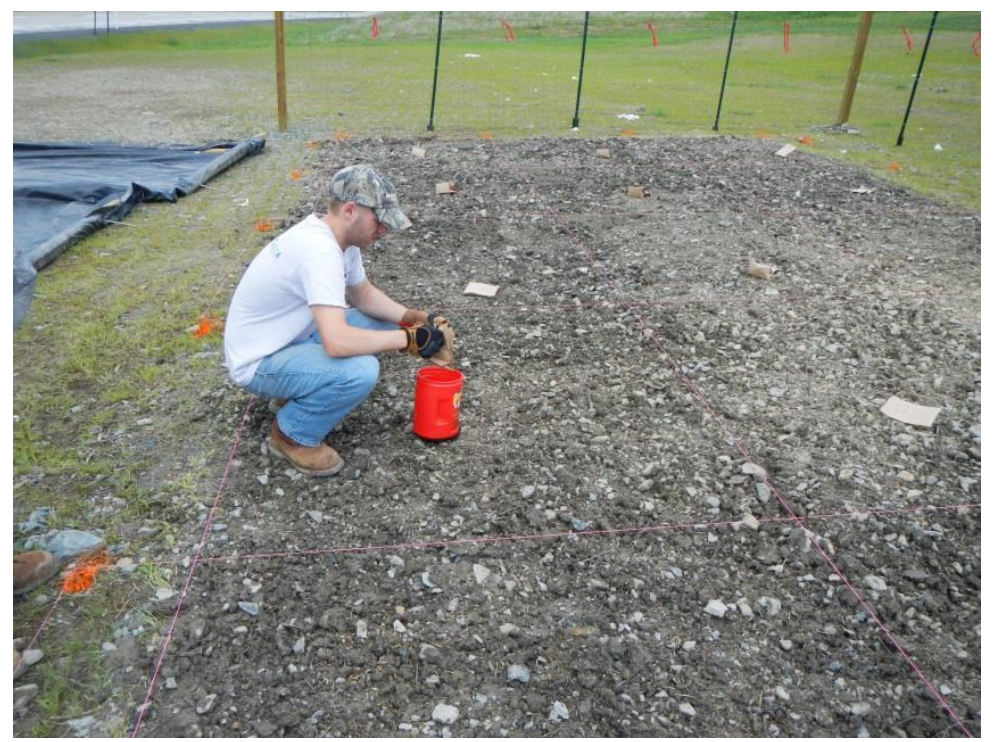

Figure 26: CH-2 Seed Bags 


\section{HECP Application}

HECP was applied in accordance to Section 4.3.1.1 with identical product descriptions.

Application of ProMatrix can be seen in Figure 27. An up close picture of the sprayed product can be seen in Figure 28 and the completed CH-2 site can be seen in Figure 29.

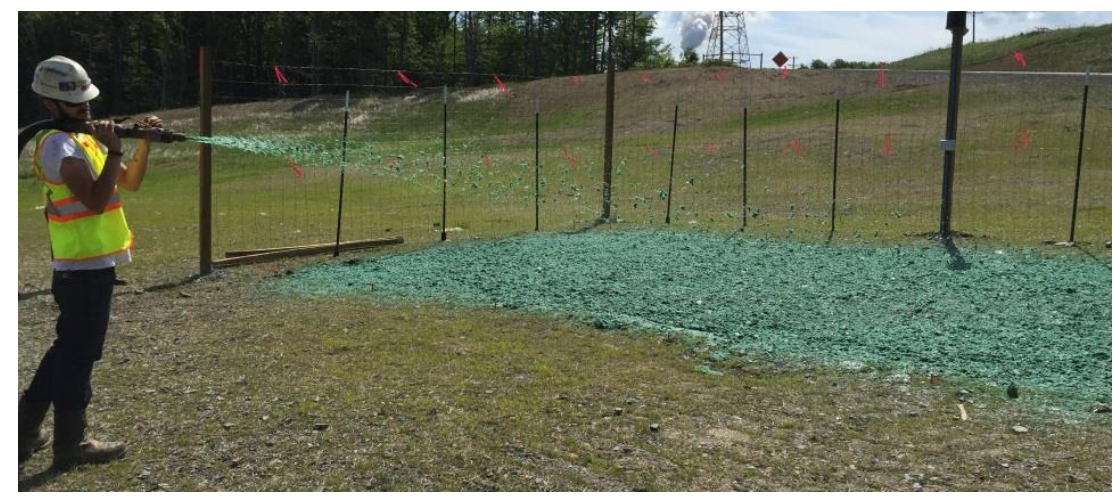

Figure 27: CH-2 HECP Application

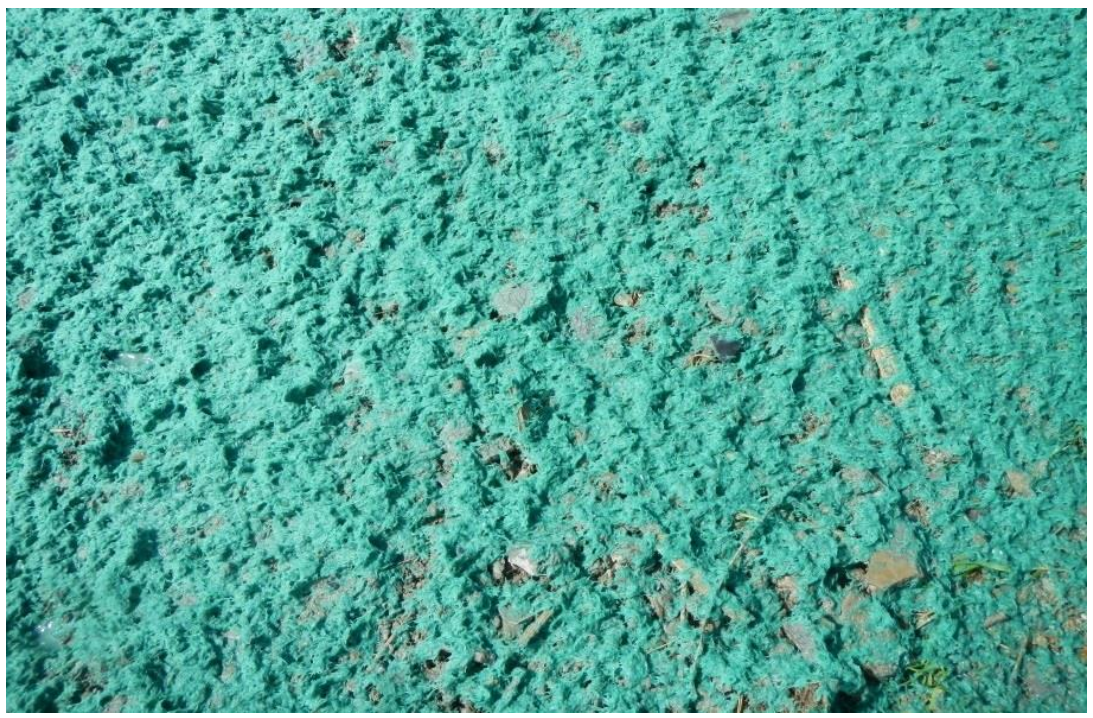

Figure 28: $\mathrm{CH}-2$ HECP 


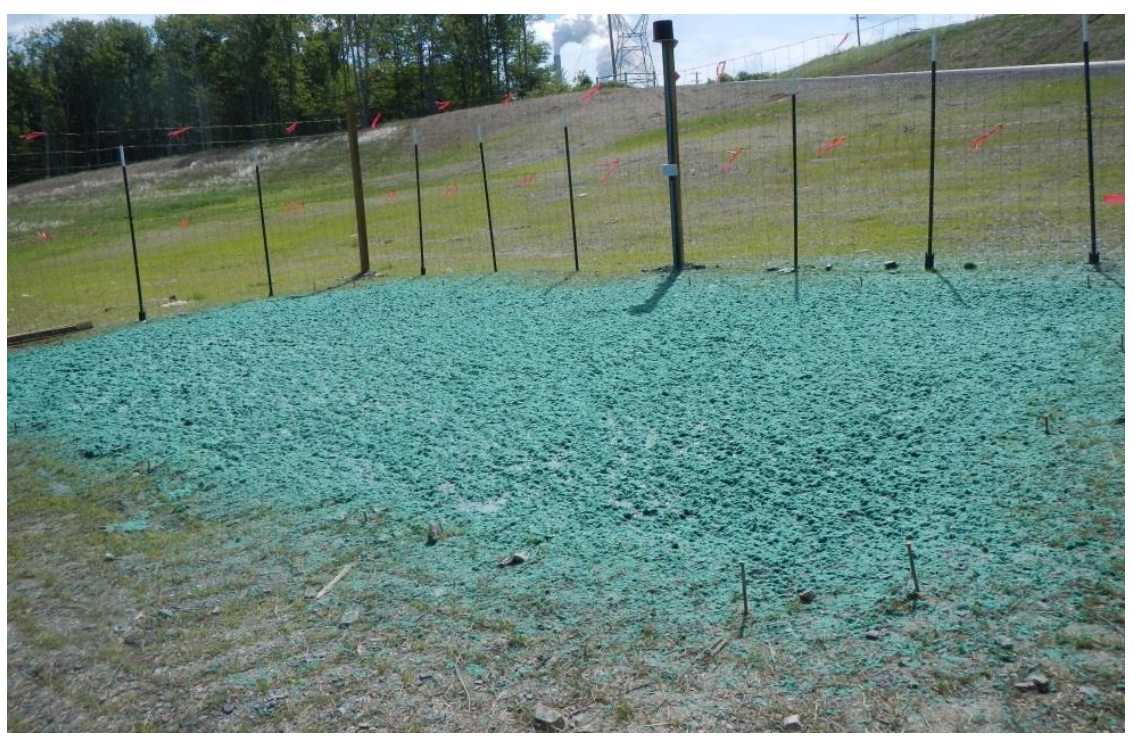

Figure 29: $\mathrm{CH}-2$ Completed Site

\subsubsection{Objective 3: Examining seedbed preparation techniques}

\section{Site Selection and Layout}

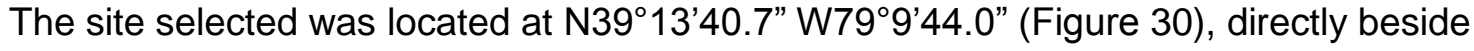
the truck ramp off of U.S. 48. The site had a slope of $15.4 \%$, elevation of $1837 \mathrm{ft}(560 \mathrm{~m})$, and slope exposure of $170^{\circ} \mathrm{SE}$. The site location can be seen in Figure 6 . The plot layout is displayed in Figure 31 and was named $\mathrm{CH}-3$. There are two replications with 24 subplots. Please see Table 20 for treatments. The seed mixtures used were WVDOH Type B (DOH), Developed Type B (TB), and Developed Type C (TC). Treatments either have topsoil (TS) or no topsoil (NTS). The mulch used was straw (S) or a HECP (HP). The subplots were $5.7 \mathrm{ft} \times 6 \mathrm{ft}$ $(1.75 \mathrm{~m} \times 1.83 \mathrm{~m})$. Randomization was conducted the same as in Section 4.3.1.1 and can be seen in Figure 31.

Table 20: Objective 3 Plot Design

\begin{tabular}{lcccccccccccc}
\hline Treatment & $\mathbf{1}$ & $\mathbf{2}$ & $\mathbf{3}$ & $\mathbf{4}$ & $\mathbf{5}$ & $\mathbf{6}$ & $\mathbf{7}$ & $\mathbf{8}$ & $\mathbf{9}$ & $\mathbf{1 0}$ & $\mathbf{1 1}$ & $\mathbf{1 2}$ \\
\hline Seed Mix & DOH & DOH & DOH & DOH & TB & TB & TB & TB & TC & TC & TC & TC \\
Topsoil & TS & TS & NTS & NTS & TS & TS & NTS & NTS & TS & TS & NTS & NTS \\
Mulch & HP & S & HP & S & HP & S & HP & S & HP & S & HP & S \\
\hline
\end{tabular}

Note:

1. $\mathrm{DOH}=\mathrm{WVDOH}$ Type $\mathrm{B}$ (Medians, shoulders, waterways, and mowable areas)

2. $\mathrm{TB}=$ Developed Type $\mathrm{B}$ (Warm Season)

3. TC= Developed Type C (Cool Season)

4. $\mathrm{TS}=$ Topsoil 
5. NTS $=$ No Topsoil

6. $\mathrm{HP}=$ Hydraulic Erosion Control Products

7. $\mathrm{S}=$ Straw Mulch

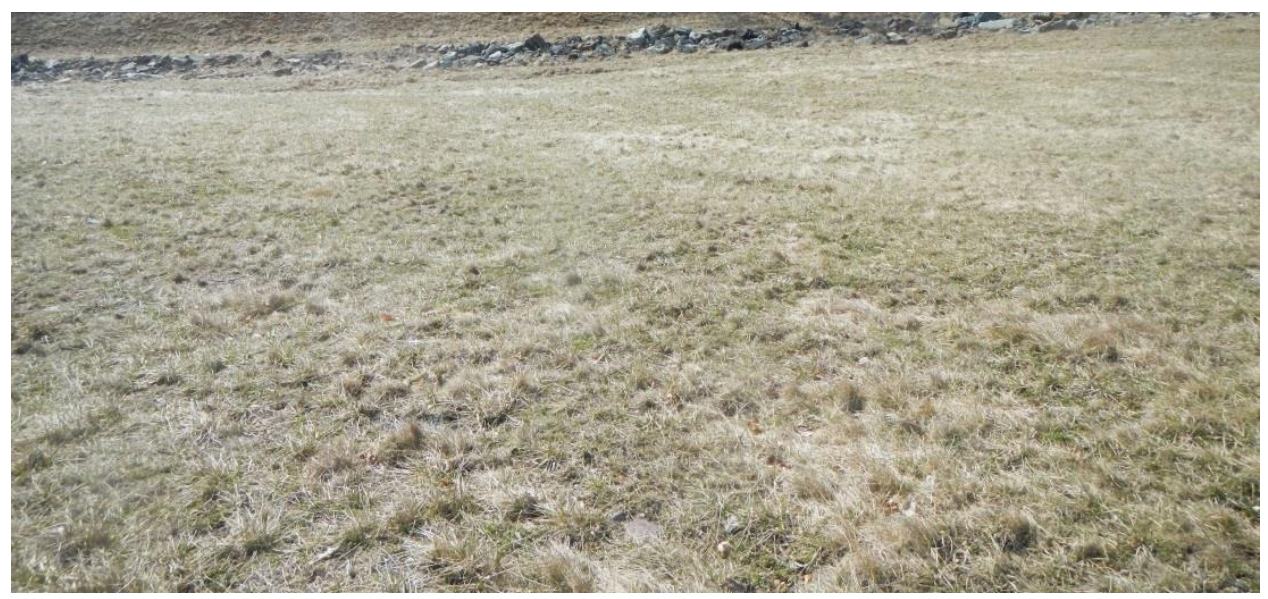

Figure 30: CH-3 Study Site

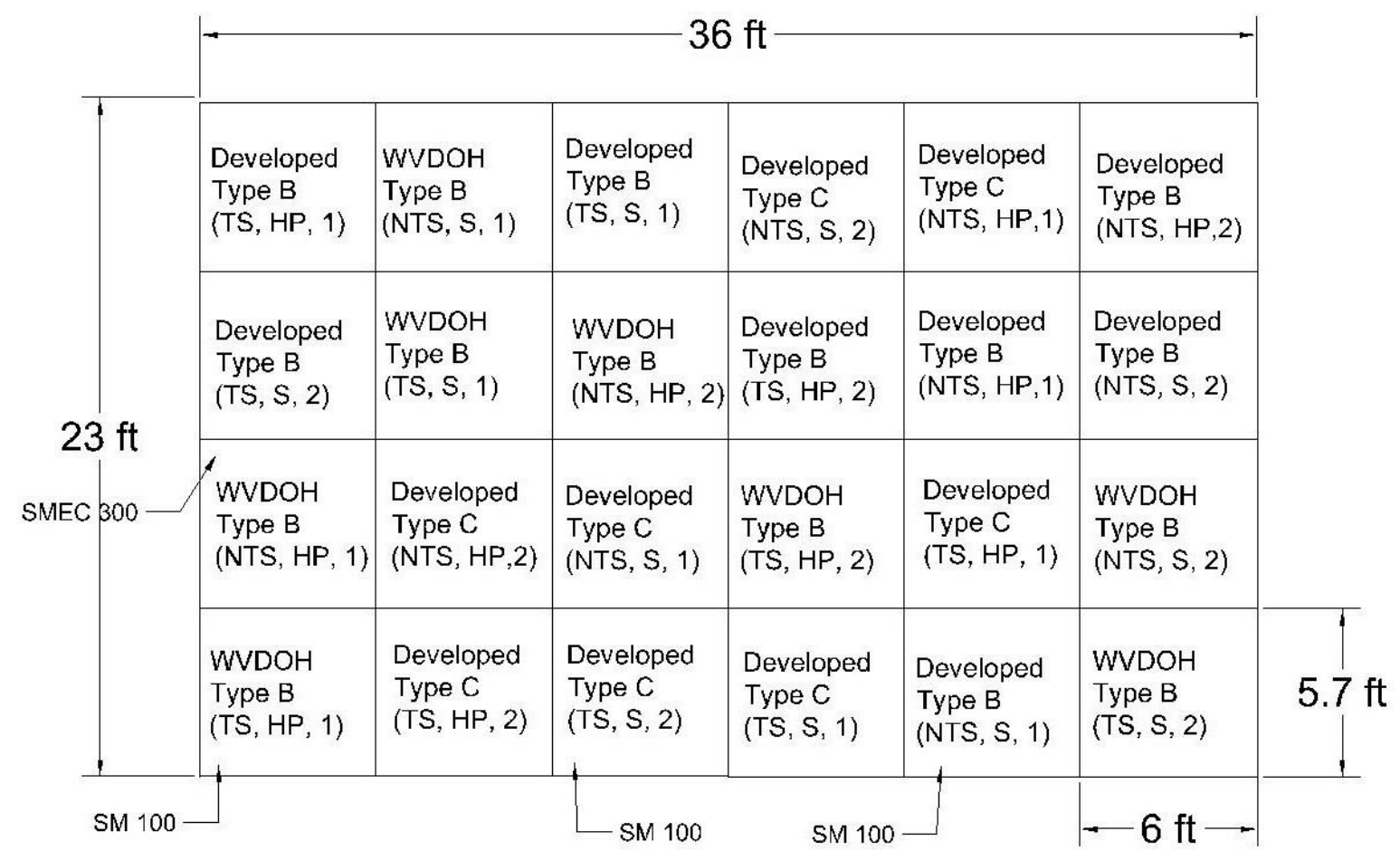

Figure 31: CH-3 Subplot Layout (TS= topsoil, NTS= no topsoil, HP= hydraulic erosion control product, $\mathrm{S}=$ straw)

\section{Site Preparation}

On April 17, 2015, black plastic (Figue 32) was installed to kill the existing vegetation to prepare the site for treatment application in May. Final vegetation removal was performed on May 15, 2015 and black plastic was re-installed until seedbed preparation and planting 
occurred. The fence installation was the same as in Section 4.3.1.1 and can be seen in Figure 33.

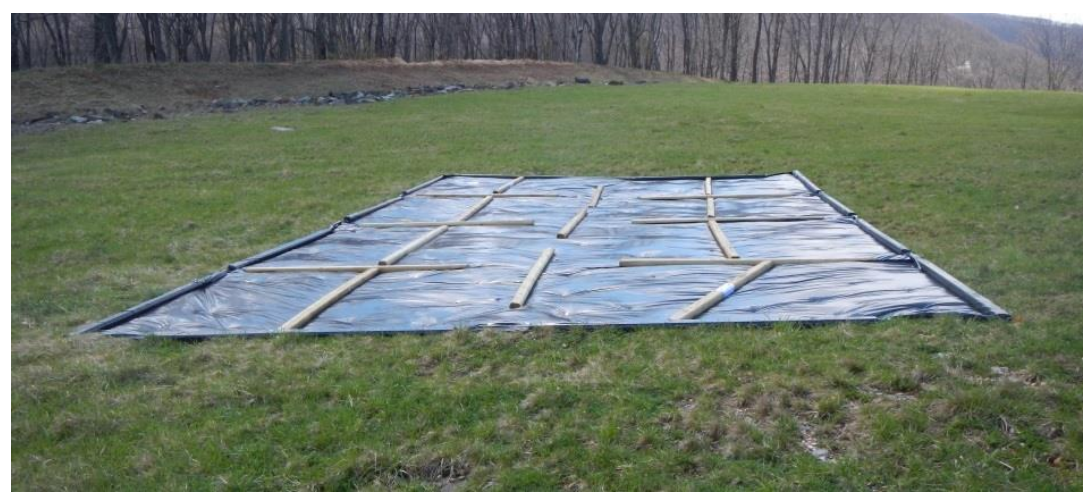

Figure 32: CH-3 Vegetation Removal

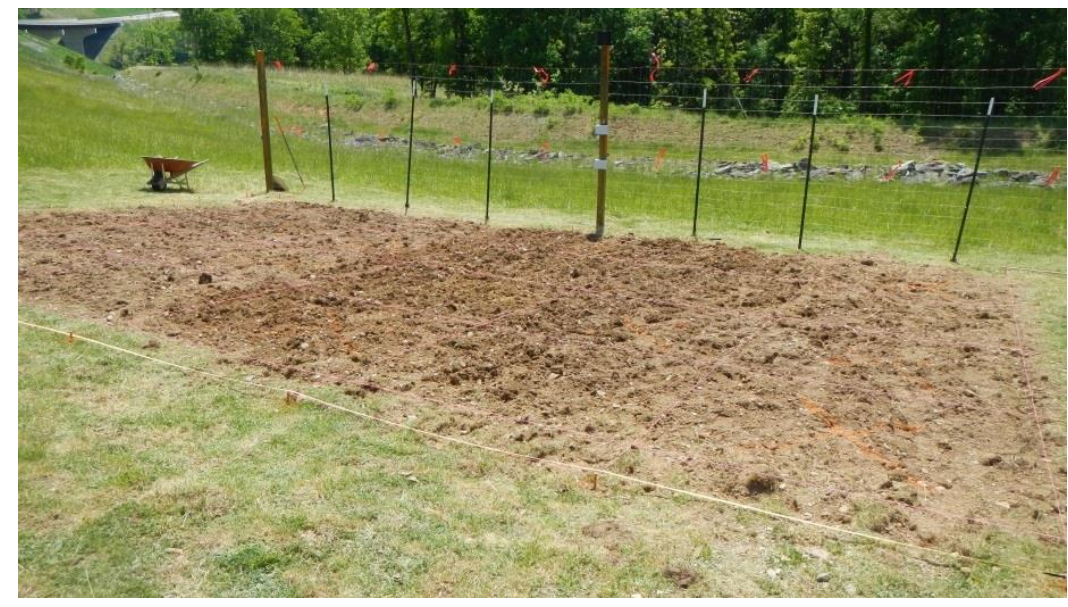

Figure 33: CH-3 Fence Installation

Initial seedbed preparation was conducted the same as in Section 4.3.1.1. The difference was the installation of topsoil in the subplots requiring it in Figure 31. Approximately $0.25 \mathrm{ft}(0.076 \mathrm{~m})$ of the existing base material was removed and placed on a neighboring subplot that was designated as a no-topsoil treatment. The next step was to apply $0.5 \mathrm{ft}(0.15$ $\mathrm{m}$ ) of topsoil and level out the completed subplots, seen in Figure 34 and 35 . Then a $300 \mathrm{lb}$ (136 kg) Sakai PF150 plate compactor (Figure 35) was used to re-compact the entire site to mimic earthwork construction. Finally, the soil surface was scarified using a steel garden rake to a depth of 0.5-1 in. (0.01-0.03 m). The prepared seedbed can be seen in Figure 36. 


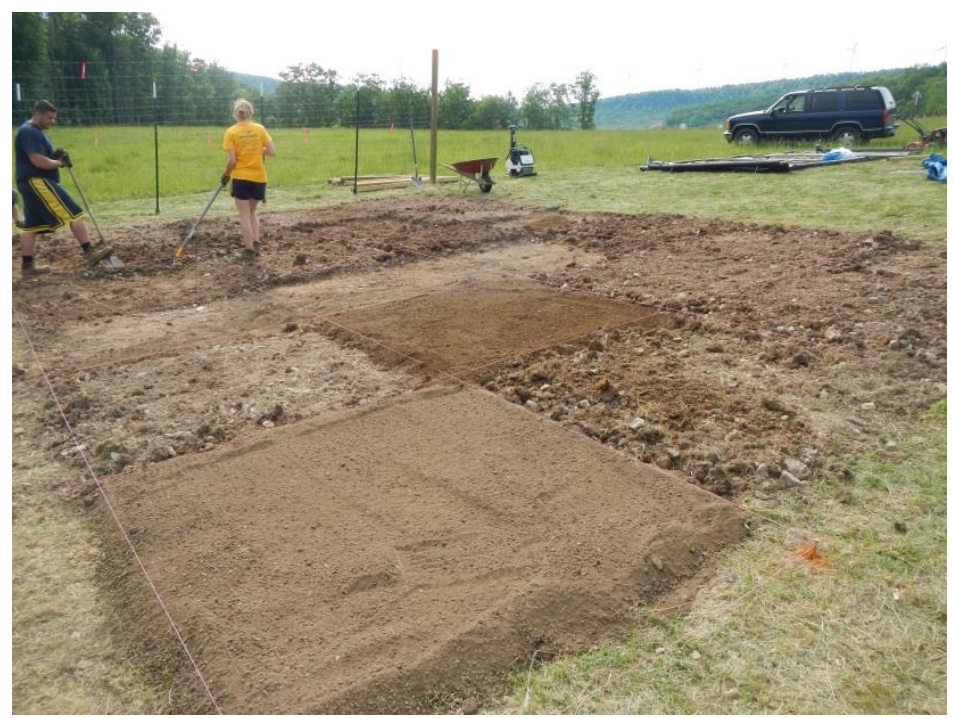

Figure 34: CH-3 Seedbed Preparation

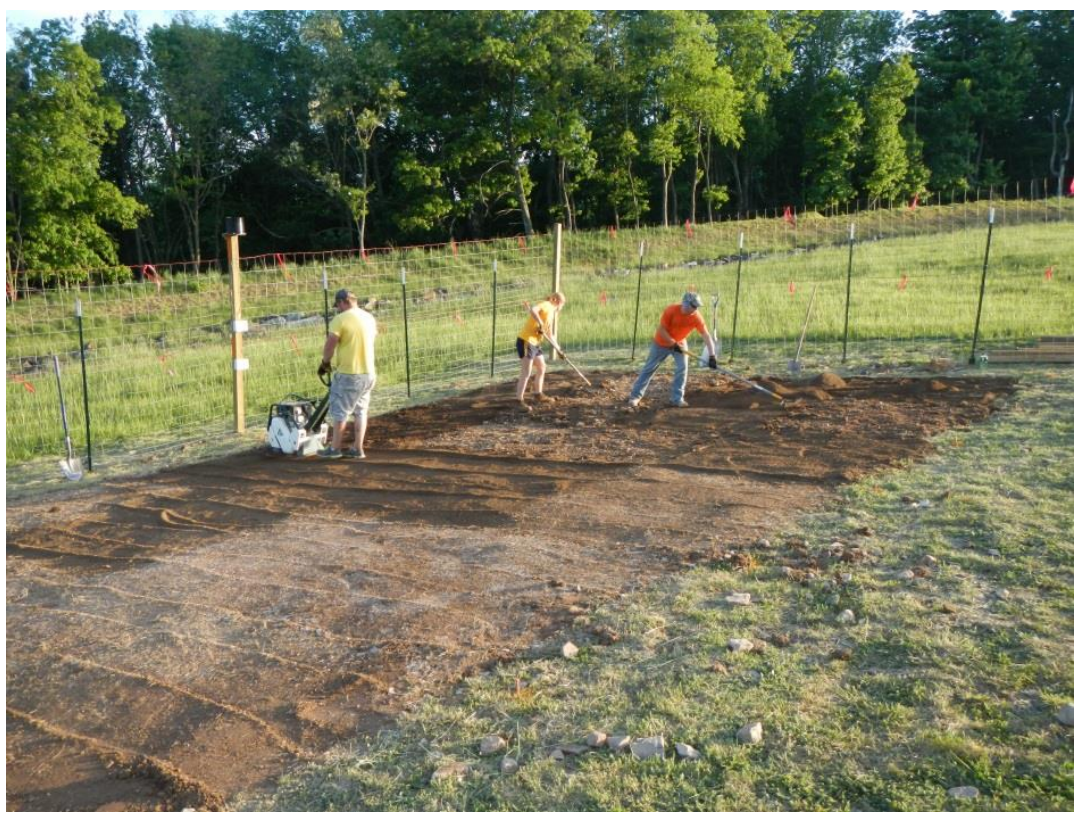

Figure 35: $\mathrm{CH}-3$ Compaction 


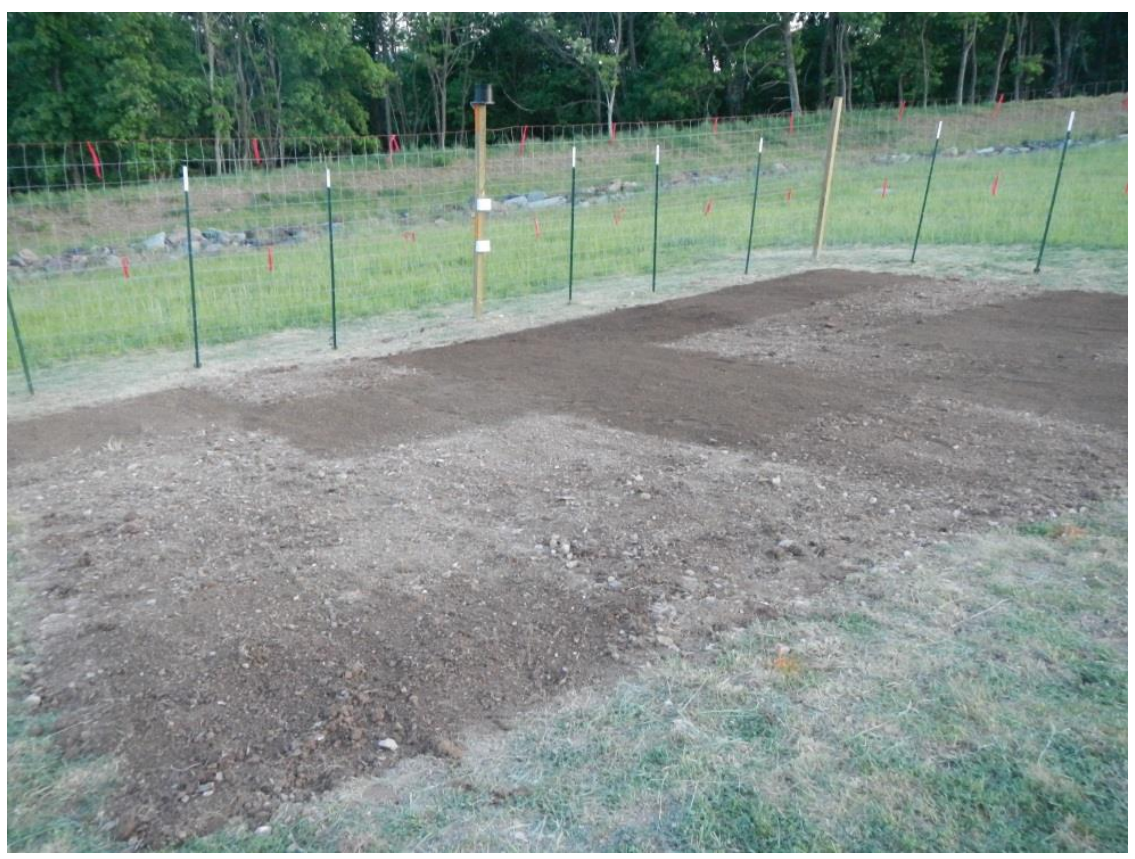

Figure 36: CH-3 Seedbed

\section{Equipment Installation}

After seedbed preparation, a grid system and research equipment were installed. The grid system was composed of 248 in. $(0.2 \mathrm{~m})$ landscape stakes and twisted mason line. The grid system was laid out in accordance with the suplot design in Figure 31. The installation of the grid system was the same as in Section 4.3.1.1 and can be seen in Figure 37.

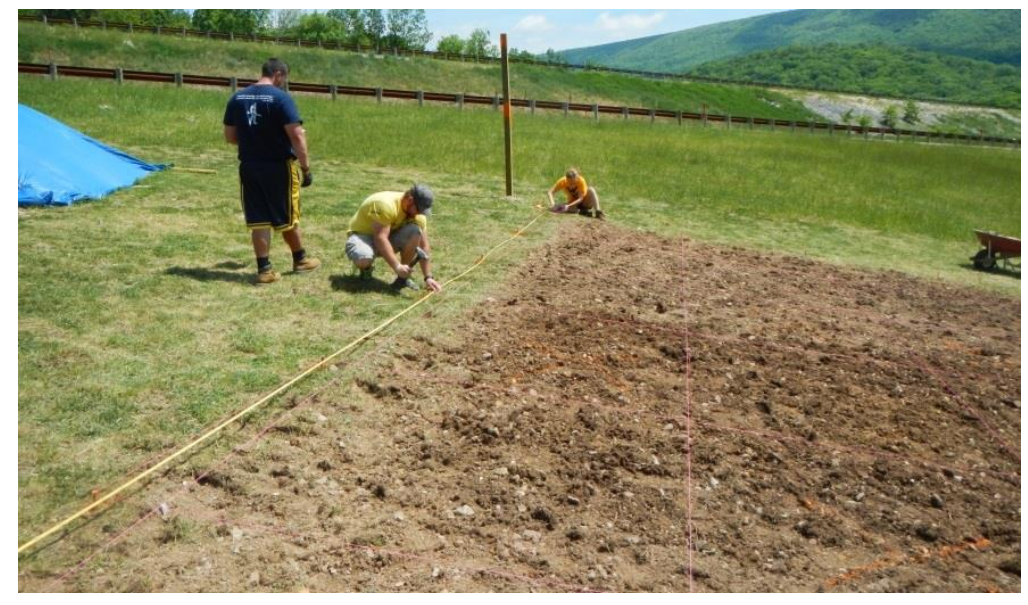

Figure 37: CH-3 Grid Installation

The research equipment installed entailed a WatchDog Tipping Bucket Rain Gauge, one WaterScout SMEC 300, three WaterScout SM 100 Soil Moisture Sensors, one WatchDog 1400 
Micro Station, and one WatchDog 1425 Micro Station. Equipment details and use are specified in Section 4.3.2. The WatchDog Tipping Bucket Rain Gauge and WatchDog 1425 Micro Station were installed the same manor as in Section 4.3.1.1.

A WaterScout SM 100 was installed into subplot "Developed Type B (NTS, S, 1)" seen in Figure 31 . The installation was conducted by placing the sensor $0.5 \mathrm{ft}(0.15 \mathrm{~m})$ into the soil media. The sensor was placed in the soil perpendicular to the surface and firmly pressed into the soil face after a slot was made with a knife. The slot for the sensor was necessary to avoid breaking the SM 100, seen in Figure 38, 39, and 40. Conduit installation and equipment launching was conducted in accordance to Section 4.3.1.1.

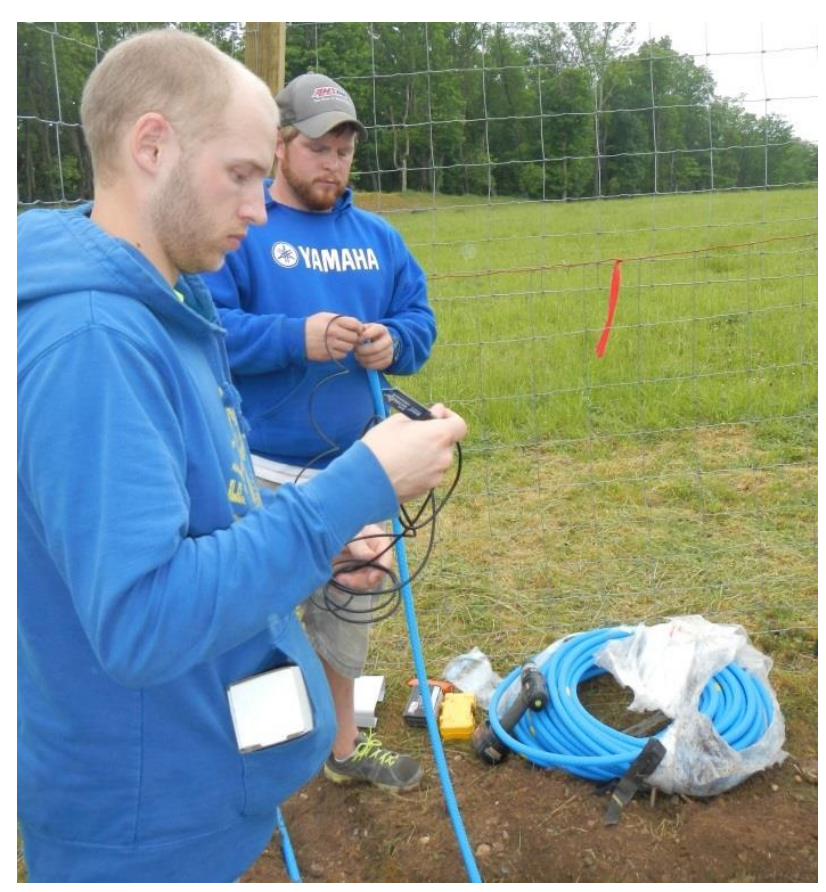

Figure 38: CH-3 Conduit Installation 


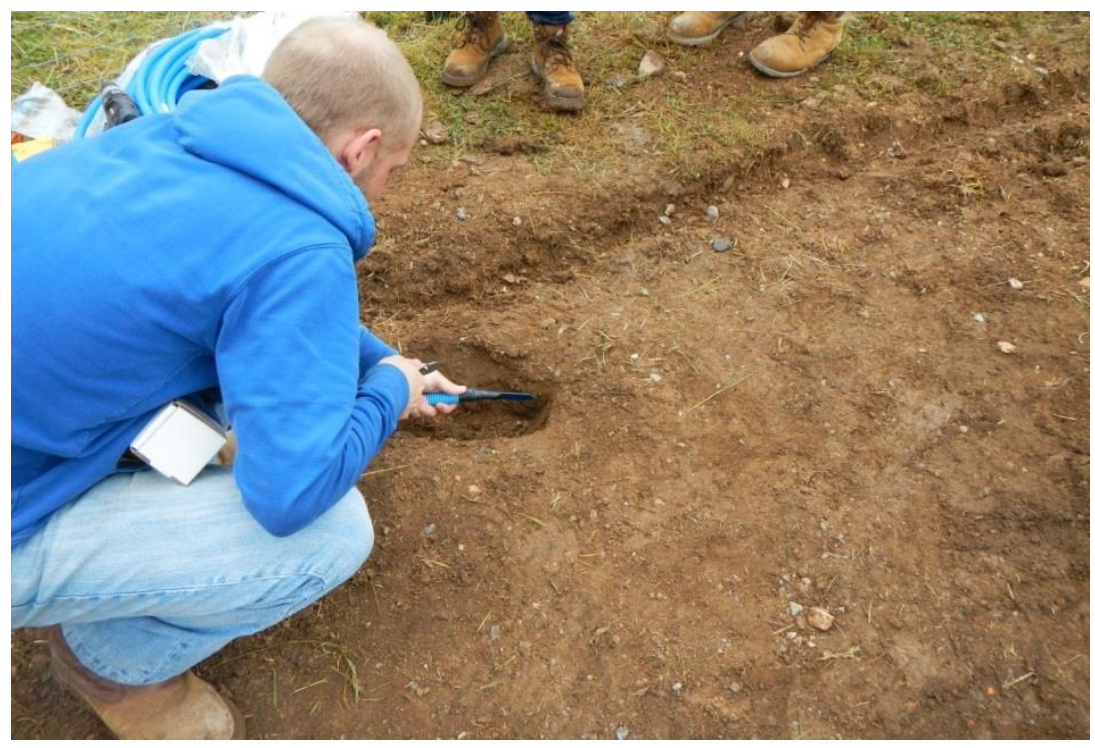

Figure 39: SMEC 300 Installation

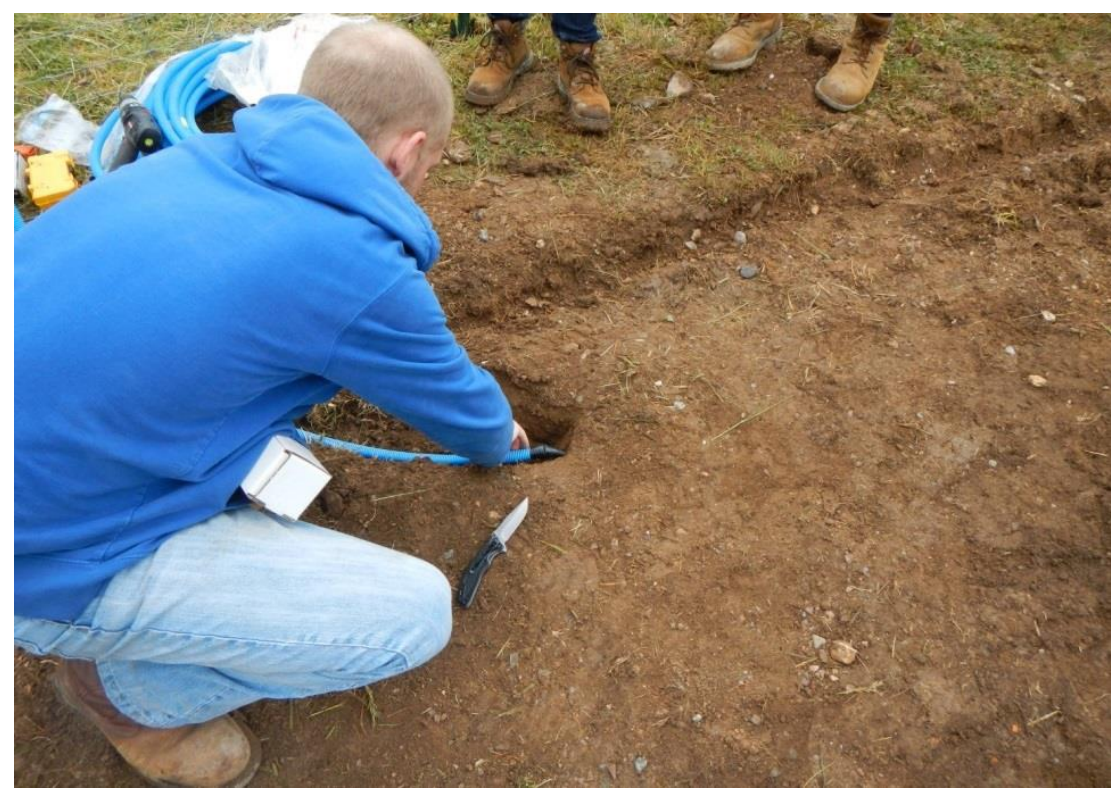

Figure 40: SMEC 300 Installation Completion

A WaterScout SMEC 300 and two SM 100's were also installed into the research site.

The SMEC 300 was installed in subplot "WVDOH Type B (NTS, HP, 1)" and plugged into channel D of the WatchDog 1400 Micro Station. One SM 100 was installed in subplot "WVDOH Type B (TS, HP,1)" and plugged into channel A. The other SM 100 was installed in subplot “Developed Type C (TS, S, 2)" and plugged into channel B of the 1400 Micro Station. The installation was conducted the same as above, except the WatchDog 1400 Micro Station 
enclosure was mounted $1 \mathrm{ft}(0.3 \mathrm{~m})$ off of the ground on a corner wood post. Instrument installation location can be seen in Figure 31.

\section{Fertilizer and Seed Application}

The next step was to apply fertilizer and seed to each subplot. Fertilizer application recommendation for $\mathrm{CH}-3$ was determined from the soil tests seen in Table 21 displayed as "CH-3" for the subsoil and "CH-3 Topsoil" for the topsoil. Fertilizer recommendation was 50-40$105 \mathrm{lb} / \mathrm{acre}$ for the subsoil and 91.5-78.4-108.9 lb/acre for the topsoil. The fertilizer used was a 10-10-10 with a grand total of $4.75 \mathrm{lb}(2.16 \mathrm{~kg})$ for the non-topsoil subplots site displayed in Table 21. Table 21 displays two topsoil quantities because an error occurred with topsoil fertilizer quantities. $\mathrm{CH}-3$ Topsoil 1 was applied during the planting stage and was only $2 \%$ of the recommended quantity for the installed topsoil. To fix the error, $\mathrm{CH}-3$ Topsoil 2 fertilizer quantity was installed on June 24,2015 on top of the mulch. The quantities were measured in conjunction to Section 4.3.1.1 methods.

Table 21: Fertilizer Application Quantity

\begin{tabular}{|c|c|c|c|c|c|c|c|}
\hline \multirow{2}{*}{$\begin{array}{l}\text { Site } \\
\text { Location }\end{array}$} & \multirow{2}{*}{$\begin{array}{c}\text { Experimental } \\
\text { Area }\end{array}$} & \multirow{2}{*}{$\begin{array}{c}\text { Experimental } \\
\text { Area }\end{array}$} & \multicolumn{3}{|c|}{ Soil Test Results } & \multirow{2}{*}{$\begin{array}{c}\text { Ratio } \\
\text { Available } \\
\end{array}$} & \multirow{2}{*}{$\begin{array}{c}\text { Quantity } \\
\text { Applied } \\
\mathrm{N}\end{array}$} \\
\hline & & & $\mathrm{N}$ & $\mathrm{P}_{2} \mathrm{O}_{5}$ & $\mathrm{~K}_{2} \mathrm{O}$ & & \\
\hline Units & $\mathrm{ft}^{2}$ & acre & & lb/acre & & & $\mathrm{lb}$ \\
\hline $\mathrm{CH}-3$ & 414 & 0.0095 & 50 & 40 & 105 & $\begin{array}{c}10-10- \\
10\end{array}$ & 4.75 \\
\hline $\begin{array}{c}\mathrm{CH}-3 \\
\text { Topsoil } 1\end{array}$ & 414 & 0.0095 & 91.5 & 78.4 & 108.9 & $\begin{array}{c}10-10- \\
10\end{array}$ & 0.20 \\
\hline $\begin{array}{c}\text { CH-3 } \\
\text { Topsoil } 2\end{array}$ & 414 & 0.0095 & 91.5 & 78.4 & 108.9 & $\begin{array}{c}10-10- \\
10\end{array}$ & 8.50 \\
\hline
\end{tabular}

Seed application began by determining the correct application amount for each subplot. Seed mixture application quantities for seed and inoculant:

- WVDOH Type B

○ Application amount (0.576 lbs.) (8- 32.66 gram bags) $(+/-0.030 \mathrm{~g})$

- Inoculant $(0.245 \mathrm{~g})(+/-0.030 \mathrm{~g})$

- Developed Type B

○ Application amount (0.453 lbs.) (8- 25.68 gram bags) (+/- $0.030 \mathrm{~g})$ 
○ Inoculant $(0.193 \mathrm{~g})(+/-0.030 \mathrm{~g})$

- Developed Type C

- Application amount (0.348 lbs.) (8- 19.73 gram bags) $(+/-0.030 \mathrm{~g})$

$\circ$ Inoculant $(0.148 \mathrm{~g})(+/-0.030 \mathrm{~g})$

With eight subplots per seed mixture, the application amount was divided into eight equal parts, weighed, mixed with the correct inoculant, and stored in a paper bag until planting. The measuring procedure for the seed was the same as in Section 4.3.1.1. Seeding application can be seen in Figure 41. Once the seed mixtures were applied, a steel garden rake was used to scuff the surface to promote seed to soil contact. After the seed mixtures were planted, the black tarp was re-applied to the plot area until the HECP could be applied the next morning.

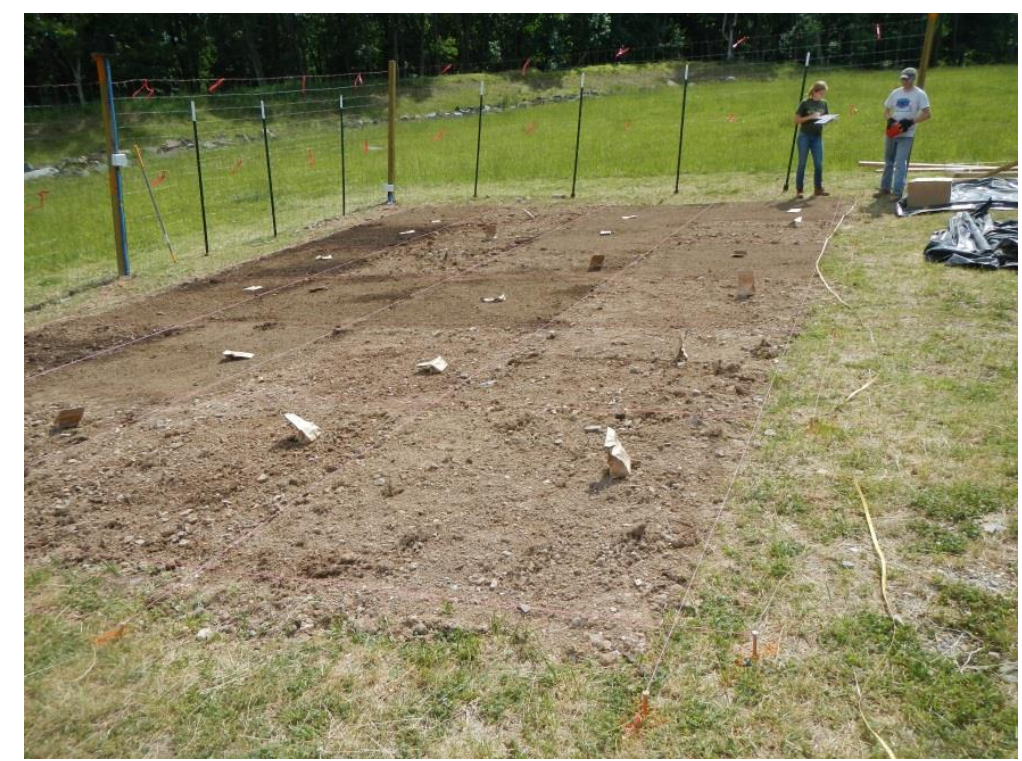

Figure 41: Seed Mixture Application

\section{HECP Application}

On May 28, 2015, Arnold's Custom Seeding, LLC (ACS) arrived on site to begin HECP and straw tackifier application. The HECP application and product specifics are the same as in Section 4.3.1.1. The straw tackifier used was Tornado Tack ${ }^{\mathrm{TM}}$ ST-1000 by Profile Products, LLC at an application rate of $500 \mathrm{lb} / \mathrm{ac}(560 \mathrm{~kg} / \mathrm{ha})$. Approximately $38 \mathrm{lb}(17 \mathrm{~kg})$ of straw was applied over twelve subplots before tackifier application. Profile Products, LLC again attended application of their product to verify proper application rate and technique was performed 
(Profile Products LLC. 2015a and 2015b). Application of ProMatrix can be seen in Figure 42 and application of Tornado Tack can be seen in Figure 43 by a FiNN T170 Hydro Seeder. Excess product was mixed in the hydro seeder to ensure proper mixing of the product and application abilities of the machine.

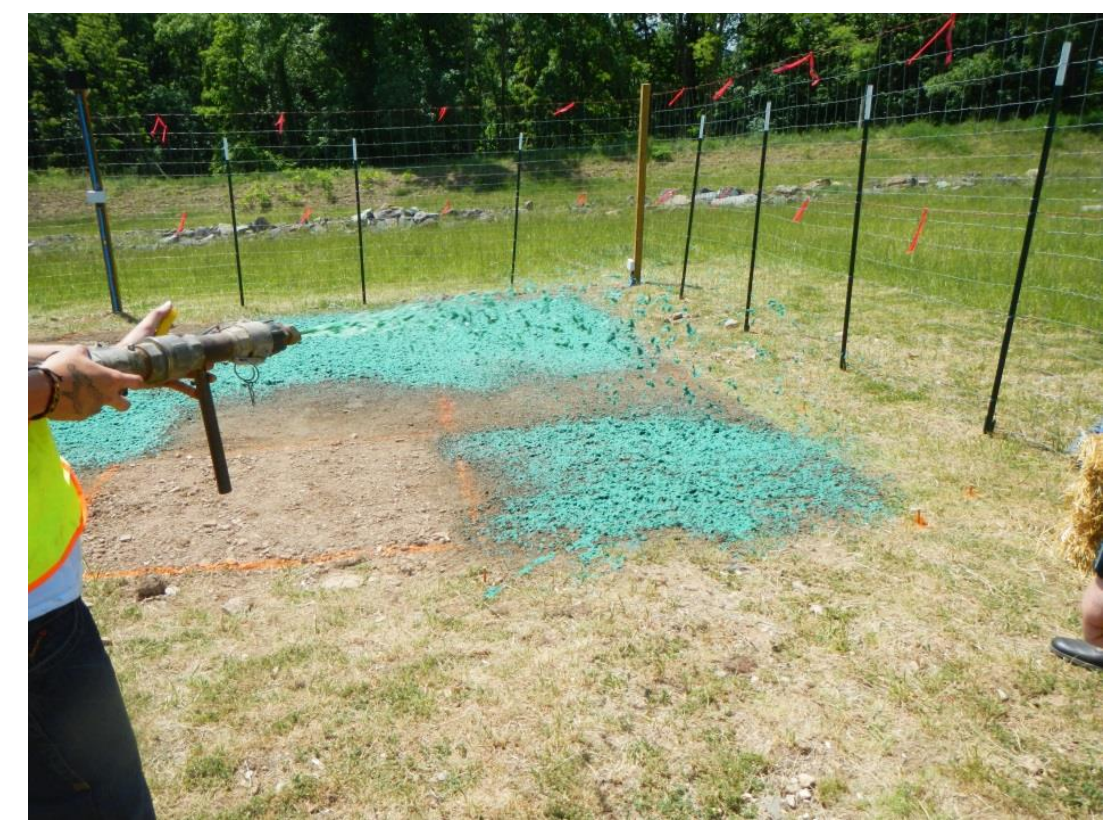

Figure 42: CH-3 HECP Application

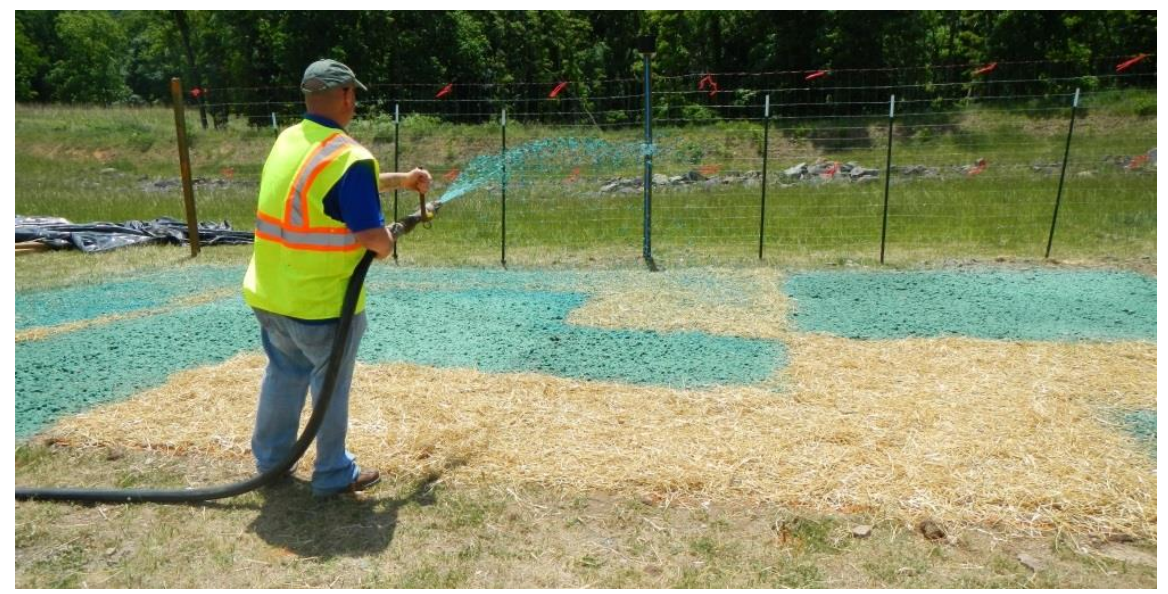

Figure 43: CH-3 Straw Tackifier Application

Tornado Tack is a straw tackifier composed of wood cellulose, organic biopolymers, interlocking fibers, and porous ceramic particles. The recommended installation is $65-70 \mathrm{lb} / 100$ gallons $(29-31 \mathrm{~kg} / 379 \mathrm{~L})$ of water mixed in a hydroseeding tank (Profile Products LLC. $2015 \mathrm{~b}$ ). 
Before tackifier application, the bare straw can be seen in Figure 44. The completed site with tackifier on the straw can be seen in Figure 45.

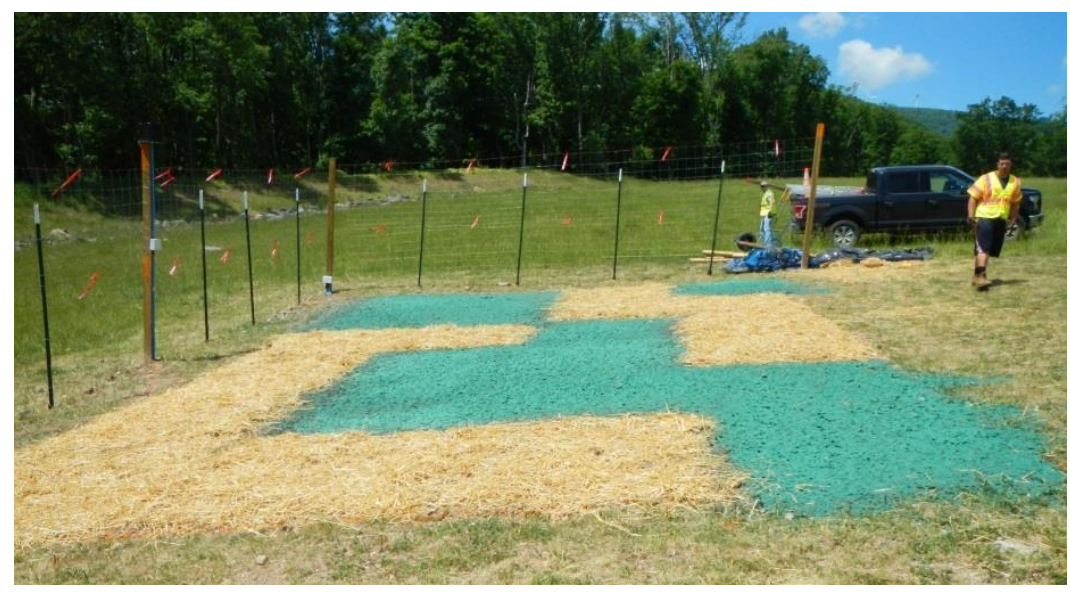

Figure 44: CH-3 HECP and Straw

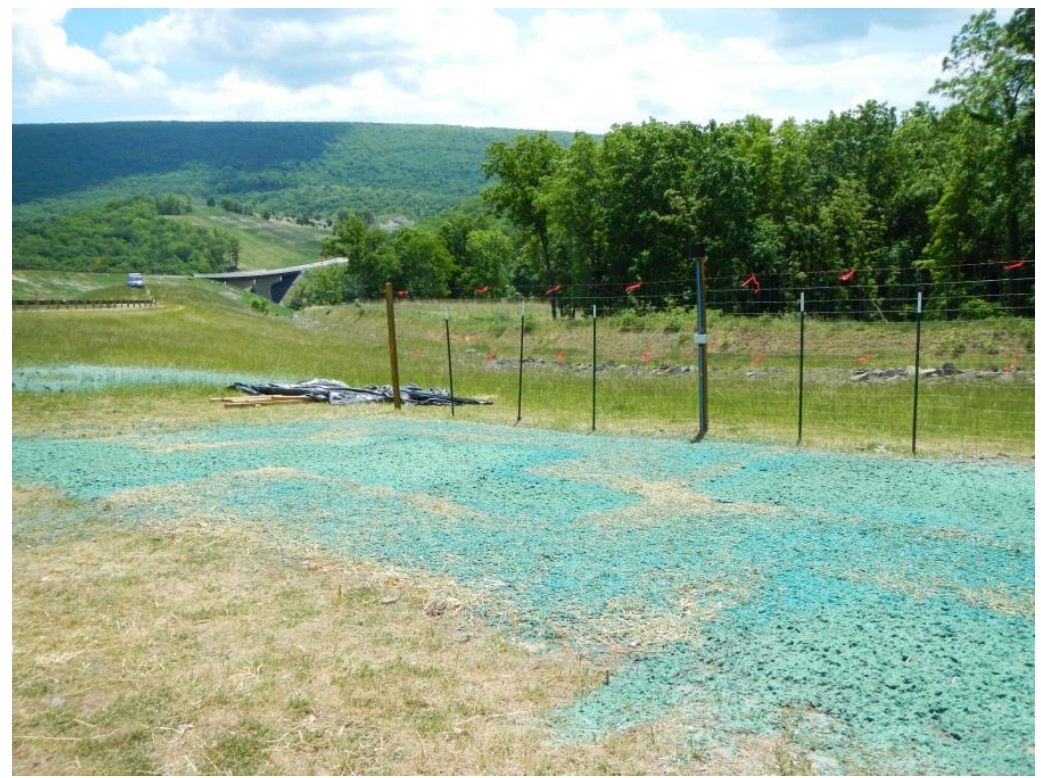

Figure 45: CH-3 HECP and Tackifier

\subsubsection{Objective 4: Examining soil media and amendment products}

Site Selection and Layout

Objective 4 evaluated the performance of soil amendments in comparison to topsoil and no topsoil. The seed mixture utilized was Developed Type A and the site was located at N3907'55.6”, W7858'55.3", directly beside county road 220/8 (Fish Pond Road). The study site was named $\mathrm{CH}-1 \mathrm{~B}$ and can be seen in Figure 7. The site location can be seen in Figure 6. 
The site had a slope of $4.2 \%$, elevation of $840 \mathrm{ft}(256 \mathrm{~m})$, and slope exposure of $40^{\circ} \mathrm{NE}$. The subplots were $5.4 \mathrm{ft} \times 5.7 \mathrm{ft}(1.66 \mathrm{~m} \times 1.75 \mathrm{~m})$. Each subplot was replicated three times, totalling 12 subplots. Plot layout can be seen in Figure 46. The suplots examined vegetative establishment and growth in topsoil, no topsoil, Biotic Earth, and ProGanics. Randomization of the subplots was conducted the same as in Section 4.3.1.1 and can be seen in Figure 46.

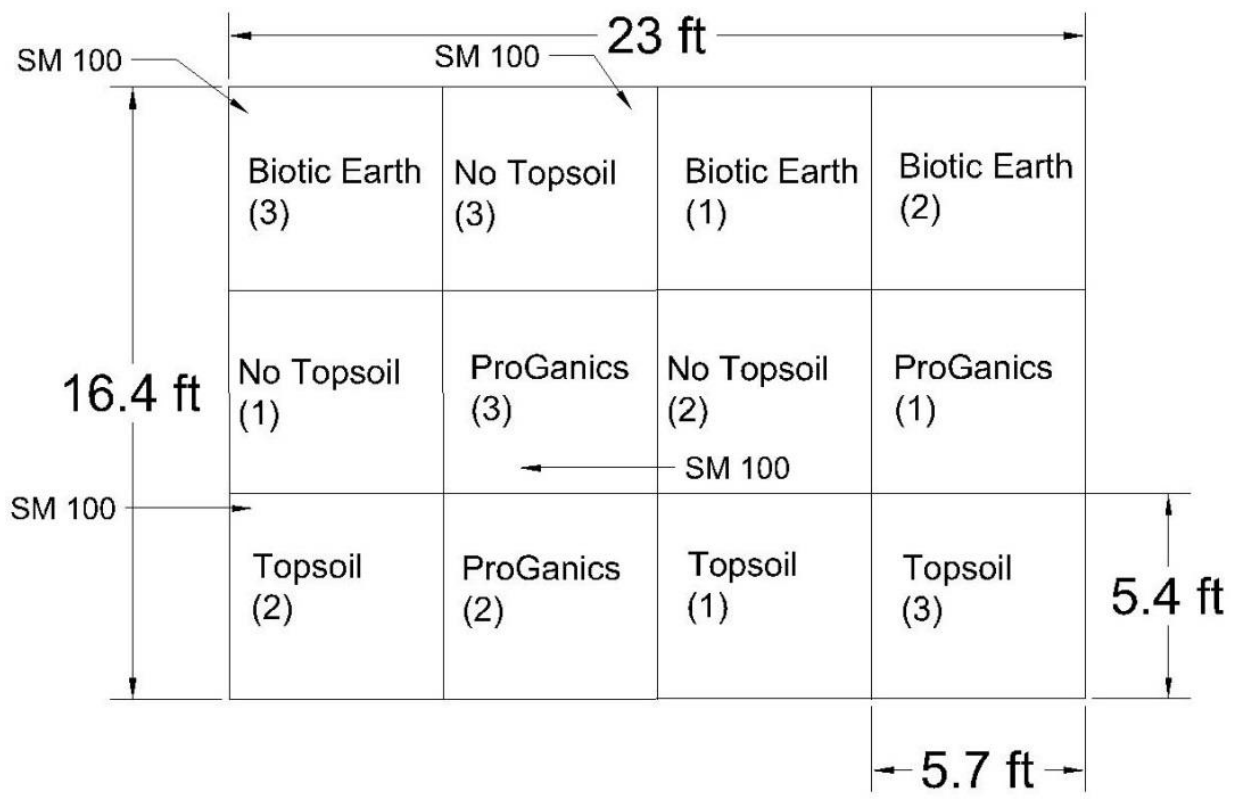

Figure 46: CH-1B Subplot Layout

\section{Site Preparation}

Site preparation began with the installation of black plastic (Figue 47) and fence (Figure 48) in the same manner as Section 4.3.1.1. Final vegetation removal and seedbed preparation were also conducted the same as in Section 4.3.1.1. The difference was the application of topsoil in the designated subplots displayed in Figure 48. Approxiametly $0.25 \mathrm{ft}(0.076 \mathrm{~m})$ of the existing base material was removed and placed on an adjacent subplot that was designated as a no-topsoil treatment. Then, $0.5 \mathrm{ft}(0.15 \mathrm{~m})$ of topsoil was applied and leveled, seen in Figure 48 and 49. A $300 \mathrm{lb}(136 \mathrm{~kg})$ Sakai PF150 plate compactor was then used to re-compact the entire site to mimic earthwork construction. Finally, the soil surface was scarified using a steel garden rake to a depth of $0.5-1 \mathrm{in} .(0.01-0.03 \mathrm{~m})$. The prepared seedbed can be seen in Figure 50. 


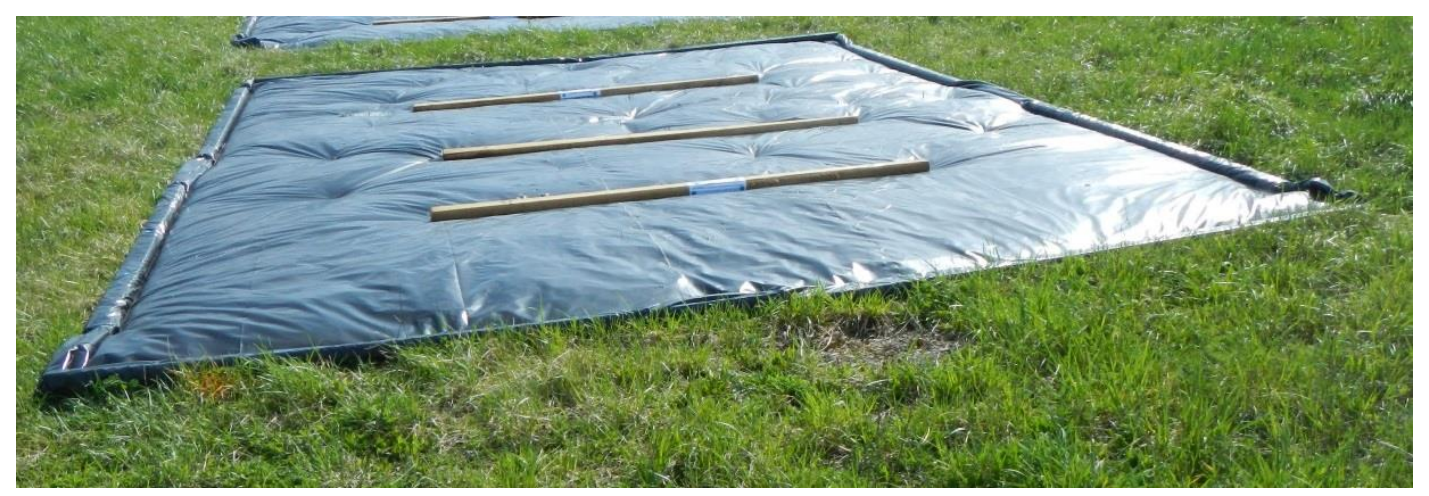

Figure 47: $\mathrm{CH}-1 \mathrm{~B}$ Vegetation Removal

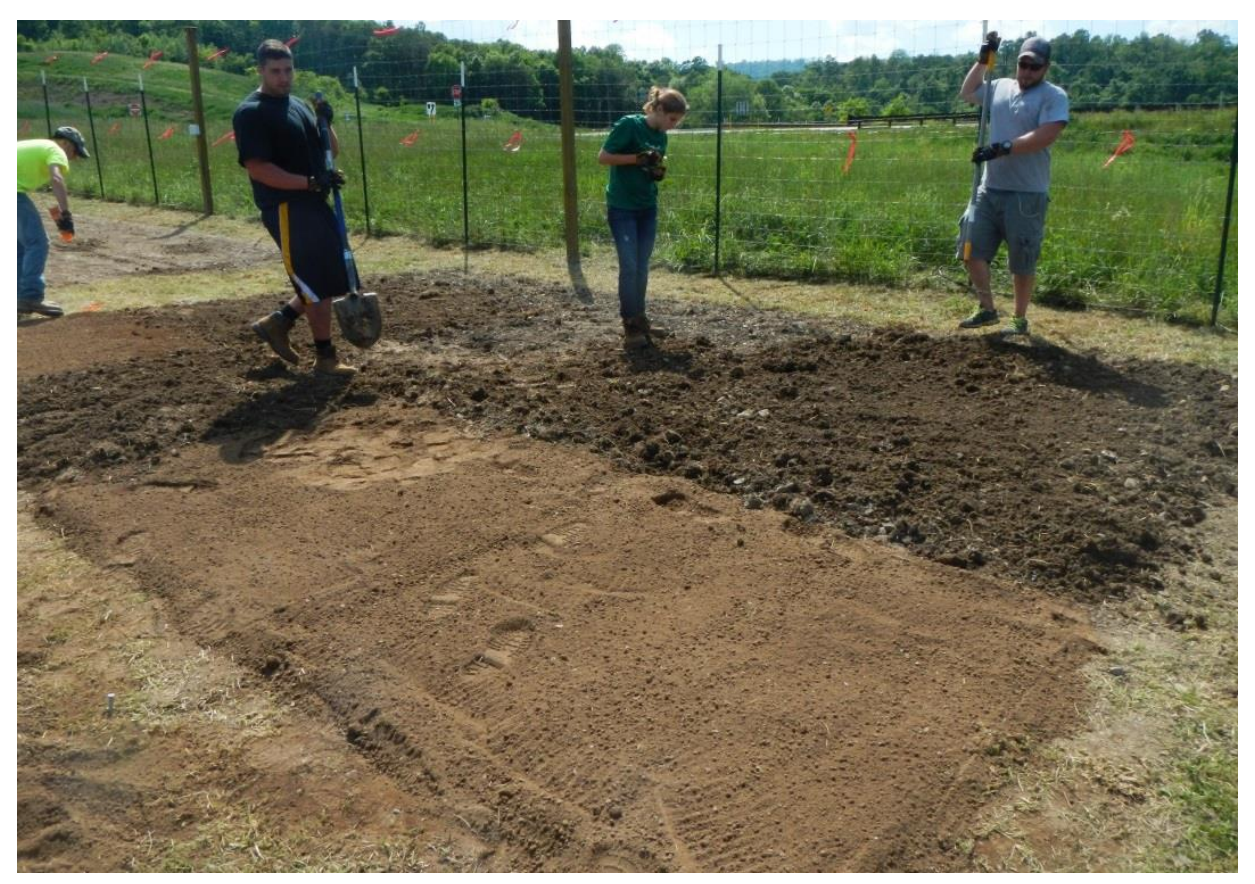

Figure 48: CH-1B Seedbed Preparation 


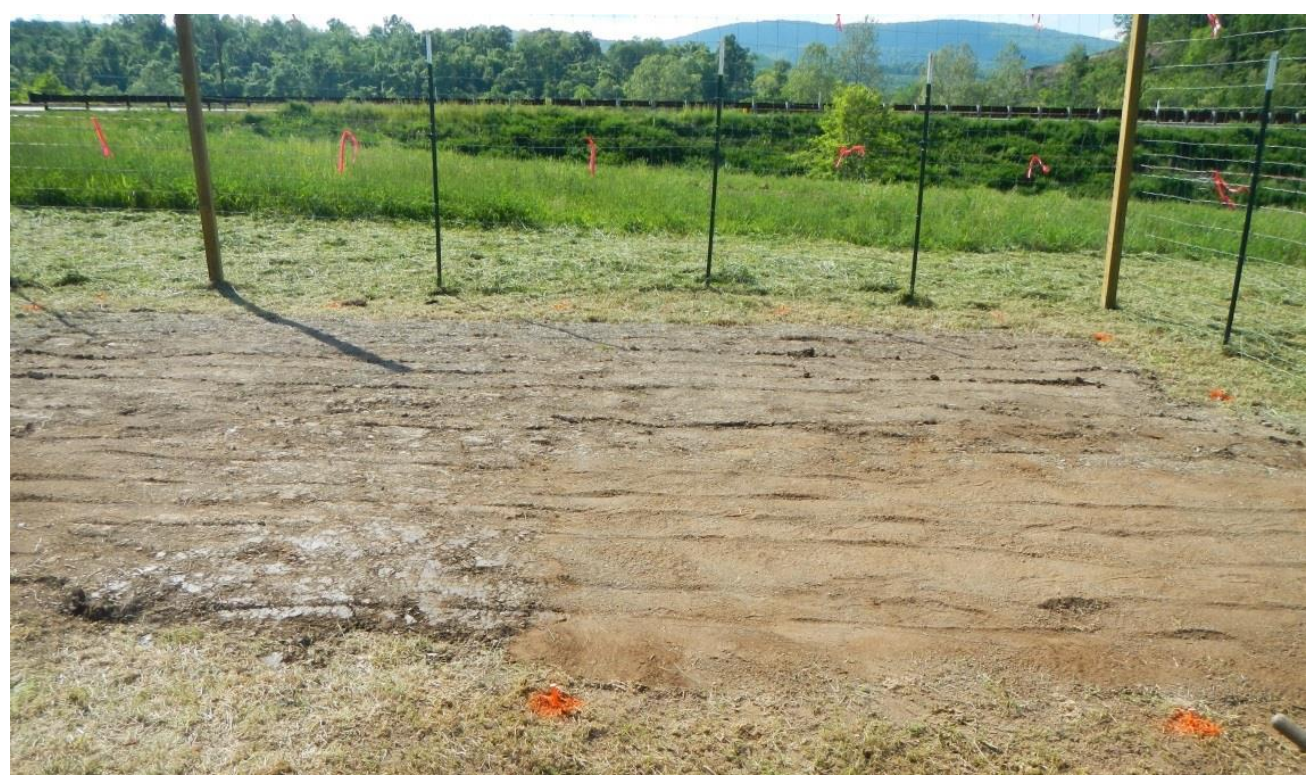

Figure 49: $\mathrm{CH}-1 \mathrm{~B}$ Compaction

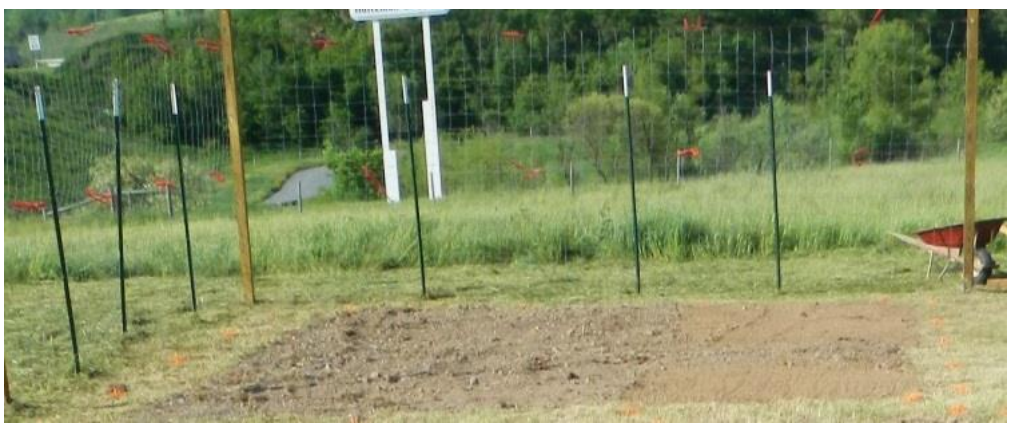

Figure 50: CH-1B Seedbed

\section{Equipment Installation}

Following seedbed preparation, a grid system and research equipment were installed.

The grid system was composed of 188 in. $(0.2 \mathrm{~m})$ landscape stakes and twisted mason line.

The grid system was laid according to the subplot design in Figure 46. The grid installation was the same as in Section 4.3.1.1 and can be seen in Figure 51. 


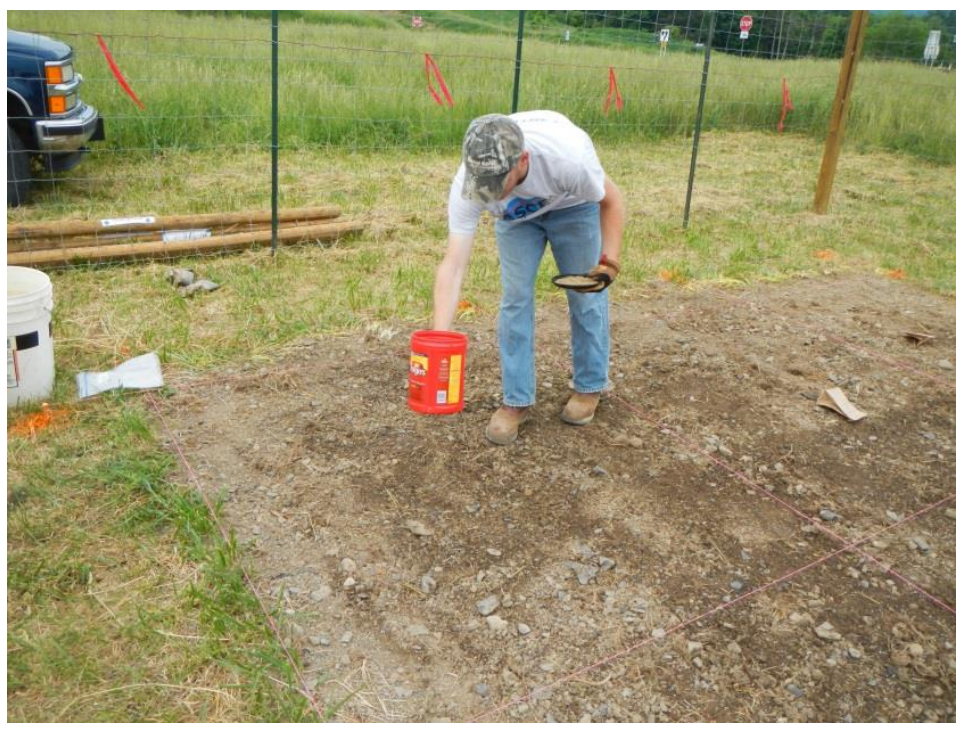

Figure 51: CH-1B Grid Installation and Seeding

The research equipment installed entailed four WaterScout SM 100 Soil Moisture Sensors, and one WatchDog 1400 Micro Station. Equipment details and use are specified in Chapter 4.3.2. A WatchDog Tipping Bucket Rain Gauge and WatchDog 1425 Micro Station were mounted for $\mathrm{CH}-1 \mathrm{~A}$, directly beside $\mathrm{CH}-1 \mathrm{~B}$, and the precipitation and temperature data from that equipment was used for both sites.

One WaterScout SM 100 was installed into subplot "Topsoil (2)", "Biotic Earth (3)", "ProGanics (3)", and "No Topsoil (3)" seen in Figure 46. The installation was conducted by placing the sensors $0.5 \mathrm{ft}(0.15 \mathrm{~m})$ into the soil media. The sensors were placed in the soil perpendicular to the surface and firmly pressed into the soil face after a slot was made with a knife. The slot for the sensor was necessary to avoid breaking the SM 100. CARLON Ent. Conduit was used to run the SM 100 and rain gauge wire through to protect them from potential weathering and vermin damage. The conduit was buried $0.5 \mathrm{ft}(0.15 \mathrm{~m})$ in the soil and ran up and down the post to the Micro Station enclosure from the rain gauge and SM 100. The installation can be seen in Figure 52.

Once the wiring was in place, the SM 100 for "Topsoil (2)" was plugged into channel A, "Biotic Earth (3)" into channel B, "ProGanics (3)" into channel C, and "No Topsoil (3)" into 
channel D. The WatchDog 1400 Micro Station was then plugged into a laptop and launched with SpecWare 9 Professional. All the equipment was initially tested in the laboratory to verify calibration was correct. The field setup simply launched the equipment to begin data recording and verified everything was working correctly in the field.

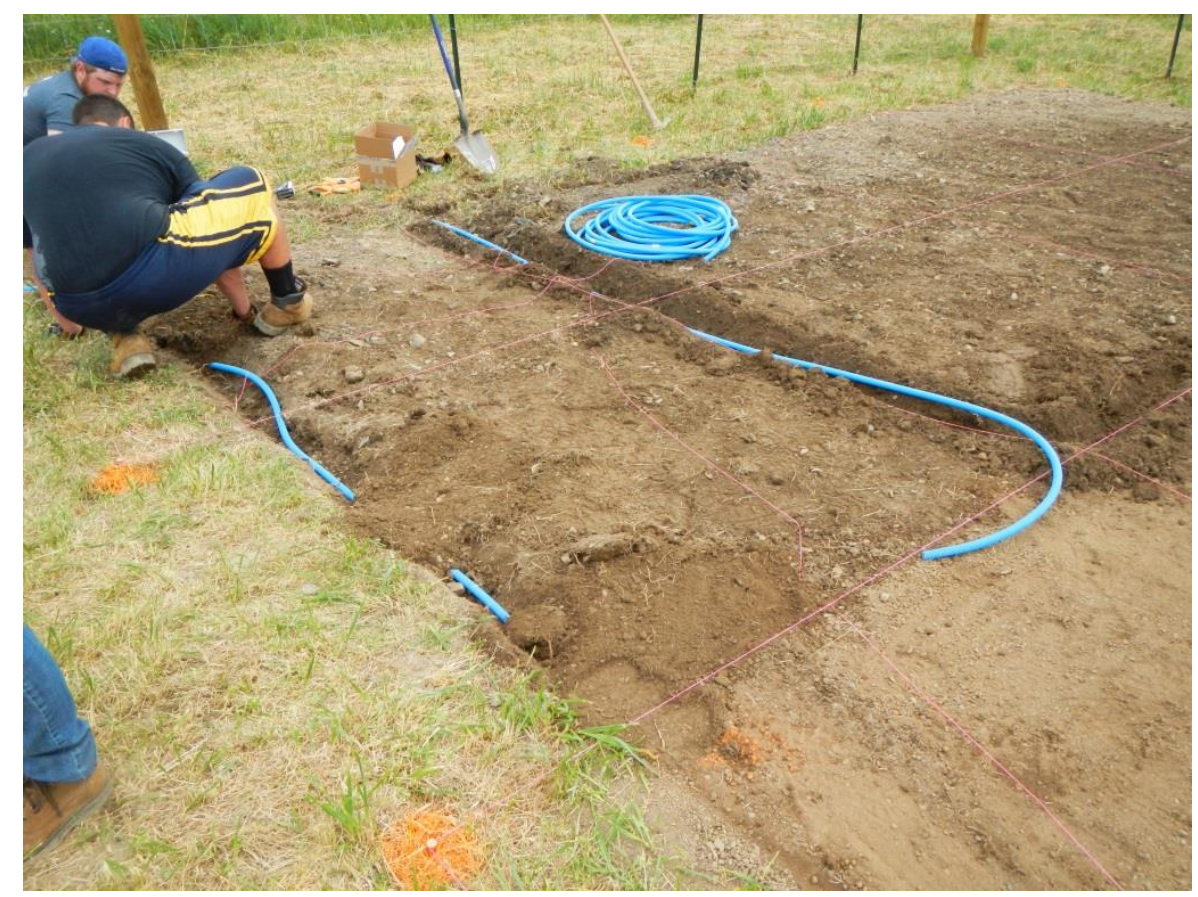

Figure 52: $\mathrm{CH}-1 \mathrm{~B}$ Conduit and SM 100 Installation

\section{Fertilizer and Seed Application}

The next step was to apply fertilizer and seed to each subplot. Fertilizer application recommendation for $\mathrm{CH}-1 \mathrm{~B}$ was determined from the soil test seen in Table 22 displayed as "CH-1B" for the subsoil and "CH-1B Topsoil" for the topsoil. Fertilizer recommendation was 5050-50 lb/ac for the subsoil and 91.5-78.4-108.9 lb/ac for the topsoil. The fertilizer used was a 1010-10 with a total of $3.23 \mathrm{lb}(1.46 \mathrm{~kg})$ for the non-topsoil subplots site displayed in Table 22. Table 22 displays two topsoil quantities because an error occurred with topsoil fertilizer quantities. $\mathrm{CH}-1 \mathrm{~B}$ Topsoil 1 was applied during the planting stage and was only $2 \%$ of the recommended quantity for the installed topsoil. To fix the error, $\mathrm{CH}$-1B Topsoil 2 fertilizer quantity was installed on June 24, 2015 on top of the mulch. The fertilizer quantities were measured in the same manner as Section 4.3.1.1 and application can be seen in Figure 51. 
Table 22: Fertilizer Application

\begin{tabular}{|c|c|c|c|c|c|c|c|}
\hline \multirow{2}{*}{$\begin{array}{l}\text { Site } \\
\text { Location }\end{array}$} & \multirow{2}{*}{$\begin{array}{c}\begin{array}{c}\text { Experimental } \\
\text { Area }\end{array} \\
\end{array}$} & \multirow{2}{*}{$\begin{array}{l}\text { Experimental } \\
\text { Area }\end{array}$} & \multicolumn{3}{|c|}{ Soil Test Results } & \multirow{2}{*}{$\begin{array}{c}\text { Ratio } \\
\text { Available }\end{array}$} & \multirow{2}{*}{$\begin{array}{l}\text { Quantity } \\
\text { Needed } \\
\mathrm{N}\end{array}$} \\
\hline & & & $\bar{N}$ & $\mathrm{P}_{2} \mathrm{O}_{5}$ & $\mathrm{~K}_{2} \mathrm{O}$ & & \\
\hline Units & $\mathrm{ft}^{2}$ & acre & & $\mathrm{lb} / \mathrm{acre}$ & & & $\mathrm{lb}$ \\
\hline $\mathrm{CH}-1 \mathrm{~B}$ & 281.42 & 0.0065 & 50 & 50 & 50 & $10-10-10$ & 3.23 \\
\hline $\begin{array}{c}\mathrm{CH}-1 \mathrm{~B} \\
\text { Topsoil } 1\end{array}$ & 93.8 & 0.0022 & 91.5 & 78.4 & 108.9 & $10-10-10$ & 0.04 \\
\hline $\begin{array}{c}\text { CH-1B } \\
\text { Topsoil } 2\end{array}$ & 93.8 & 0.0022 & 91.5 & 78.4 & 108.9 & $10-10-10$ & 1.92 \\
\hline
\end{tabular}

Seed application began by determining the correct application amount and be seen below. Seed measurement and application methods were the same as in Section 4.3.1.1. Seed mixture application quantities for seed and inoculant included the following:

- Developed Type A

○ Application amount (1.404 lbs.)(636.84 grams) (+/- $0.030 \mathrm{~g})$

○ Inoculant $(4.776 \mathrm{~g})(+/-0.030 \mathrm{~g})$

\section{HECP Application}

On May 28, 2015, Arnold's Custom Seeding, LLC (ACS) arrived on site to begin HECP and soil media application. The HECP used was ProMatrix Engineered Fiber Matrix (EFM) in the same fashion as in Section 4.3.1.1. The soil media used was ProGanics ${ }^{\mathrm{TM}}$ Biotic Soil Media $^{\mathrm{TM}}\left(\mathrm{BSM}^{\mathrm{TM}}\right)$ by Profile Products, LLC at an application rate of $5000 \mathrm{lb} / \mathrm{ac}(5600 \mathrm{~kg} / \mathrm{ha})$ (Profile Products LLC. 2015c). Biotic Earth ${ }^{T M}$ Black Hydraulic Growth Mediums (HGM) was the second growth medium utilized and was hand mixed and applied at an application rate of 6500 $\mathrm{lb} / \mathrm{ac}(7278 \mathrm{~kg} / \mathrm{ha})$. The manufacturer recommended $3500 \mathrm{lb} / \mathrm{ac}(3933 \mathrm{~kg} / \mathrm{ha})$ or greater based on site conditions (Verdyol 2015b). The higher application rate was to account for mixing, handling, and application error. Application of ProMatrix can be seen in Figure 53 and application of ProGanics can be seen in Figure 54 by a FiNN T170 Hydro Seeder owned and operated by ACS. Excess product was mixed in the hydro seeder to ensure proper mixing of the product and application abilities of the machine. 
Biotic Earth application can be seen in Figure 55. The product was donated by Verdyol. The Biotic Earth, $13.8 \mathrm{lb}(6.2 \mathrm{~kg})$, was hand mixed with ProMatrix, $13.8 \mathrm{lb}(6.2 \mathrm{~kg})$, and then applied to the subplots. According to the manufacturer mixing guide, Biotic Earth is best applied by mixing the product with a hydraulic erosion control product (HECP) (Verdyol, N.D.). ProMatrix was the HECP utilized. The HECP was mixed prior in a hydroseeder. The hand mixing of the Biotic Earth was performed in a wheelbarrow by first putting in the HECP and then adding Biotic Earth and water to the mixture. The HECP and HGM mixture was mixed thoroughly with a shovel and then hand applied to the subplots. After application, there were visible bare spots due to difficulty of hand application. The bare spots were then touched up by applying HECP to ensure full coverage of the subplot areas.

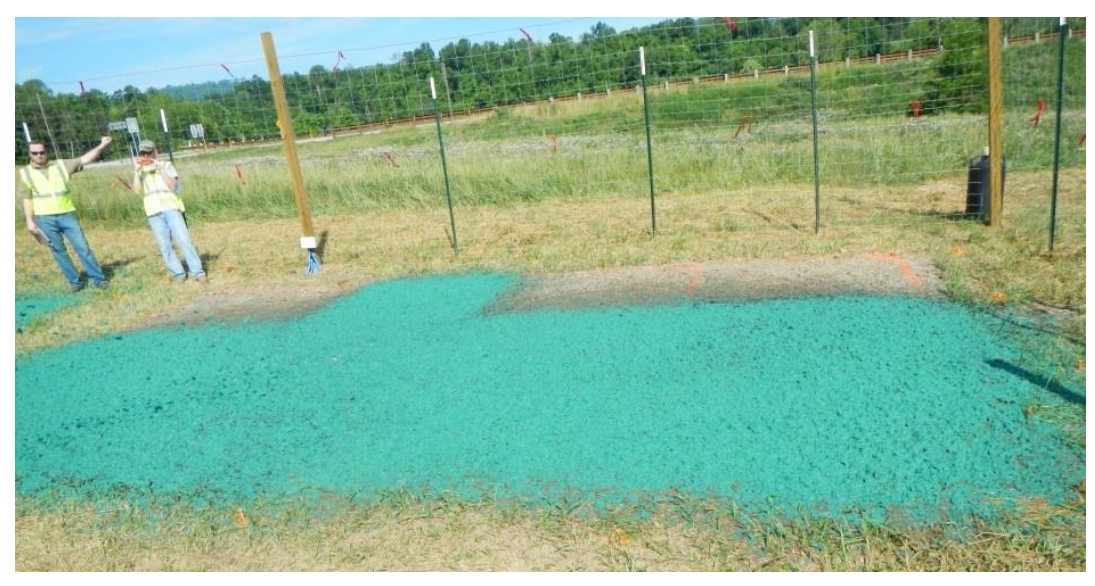

Figure 53: CH-1B HECP Application 


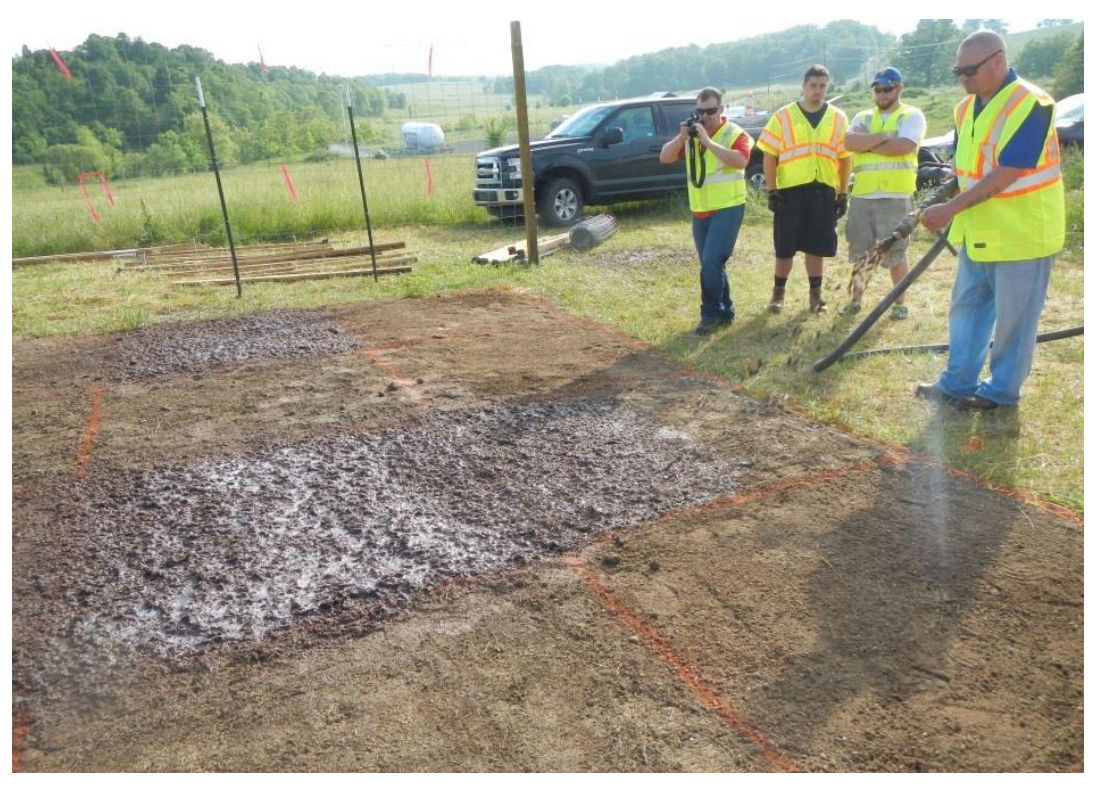

Figure 54: CH-1B ProGanics Application

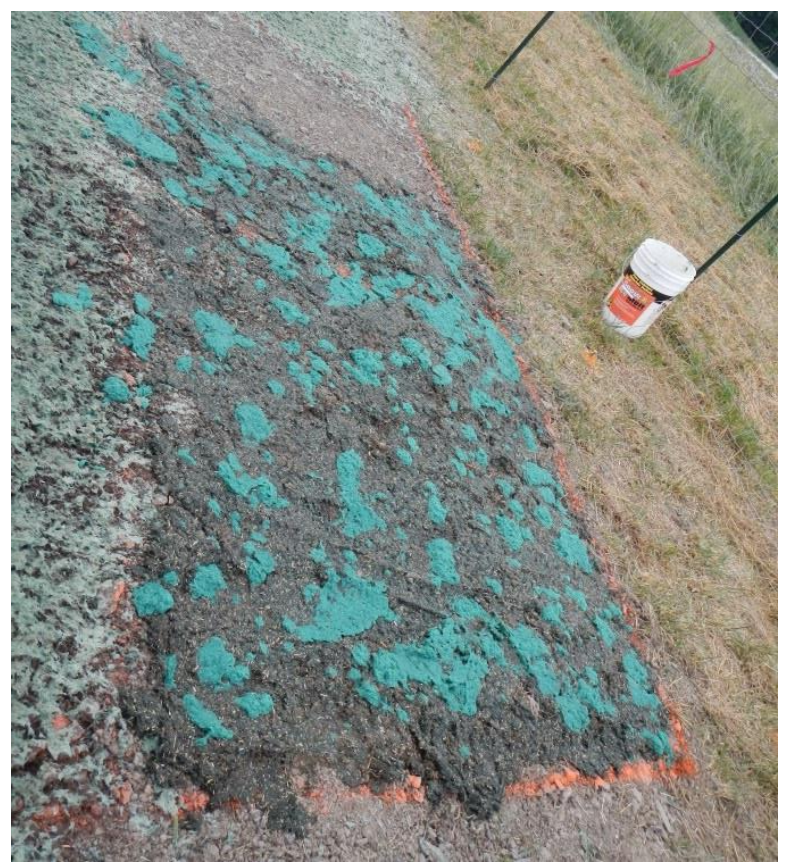

Figure 55: CH-1B Biotic Earth

ProGanics is a biotic soil media developed by Profile Products, LLC and was designed as an alternative to topsoil. The product is composed of thermally refined wood fibers and bark, biochar, biopolymers, seaweed extract, humic acid, and endomycorrhizae. The product also has an organic matter content of $90 \%$, water holding capacity of $800 \%$, pH of 6.0 , and a C:N ratio of 100:1 (Profile Products LLC. 2015d). Once the product is applied, an HECP or rolled 
product is applied for erosion control. ProGanics is not used to control erosion and can be seen in Figure 56.

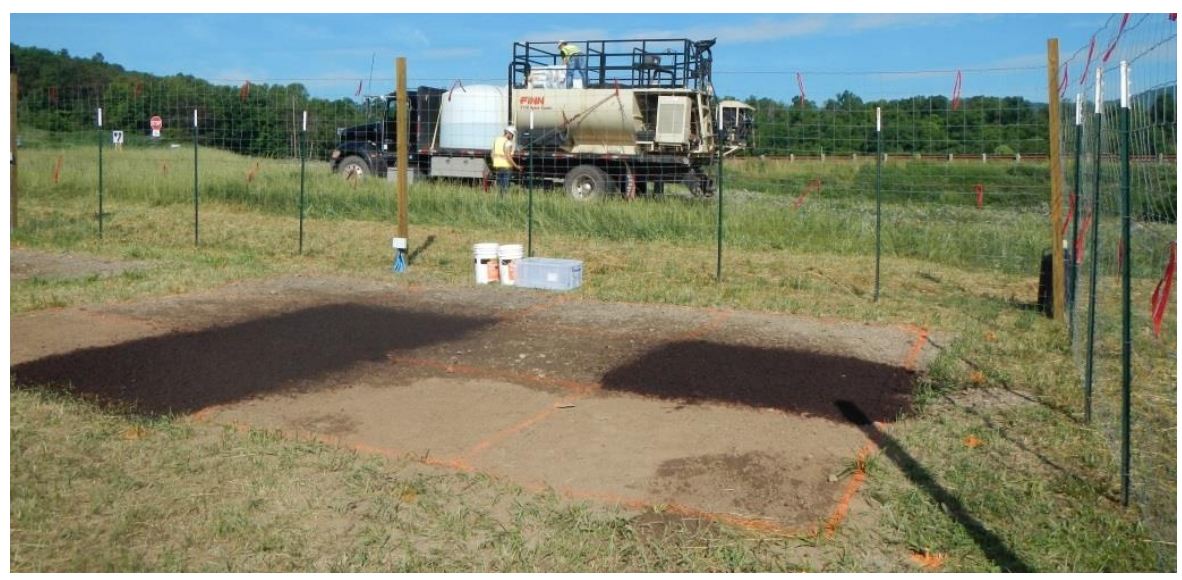

Figure 56: $\mathrm{CH}-1 \mathrm{~B}$ ProGanics

Biotic Earth Black is a hydraulic growth medium that can be used to aid a growth medium for vegetation establishment. The product can also be used for erosion control. The product is produced by Verdyol and is composed of $57 \%$ of thermally and mechanically processed straw and flexible flax fibers. The product also contains $40 \%$ sphagnum peat moss and less than $1 \%$ of Mycorise Pro. There is also $3 \%$ alfalfa meal, sugars, starches, proteins, fiber, 16 amino acids, vitamin A, and tricontanal growth stimulant. The prodcut is reported to have gretater than $95 \%$ organtic matter content, C:N ratio of $31: 1$, moisture content of $44.5 \%$, and a pH of 5.5 (Verdyol 2015a).

\section{Weed Removal}

As previously discussed in Section 4.3.1.1., Jimson weed (Datura stramonium) was removed to address overcrowding concerns on June 24, 2015. The plot area before plant removal can be seen in Figure 57 and after species removal can be seen in Figure 58. No other species removal occurred through the remainder of the study. 


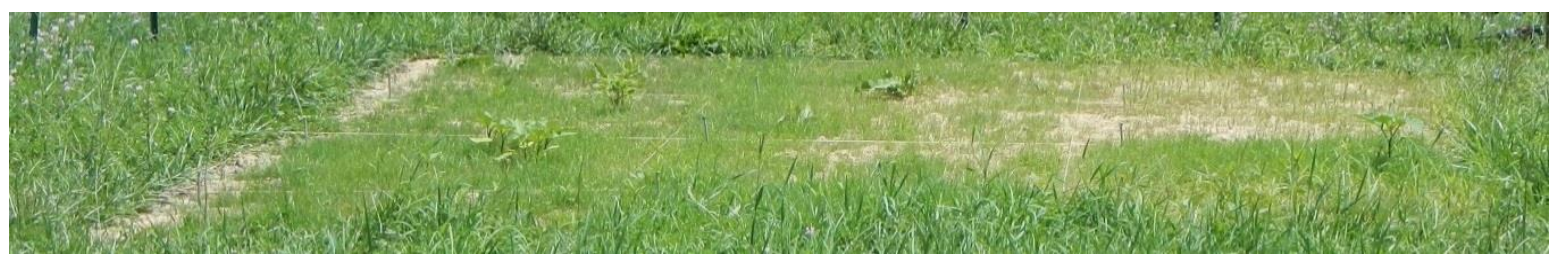

Figure 57: CH-1B Before Removal

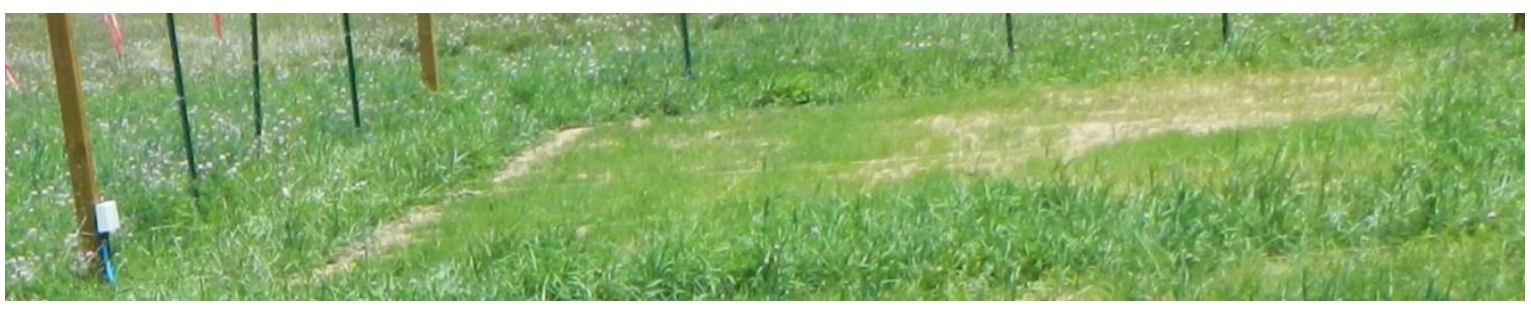

Figure 58: CH-1B After Removal

\subsubsection{Data Collection}

\subsubsection{Topography}

The topography was recorded using an FC-200 Data Collector and Hiper Lite Plus from Topcon. Points at the corners and center and any notable changes in topography were recorded by the Topcon system for each plot. The information was used to determine the slope and geographic location information. A SUUNTO A-10 recreational compass was used to determine the heading of each subplot. The compass, manufactured by SUUNTO, has an accuracy of $2.5^{\circ}$ and compass resolution of $2^{\circ}$. A GARMIN eTrex 20 handheld GPS was also used to record the GPS and elevation data. The GPS is manufactured by GARMIN, is operational in a temperature range of -20 to $70 \mathrm{C}^{\circ}$, and has accuracy within $+/-49 \mathrm{ft}(+/-15 \mathrm{~m})$ horizontal and +/- 1,312 (+/$400 \mathrm{~m}$ ) vertical. A SUUNTO PM-5 clinometer was used to determine the slope for the plot and subplots. The clinometer, manufactured by SUUNTO, has an inclination accuracy of $1 / 4^{\circ}$ and inclination resolution of $1^{\circ}$ and $1 \%$. All topographic measurements were taken before the planting stage indicated in Appendix 7.1. 


\subsubsection{Vegetation}

Vegetation was examined by using a $3.3 \mathrm{ft} \times 3.3 \mathrm{ft}(1 \mathrm{~m} \times 1 \mathrm{~m})$ Vegetation Measuring Device (VMD) with a grid containing 100 intersections (Calloudon et al. 1996 and Elzinga et al. 1998). The VMD measured the percent cover in each subplot. A picture of each VMD examination was taken with a Nikon COOLPIX AW100 camera with built in GPS. The pictures were taken back to the lab to determine the percent cover by species using Adobe Photoshop CC software. For each VMD measurement, the prominent species and site characteristics were recorded. Vegetation measurements were conducted approximately every two weeks after the planting stage (see Appendix 7.1).

\subsubsection{Precipitation}

At each location, there was one WatchDog Tipping Bucket Rain Gauge manufactured by Spectrum Technologies, Inc to measure the site precipitation. The device was installed during the planting stage. The device was mounted on a wood post, kept at minimum $1 \mathrm{ft}(0.3 \mathrm{~m})$ from the woven fence, and checked for level once a month. The device was placed in an open area to avoid vegetation interfering with the equipment. The rain gauge has an accuracy of $+/-2 \%$ if rainfall is less than $0.16 \mathrm{ft} / \mathrm{hr}(5 \mathrm{~cm} / \mathrm{hr})$. The tipping bucket rain gauge must be cleaned once or twice a year with a recommended battery replacement once a year. The data from the rain gauge was connected to Channel A and recorded on a 60 minute interval by a WatchDog 1425 Micro Station and collected according to the examine dates in Appendix 7.1. The data were downloaded every two weeks on field visits.

\subsubsection{Temperature}

Ambient air temperature at all sites was measured every 60 minutes with a WatchDog 1425 Micro Station produced by Spectrum Technologies, Inc. The 1425 model has an internal temperature reader that has a temperature range of -40 to $185^{\circ} \mathrm{F}\left(-40\right.$ to $\left.85^{\circ} \mathrm{C}\right)$. The accuracy of the device is $\pm 1.1^{\circ} \mathrm{F}$ at -4 to $122^{\circ} \mathrm{F}\left( \pm 0.6^{\circ} \mathrm{C}\right.$ at -20 to $\left.50^{\circ} \mathrm{C}\right)$, otherwise $\pm 2.2^{\circ} \mathrm{F}\left( \pm 1.2^{\circ} \mathrm{C}\right)$. 
A SMEC 300 soil moisture sensor was also used to measure soil temperature every 60 minutes. The sensor had a thermistor potted in the sensor molding and the sensor was installed $0.5 \mathrm{ft}(0.15 \mathrm{~m})$ below the soil surface. The accuracy of the sensor is $\pm 1^{\circ} \mathrm{F}\left( \pm 0.6^{\circ} \mathrm{C}\right), \mathrm{T}>-25^{\circ} \mathrm{F}(-$ $\left.30^{\circ} \mathrm{C}\right)$ and $\pm 1.5^{\circ} \mathrm{F}\left( \pm 0.6^{\circ} \mathrm{C}\right), \mathrm{T}<-25^{\circ} \mathrm{F}\left(-30^{\circ} \mathrm{C}\right)$. The sensor has a range of -60 to $185^{\circ} \mathrm{F}(-50$ to $\left.85^{\circ} \mathrm{C}\right)$. The resolution of the sensor is $0.1^{\circ} \mathrm{F}\left(0.1^{\circ} \mathrm{C}\right)$. The sensor had dimensions of 1.8 in $\times 1.2$ in $(7 \mathrm{~cm} \times 3 \mathrm{~cm})$ and an oscillator frequency of $80 \mathrm{MHz}$. The device cable was $20 \mathrm{ft}(6 \mathrm{~m})$ and made by Spectrum Technologies, Inc. Channel D in a WatchDog 1425 or 1400 Micro Station was used to record the data from the sensor. The data were downloaded every two weeks on field visits.

\subsubsection{Soil}

A soil auger was used to collect soil samples from each subplot to create a composite for testing. The sampling was a composite of the plot before planting, so that appropriate lime and fertilizer recommendations could be made. Erosion factors were visually inspected and recorded during each visit. The composite soil sample was then sent to AgSource Laboratories in Lincoln, Nebraska to test for $\mathrm{pH}$, soluble nutrients, and particle size. An agraTronix Soil Compaction Tester manufactured by agraTronix was used to test the compaction of the subplots. The penetrometer complies to and is based on the ASAE S313.3 standard. The method followed complies with the ASAE EP542 code. Compaction was tested once at the planting stage and once at the end of the study for each subplot.

A SMEC 300 soil moisture sensor was used to measure volumetric water content (VWC) and electrical conductivity $(E C)$ every 60 minutes at each site location. The sensor was installed $0.5 \mathrm{ft}(0.15 \mathrm{~m})$ below the soil surface. The VWC accuracy is $3 \%$ and EC accuracy $\pm 2 \%$. The sensor has a range of VWC $0 \%$ to saturation, EC 0 to $10 \mathrm{mS} / \mathrm{cm}$. The resolution of the sensor is VWC $0.1 \%$ and EC $0.01 \mathrm{mS} / \mathrm{cm}$. The sensor had dimensions of $1.8 \mathrm{in} \times 1.2$ in $(7 \mathrm{~cm} \times 3 \mathrm{~cm})$ and an oscillator frequency of $80 \mathrm{MHz}$. The device cable was $20 \mathrm{ft}(6 \mathrm{~m})$ and made by Spectrum 
Technologies, Inc. Channel D in a WatchDog 1425 or 1400 Micro Station was used to record the data from the sensor.

A SM 100 soil moisture sensor was used to measure volumetric water content (VWC) every 60 minutes for objective 3 and 4 and downloaded every two weeks. The sensor was installed $0.5 \mathrm{ft}(0.15 \mathrm{~m})$ below the soil surface. The VWC accuracy is $3 \% \mathrm{VWC}$ at $\mathrm{EC}<8 \mathrm{mS} / \mathrm{cm}$. The sensor has a range of VWC $0 \%$ to saturation and a resolution of $0.1 \%$. The sensor had dimensions of 2.4 in $\times 0.8$ in $\times 0.1$ in $(6 \mathrm{~cm} \times 2 \mathrm{~cm} \times 0.3 \mathrm{~cm})$ and an oscillator frequency of 80 $\mathrm{MHz}$. The device cable was $20 \mathrm{ft}(6 \mathrm{~m})$ and made by Spectrum Technologies, Inc. Channel $\mathrm{D}$ in a WatchDog 1425 or 1400 Micro Station was used to record the data from the sensor.

\subsubsection{Micro Stations}

WatchDog 1425 and 1400 Micro Stations were used to collect and record data. The sensors used in the field were a SM 100 and SMEC soil moisture sensors and tipping bucket rain gauge. The data loggers have an operating temperature range of $-22^{\circ}$ to $130^{\circ} \mathrm{F}\left(-30^{\circ}\right.$ to $\left.55^{\circ} \mathrm{C}\right)$. The micro stations are manufactured by Spectrum Technologies, Inc. SpecWare 9 Professional software was purchased in order to download data from the micro stations. The software is produced by Spectrum Technologies, Inc. The software also allows for easy view, storage, and management of the collected data.

\subsubsection{Data Analysis}

\subsubsection{Percent Cover}

A VMD was used to examine percent cover for each objective. The data were then compiled to develop percent cover bar graphs over the course of the study period. The photos of the VMD at 51 and 90 days were examined for cover based on species.

\subsubsection{Statistical Analysis}

The experiments were all setup as complete randomized designs (Lyman and Longnecker 2001). A one-way ANOVA and paired student's t test were then used to analyze the 
percent cover data at 8 days and 90 days after planting for Objective 1, 2, and 4. A one-way blocked ANOVA and paired student's t test were used to analyze Objective 3 percent cover data. The one-way blocked ANOVA examined if there were any statistical differences between the various seed mixtures and treatments. JMP Pro 12 was used to run all statistical analyses.

\subsubsection{Monitoring Data}

Field sensors were used to collect data on precipitation, ambient air and soil temperature, volumetric water content, and electrical conductivity. The data were collected and processed with SpecWare 9 Professional. After processed, the data was loaded into Microsoft Excel to develop graphs to display the information in a user friendly format for comparison and discussion purposes.

\subsection{Results}

\subsubsection{Objective 1: Comparison with current standard}

\subsubsection{Vegetation Measurements}

The percent cover of six mixtures was examined over a 90 day period starting on May 27, 2015. DOH Medians, Mowable Areas, Cool Season, and High Elevation mixtures all achieved $70 \%$ coverage after 24 days from planting. DOH Cut/Fill and Warm Season seed mixtures at $68 \%$ and $51 \%$ coverage were unable to achieve $70 \%$ coverage in the same timeframe. From 35 to 90 days, all seed mixtures exceeded the $70 \%$ coverage bench mark. The percent cover results can be seen in Figure 59 and the data can be seen in Appendix 7.2. 


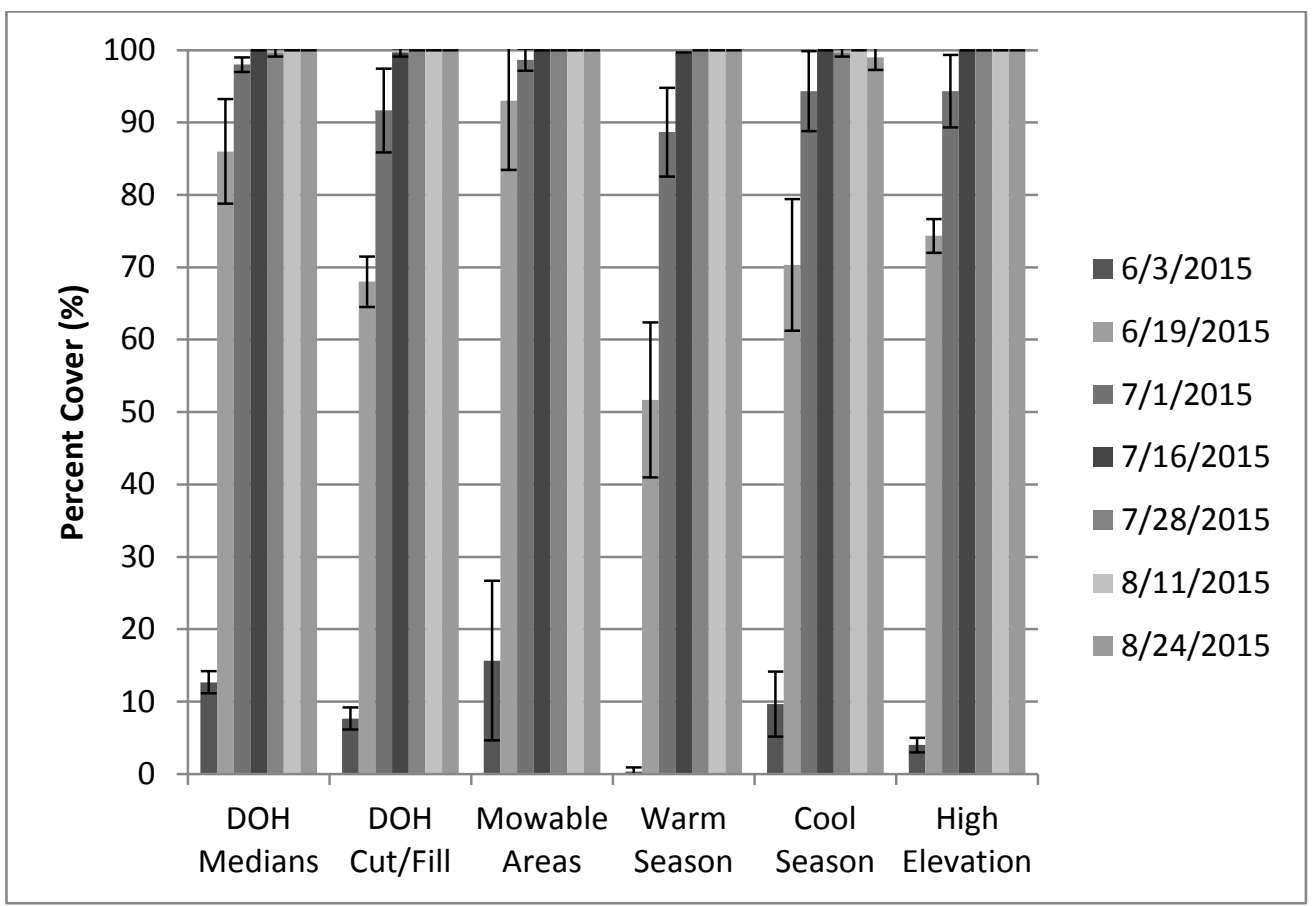

Figure 59: Objective 1 Percent Cover

Percent cover by species was examined 51 days after planting. Mowable Areas mixture had the highest percent of not planted species at 35\% coverage. High Elevation mixture had the highest percent of planted species at $96 \%$ coverage. DOH Medians and DOH Cut/Fill had a planted coverage of $83 \%$ and $82 \%$. The results can be seen in Figure 60 and the data can be seen in Appendix 7.2. 


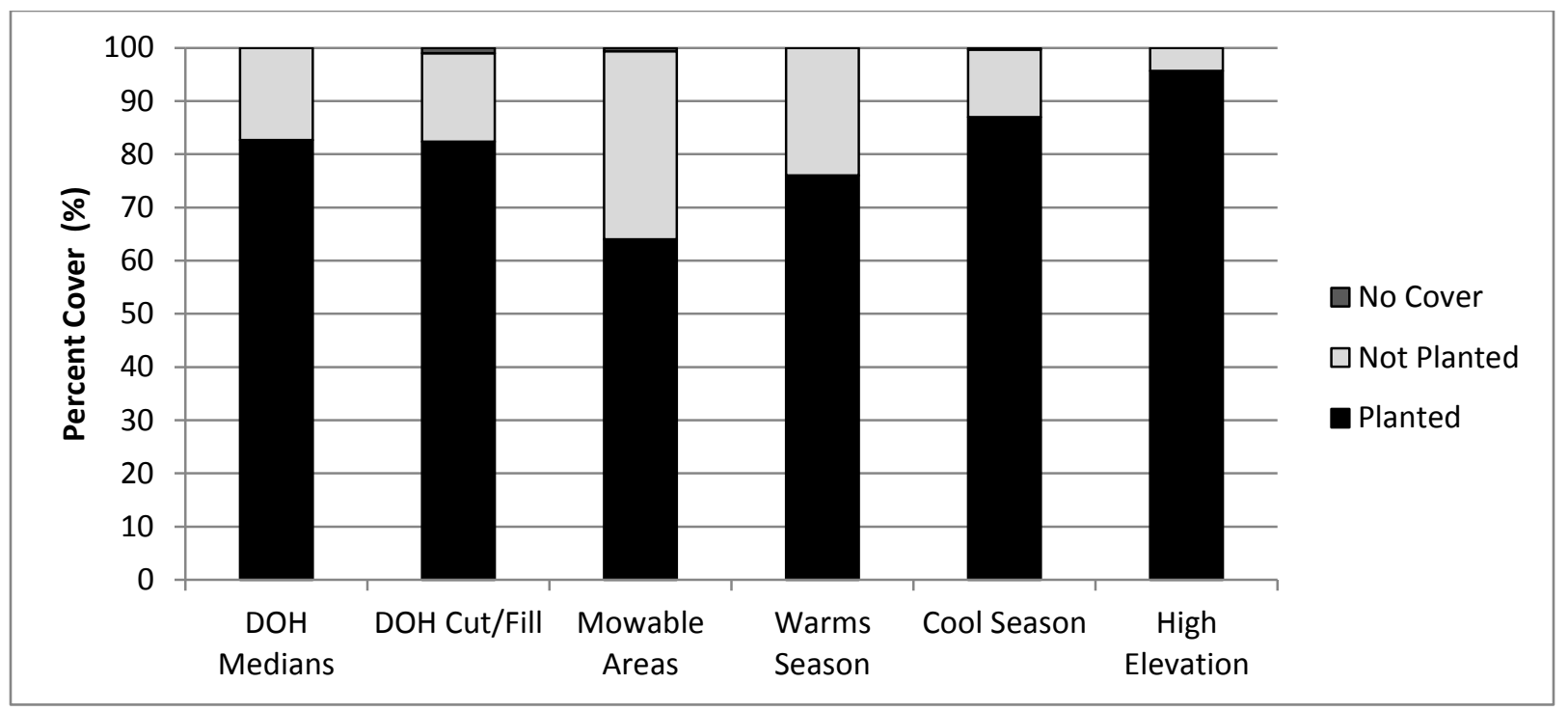

Figure 60: Objective 1 Percent Cover by Species at 51 Days

Percent cover by species was also examined 90 days after planting. Mowable Areas mixture had the highest percent of not planted species at $38 \%$ coverage and the lowest percent planted at $62 \%$. High Elevation mixture had the highest percent of planted species at $88 \%$ coverage. The results can be seen in Figure 61 and the data can be seen in Appendix 7.2.

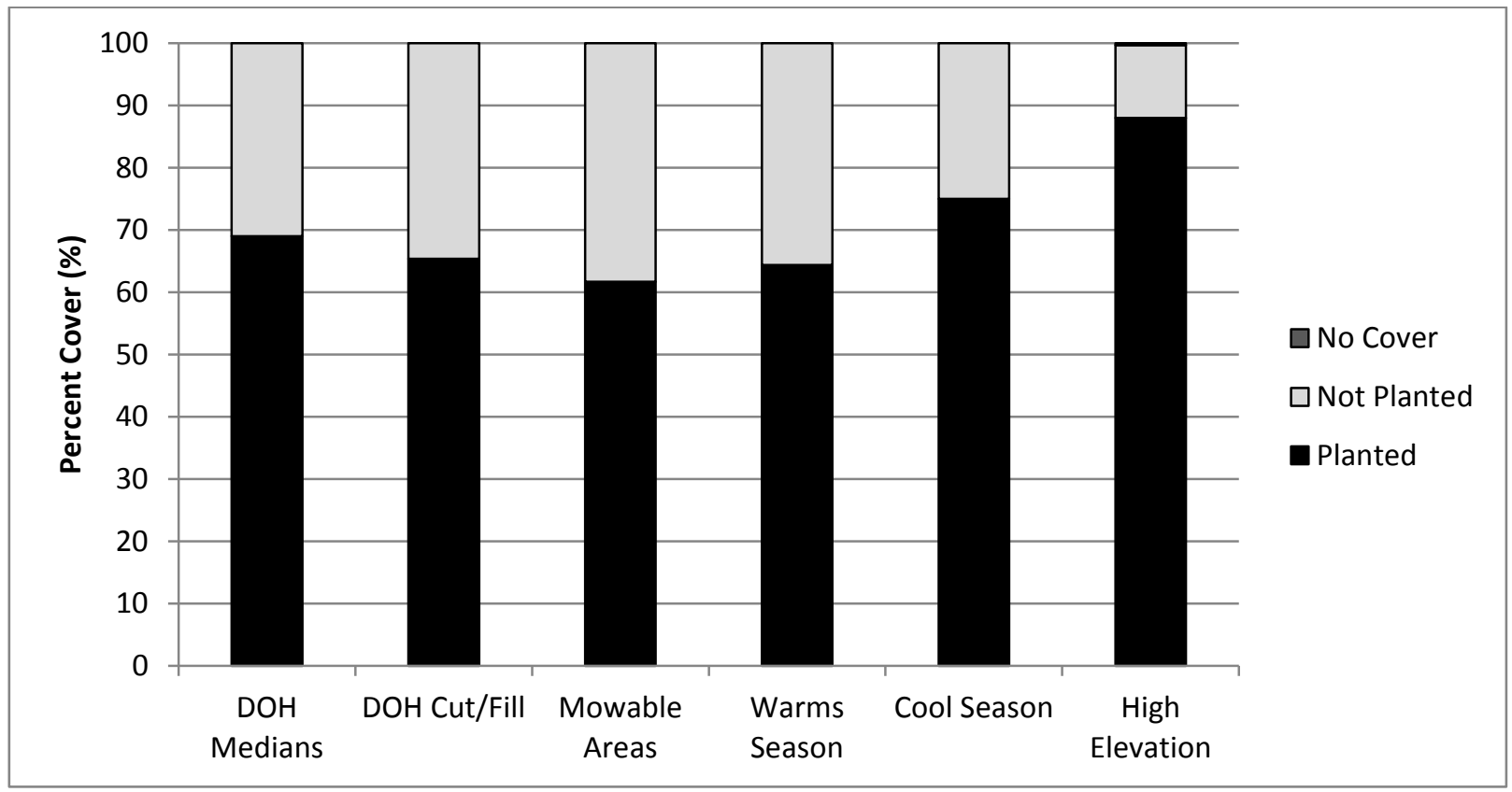

Figure 61: Objective 1 Percent Cover by Species at 90 Days 
After eight days from planting, a one-way ANOVA analysis indicated there was a significant difference between the various seed mixtures seen in Figure 62. A p-value of 0.0261 was determined and can been seen in Table 23. The two native warm season mixtures, High Elevation and Warm Season, indicated a slower initial germination compared to the non-native cool season mixtures (Cool Season, DOH Cut/Fill, DOH Medians, and Mowable Areas). Warm season species are commonly noted to be slow to germinate and establish compared to cool season species (Salon and Miller 2012) and this relationship can be seen in Figure 62.

Table 23: Objective 1 Eight Days One-way ANOVA Statistics

\begin{tabular}{lccccc}
\hline Source & DF & $\begin{array}{c}\text { Sum of } \\
\text { Squares }\end{array}$ & $\begin{array}{c}\text { Mean } \\
\text { Square }\end{array}$ & F Ratio & Prob > F \\
\hline Treatment & 5 & 472.66667 & 94.5333 & 3.8411 & $0.0261^{*}$ \\
Error & 12 & 295.33333 & 24.6111 & & \\
C. Total & 17 & 768.00000 & & & \\
\hline
\end{tabular}

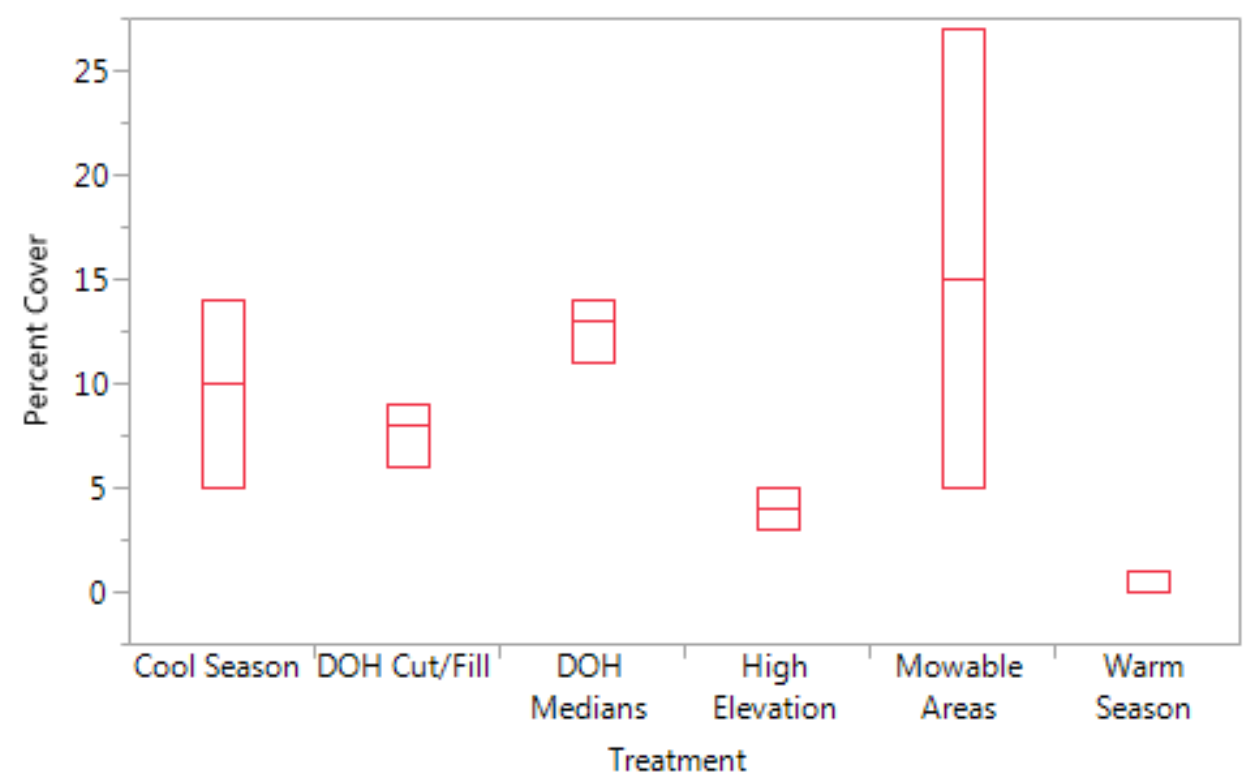

Figure 62: Objective 1 Eight Days One-way ANOVA Graph; $n=3 ; 25 \%-75 \%$ (box); median (50\% line in box)

A paired student's t test was also performed on the percent cover data after eight days from planting. A table of $p$-values and a connecting letters report from the student's test can be seen in Table 24 and 25. The test indicated that Mowable Areas, DOH Medians, and Cool Season mixtures performed statistically better compared to the Warm Season mixture based on 
mean percent cover. The test also indicated that Mowable Areas had a higher mean value of percent cover compared to the examined Warm Season mixture with a p-value of 0.0138 .

Table 24: Objective 1 Eight Days Paired Student's t Test $p$-Values

\begin{tabular}{llr}
\hline Level & - Level & p-Value \\
\hline Mowable Areas & Warm Season & $0.0026^{*}$ \\
DOH Medians & Warm Season & $0.0102^{*}$ \\
Mowable Areas & High Elevation & $0.0138^{*}$ \\
Cool Season & Warm Season & $0.0399^{*}$ \\
DOH Medians & High Elevation & 0.0536 \\
Mowable Areas & DOH Cut/Fill & 0.0717 \\
DOH Cut/Fill & Warm Season & 0.0953 \\
Mowable Areas & Cool Season & 0.1643 \\
Cool Season & High Elevation & 0.1871 \\
DOH Medians & DOH Cut/Fill & 0.2407 \\
DOH Cut/Fill & High Elevation & 0.3832 \\
High Elevation & Warm Season & 0.3832 \\
Mowable Areas & DOH Medians & 0.4732 \\
DOH Medians & Cool Season & 0.4732 \\
Cool Season & DOH Cut/Fill & 0.6304 \\
\hline
\end{tabular}

Table 25: Objective 1 Eight Days Connecting Letters Report

\begin{tabular}{lrrr}
\hline Level & & & Mean \\
\hline Mowable Areas & A & & 15 \\
DOH Medians & A B & 12 \\
Cool Season & A B & 9 \\
DOH Cut/Fill & A B C & 7 \\
High Elevation & B C & 4 \\
Warm Season & & C & 0.3 \\
\hline
\end{tabular}

After ninety days from planting, a one-way ANOVA analysis indicated there was no significant difference between the various seed mixtures seen in Figure 63. A p-value of 0.95 was determined and can been seen in Table 26. The two native mixtures, High Elevation and Warm Season, did not show any significant difference over long term growth during establishment compared to the non-native mixtures, Cool Season, DOH Cut/Fill, DOH Medians, and Mowable Areas. 
Table 26: Objective 1 Ninety Days One-way ANOVA Statistics

\begin{tabular}{lccccc}
\hline Source & DF & $\begin{array}{c}\text { Sum of } \\
\text { Squares }\end{array}$ & $\begin{array}{c}\text { Mean } \\
\text { Square }\end{array}$ & F Ratio & Prob $>$ F \\
\hline Treatment & 5 & 1186.44 & 237.29 & 0.2214 & 0.9527 \\
Error & 120 & 128586.10 & 1071.55 & & \\
C. Total & 125 & 129772.54 & & & \\
\hline
\end{tabular}

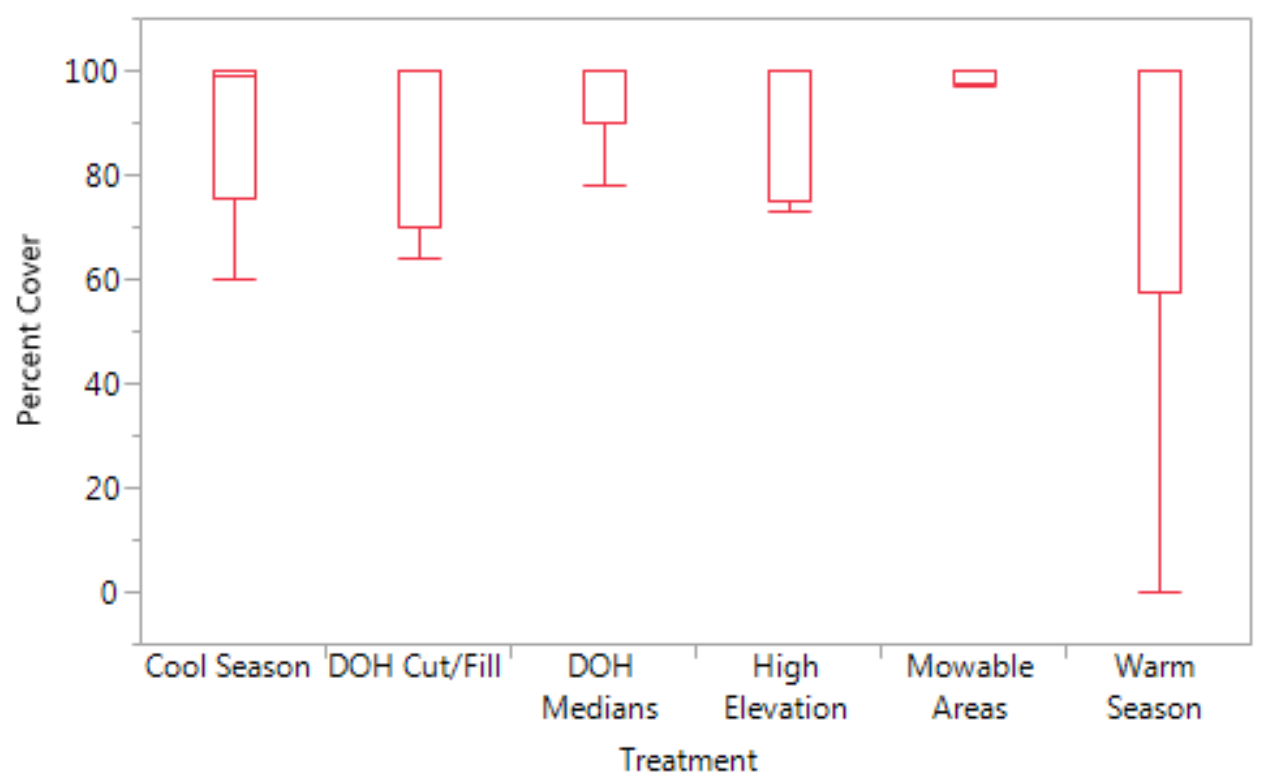

Figure 63: Objective 1 Ninety Days One-way ANOVA Graph; min and max (whisker); 25\%-75\% (box); median $(50 \%$ line in box $)$

\subsubsection{Monitoring Data}

The precipitation and mean ambient air temperature were monitored over the course of the study and can be viewed in Figure 64. The months of June, July, and August saw a precipitation accumulation of $6.15,2.17$, and 2.28 in $(15.62,5.51$, and $5.79 \mathrm{~cm})$. A total accumulation of 11.28 in $(28.65 \mathrm{~cm})$ were documented from May 27 to August 24, 2015. The largest quantity of precipitation in one day was on June 19, 2015 totally to 1.9 in $(4.8 \mathrm{~cm})$. The highest monthly mean ambient air temperature was for the month of July at $76^{\circ} \mathrm{F}\left(24^{\circ} \mathrm{C}\right)$ which also the highest mean daily temperature at $84^{\circ} \mathrm{F}\left(29^{\circ} \mathrm{C}\right)$. The lowest mean temperature occurred May 6, 2015 at $59.2^{\circ} \mathrm{F}\left(15.1^{\circ} \mathrm{C}\right)$. 


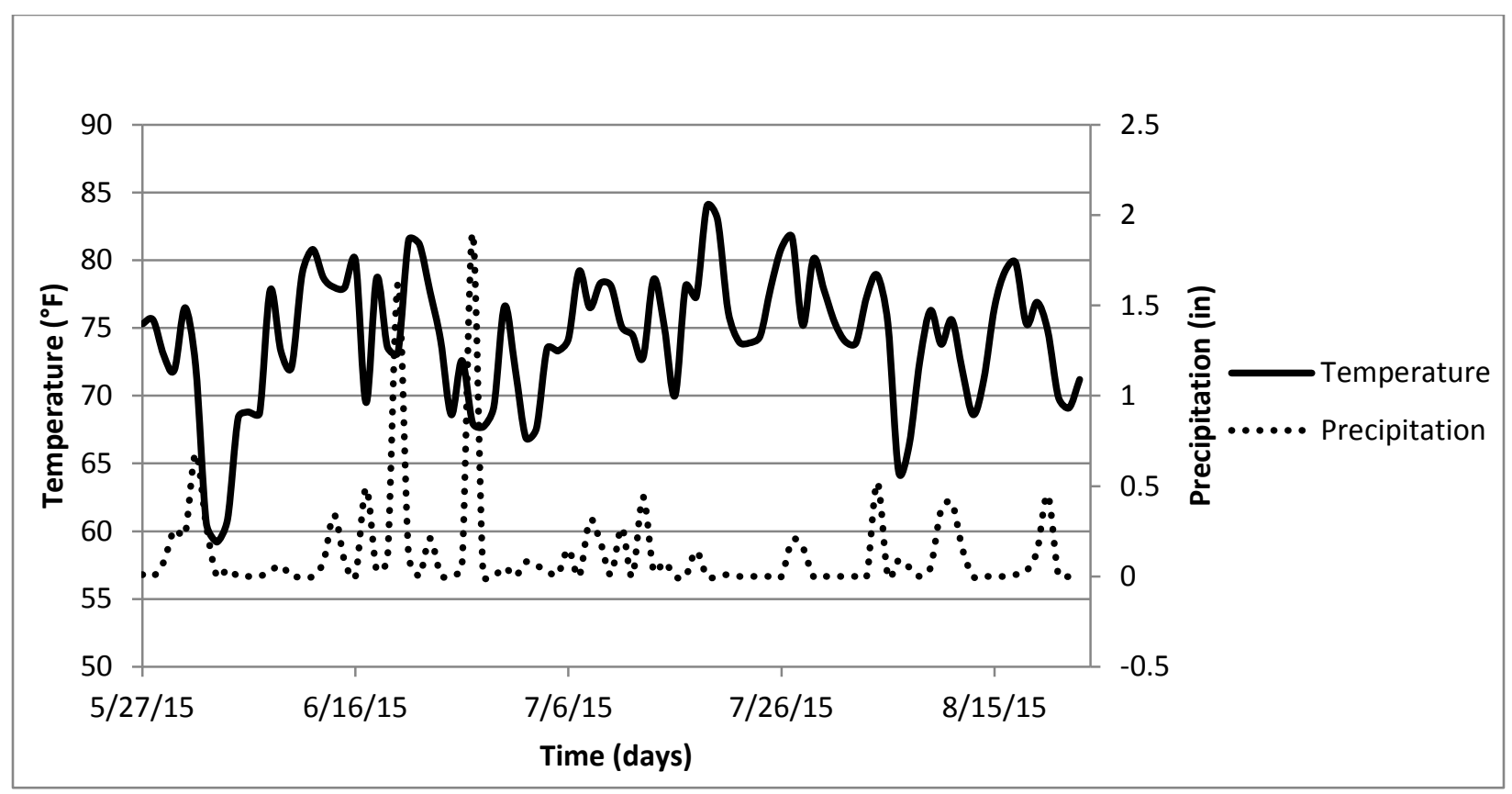

Figure 64: Objective 1 Weather Conditions

The mean soil temperature and volumetric water content was monitored over the course of the study and can be viewed in Figure 65. The mean soil temperatures for the month of June, July, and August were $74.4^{\circ} \mathrm{F}, 74.9^{\circ} \mathrm{F}$, and $72.8^{\circ} \mathrm{F}\left(23.6^{\circ} \mathrm{C}, 23.8^{\circ} \mathrm{C}\right.$, and $\left.22.7^{\circ} \mathrm{C}\right)$. The highest daily soil temperature was recorded on the first day of planting at $80.2^{\circ} \mathrm{F}\left(26.8^{\circ} \mathrm{C}\right)$ due to the fact black plastic was covering the site to prevent unwanted vegetation establishment before planting. The black plastic was absorbing heat and transferring it to the bare soil surface. The second highest soil temperature was documented on June 22,2015 at $79.3^{\circ} \mathrm{F}\left(26.3^{\circ} \mathrm{C}\right)$ under normal vegetation conditions.

Volumetric water content stayed between 32\%-41\% from the end of May to the start of July. The volumetric water content started to decrease at the beginning of July resulting in a $6.6 \%-14.3 \%$ range from late July to the end of the study. The high and low percent in volumetric water content closely resembled the spring and summer seasons in West Virginia. The transition in percent volumetric water content in July fell close to the official change in seasons. West Virginia commonly experiences moist conditions in spring and dryer conditions in the summer which can be approximately seen in Figure 64. 


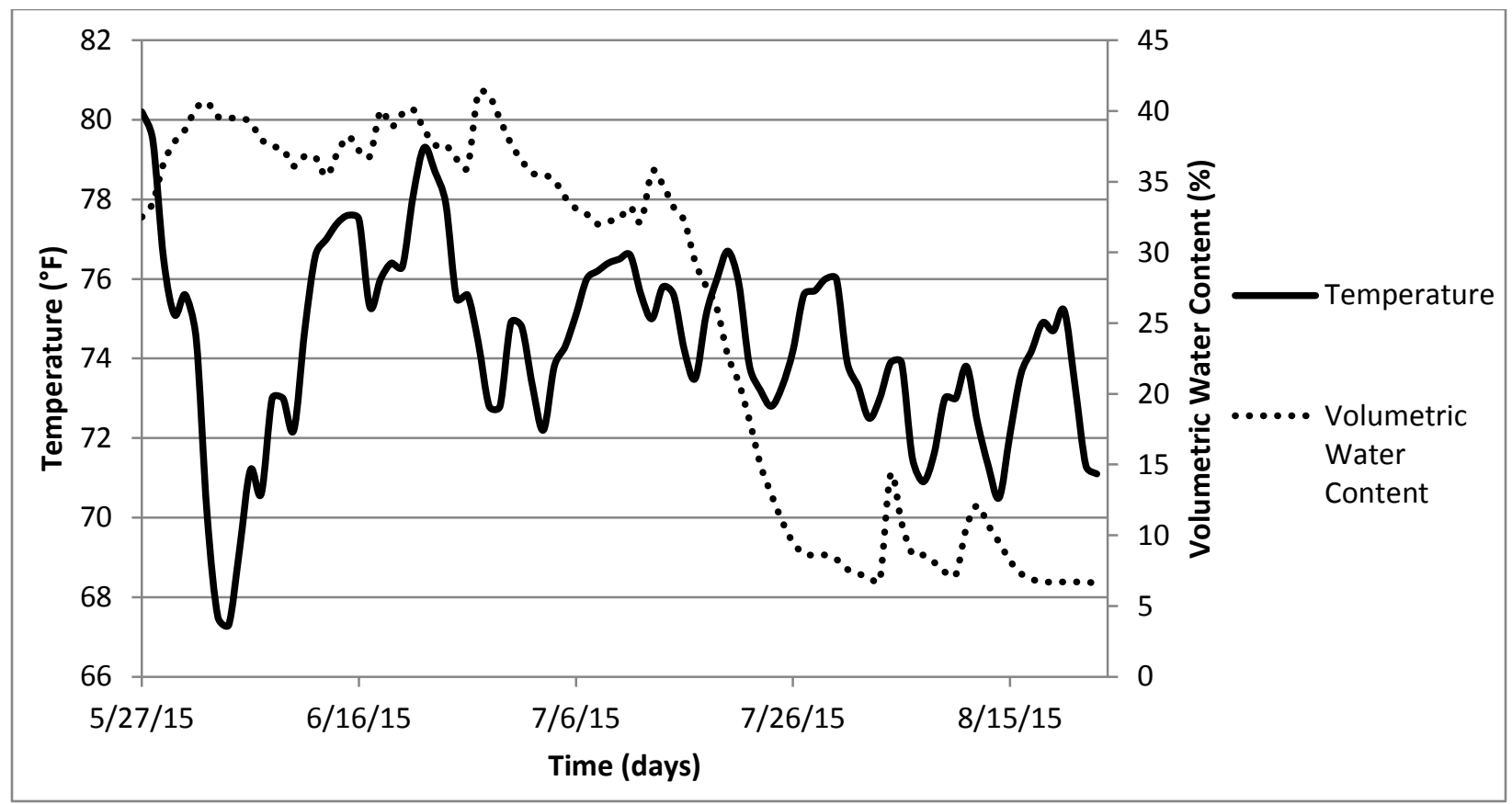

Figure 65: Objective 1 Soil Characteristics

Electrical conductivity of the soil was monitored with a soil sensor and can be seen graphed in Figure 66.

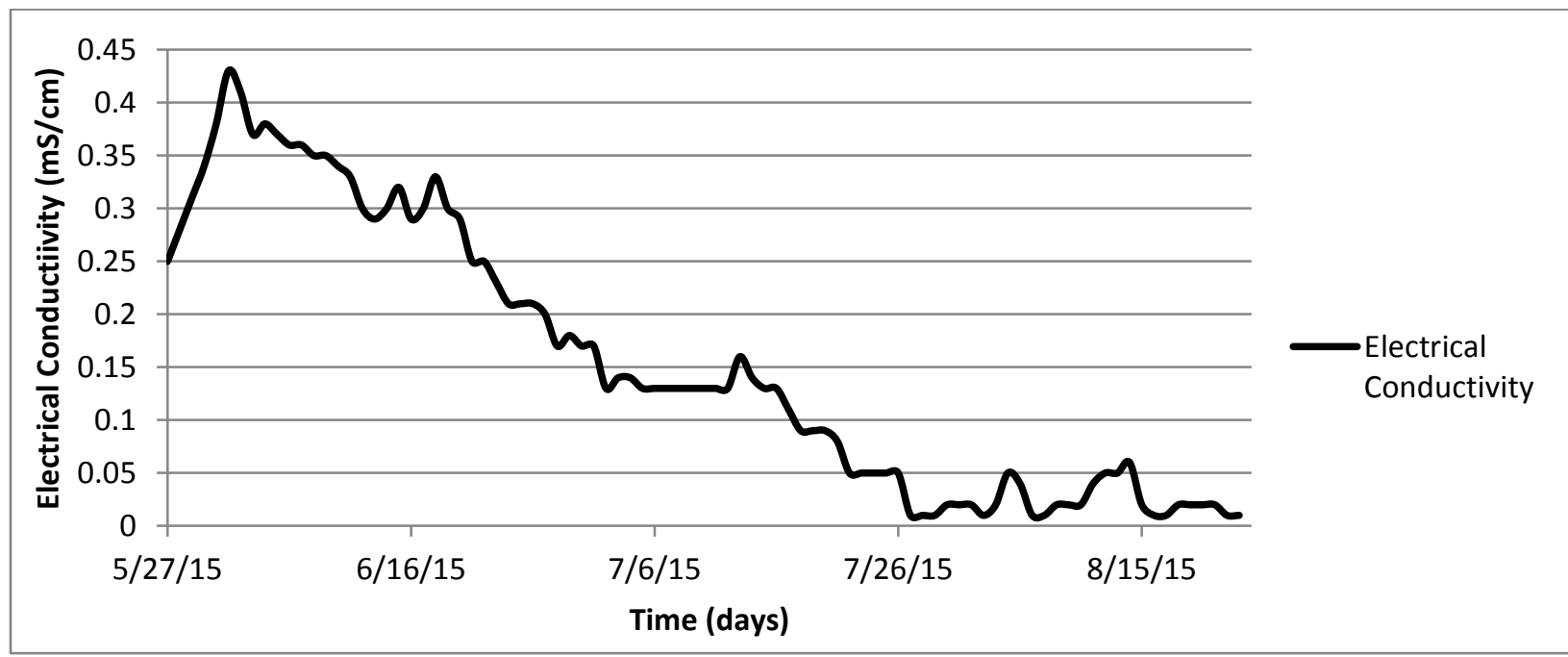

Figure 66: Objective 1 Mean Electrical Conductivity

Compaction data was taken before and after seedbed preparations. The mean compaction data for the overall site and subplots can be seen in Table 27 . Table 28 illustrates the significance of the reported values. A rank of "Green" means the soil displays optimum compaction conditions for plant root development, 0-200 psi approximately. A "Yellow" rank 
indicates soil conditions that have moderate conditions for plant development, 200-300 psi approximately. A "Red" rank demonstrates poor compaction conditions with an approximate compaction of $\geq 300$ psi. A rank of "Max" indicates the penetrometer exceeded reading capabilities and the soil layer was over compacted. The top 9 in. of soil are significantly important because compaction at that depth can be altered through mechanical tillage to improve site conditions for root and plant development.

Looking at the compaction data in Table 27, the initial compaction of the site only provided poor compaction conditions in the first 3 in. From 6-9 in., the soil layers did not provide good growing conditions for root development. After site preparation, the first 3 in. in general raised to a rank of "Yellow" improving the compaction conditions. The next 6 in. improved as well, but did not reach a compaction level significant for good root development.

Table 27: CH-1A Compaction Data

\begin{tabular}{|c|c|c|c|c|c|c|c|}
\hline \multirow{2}{*}{$\begin{array}{l}\text { Penetrometer } \\
\text { Depth } \\
\text { Markings } \\
\text { (in.) }\end{array}$} & $\begin{array}{l}\text { Before Site } \\
\text { Preparation }\end{array}$ & \multicolumn{6}{|c|}{ After Site Preparation } \\
\hline & $\mathrm{CH}-1 \mathrm{~A}$ & $\begin{array}{c}\mathrm{DOH} \\
\text { Medians }\end{array}$ & $\begin{array}{c}\mathrm{DOH} \\
\text { Cut/Fills }\end{array}$ & $\begin{array}{c}\text { Mowable } \\
\text { Areas }\end{array}$ & $\begin{array}{l}\text { Warm } \\
\text { Season }\end{array}$ & $\begin{array}{c}\text { Cool } \\
\text { Season }\end{array}$ & $\begin{array}{c}\text { High } \\
\text { Elevation }\end{array}$ \\
\hline 3" Color: & 1 & 1.7 & 1.8 & 1.0 & 1.3 & 1.0 & 1.3 \\
\hline 6" Color: & 0.5 & 0.8 & 1.3 & 0.8 & 0.8 & 0.5 & 0.5 \\
\hline 9" Color: & 0 & 0.3 & 0.3 & 0.3 & 0.3 & 0.0 & 0.0 \\
\hline
\end{tabular}

Table 28: Compaction Data Classification

\begin{tabular}{lc}
\hline Rank & Scale \\
\hline Green & $2.1-3.0$ \\
Yellow & $1.1-2.0$ \\
Red & $0.6-1.0$ \\
Max & $0-0.5$ \\
\hline
\end{tabular}

\subsubsection{Objective 2: Evaluating high elevation needs}

\subsubsection{Vegetation Measurement}

The percent covers of five mixtures were examined over a 90 day period. DOH Medians, Mowable Areas, and Cool Season mixtures all achieved $70 \%$ coverage over the duration of the 
study. Warm Season and High Elevation seed mixtures only reached a maximum coverage of $62.7 \%$ and $58.3 \%$ by the end of the study. The percent cover results can be seen in Figure 67 and the data can be seen in Appendix 7.3.

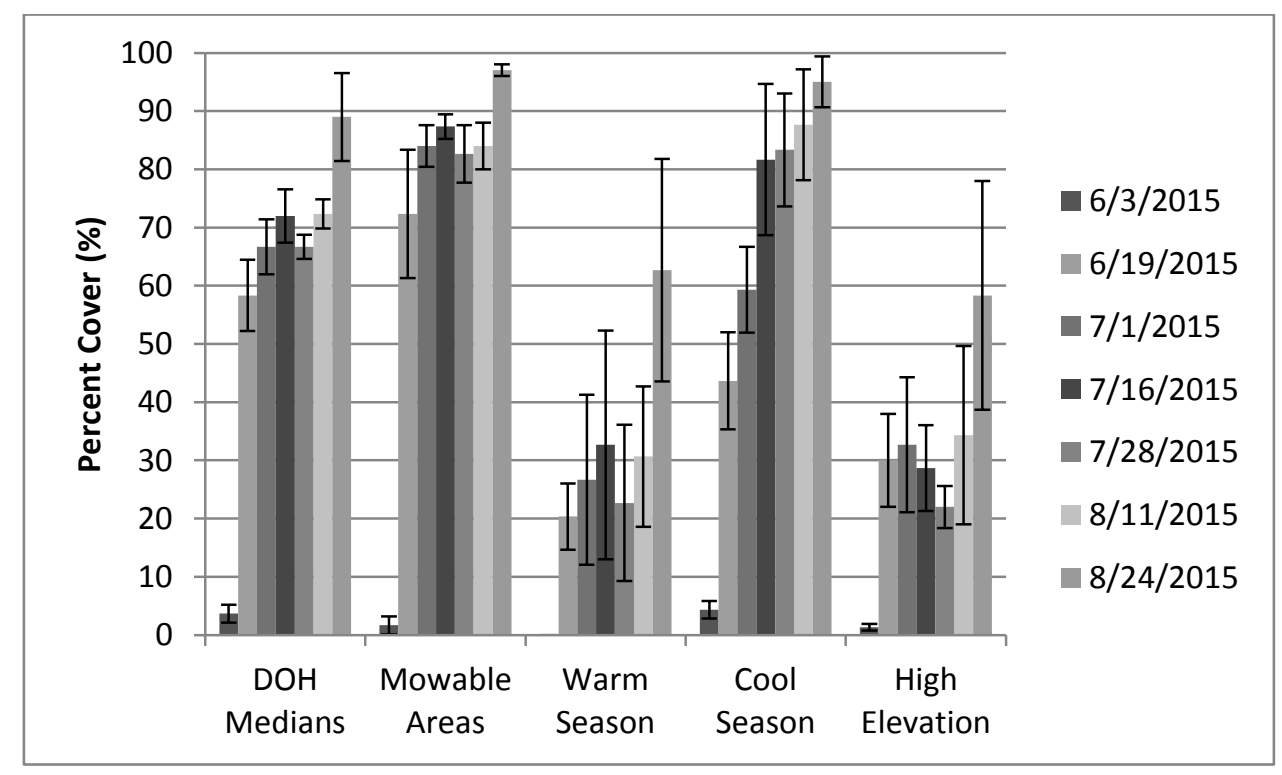

Figure 67: Objective 2 Percent Cover

Percent cover by species was examined 51 days after planting. Warm Season and High Elevation had the heights percent of no cover at $48 \%$ and $46 \%$. They also had the highest percent of not planted species at $8 \%$ and $6 \%$ predominately composed of red clover (Trifolium pratense). DOH Medians, Mowable Areas, and Cool Season mixtures all had a percent cover of planted species in the $80 \%$ range. The majority of cover for these mixtures was the legume species. The results can be seen in Figure 68 and the data can be seen in Appendix 7.3. 


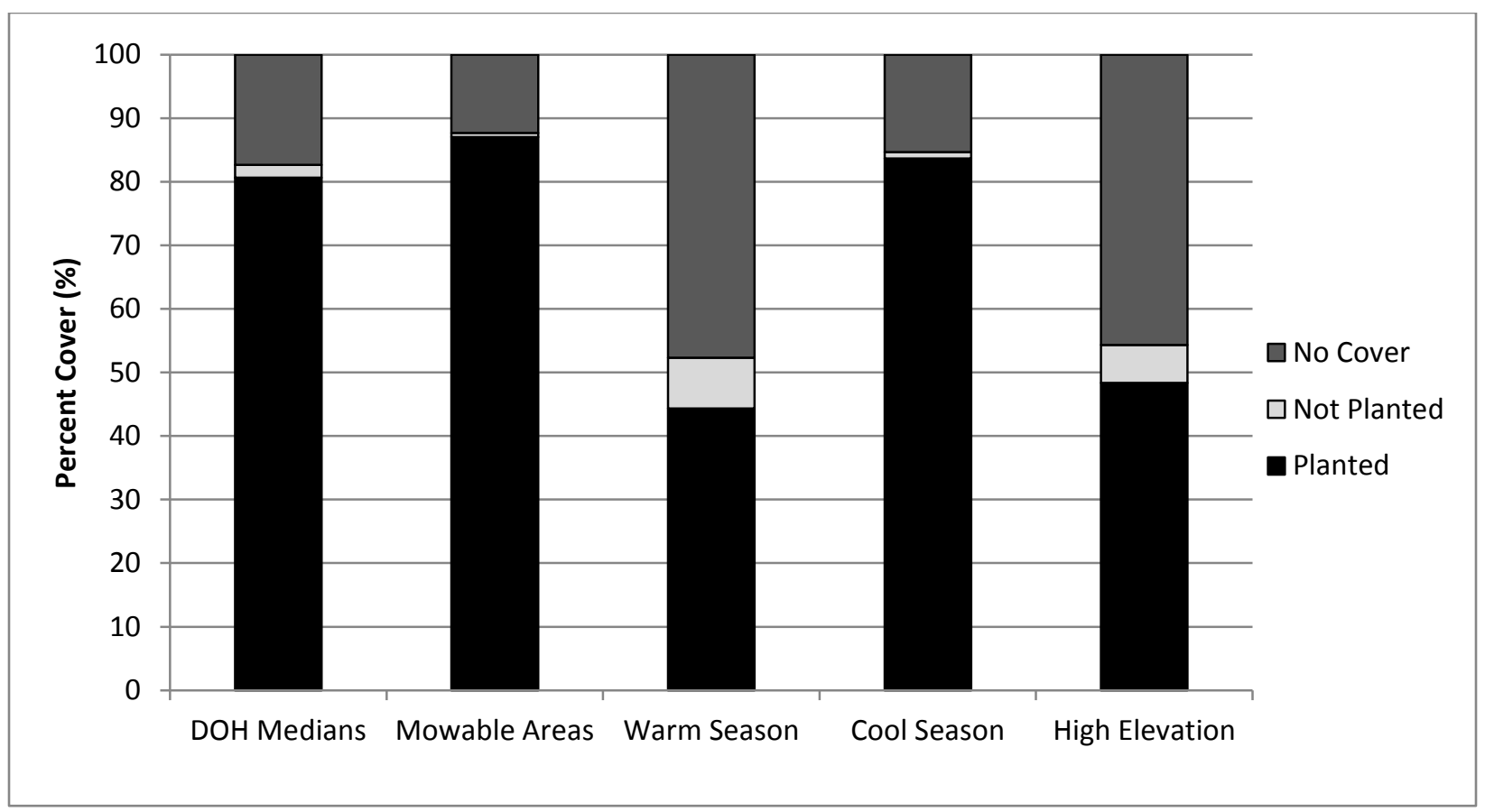

Figure 68: Objective 2 Percent Cover by Species at 51 Days

At 90 days after planting, the percent cover by species was similar in comparison to examination at 51 days. Warm Season and High Elevation had the highest percent of no cover at 55\% and 50\%. Warm Season, Cool Season, and High Elevation had the highest percent of not planted species at $25 \%, 27 \%$, and $32 \%$ predominately composed of red clover (Trifolium pratense) found heavily around the test site. DOH Medians and Mowable Areas mixtures had the highest percent planted species at $86 \%$ and $90 \%$ and the lowest not planted percent at $1 \%$ and $0 \%$. The majority of cover for these two mixtures was the legume species in the mixtures. The results can be seen in Figure 69 and the data can be seen in Appendix 7.3. 


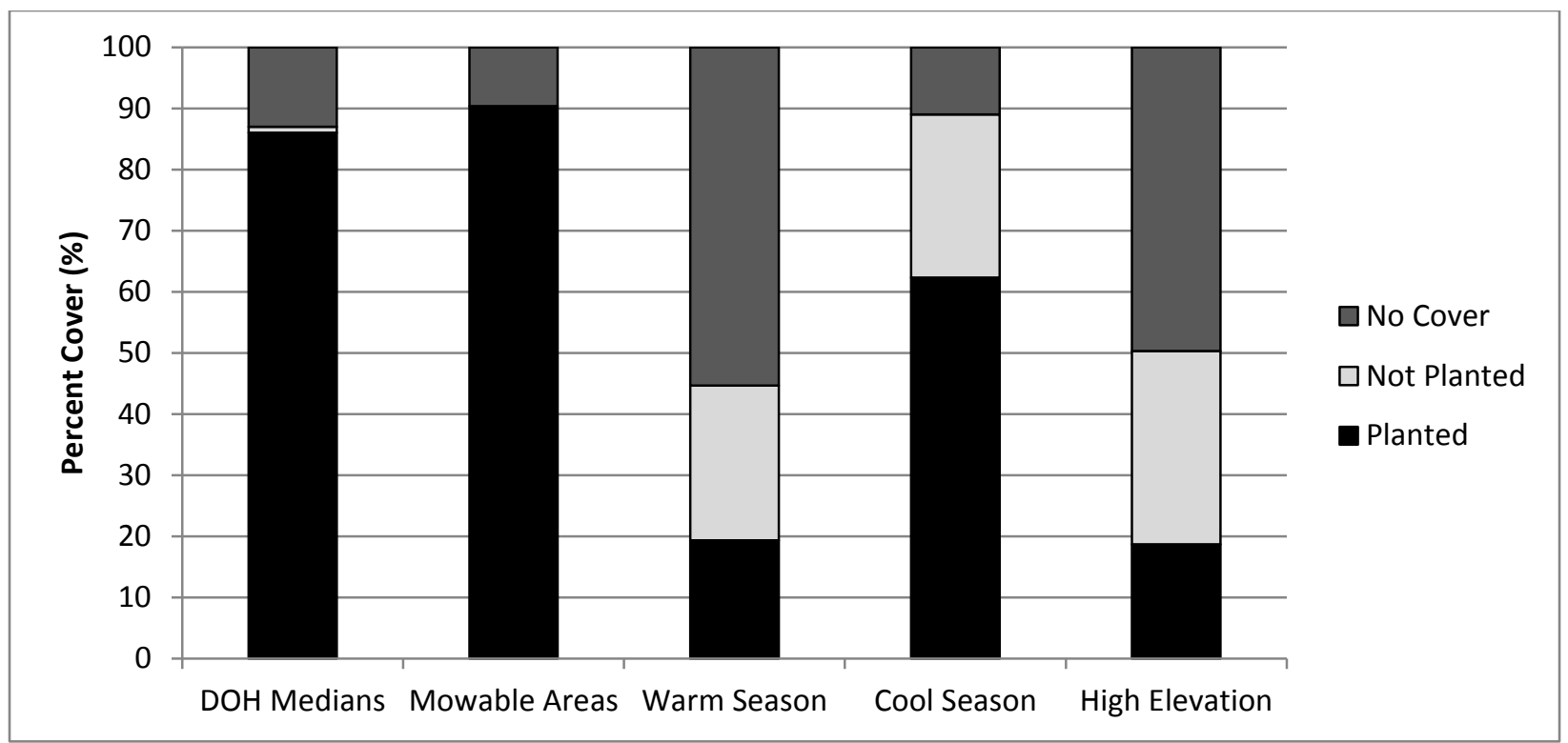

Figure 69: Objective 2 Percent Cover by Species at 90 Days

A one-way ANOVA analysis on percent cover after eight days from planting indicated there was a significant difference between the seed mixtures with a p-value of 0.0079 seen in Table 29. The High Elevation, Mowable Areas and Warm Season mixtures had a slower germination than the Cool Season and DOH Medians mixtures seen in Figure 70. The native warm season mixtures, High Elevation and Warm Season, were expected to germinate slower due to the nature of native warm season species (Salon and Miller 2012), but the Mowable Areas, non-native mixture, was not anticipated to be slower because it contains fast germinating and establishing species like the Cool Season mixture.

Table 29: Objective 2 Eight Day One-way ANOVA Statistics

\begin{tabular}{lccccc}
\hline Source & DF & $\begin{array}{c}\text { Sum of } \\
\text { Squares }\end{array}$ & $\begin{array}{c}\text { Mean } \\
\text { Square }\end{array}$ & F Ratio & Prob $>$ F \\
\hline Treatment & 4 & 37.733333 & 9.43333 & 6.4318 & $0.0079^{*}$ \\
Error & 10 & 14.666667 & 1.46667 & & \\
C. Total & 14 & 52.400000 & & & \\
\hline
\end{tabular}




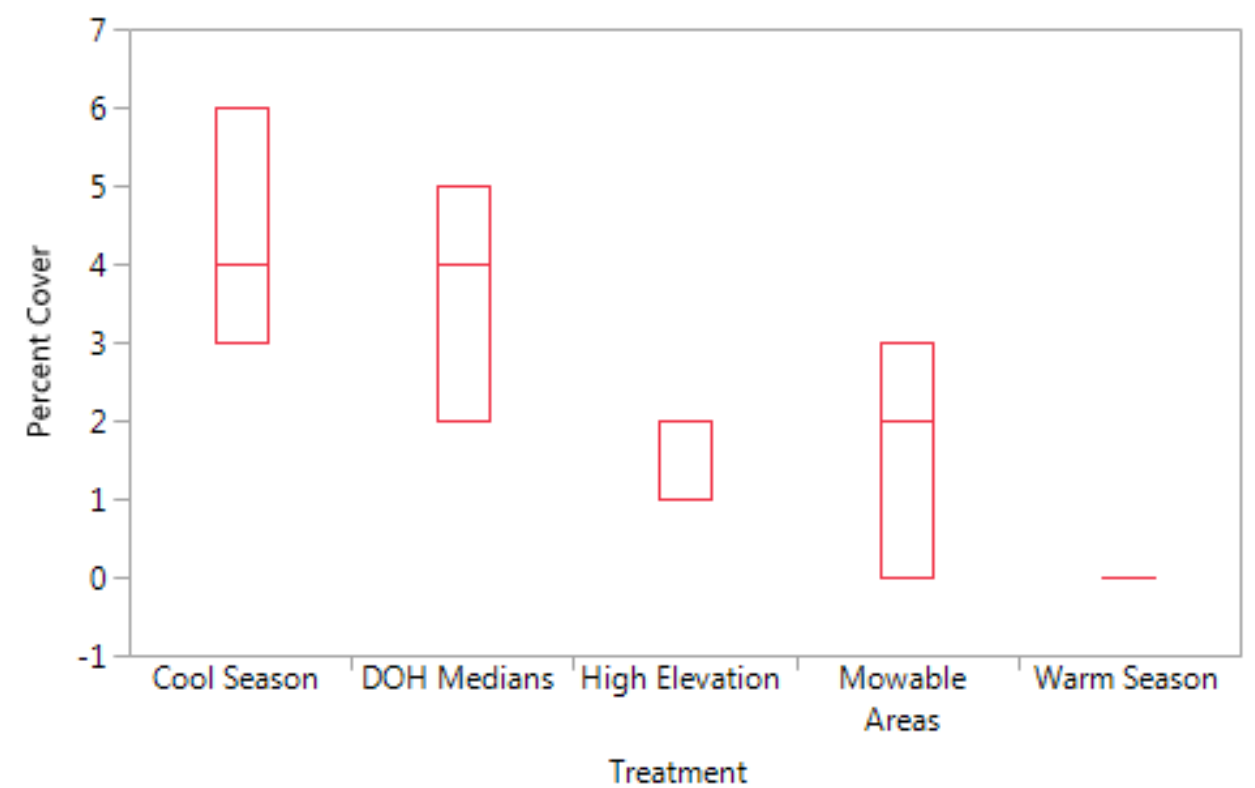

Figure 70: Objective 2 Eight Day One-way ANOVA Graph; n=3; 25\%-75\% (box); median (50\% line in box)

After eight days from planting, a paired student's $t$ test was also performed on the percent cover data. A table of paired student's $t$ test $p$-values and a connecting letters report can be seen in Table 30 and 31. The test resulted in Cool Season and DOH Medians compared to Warm Season mixture and Cool Season and DOH Medians compared to High Elevation mixture to be statistically significant with higher mean percent cover values. The test also indicated that Cool Season had a higher mean value of percent cover compared to the examined Mowable Areas mixture.

Table 30: Objective 2 Eight Day Paired Student's t Test p-Values

\begin{tabular}{llc}
\hline Level & - Level & p-Value \\
\hline Cool Season & Warm Season & $0.0014^{*}$ \\
DOH Medians & Warm Season & $0.0041^{*}$ \\
Cool Season & High Elevation & $0.0126^{*}$ \\
Cool Season & Mowable Areas & $0.0224^{*}$ \\
DOH Medians & High Elevation & $0.0400^{*}$ \\
DOH Medians & Mowable Areas & 0.0707 \\
Mowable Areas & Warm Season & 0.1228 \\
High Elevation & Warm Season & 0.2073 \\
Cool Season & DOH Medians & 0.5155 \\
Mowable Areas & High Elevation & 0.7430 \\
\hline
\end{tabular}


Table 31: Objective 2 Eight Day Connecting Letters Report

\begin{tabular}{lcccc}
\hline Level & & & Mean \\
\hline Cool Season & A & & & 4 \\
DOH Medians & A & B & & 3 \\
Mowable & & B & C & 1 \\
Areas & & & \\
High Elevation & & C & 1 \\
Warm Season & & C & 0 \\
\hline
\end{tabular}

Concluding the end of the study at ninety days of growth, a one-way ANOVA analysis on percent cover indicated a significant difference between the native warm season and non-native cool season seed mixtures with a p-value of 0.0001 seen in Table 32. The High Elevation and Warm Season mixtures were unable to perform as well as the Cool Season, DOH Medians, and Mowable Areas mixtures located in Figure 71.

Table 32: Objective 2 Ninety Day One-way ANOVA Statistics

\begin{tabular}{lccccc}
\hline Source & DF & $\begin{array}{c}\text { Sum of } \\
\text { Squares }\end{array}$ & $\begin{array}{c}\text { Mean } \\
\text { Square }\end{array}$ & F Ratio & Prob $>$ F \\
\hline Treatment & 4 & 36964.25 & 9241.06 & 13.6074 & $<.0001^{*}$ \\
Error & 100 & 67912.00 & 679.12 & & \\
C. Total & 104 & 104876.25 & & & \\
\hline
\end{tabular}

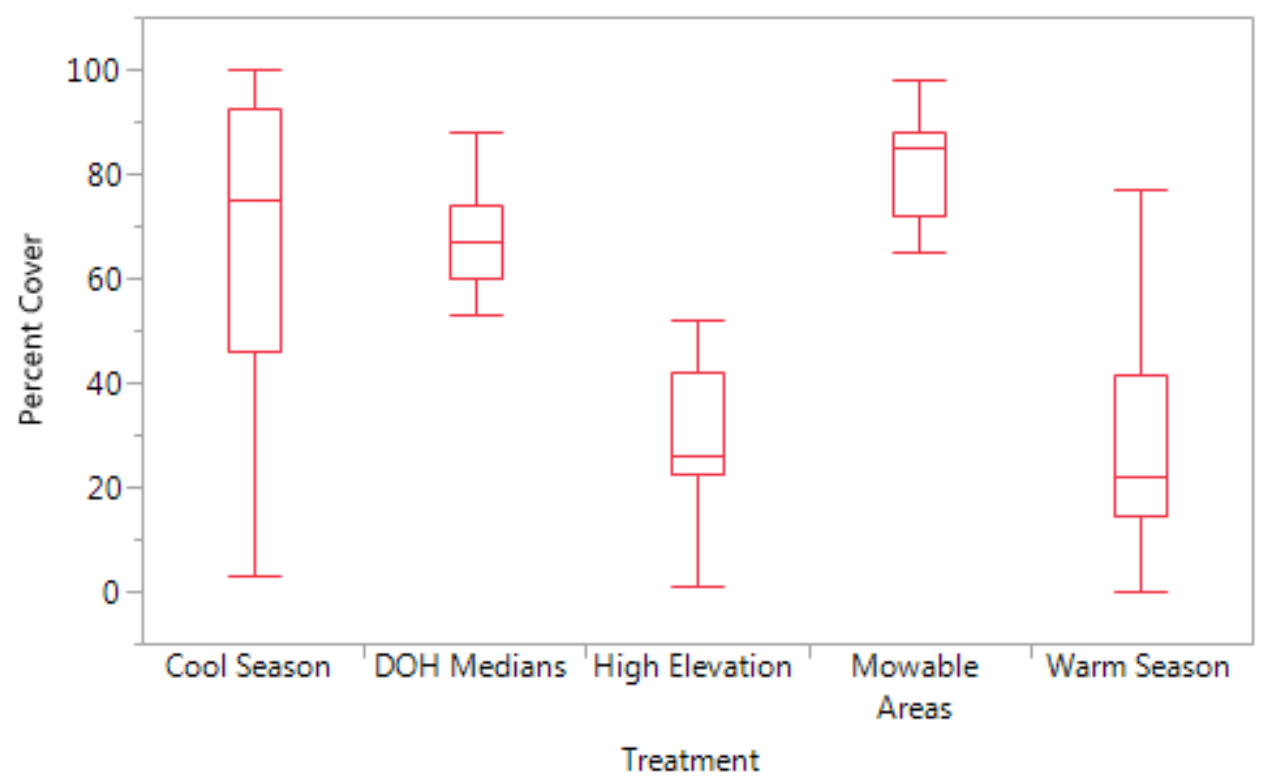

Figure 71: Objective 2 Ninety Day One-way ANOVA Graph; min and max (whisker); 25\%-75\% (box); median ( $50 \%$ line in box) 


\subsubsection{Monitoring Data}

The precipitation and mean ambient air temperature were monitored over the course of the study and can be viewed in Figure 72. The months of June saw the highest cumulative precipitation at $6.9 \mathrm{in} .(17.5 \mathrm{~cm})$. A total accumulation of $14.6 \mathrm{in}(37 \mathrm{~cm})$ were documented over the course of the study. June 27,2015 saw the highest daily precipitation at $1.8 \mathrm{in} .(4.6 \mathrm{~cm})$. The highest monthly mean ambient air temperature was for the month of July at $68.1^{\circ} \mathrm{F}\left(20.0^{\circ} \mathrm{C}\right)$ which also saw the highest mean daily temperature at $74.9^{\circ} \mathrm{F}\left(23.8^{\circ} \mathrm{C}\right)$. The lowest mean temperature occurred May 3,2015 at $51.3^{\circ} \mathrm{F}\left(10.7^{\circ} \mathrm{C}\right)$.

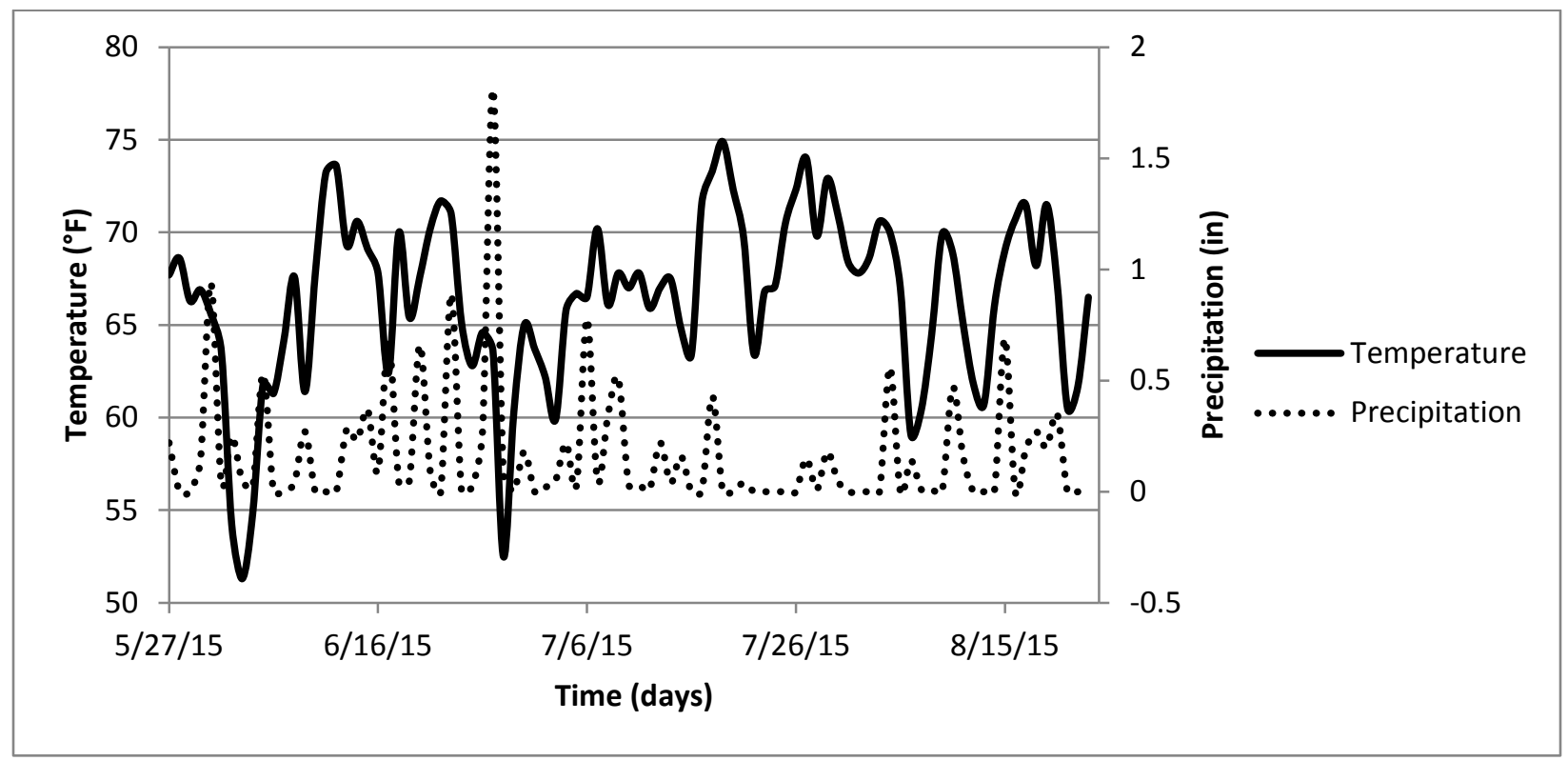

Figure 72: Objective 2 Weather Conditions

The mean soil temperature and volumetric water content was monitored over the course of the study and can be viewed in Figure 73. The mean soil temperatures for the month of June, July, and August were $66.6^{\circ} \mathrm{F}, 71.2^{\circ} \mathrm{F}$, and $70.4^{\circ} \mathrm{F}\left(19.2^{\circ} \mathrm{C}, 21.8^{\circ} \mathrm{C}\right.$, and $\left.21.3^{\circ} \mathrm{C}\right)$. As previously noted, black plastic was used prior to planting which caused the initial soil temperature to be high. The highest soil temperature was documented on June 27,2015 at $77.7^{\circ} \mathrm{F}\left(25.4^{\circ} \mathrm{C}\right)$ under normal vegetation conditions. 
The low soil temperatures could have negatively affected the germination of the Warm Season and High Elevation mixtures. Those mixtures are composed of primarily warm season native species which prefer warm soil temperatures during their growth cycle. The Warm Season and High Elevation species preferred a soil temperature around $70^{\circ} \mathrm{F}\left(21^{\circ} \mathrm{C}\right)$ (Royal Botanic Gardens, Kew 2015). The low initial temperatures could have kept the seeds in dormancy resulting in later germination in the summer season.

Volumetric water content typically saw spikes when rain events occurred, but quickly decreased below $10 \%$ after the events. The dramatic decrease in the soil volumetric water content was due to the properties of the soil. The site had a shallow soil surface with underlying bed rock. The shallow soil surface was composed of large aggregates from a previous road bed which likely resulted in quick infiltration of the rain water to the underlying bedrock. The rapid infiltration would then indicate the large change in volumetric water content seen in Figure 73.

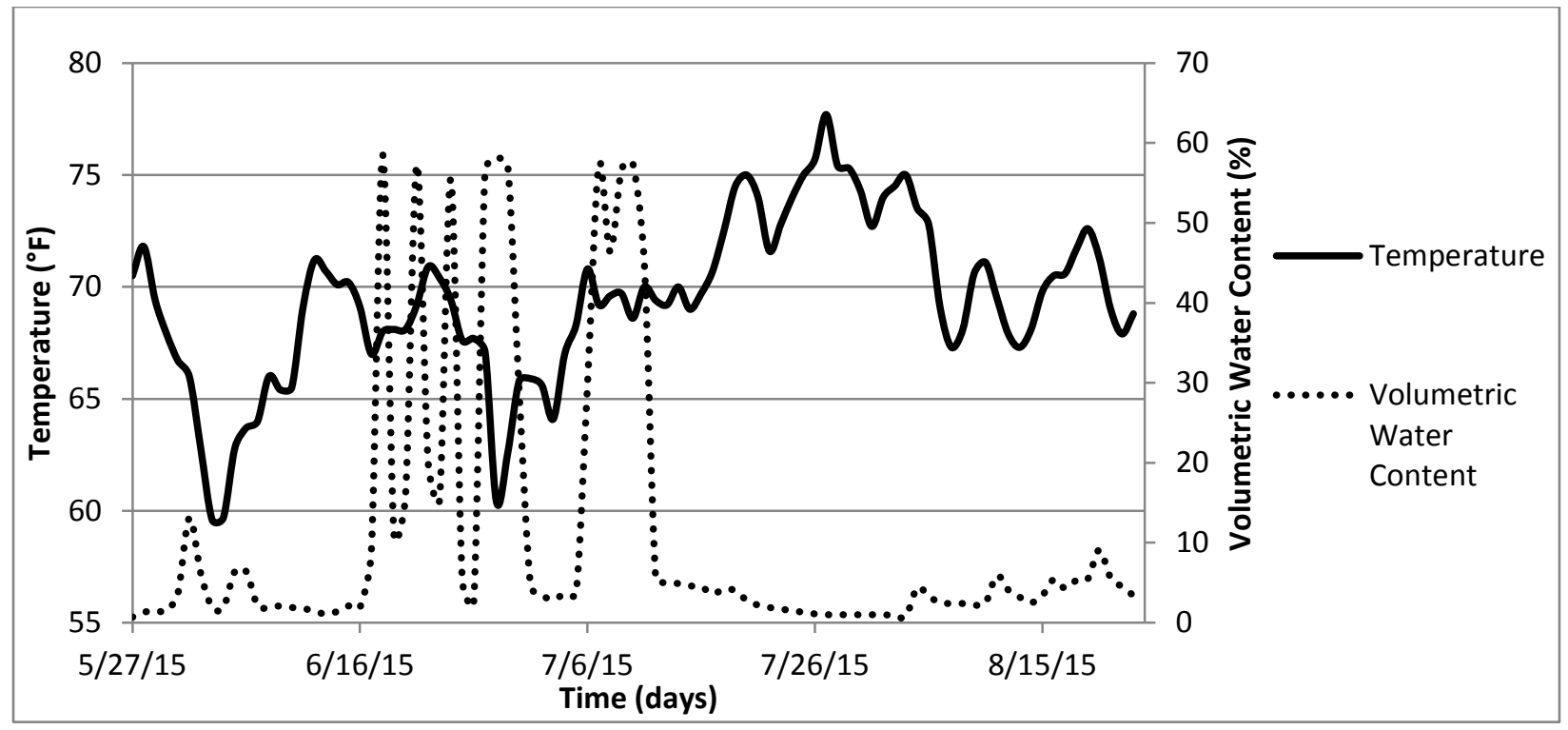

Figure 73: Objective 2 Soil Characteristics

Electrical conductivity of the soil was monitored with a soil sensor and can be seen graphed in Figure 74 . The change in electrical conductivity closely resembles the change in the soil volumetric water content seen in Figure 73. 


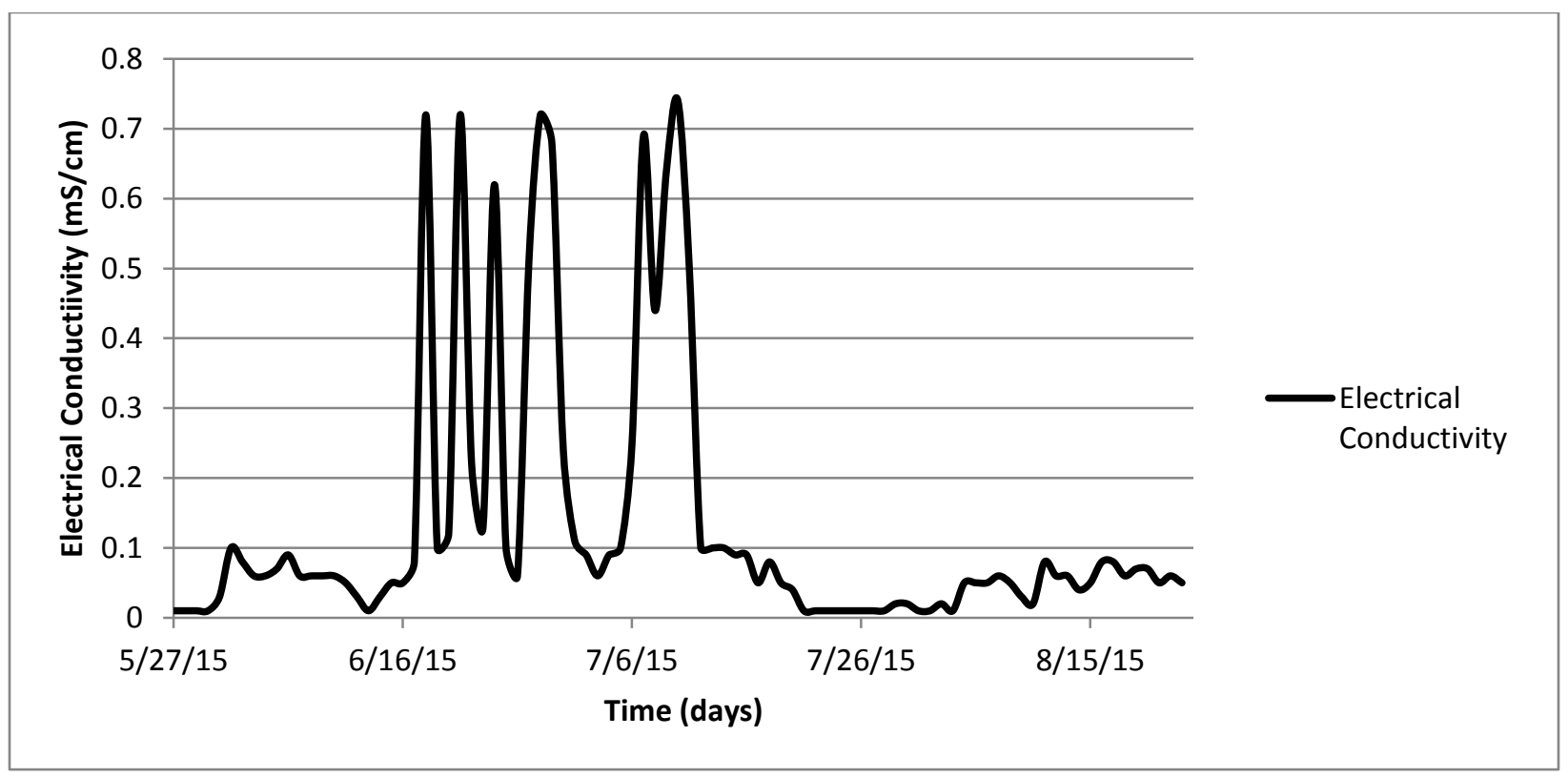

Figure 74: Objective 2 Mean Electrical Conductivity

The initial site compaction data in Table 33 shows severe compaction conditions for the top 9 in. which would limit or stunt root development. After site preparation, the first 3 in. improved to a rank of "Red" that would provide poor compaction conditions. The remaining 6 in. improved slightly, but were still over compacted. The compaction ranking and classification can be seen in Table 28. An explanation of rank and scale can be seen in Section 4.4.1.2.

Table 33: CH-2 Compaction Data

\begin{tabular}{lcccccc}
\hline $\begin{array}{c}\text { Penetrometer } \\
\text { Depth } \\
\text { Markings }\end{array}$ & $\begin{array}{l}\text { Before Site } \\
\text { Preparation }\end{array}$ & \multicolumn{5}{c}{ After Site Preparation } \\
\cline { 2 - 7 } & $\mathrm{CH}-2$ & $\begin{array}{c}\text { DOH } \\
\text { Medians }\end{array}$ & $\begin{array}{c}\text { Mowable } \\
\text { Areas }\end{array}$ & $\begin{array}{c}\text { Warm } \\
\text { Season }\end{array}$ & $\begin{array}{c}\text { Cool } \\
\text { Season }\end{array}$ & $\begin{array}{c}\text { High } \\
\text { Elevation }\end{array}$ \\
3" Color: & 0 & 0.7 & 0.8 & 0.8 & 0.7 & 0.8 \\
6" Color: & 0 & 0.3 & 0.5 & 0.3 & 0.2 & 0.3 \\
9" Color: & 0 & 0.2 & 0.2 & 0.0 & 0.0 & 0.0 \\
\hline
\end{tabular}




\subsubsection{Objective 3: Examining seedbed preparation techniques}

\subsubsection{Vegetation Measurement}

The percent covers of twelve treatments were examined over a 90 day period. All treatments were able to achieve $70 \%$ vegetation cover by 51 days after planting. The percent cover results can be seen in Figure 75 and the data can be seen in Appendix 7.4.

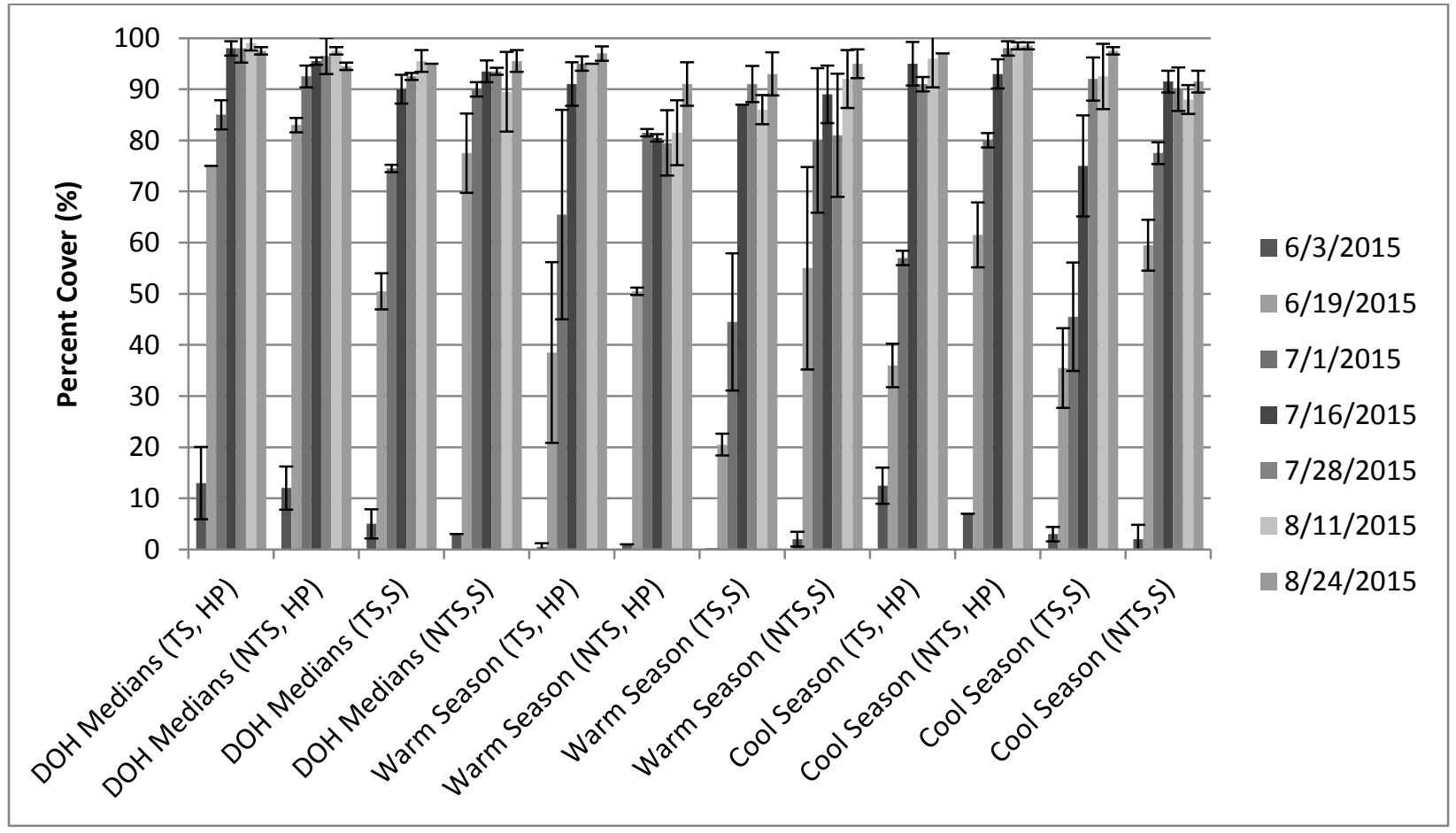

Figure 75: Objective 3 Percent Cover (TS= topsoil, NTS, no topsoil, HP= hydraulic erosion control product, $\mathrm{S}=$ straw)

At 51 days, percent cover by species was examined. The treatments containing $\mathrm{DOH}$ Medians and Warm Season seed mixtures showed treatments with topsoil with a higher percent of not planted species then no topsoil treatments. The high percent of not planted species was likely due to the topsoil containing high levels of seed that was not destroyed or removed by the provider. The most prevalent not planted species was barnyardgrass (Echinochloa crus-galli) which is not native to West Virginia. The treatments with the Cool Season seed mixture had similar levels of not planted species in both topsoil and no topsoil treatments.

The topsoil treatments utilizing the Warm Season seed mixture had the highest percent of not planted species at $36 \%$ and $53 \%$. Another noticeable difference between all treatments 
was the fact straw had higher levels of not planted species than the comparative hydraulic erosion control product treatments. Also, the hydraulic erosion control product treatments had higher levels of planted species then corresponding straw treatments in all cases. The results can be seen in Figure 76 and the data can be seen in Appendix 7.4.

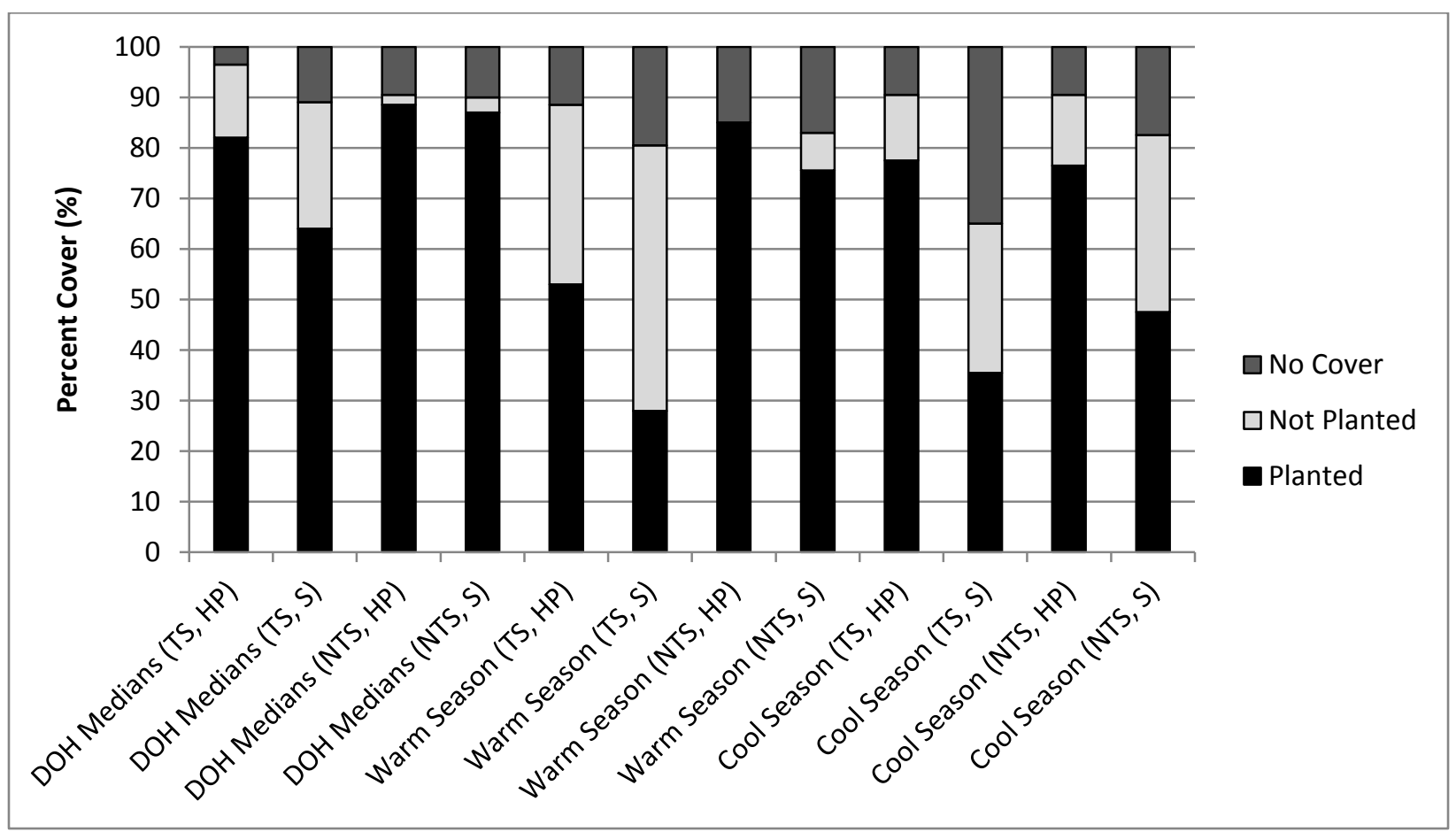

Figure 76: Objective 3 Percent by Species at 51 Days (TS= topsoil, NTS, no topsoil, HP= hydraulic erosion control product, $\mathrm{S}=$ straw)

At 90 days, percent cover by species was examined and indicated high levels of not planted species in treatments with topsoil. The reason behind a high percent of not planted species was the same as previously discussed at 51 days examination. The not planted cover ranged from $41 \%-84 \%$ for treatments with topsoil and only $0 \%-17 \%$ in no topsoil treatments. The topsoil affect influenced WVDOH Median treatments the greatest with a planted cover at $17 \%$ for treatment with topsoil and $90 \%-97 \%$ planted cover for no topsoil treatments. The other treatments did see large differences similar to the WVDOH Median treatments. The results can be seen in Figure 77 and the data can be seen in Appendix 7.4. 


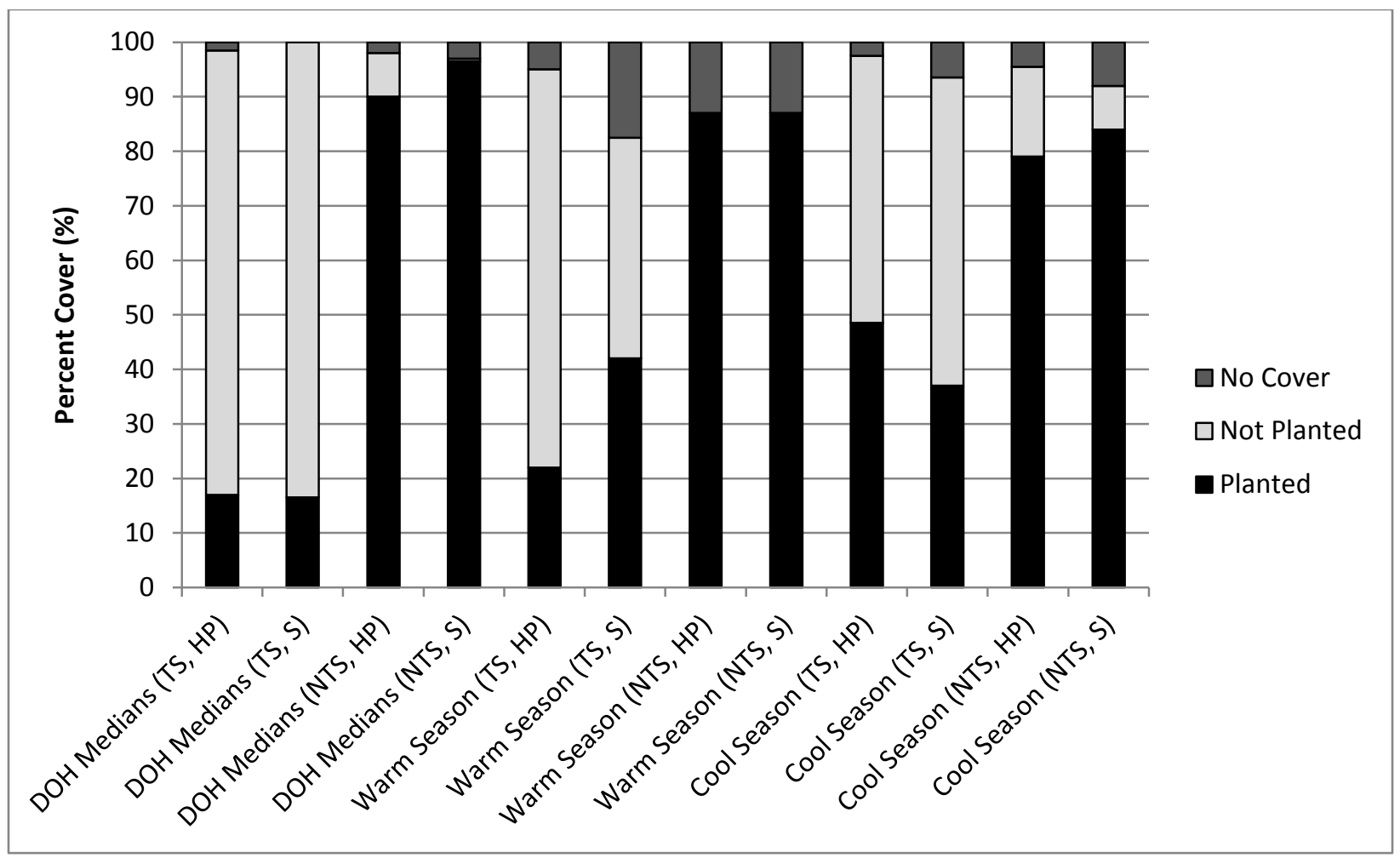

Figure 77: Objective 3 Percent by Species at 90 Days (TS= topsoil, NTS, no topsoil, HP= hydraulic erosion control product, $\mathrm{S}=$ straw)

A one-way blocked ANOVA analysis on percent cover by seed mixture after eight days from planting was performed and can be seen in Table 34. The ANOVA found a p-value of 0.0015 for the seed mixtures and 0.0132 for the treatments. There was a statistical significance for percent cover by seed mixtures and treatments. The Warm Season seed mixture did not perform to the same level as the Cool Season and DOH Medians mixtures. Again, the native Warm Season mixture were characterized by species that have a slower germination rate compared to non-native cool season species (Salon and Miller 2012) in the Cool Season and DOH Medians mixtures seen in Figure 78.

Table 34: Objective 3 Eight Day One-way Blocked ANOVA of Percent Cover by Seed Mixture Statistics

\begin{tabular}{lccccc}
\hline Source & DF & $\begin{array}{c}\text { Sum of } \\
\text { Squares }\end{array}$ & $\begin{array}{c}\text { Mean } \\
\text { Square }\end{array}$ & F Ratio & Prob > F \\
\hline $\begin{array}{l}\text { Seed } \\
\text { Mixture }\end{array}$ & 2 & 230.58333 & 115.292 & 9.4869 & $0.0015^{*}$ \\
Treatment & 3 & 172.50000 & 57.500 & 4.7314 & $0.0132^{*}$ \\
Error & 18 & 218.75000 & 12.153 & & \\
C. Total & 23 & 621.83333 & & & \\
\hline
\end{tabular}




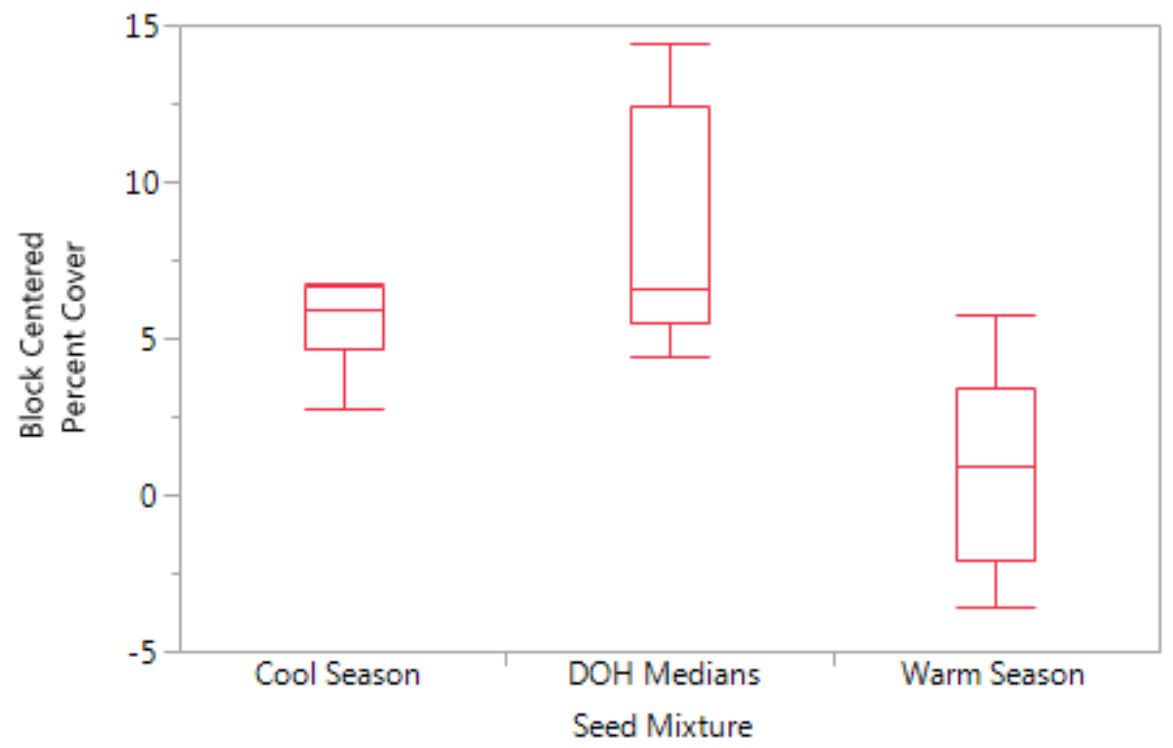

Figure 78: Objective 3 Eight Day One-way Blocked ANOVA of Percent Cover by Seed Mixture Graph; min and max (whisker); $25 \%-75 \%$ (box); median ( $50 \%$ line in box)

The paired student's t test p-values and connecting letters report in Table 35 and 36 shows that DOH Medians and Cool Season mixtures are significantly similar based on close mean values and a p-value of 0.2385 . However, DOH Medians and Cool Season percent mean covers are not correlated to the Warm Season mixture based on mean percent covers.

Table 35: Objective 3 Paired Student's t Test p-Values for Percent Cover by Seed Mixture Analysis

\begin{tabular}{lll}
\hline Level & - Level & p-Value \\
\hline DOH Medians & Warm Season & $0.0005^{*}$ \\
Cool Season & Warm Season & $0.0075^{*}$ \\
DOH Medians & Cool Season & 0.2385 \\
\hline
\end{tabular}

Table 36: Objective 3 Connecting Letters Report for Percent Cover by Seed Mixture Analysis

\begin{tabular}{|c|c|c|}
\hline Level & & Mean \\
\hline $\begin{array}{l}\text { DOH } \\
\text { Medians }\end{array}$ & A & 8 \\
\hline Cool Season & A & 6 \\
\hline $\begin{array}{l}\text { Warm } \\
\text { Season }\end{array}$ & & 0.9 \\
\hline
\end{tabular}

A one-way blocked ANOVA analysis for percent cover by treatment after eight days from planting was then performed and can be seen in Table 37. The ANOVA p-values were the same as in Table 34 and can be seen in Table 37. The significance comes from the graph seen in 
Figure 79. Treatments with HECP (HP) had significantly higher percent covers compared to treatments with straw (S).

Table 37: Objective 3 Eight Day One-way Blocked ANOVA of Percent Cover by Treatment Statistics

\begin{tabular}{lrrrrr}
\hline Source & DF & $\begin{array}{r}\text { Sum of } \\
\text { Squares }\end{array}$ & $\begin{array}{r}\text { Mean } \\
\text { Square }\end{array}$ & F Ratio & Prob > F \\
\hline Treatment & 3 & 172.50000 & 57.500 & 4.7314 & $0.0132^{*}$ \\
$\begin{array}{l}\text { Seed } \\
\text { Mixture }\end{array}$ & 2 & 230.58333 & 115.292 & 9.4869 & $0.0015^{*}$ \\
Error & 18 & 218.75000 & 12.153 & & \\
C. Total & 23 & 621.83333 & & & \\
\hline
\end{tabular}

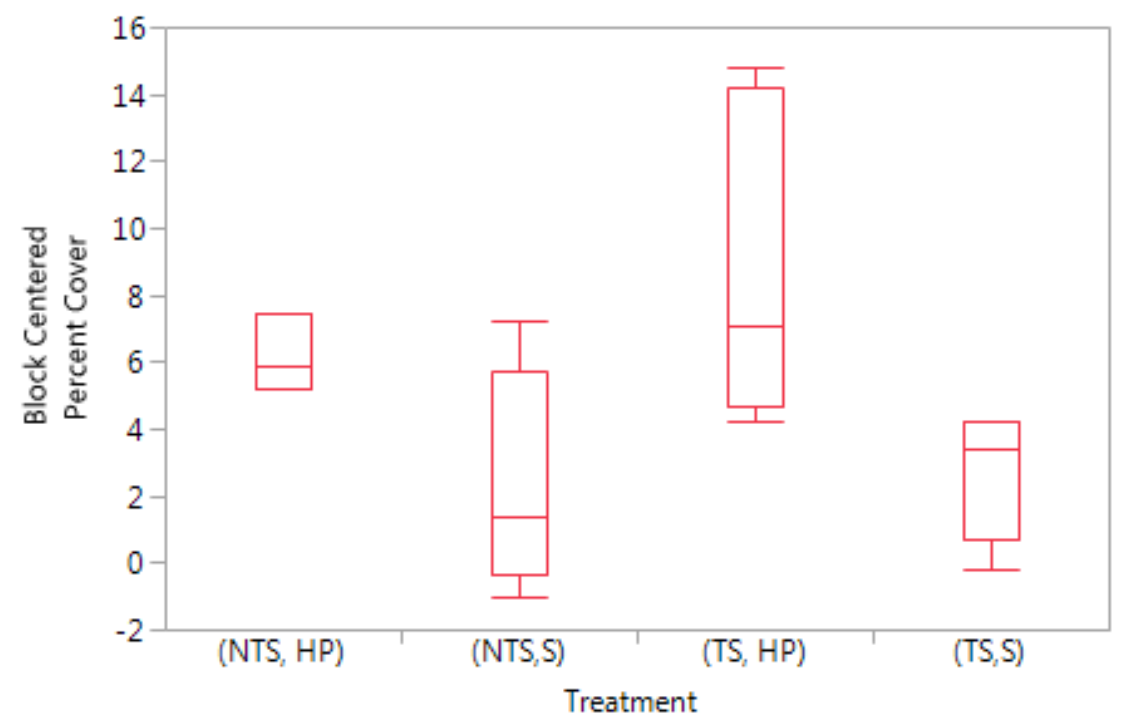

Figure 79: Objective 3 Eight Day One-way Blocked ANOVA of Percent Cover by Treatment Graph; min and max (whisker); $25 \%-75 \%$ (box); median ( $50 \%$ line in box); (TS= topsoil, NTS, no topsoil, HP= hydraulic erosion control product, $\mathrm{S}=$ straw)

The paired student's t test p-values and connecting letters report for percent cover are illustrated in Table 38 and 39. The report shows that treatments with topsoil and HECP (TS, HP) had a significant higher mean percent cover and statistical significance compared to no topsoil and straw (NTS, S) and topsoil and straw (TS, S) treatments with corresponding p-values of 0.0056 and 0.0080. No topsoil and HECP (NTS, HP) also had a higher mean percent cover compared to no topsoil and straw (NTS, S) treatments with a significant p-value of 0.0451 . 
Table 38: Objective 3 Paired Student's t Test $p$-Values for Percent Cover by Treatment Analysis

\begin{tabular}{llr}
\hline Level & - Level & p-Value \\
\hline (TS, HP) & (NTS,S) & $0.0056^{*}$ \\
(TS, HP) & (TS,S) & $0.0080^{*}$ \\
(NTS, HP) & (NTS,S) & $0.0451^{*}$ \\
(NTS, HP) & (TS,S) & 0.0623 \\
(TS, HP) & (NTS, HP) & 0.3335 \\
(TS,S) & (NTS,S) & 0.8703 \\
\hline
\end{tabular}

Table 39: Objective 3 Connecting Letters Report for Percent Cover by Treatment Analysis

\begin{tabular}{lrrr}
\hline Level & & & Mean \\
\hline (TS, HP) & A & & 8.7 \\
(NTS, HP) & A & B & 6.7 \\
(TS,S) & B C & 2.7 \\
(NTS,S) & & C & 2.3 \\
\hline
\end{tabular}

A one-way blocked ANOVA analysis on percent cover was also performed for 90 days after planting and can be seen in Table 40. The ANOVA found a $p$ value of 0.1814 for the seed mixtures and 0.5110 for the treatments. There was no statistical difference between the various seed mixtures and treatments. The Warm Season mixture was able to perform to the same level as the Cool Season and $\mathrm{DOH}$ Medians mixtures in long term establishment and can be seen in Figure 80. In Figure 81, all treatments were similar and no statistical difference was found in Table 40.

Table 40: Objective 3 Ninety Day One-way ANOVA Statistics

\begin{tabular}{lccccc}
\hline Source & DF & $\begin{array}{c}\text { Sum of } \\
\text { Squares }\end{array}$ & $\begin{array}{c}\text { Mean } \\
\text { Square }\end{array}$ & F Ratio & Prob $>$ F \\
\hline Seed & 2 & 3608.68 & 1804.34 & 1.7253 & 0.1814 \\
Mixture & & & & & \\
Treatment & 3 & 2423.49 & 807.83 & 0.7724 & 0.5110 \\
Error & 162 & 169423.20 & 1045.82 & & \\
C. Total & 167 & 175455.38 & & & \\
\hline
\end{tabular}




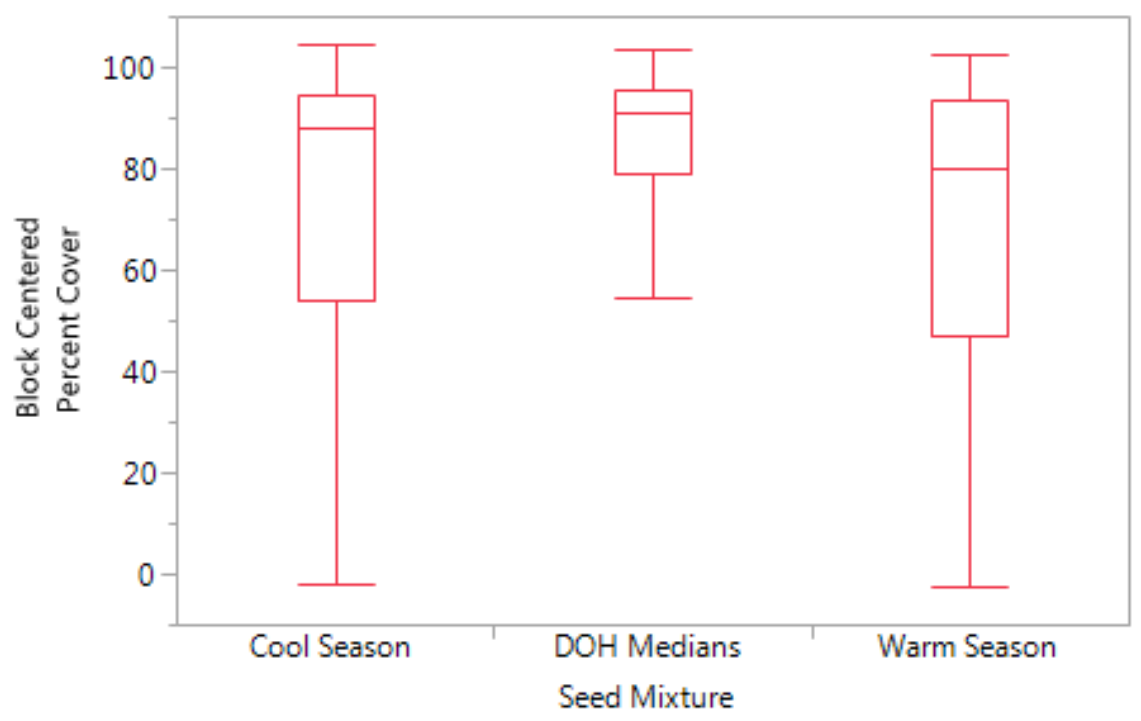

Figure 80: Objective 3 Ninety Day One-way Blocked ANOVA of Percent Cover by Seed Mixture Graph; min and max (whisker); $25 \%-75 \%$ (box); median (50\% line in box)

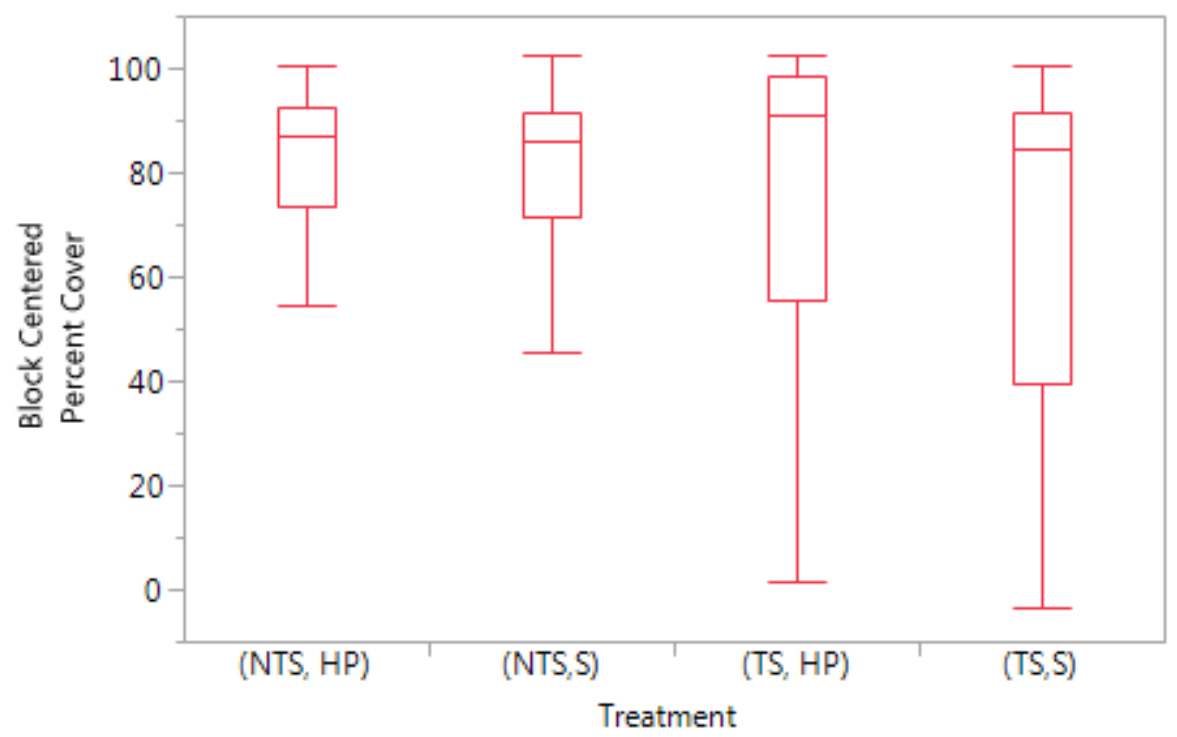

Figure 81: Objective 3 Ninety Day One-way Blocked ANOVA of Percent Cover by Seed Mixture Graph; min and max (whisker); $25 \%-75 \%$ (box); median (50\% line in box)

\subsubsection{Monitoring Data}

The precipitation and mean ambient air temperature were monitored over the course of the study, but data for the first part of July was lost due to a technical error while retrieving the data. The data can be seen in Figure 82. The months of June saw the highest cumulative 
precipitation at $7.9 \mathrm{in} .(20.1 \mathrm{~cm})$. A total accumulation of $13 \mathrm{in} .(33.0 \mathrm{~cm})$ were documented over the course of the study. June 27,2015 saw the highest daily precipitation at $2.51 \mathrm{in} .(6.38 \mathrm{~cm})$.

The highest monthly mean ambient air temperature was for the month of July at $75^{\circ} \mathrm{F}$ $\left(24^{\circ} \mathrm{C}\right)$ which also had the highest mean daily temperature of $81.4^{\circ} \mathrm{F}\left(27.4^{\circ} \mathrm{C}\right)$. The lowest mean temperature occurred May 3,2015 at $55.3^{\circ} \mathrm{F}\left(12.9^{\circ} \mathrm{C}\right)$ which can also be seen in Figure 82.

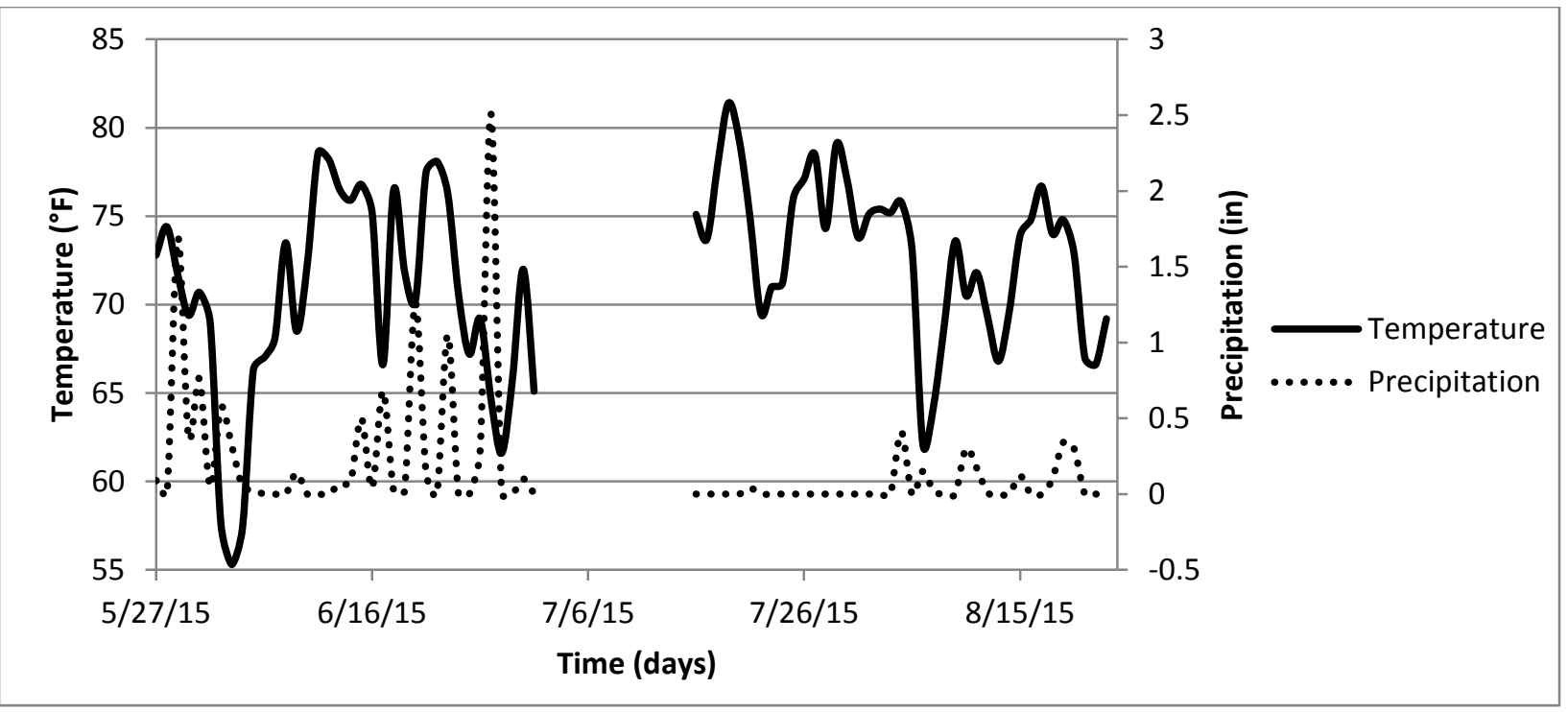

Figure 82: Objective 3 Weather Conditions

Volumetric water content typically saw spikes when rain events occurred indicating valuable rain data was lost at the beginning of July due to the technical error. Examining treatments "TS, HP, 1" and "TS, S, 2" in Figure 83, the two treatments were similar throughout the study. The only difference could be noted during the dryer period after July 18, 2015 where "TS, HP, 1" maintains a volumetric water content around $3 \%$ and "TS, S, 2" stays close to $0 \%$. As for treatments "NTS, HP, 1" and "NTS, S, 1" in Figure 83, "NTS, S, 1" maintains the highest volumetric water content through the course of the study even compared to the previous two discussed treatments. "NTS, HP, 1" had the lowest volumetric water content until the end of the study where "TS, S, 2" dropped to zero when rainfall was limited. 


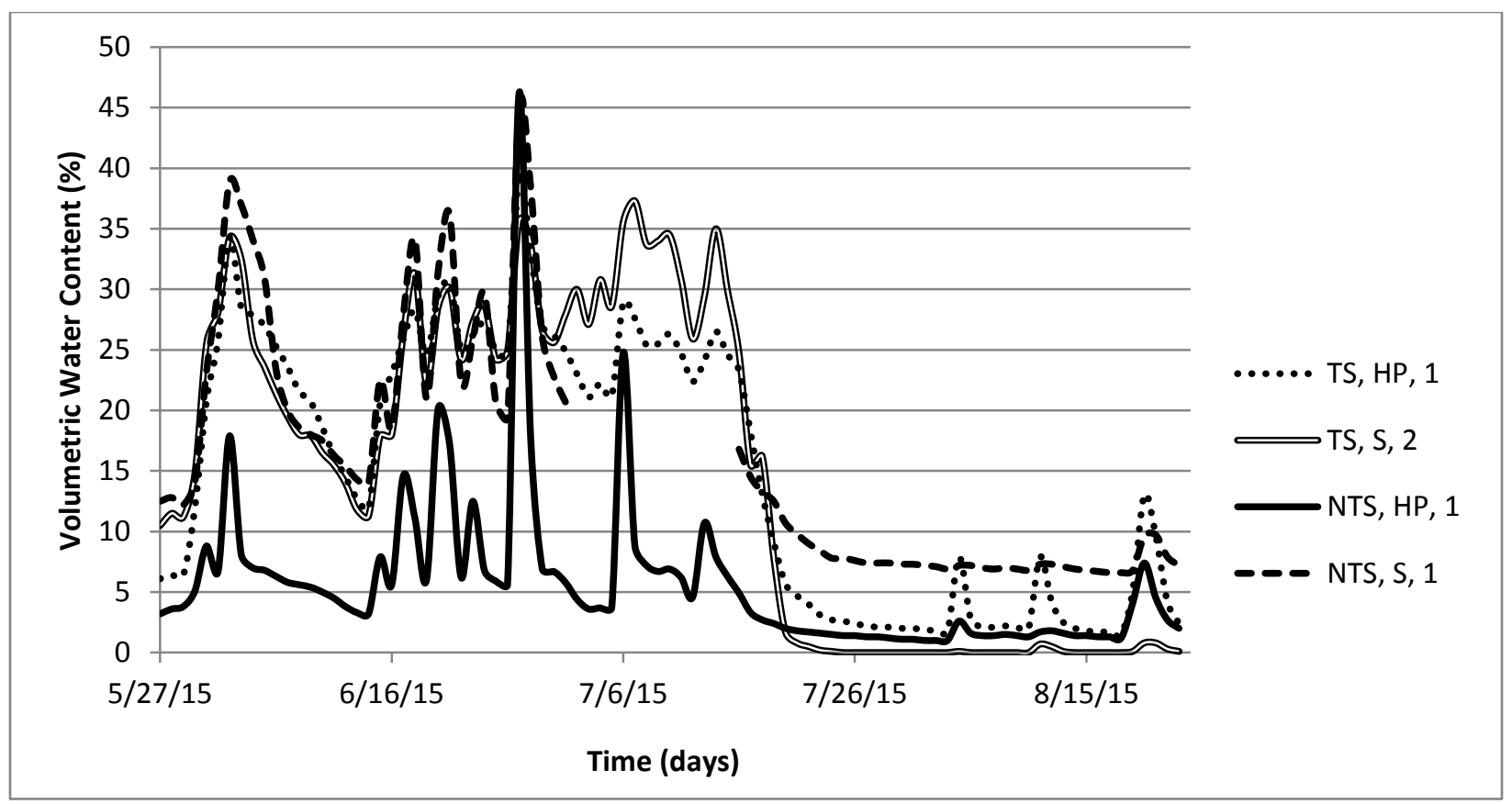

Figure 83: Objective 3 Mean Volumetric Water Content

The mean soil temperature can be viewed in Figure 84. The mean soil temperatures for the month of June, July, and August were $72.7^{\circ} \mathrm{F}, 78.1^{\circ} \mathrm{F}$, and $77.9^{\circ} \mathrm{F}\left(22.6^{\circ} \mathrm{C}^{\circ}, 25.6^{\circ} \mathrm{C}\right.$, and $\left.25.5^{\circ} \mathrm{C}\right)$. A slow rise in temperature could be seen from the start of the study to the first of August. The highest soil temperature was documented on August 1,2015 at $84.2^{\circ} \mathrm{F}\left(29^{\circ} \mathrm{C}\right)$ under normal vegetation conditions. As previously noted, black plastic was used prior to planting which caused the initial soil temperature to be high.

Electrical conductivity of the soil was monitored and can be seen in Figure 84. The change in electrical conductivity closely resembled the change in the soil volumetric water content seen in Figure 83 and precipitation in Figure 82. 


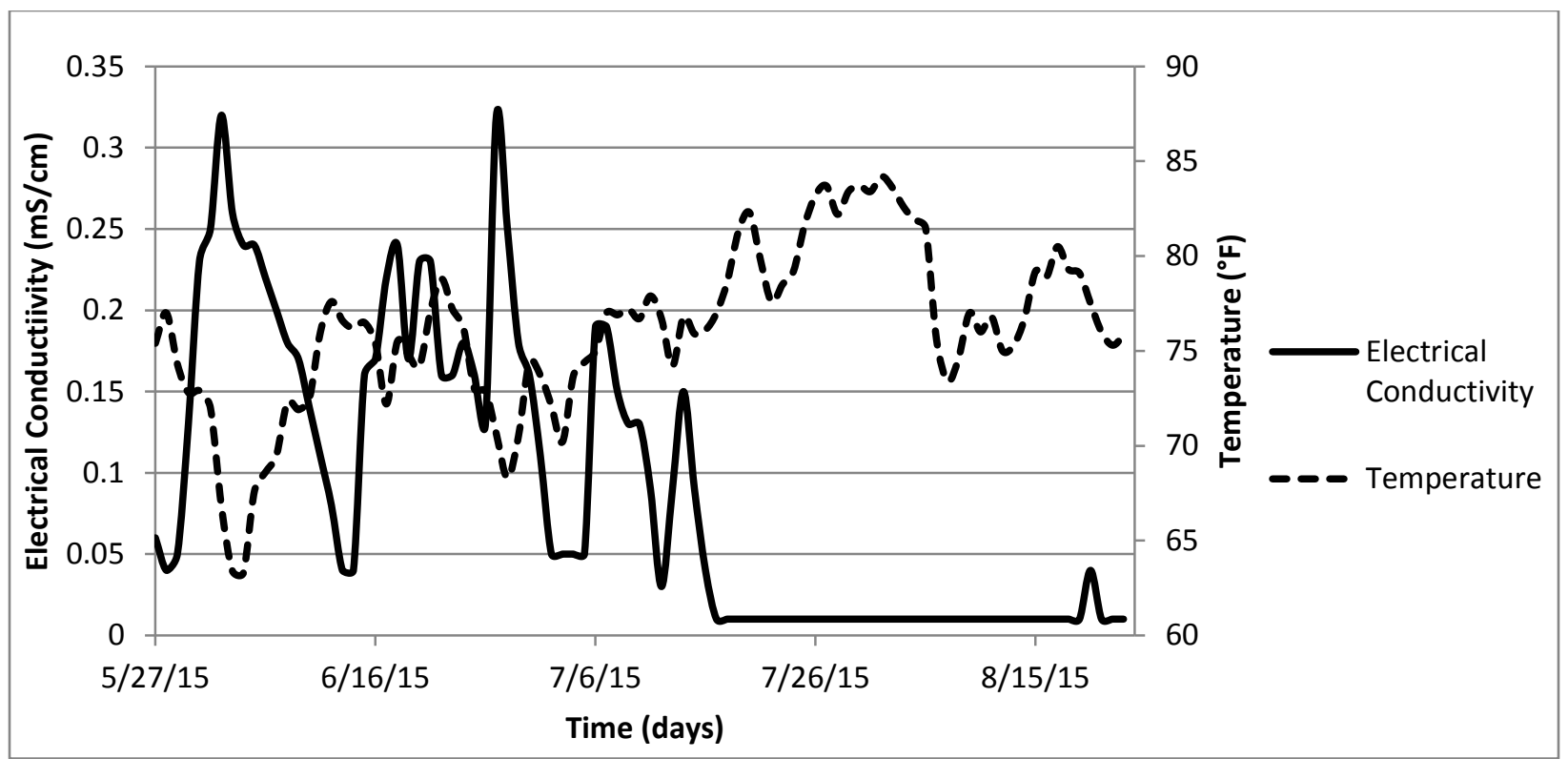

Figure 84: Objective 3 Soil Characteristics

The initial site compaction data in Table 41 again shows severe compaction conditions for the top 9 in. which would hinder root development. After site preparation seen in Table 41, 42, and 43, the first 3 in. for topsoil (TS) subplots improved to a rank of "Green" and would provide optimum compaction conditions. Subplots with no topsoil (NTS) in general ranked lower at "Yellow", but would still demonstrate moderate conditions for root development. At 6 in., half of the subplots displayed poor compaction conditions and the other half had severe compaction problems. At 9 in., all subplots were over compacted at rank "Max". The compaction ranking and classification can be seen in Table 28. An explanation of rank and scale can be seen in Section 4.4.1.2.

Table 41: CH-3 Compaction Data (DOH Medians)

\begin{tabular}{lccccc}
\hline \begin{tabular}{c} 
Penetrometer $\begin{array}{c}\text { Depth } \\
\text { Markings }\end{array}$ \\
\cline { 2 - 6 }
\end{tabular} & $\begin{array}{c}\text { Before Site } \\
\text { Preparation }\end{array}$ & \multicolumn{4}{c}{ After Site Preparation } \\
\cline { 2 - 6 } & $\mathrm{CH}-3$ & $\begin{array}{c}\text { DOH } \\
\text { Medians } \\
\text { (TS, HP) }\end{array}$ & $\begin{array}{c}\text { MOH } \\
\text { Medians } \\
\text { (NTS, HP) }\end{array}$ & $\begin{array}{c}\text { DOH } \\
\text { Medians } \\
(T S, S)\end{array}$ & $\begin{array}{c}\text { MOH } \\
\text { (NTS,S) }\end{array}$ \\
3" Color: & 0 & 2.5 & 2 & 3 & 2.5 \\
6" Color: & 0 & 0.5 & 0.5 & 1 & 0.75 \\
9" Color: & 0 & 0 & 0 & 0.5 & 0.25 \\
\hline
\end{tabular}


Table 42: CH-3 Compaction Data (Warm Season)

\begin{tabular}{|c|c|c|c|c|c|}
\hline \multirow{2}{*}{$\begin{array}{l}\text { Penetrometer } \\
\text { Depth } \\
\text { Markings }\end{array}$} & \multirow{2}{*}{$\begin{array}{l}\begin{array}{l}\text { Before Site } \\
\text { Preparation }\end{array} \\
\mathrm{CH}-3\end{array}$} & \multicolumn{4}{|c|}{ After Site Preparation } \\
\hline & & $\begin{array}{c}\text { Warm } \\
\text { Season (TS, } \\
\text { HP) }\end{array}$ & $\begin{array}{c}\text { Warm } \\
\text { Season } \\
\text { (NTS, HP) }\end{array}$ & $\begin{array}{l}\text { Warm } \\
\text { Season } \\
\text { (TS,S) }\end{array}$ & $\begin{array}{c}\text { Warm } \\
\text { Season } \\
\text { (NTS,S) }\end{array}$ \\
\hline 3" Color: & 0 & 3 & 2 & 3 & 2 \\
\hline 6" Color: & 0 & 0.75 & 0.5 & 1.75 & 0.5 \\
\hline 9" Color: & 0 & 0.25 & 0 & 0.25 & 0 \\
\hline
\end{tabular}

Table 43: CH-3 Compaction Data (Cool Season)

\begin{tabular}{lccccc}
\hline \begin{tabular}{c} 
Penetrometer $\begin{array}{c}\text { Depth } \\
\text { Markings }\end{array}$ \\
\cline { 2 - 6 }
\end{tabular} & $\begin{array}{c}\text { Before Site } \\
\text { Preparation }\end{array}$ & \multicolumn{4}{c}{ After Site Preparation } \\
3" Color: & 0 & $\begin{array}{c}\text { Cool Season } \\
\text { (TS, HP) }\end{array}$ & $\begin{array}{c}\text { Cool Season } \\
\text { (NTS, HP) }\end{array}$ & $\begin{array}{c}\text { Cool Season } \\
\text { (TS,S) }\end{array}$ & $\begin{array}{c}\text { Cool Season } \\
\text { (NTS,S) }\end{array}$ \\
6" Color: & 0 & 2.5 & 1.5 & 2.5 & 2.5 \\
9" Color: & 0 & 1.25 & 0.5 & 0.5 & 0.75 \\
\hline
\end{tabular}

\subsubsection{Objective 4: Examining soil media and amendment products}

\subsubsection{Vegetation Measurement}

The percent cover over a 90 day period was examined for treatments consisting of Topsoil, No Topsoil, Biotic Earth, and ProGanics in Figure 85 and the data can be seen in Appendix 7.5. At 24 days, only No Topsoil and ProGanics surpassed 70\% coverage. At 35 days, all treatments were above $80 \%$ coverage. Long term there was little difference between the treatments. 


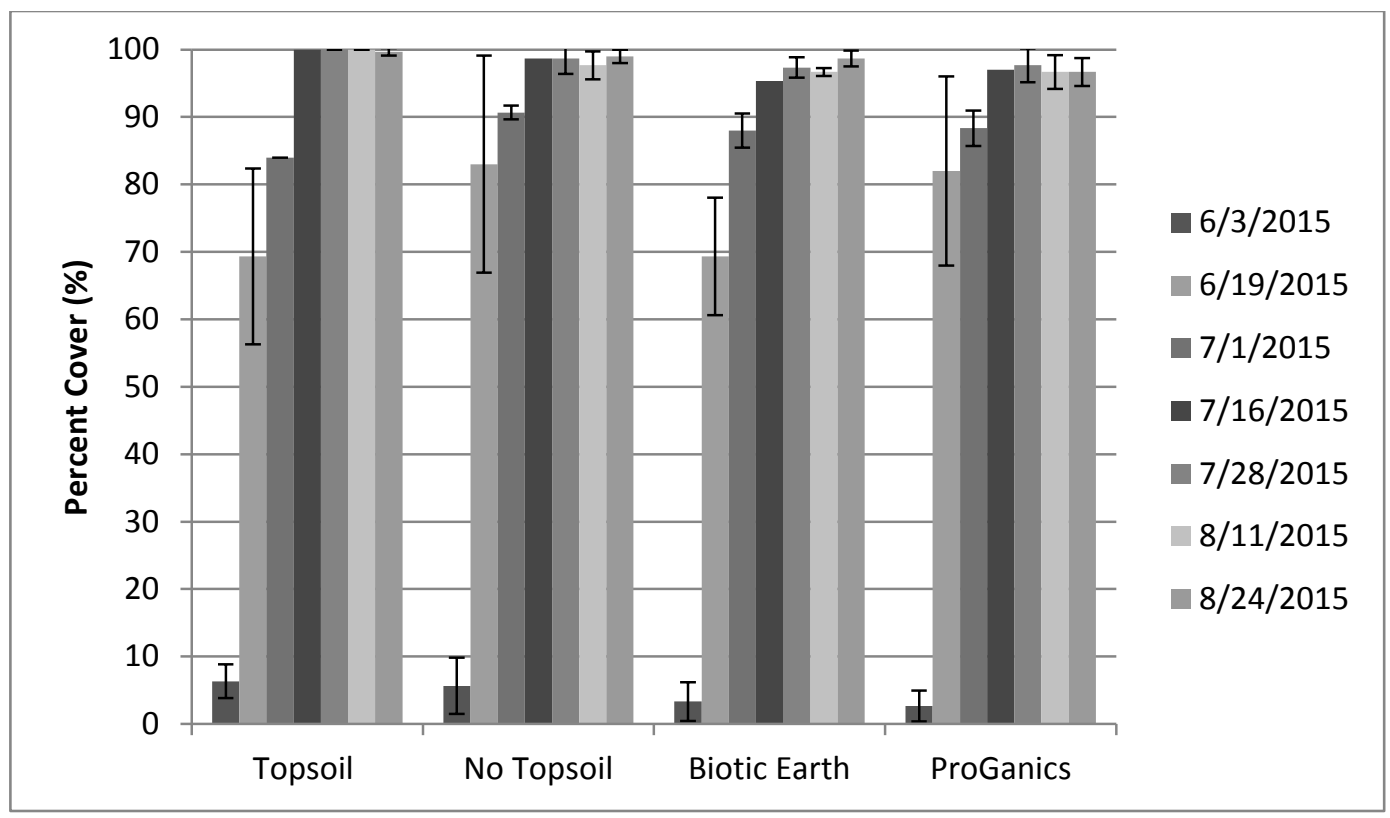

Figure 85: Objective 4 Percent Cover

A percent species comparison was performed 51 days after planting and can be seen in Figure 86 and the data can be seen in Appendix 7.5. From the comparison, Topsoil had $96 \%$ cover by not planted species which was the highest percent out of all the treatments. By cover, Barnyardgrass (Echinochloa crus-galli) was the dominate not planted species in the Topsoil treatment. Again, the high level of not planted species was likely due to the species abundance in the soil from the producer. ProGanics had the lowest percent of no cover and not planted at $5 \%$ and $3 \%$ and as a result had the highest cover by planted species at $92 \%$. 


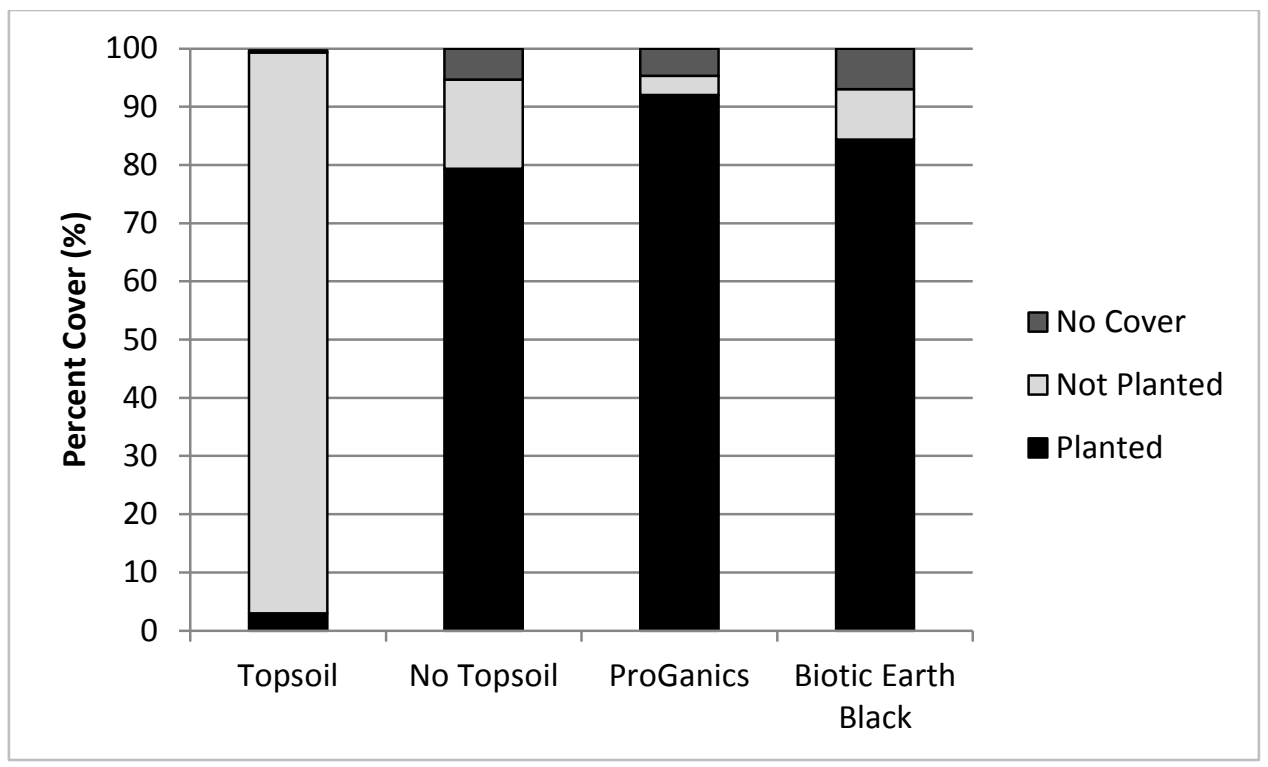

Figure 86: Objective 4 Percent Cover by Species at 51 Days

A percent species comparison at 90 days after planting was performed and can be seen in Figure 87 and the data can be seen in Appendix 7.5. From the comparison, Topsoil had $100 \%$ cover by not planted species which was the highest percent out of all the treatments. The dominate not planted species and reason for coverage was discussed previously. ProGanics had the lowest percent of not planted at $9 \%$ and the highest cover by planted species at $83 \%$.

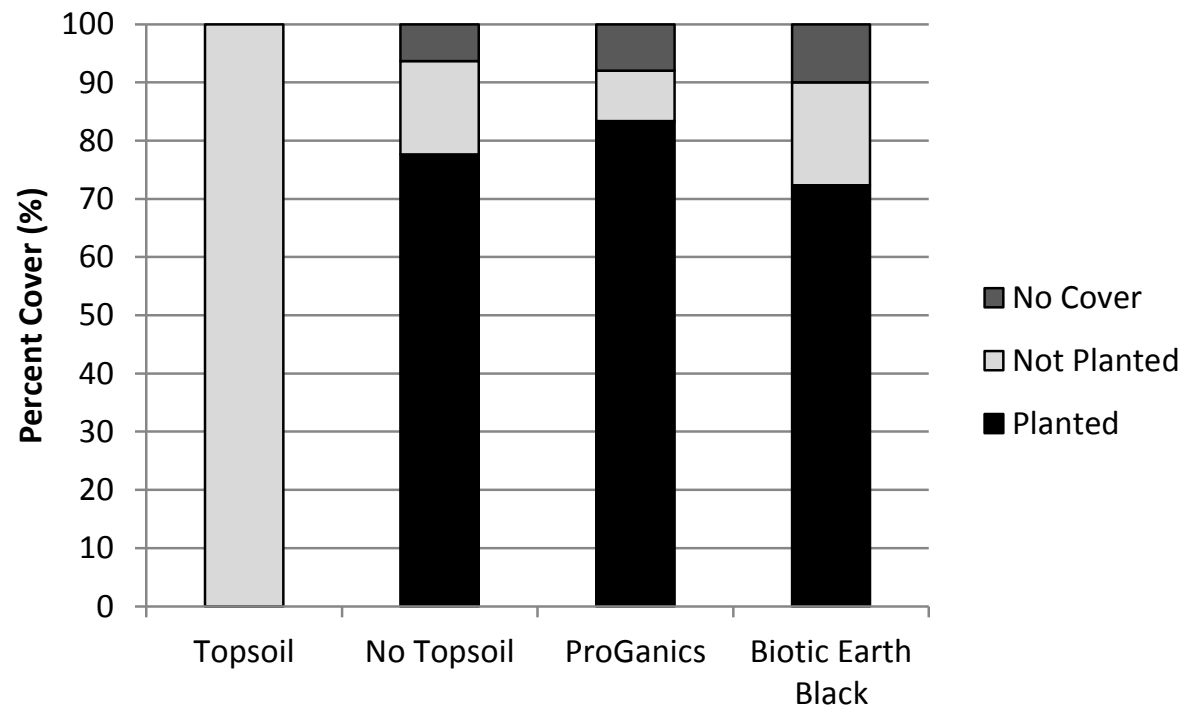

Figure 87: Objective 4 Percent Cover by Species at 90 Days 
A one-way ANOVA analysis on percent cover was conducted to examine four treatments, Biotic Earth, Topsoil, ProGanics, and No Topsoil. The analysis results can be seen in Table 44. The analysis found a p-value of 0.4365 for the four treatments. There was no statistical difference between percent cover for the four treatments. No Topsoil and Topsoil have a higher percent cover compared to Biotic Earth and ProGanics seen in Figure 88. The delayed emergent in the Biotic Earth and ProGanics treatments was likely due to the application method. The products were applied over the seed resulting in a thick cover that would require a longer period of time to grow through the media.

Table 44: Objective 4 Eight Day One-way ANOVA Statistics

\begin{tabular}{lccccc}
\hline Source & DF & $\begin{array}{c}\text { Sum of } \\
\text { Squares }\end{array}$ & $\begin{array}{c}\text { Mean } \\
\text { Square }\end{array}$ & F Ratio & Prob $>$ F \\
\hline Treatment & 3 & 28.33333 & 9.44444 & 1.0119 & 0.4365 \\
Error & 8 & 74.66667 & 9.33333 & & \\
C. Total & 11 & 103.00000 & & & \\
\hline
\end{tabular}

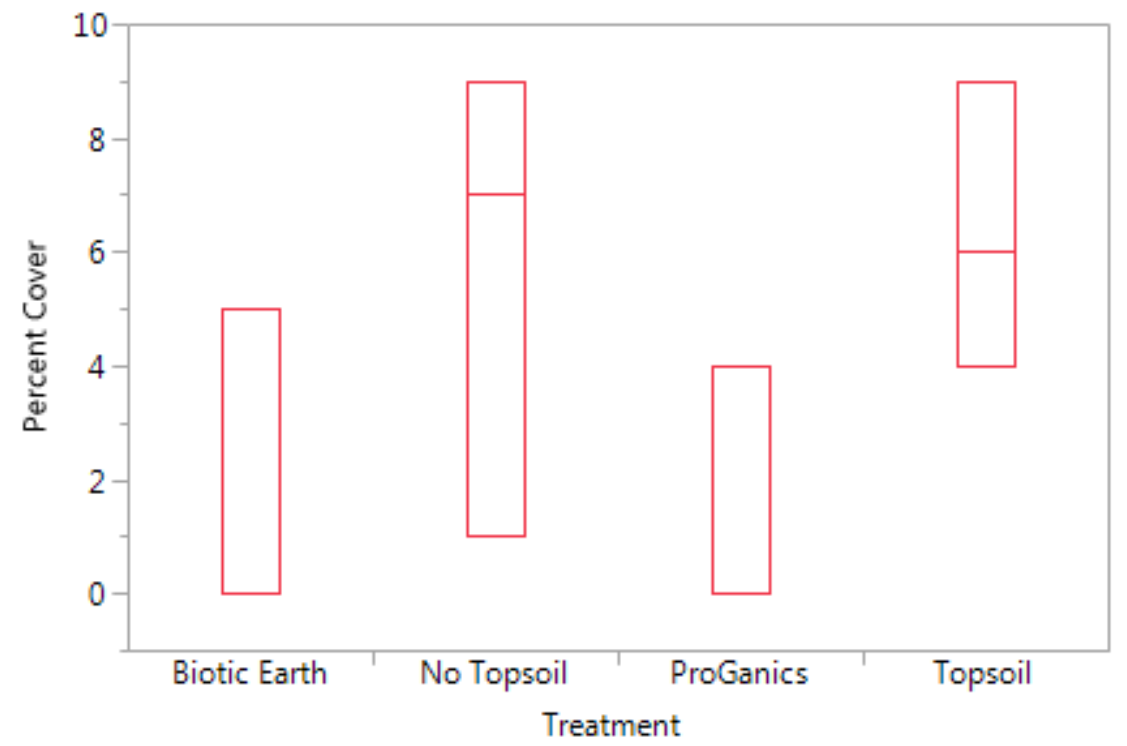

Figure 88: Objective 4 Eight Day One-way ANOVA Graph; $n=3 ; 25 \%-75 \%$ (box); median ( $50 \%$ line in box)

At the end of 90 days, a one-way ANOVA analysis on percent cover was also performed and can be seen in Table 45. The ANOVA found a p-value of 0.9893 indicating no statistical difference between the treatments. Biotic Earth, No Topsoil, ProGanics, and Topsoil all performed similarly with a cover around $90 \%$ seen in Figure 89. 
Table 45: Objective 4 Ninety Day One-way ANOVA Statistics

\begin{tabular}{lccccc}
\hline Source & DF & $\begin{array}{c}\text { Sum of } \\
\text { Squares }\end{array}$ & $\begin{array}{c}\text { Mean } \\
\text { Square }\end{array}$ & F Ratio & Prob $>$ F \\
\hline Treatment & 3 & 131.274 & 43.76 & 0.0399 & 0.9893 \\
Error & 80 & 87815.143 & 1097.69 & & \\
C. Total & 83 & 87946.417 & & & \\
\hline
\end{tabular}

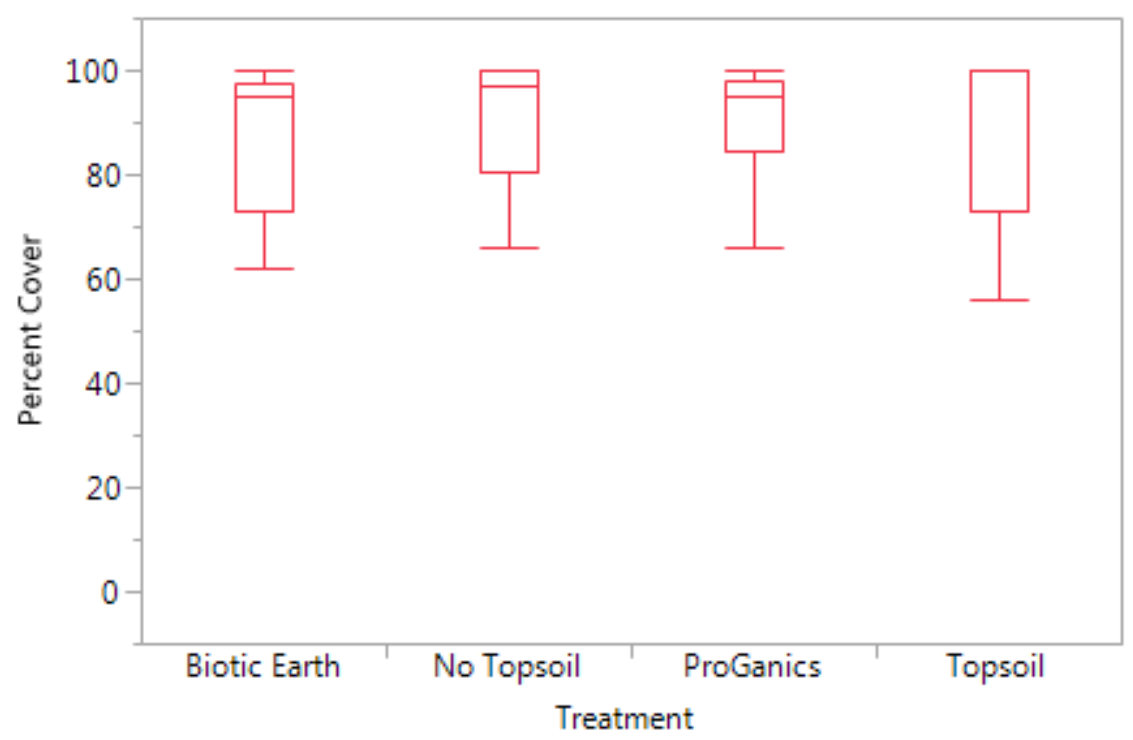

Figure 89: Objective 4 Ninety Day One-way ANOVA Graph; min and max (whisker); 25\%-75\% (box); median

\subsubsection{Monitoring Data} ( $50 \%$ line in box)

The ambient air temperature and precipitation data for this study were the same as in 4.4.1.2 Monitoring Data because the sites were directly beside each other.

Volumetric water content for the various treatments can be seen in Figure 90. At the start of the study, ProGanics had the highest volumetric water content showing a large capability in retaining moisture in the soil. Biotic Earth at the start of the study was below No Topsoil and ProGanics levels, but then towards the middle stayed close to Topsoil and No Topsoil levels.

When precipitation decreased and dryer conditions were present, the Biotic Earth and ProGanics had low volumetric water content. The two products could have been wicking the moisture towards the surface out of the range of the soil sensor located at a depth $0.5 \mathrm{ft}(0.15$ $\mathrm{m}$ ) while retaining the moisture at the soil surface. The two treatments could have also been 
wicking the moisture towards the surface into the products and then evaporating of the products. A more intensive examination could answer the cause behind the phenomenon.

During dryer conditions, Topsoil and No Topsoil did not experience dramatic jumps in volumetric water content like the other two treatments. Overall, Topsoil and No Topsoil treatments were similar in nature except when Topsoil dropped almost $10 \%$ in the middle of June compared to No Topsoil numbers.

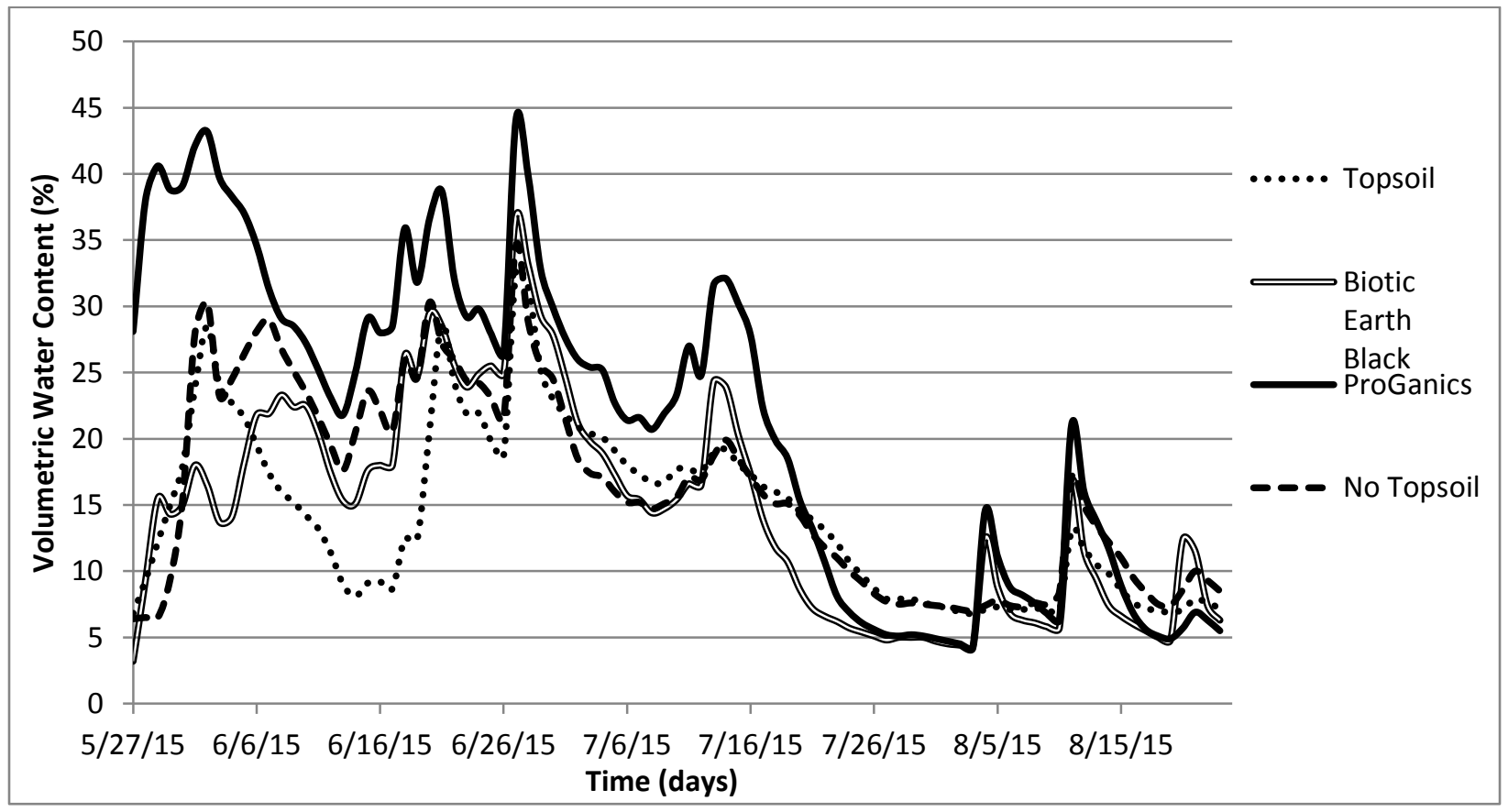

Figure 90: Objective 4 Mean Volumetric Water Content

Again, the initial site compaction data in Table 46 shows severe compaction conditions for the top 9 in. After site preparation, the first 3 in. for the topsoil subplots improved to a rank of "Green" where no topsoil and Biotic Earth subplots only improved to "Red". ProGanics however compaction improved to a rank of "Yellow". At 6 in., topsoil and no topsoil improved to a rank of "Red" where ProGanics and Biotic Earth remained at "Max". All treatments remained at a rank of "Max" at 9 inches. The compaction ranking and classification can be seen in Table 28. An explanation of rank and scale can be seen in Section 4.4.1.2. 
Table 46: CH-1B Compaction Data

\begin{tabular}{lccccc}
\hline $\begin{array}{c}\text { Penetrometer } \\
\text { Depth } \\
\text { Markings }\end{array}$ & $\begin{array}{c}\text { Before Site } \\
\text { Preparation }\end{array}$ & \multicolumn{4}{c}{ After Site Preparation } \\
\cline { 2 - 6 } 3" Color: & CH-1B & Topsoil & No Topsoil & ProGanics & Biotic Earth \\
6" Color: & 0 & 3.0 & 0.8 & 1.7 & 1.0 \\
9" Color: & 0 & 0.7 & 0.7 & 0.5 & 0.5 \\
\hline
\end{tabular}

\subsection{Discussion}

The study found for Objective 1 that the developed seed mixtures performed as well or better than the current WVDOH seed mixtures. Native and low invasive mixtures could be utilized for roadside construction reclamation in West Virginia. Over time, the native mixtures would provide an ecological benefit to the state by increasing native diversity and reducing the planting of non-native species. The native mixtures incorporate various flowering species that provide beautification to roadsides and nectar sources for pollinating animal species.

The non-native mixtures reduce the introduction and spread of invasive species compared to current practices. The mixtures have proven to provide adequate vegetative cover to protect soils from erosion while being low growing that reduces the rate of mowing. Reduced mowing occurrence saves valuable tax dollars and decreases soil disturbance from low cutting mowers and equipment tracks. Again, the largest benefit to these mixtures was the elimination of all threat level 1 and 2 species in an effort to control and reduce the spread of highly invasive species that cause ecological damage.

For Objective 2, a specific high elevation seed mixture was found to be not necessary. Cool Season and Mowable Areas mixtures performed well and they achieved $70 \%$ vegetative cover. The native high elevation specific mixture did not perform well and never reached the $70 \%$ cover margin. If a native mixture is required or desired, further research and development would be required. The colder temperatures associated with high elevations do not fare well to a warm season native mixture. 
A cool season native mixture could be a viable option seeing that the non-native equivalent performed well under the high elevation conditions. One important factor that would need to go into a cool season native mixture would be a forb or legume species. The non-native mixtures were predominantly covered by legumes that provided cover to the slower developing graminoids species. If a native forb or legume could perform similarly, the graminoids would likely develop in the same manor increasing the overall percent cover.

Seed bed preparation for Objective 3 did not show any statistical significance long term between treatments. However, percent cover by species did show that treatments with topsoil did have a higher percent of non-planted species than non-topsoil treatments. Quality of topsoil should be a concern to control the potential spread of undesirable species that may or may not over run a reclamation project.

Objective 1 and 3 testing demonstrated that successful vegetation cover and performance was achieved at a low and medium elevation in West Virginia. WVDOH Medians, Warm Season, and Cool Season were tested at these two varying elevations. The mixtures all showed performances that reach and exceeded $70 \%$ cover in only one growing season. Seed mixtures that perform well at varying elevations in West Virginia was important to reduce the number of seeding options available in the WVDOH specifications.

In Objective 4, no long term difference in the first growing season was discovered between the treatments. Application method likely slowed the initial vegetation emergent rate of Biotic Earth and ProGanics, but at 90 days there was no difference present. Again, the topsoil used did show high levels of introduced species indicating proper topsoil management and quality should be of high consideration to ensure desired reclamation results.

Overall, native and low invasive seed mixtures could be used for reclamation purposes along highway corridors. These native and low invasive mixtures performed well at a medium and low elevation in West Virginia. Only the low invasive seed mixtures performed well at a high elevation in the state. Proper seedbed preparation practices did not affect the percent cover, but 
did indicate the importance of topsoil quality and management. Soil media and amendment products could reduce or replace the need for topsoil, but under moderate soil conditions did not show significant difference then subsoil conditions.

\subsection{Conclusion}

The purpose of this chapter was to test and examine the main concerns of the West Virginia Division of Highways pertaining to highway construction reclamation. The concerns focused on performance of alternative seed mixtures, vegetation cover at high elevations, and seedbed preparation influences on vegetation cover in order to obtain a minimum of $70 \%$ vegetative cover to meet NPDES permitting. As a result, the following objectives were developed and tested to answer these concerns.

1. Compare the proposed seed mixtures to the current standard.

2. Evaluate the need for a high elevation seed mixture.

3. Determine if seed bed preparation influences proposed seed germination and cover.

4. Evaluate the performance of soil amendments in comparison to topsoil and no topsoil. Through testing, the study was able to answer the four objectives mentioned above. The study found that alternative seed mixtures performed as well or better than the current standard mixtures and a high elevation specific mixture was not required. Through proper seed bed preparation techniques, no influence on seed germination or cover was determined. Finally, soil media and amendment products did not positively influence vegetative growth on a site with moderate soil conditions compared to using topsoil and subsoil. 


\subsection{Conclusions and Recommendations}

Through the development of seed mixtures specific to highway reclamation in West Virginia and addressing and testing the main concerns of the WVDOH, definitive conclusions could be drawn. The Developed Type A-D seed mixtures were tested in the first objective to compare them to the current WVDOH Type B and D seed mixtures. The second objective evaluated the need of a high elevation specific mixture, Developed Type D1, in comparison to Developed Type A-C and WVDOH Type B seed mixtures at an elevation of 3,294 ft. Objective 3 studied seed bed preparation influences on proposed seed germination and cover for Developed Type B and C and WVDOH Type B seed mixtures. Finally, the fourth objective evaluated the performance of soil amendments in comparison to topsoil and no topsoil subplots.

Developed seed mixtures Type A-D1 were all tested in the field. Developed Type A-C all performed equally or better than the current WVDOH Type B and D proving they are viable alternatives. Developed Type D1 performed equally to the WVDOH Type B and D at a low elevation, but was unable to perform to the same quality at a high elevation compared to WVDOH Type B. Developed Type D1 would not be recommended as an alternative seed mixture to the WVDOH due to poor performance. Developed Type D2 and E were not tested in the field due to the lack of site conditions to test them on. Therefore, no recommendation for those mixtures could be made.

A high elevation specific seed mixture would not be required because field testing performance indicated that non-native Developed Type A and C surpassed the $70 \%$ cover margin while the native high elevation specific mixture, Developed Type D1, was unable to break $70 \%$ vegetative cover. If a native mixture was desired to perform well at high elevations, further research and development would be required. The tested Developed Type D1 was a warm season native mixture that displayed poor coverage under cooler high elevation 
conditions. A cool season native mixture could potentially overcome the colder conditions at high elevations, but would need to be developed, tested, and confirmed.

By testing various proper seedbed preparation techniques, no influence on seed germination or cover was detected. However, there was no comparison between proper and improper seedbed preparation techniques. Construction practices do not always follow industry proven techniques and as a result could be a cause behind failed vegetation establishment. Future testing could examine common construction practices in West Virginia to proper practices to verify the magnitude of variance.

Soil media and amendment products were compared to topsoil and subsoil conditions to examine any difference in vegetative cover. No long term influence was observed between Biotic Earth, ProGanics, topsoil, and subsoil treatments. The lack of change could be due to the moderate soil conditions that were previously vegetated. The same or more intensive examination should be conducted on a new construction site with poor soil quality. The test should also be repeated in large scale to confirm results.

Large scale testing should also be conducted to examine the performance and repeatability of the seed mixtures at varying elevations in West Virginia. The performance and repeatability would indicate any undesirable traits and the dependability of the seed mixtures. The final recommendation would be a large scale examination of various hydraulic erosion control products at various slope inclinations to test erosion control efficiencies and abilities in West Virginia. Overall, the study was a success in addressing the main concerns of the West Virginia Division of Highways to improve vegetative performance along roadways in West Virginia. Further research in the addressed topics above would positively influence the understanding of the various variables that affect vegetative cover and erosion control in West Virginia. 


\subsection{References}

Anderson, M.K. (2003). "Plant Guide for wild bergamot (Monarda fistulosa)." USDA-NRCS, National Plant Data Center c/o Department of Plant Sciences, University of California, Davis, CA. <http://plants.usda.gov/plantguide/pdf/cs_mofi.pdf>. (January 14, 2015).

Bakr, N., Weindorf, D.C., Zhu, Y., Arceneaux, A.E., and Selim, H.M. (2012). "Evaluation of compost/mulch as highway embankment erosion control in Louisiana at the plot-scale." Journal of Hydrology, 468-469, 257-267.

Benik, S.R., Wilson, B.N., Biesboer, D.D., Hansen, B., and Stenlund, D. (2003). "Evaluation of erosion control products using natural rainfall events." Journal of Soil and Water Conservation, 58(2), 98-105.

Bhattacharyya, R., Smets, T., Fullen, M.A., Poesen, J., and Booth, C.A. (2010). "Effectiveness of geotextiles in reducing runoff and soil loss: A synthesis." Catena, 81, 184-195.

Bochet, E, Tormo, J., and Garcia-Fayos, P. (2010). "Native Species for Roadslope Revegetation: Selection, Validation, and Cost Effectiveness." Restoration Ecology, 18(5), 656-663.

Bureau of Land Management, Farmington Field Office (2013). "Reduced Palatability Seed Mix." Bare Soil Reclamation Procedures (Appendix A- Surface Use Plan of Operations)(Appendix B- Rights of Way Plan of Operations), <http://www.blm.gov/pgdata/etc/medialib/blm/nm/field_offices/farmington/farmington_pla nning/updated_reclamation2.Par.31806.File.pdf/NEW_Reduced_Palatability_Seed_Mix_ 1-15-13.pdf>, (6/4/2014).

Bush, T. (2002). "Plant Fact Sheet for birdsfoot trefoil (Lotus corniculatus)." USDA-NRCS, Rose Lake Plant Materials Center, East Lansing, MI. $<$ http://plants.usda.gov/factsheet/pdf/fs_loco6.pdf>. (November 18, 2014).

California Department of Transportation (2014). "Specifying seed and plant species." Erosion Control Toolbox, <http://www.dot.ca.gov/hq/LandArch/ec/plants/seed.htm>. (June 2, 2014).

Crowder, W. (N.D.)"Plant Fact Sheet for hard fescue (Festuca brevipila)." USDA-NRCS, Plant Materials Center, Pullman, WA. <http://plants.usda.gov/factsheet/pdf/fs_febr7.pdf>. (November 13, 2014).

Casey, P.A. (2012). "Plant Guide for cereal rye (Secale cereale)." USDA-NRCS, Plant Materials Center, Elsberry, MO, <http://plants.usda.gov/plantguide/pdf/pg_sece.pdf>. (February 12, 2015).

Calloudon, B., Eshelman, K., Gianola, J., Habich, N., Hughes, L., Johnson, C., Pellant, M., Podborny, P., Rasmussen, A., Robles, B., Shaver, P., Spehar, J., Willoughby, J., (1996). "Sampling vegetation attributes." Technical Reference 1734-4, Denver, Colo.: US Department of Agriculture. <http://www.blm.gov/nstc/library/pdf/samplveg.pdf>. (August 5, 2015). 
Dickerson, J., Wark, B., Poole, B., Burgdorf, D., Maher, R., Bush, T., and Miller, C. (1998). "Vegetating with Native Grasses in Northeastern North America." B. Poole, eds., USDANRCS and Ducks Unlimited Canada, 1998.

Dunifon, S.N., Evanylo, G.K., Maguire, R.O., and Goatley, J.M. Jr. (2011). "Soil nutrient and fescue (Festuca app.) responses to compost and hydroseed on a disturbed roadside." Compost Science \& Utilization, 19(3), 147-151.

Elzinga, C. L., Salzer, D. W., Willoughby, J. W., (1998). "Measuring and monitoring: Plant population." Technical Reference 1730-1, Denver, Colo.: US Bureau of Land Management. <http://www.blm.gov/nstc/library/pdf/MeasAndMon.pdf>. (August, 8 2015).

Ernst Conservation Seeds (2014). Ernst Conservation Seeds Catalog. $<$ http://www.ernstseed.com/files/catalog/ernst-seeds-catalog.pdf>, (December, 14, 2014).

Faucette, L.B., Risse, L.M., Jordan, C.F., Cabrera, M.L., Coleman, D.C., and West, L.T. (2006). "Vegetation and soil quality effect from hydroseed and compost blankets used for erosion control in construction activities." Journal of Soil and Water Conservation Society, 61(6), 355-362.

Hansen, N.E., Vietor, D.M., Munster, C.L., White, R.H., and Provin, T.L. (2012). "Runoff and nutrient loss from constructed soils amended with compost." Applied and Environmental Soil Science, 2012, 542873., doi:10.1155/2012/542873.

Hargett, D.L., Phillips, J.A., and Kleiss, H.J. (1982). "Soil variability and fertility considerations affecting establishment of erosion control vegetation on Piedmont roadcuts." Journal of Soil and Water Conservation Society, 37(4), 229-233.

Hogan, M. and Drake, K. (2009a). "Guiding Principle 11: Protect or Optimize Appropriate Vegetation Community." Sediment Source Control Handbook: An Adaptive Approach to Restoration of Disturbed Areas, K. Drake, B. Moss, and K. Gilbert, eds. Sierra Business Council, Truckee, CA, 41.

Hogan, M. and Drake, K. (2009b). "Tool 11 - Vegetative Treatments." Sediment Source Control Handbook: An Adaptive Approach to Restoration of Disturbed Areas, K. Drake, B. Moss, and K. Gilbert, eds. Sierra Business Council, Truckee, CA, 130-136.

Houck, M. J. (2009). "Plant materials technical note No. 12: Calculating seed mixtures - Steps to guide you in developing conservation seed mixtures." Technical Notes, Natural Resources Conservation Service, Alexandria, Louisiana.

Houck, M.J. and Row, J.M. (2006). "Plant Guide for partridge pea (Chamaecrista fasciculate)." USDA-NRCS. <http://plants.usda.gov/plantguide/pdf/pg_chfa2.pdf>. (January 30, 2015).

Jimmy Carter Plant Materials Center. (2011). "Plant Fact Sheet for switchgrass (Panicum virgatum L.)." USDA-NRCS, < http://plants.usda.gov/factsheet/pdf/fs_pavi2.pdf>. (November 11, 2014). 
Kirk, S. and Belt, S. (2009). "Plant Fact Sheet for panicledleaf ticktrefoil (Desmodium paniculatum)." USDA-Natural Resources Conservation Service, Norman A. Berg National Plant Materials Center, Beltsville, 20705.

<http://plants.usda.gov/factsheet/pdf/fs_depa6.pdf>. (November 11, 2014).

Kirk, S. and S.Belt. (2010). "Plant Fact Sheet for blue vervain (Verbena hastata)." USDA-Natural Resources Conservation Service, Norman A. Berg National Plant Materials Center, Beltsville, MD 20705. <http://plants.usda.gov/factsheet/pdf/fs_veha2.pdf>. (November 20, 2014).

Kirmer, A., Baasch, A., and Tischew, S. (2012). "Sowing of low and high diversity seed mixtures in ecological restoration of surface mined-land." Applied Vegetation Science, 15, 198207.

Landis, T.D., Wilkinson, K.M., Steinfeld, D.E., Riley, S.A., and Fekaris, G.N. (2005). "Roadside revegetation of forest highways: new applications for native plants." Native Plants Journal, 6(3), 297-305.

Li, M., Schutt, J.R., McFalls, J., Bardenhagen, E.K., Sung, C.Y., and Wheelock, L. (2008). "Successional establishment, mowing response, and erosion control characteristics of roadside vegetation in Texas." Texas Department of Transportation. FHWA/TX-08/04949-1.

Lyman, O.R., and Longnecker, M. (2001). "An introduction to statistical methods and data analysis." Chapter 15 Analysis of Variance for Standard Designs, Duxbury, Pacific Grove, CA, 855-859.

MacDonagh, P. and Hallyn, N. (2010). "Native seed mix design for roadsides." Minnesota Department of Transportation. MN/RC 2010-20.

Mallik, A.U. and Karim, M.N. (2008). "Roadside revegetation with native plants: Experimental seeding and transplanting of stem cuttings." Applied Vegetation Science, 11, 547-554.

Oakley, C.A. and Knox, J.S. (2013). "Plant species richness increases resistance to invasion by non-resident plant species during grassland restoration." Applied Vegetation Science, 16, 21-28.

Owsley, M. (2011a). "Plant Fact Sheet for big bluestem (Andropogon gerardii Vitman)." USDANatural Resources Conservation Service, Jimmy Carter PMC Americus, Georgia 31719. $<$ http://plants.usda.gov/factsheet/pdf/fs_ange.pdf>. (February 12, 2015).

Owsley, M. (2011b). "Plant Fact Sheet for indiangrass (Sorghastrum nutans[ L.] Nash)." USDANRCS, Jimmy Carter PMC Americus, Georgia 31719. <http://plants.usda.gov/factsheet/pdf/fs_sonu2.pdf>. (November 11, 2014).

Pidwirny, M. (2006). "Plant Succession". Fundamentals of Physical Geography, 2nd Edition. <http://www.physicalgeography.net/fundamentals/9i.html>. (July 23, 2014). 
Profile Products LLC. (2015a). "ProMatrix Engineered Fiber Matrix (EFM)." $<$ http://www.profileevs.com/productpage/promatrix-engineered-fiber-matrix-efm>. (June 17, 2015).

Profile Products LLC. (2015b). "Tornado Tack ${ }^{T M}$ ST-1000." < file:///E:/RP-293/RP293\%20Project\%20Documents/A012-32594_Tornado\%20Tack\%20Sell\%20Sheet.pdf>. (June 30, 2015).

Profile Products LLC. (2015c). "Application Guide for ProGanics ${ }^{\mathrm{TM}}$ Biotic Soil Media ${ }^{\mathrm{TM}}\left(\mathrm{BSM}^{\mathrm{TM}}\right)$." <http://profilelibrary.info/Files/ProGanics\%20BSM\%20App\%20Guide_5-21-15.pdf>. (June 30, 2015).

Profile Products LLC. (2015d). "ProGanics Info Sheet." $<$ http://profilelibrary.info/Files/ProGanics\%20Info\%20Sheet_full\%20doc.pdf>. (June 30, 2015).

USDA-NRCS (2002a). "Plant Fact Sheet for deertongue (Dichanthelium clandestinum)." Plant Materials Program. <http://plants.usda.gov/factsheet/pdf/fs_dicl.pdf >. (November 11, 2014).

USDA-NRCS (2002b). "Plant Fact Sheet for little bluestem (Schizachyrium scoparium)." Plant Materials Program. National Plant Materials Center, Beltsville, MD. < http://plants.usda.gov/factsheet/pdf/fs_scsc.pdf >. (November 11, 2014).

USDA-NRCS (2002c). "Plant Fact Sheet for black-eyed susan (Rudbeckia hirta)." Plant Materials Program. <http://plants.usda.gov/factsheet/pdf/fs_ruhi2.pdf>. (November 11, 2014).

USDA-NRCS (2002d). "Plant Fact Sheet for redtop (Agrostis gigantea)." Plant Materials Program. < http://plants.usda.gov/factsheet/pdf/fs_aggi2.pdf>. (November 13, 2014).

USDA-NRCS (2002e). "Plant Fact Sheet for red fescue (Festuca rubra)." Plant Materials Program. < http://plants.usda.gov/factsheet/pdf/fs_feru2.pdf>. (November 18, 2014).

USDA-NRCS (2002f). "Plant Fact Sheet for white clover (Trifolium repens)." Plant Materials Program. < http://plants.usda.gov/factsheet/pdf/fs_trre3.pdf>. (November 18, 2014).

USDA-NRCS (2002g). "Plant Fact Sheet for weeping lovegrass (Eragrostis curvula)." Plant Materials Program. < http://plants.usda.gov/factsheet/pdf/fs_ercu2.pdf>. (February 11, 2014).

USDA-NRCS (2002h). "Plant Fact Sheet for reed canarygrass (Phalaris arundinacea)." Plant Materials Program. < http://plants.usda.gov/factsheet/pdf/fs_phar3.pdf>. (February 11, 2014).

USDA, NRCS (2015). "The PLANTS Database." National Plant Data Team, Greensboro, NC 27401-4901 USA. <http://plants.usda.gov>. (February 10, 2015). 
U.S. Environmental Protection Agency (2013). "Level III ecoregions of the continental United States." U.S. EPA National Health and Environmental Effects Research Laboratory, Corvallis, Oregon, <ftp://ttp.epa.gov/wed/ecoregions/us/Eco_Level_III_US.pdf>. (August 12, 2014).

Rentch, J.S., Fortney, R.H., Stephenson, S.L., Adams, H.S., Grafton, W.N., and Anderson, J.T. (2005). "Vegetation-site relationships of roadside plant communities in West Virginia, USA." Journal of Applied Ecology, 42, 129-138.

Reubens, B., Poesen, J., Danjon, F., Geudens, G., and Muys, B. (2007). "The role of fine and coarse roots in shallow slope stability and soil erosion control with a focus on root system architecture: a review." Trees, 21, 385-402.

Roberts, J. (2001). "Choosing Seed for the Lawn." University of New Hampshire Cooperative Extension, <http://www.ceinfo.unh.edu>. (June 2, 2014).

Robichaud, P.R., Jordan, P., Lewis, S.A., Ashmun, L.E., Covert, S.A., and Brown, R.E. (2013). "Evaluating the effectiveness of wood shred and agricultural straw mulches as a treatment to reduce post-wildfire hillslope erosion in southern British Columbia, Canada." Geomorphology, 197, 21-33.

Royal Botanic Gardens, Kew. (2015). Seed Information Database (SID). $<$ http://data.kew.org/sid/>. (August 5, 2015)

Salon, P.R. and Miller, C.F. (2012) "A Guide to: Conservation Plantings on Critical Areas for the Northeast." USDA, NRCS, Big Flats Plant Materials Center, Corning, NY, <http://plantmaterials.nrcs.usda.gov/nypmc/>. (June 10, 2014).

Sanderson, M. A., Brink, G., Ruth, L., and Stout, R. (2012). "Grass-legume mixtures suppress weeds during establishment better than monocultures." Agronomy Journal, 104 (1), 3642.

Shadow, R.A. (2009). "Plant Fact Sheet for virginia wildrye (Elymus virginicus)." USDA-NRCS, East Texas Plant Materials Center. <http://plants.usda.gov/plantguide/pdf/pg_elvi3.pdf>. (February 12, 2015).

Sheahan, C.M. (2012a). "Plant Fact Sheet for narrowleaf mountainmint (Pycnanthemum tenuifolium). USDA-NRCS, Cape May Plant Materials Center. Cape May, NJ. 08210. $<$ http://plants.usda.gov/factsheet/pdf/fs_pyte.pdf >. (January 14, 2015).

Sheahan, C.M. 2012b. "Plant Fact Sheet for flat-top goldentop (Euthamia graminifolia)." USDANRCS, Cape May Plant Materials Center,Cape May, NJ. 08210. $<$ http://plants.usda.gov/factsheet/pdf/fs_eugr5.pdf>. (January 20, 2014).

Skousen, J.G. and Venable, C.L. (2007). "Establishing native plants on newly-constructed and older-reclaimed sites along West Virginia Highways." Land Degradation \& Development, 19, 388-396. 
Skrindo, A.B. and Halvorsen, R. (2008). "Natural revegetation on forest topsoil and subsoil along roadsides in boreal forest." Applied Vegetation Science, 11(4), 483-490.

Smets, T., Poesen, J., and Knapen, A. (2008). "Spatial scale effects on the effectiveness of organic mulches in reducing soil erosion by water." Earth-Science Reviews, 89, 1-12.

Steinfeld, D.E., Riley, S.A., Wilkinson, K.M., Landis, T.D., and Riley, L.E. (2007a). "Ch. 6Planning Phase Three: Vegetation Analysis." Roadside revegetation: An integrated approach to establishing native plants. U.S. Forest Service. FHWA-WFL/TD-07-005.

Steinfeld, D.E., Riley, S.A., Wilkinson, K.M., Landis, T.D., and Riley, L.E. (2007b). "10.3.1Seeding." Roadside revegetation: An integrated approach to establishing native plants. U.S. Forest Service. FHWA-WFL/TD-07-005.

Stevens, M. (2003). "Plant Fact Sheet for soft rush (Juncus effusus)." USDA-NRCS, Formerly USDA-NRCS National Plant Data Center. $<$ http://plants.usda.gov/plantguide/pdf/cs_juef.pdf>. (February 12, 2015).

Storey, B.J., Schutt, J.R., McFalls, J.A., Jones, K.D., Garza, A.P., Gaus, T.A., Marek, G., Heflin, K., Rogers, W.J., and Robinson,C. (2011). "Synthesis and study of the roadside vegetation establishment process." Texas Department of Transportation. FHWA/TX11/0-5731-1.

Taliga, C.E., Wynia, R.L., Belt, S.V. (2012). "Plant Fact Sheet for false sunflower (Heliopsis helianthoides (L.) Sweet)." USDA-Natural Resources Conservation Service, Denver State Office. Denver CO., 80225-0426.

$<$ http://plants.usda.gov/factsheet/pdf/fs_hehe5.pdf>. (November 11, 2014).

Tyser, R.W., Asebrook, J.M., Potter, R.W., and Kurth, L.L. (1998). "Roadside revegetation in Glacier National Park, U.S.A.: Effects of herbicide and seeding treatments." Restoration Ecology, 6(2), 197-206.

Verdyol (N.D.). "Biotic Earth ${ }^{\mathrm{TM}}$ Biotic Soil Amendment (BSA) Installation \& Mixing Guide." $<$ http://www.erosioncontrolblanket.com/wpcontent/uploads/2015/07/installation_mixing_guide-BSA-2015.pdf>. (April 30, 2015).

Verdyol (2015a). "Biotic Earth ${ }^{\mathrm{TM}}$ Black."

$<$ http://www.brockwhite.com/files/resource/other/hgm_biotic_earth_black_SPEC_SHEET .pdf>. (July 2, 2015).

Verdyol (2015b). "Biotic Earth ${ }^{\mathrm{TM}}$ Hydraulic Growth Mediums (HGM) \& Earth Bound® Soil Stabilizer \& Tack Application Rates." <http://www.brockwhite.com/files/resource/other/BIOTIC_EARTH_BLACK_application_r ates.pdf>. (July 2, 2015). 
Wennerberg, S. (2006). "Plant Fact Sheet for fox sedge (Carex vulpinoidea)." Formerly USDANRCS National Plant Data Center, Baton Rouge, LA, $<$ http://plants.usda.gov/plantguide/pdf/pg_cavu2.pdf>. (February 12, 2015).

West Virginia Department of Environmental Protection (WVDEP). 2006. "Section 3.11Permanent Seeding" Erosion and Sediment Control Best Management Practice Manual: Charleston, West Virginia <http://www.bluefieldstormwater.org/uploads/3/0/1/1/3011957/erosion_sediment_contr ol_manual.pdf >. (December 2014).

West Virginia Department of Highways (WVDOH) (2010). "Section 652-Seeding and Mulching" Standard Specifications Roads and Bridges: Charleston, West Virginia <http://www.transportation.wv.gov/highways/contractadmin/specifications/Documents/20 10\%20Standard\%20Specifications\%20Roads\%20and\%20Bridges/Complete\%20Publica tions/2010StandardRoadsnBridges.pdf>. (November 2014).

West Virginia Division of Natural Resources (WVDNR) Natural Heritage Program (2009). Invasive Plant Species of West Virginia.

$<$ http://www.wvdnr.gov/Wildlife/Handout\%20Invasive\%20Plants\%20of\%20WV\%202009. pdf>. (February 11, 2015). 


\subsection{Appendix}

\subsection{Planting and Examination Schedule}

\begin{tabular}{|c|c|c|c|c|c|c|c|c|c|}
\hline $\begin{array}{l}\text { Plant } \\
\text { Date }\end{array}$ & $\begin{array}{l}\text { Product }^{1} \\
\text { Application }\end{array}$ & Examine & Examine & $\begin{array}{c}\text { Apply }^{2} \\
\text { Fertilizer }\end{array}$ & Examine & Examine & Examine & Examine & Examine $^{3}$ \\
\hline $\begin{array}{c}\text { May 27, } \\
2015\end{array}$ & $\begin{array}{c}\text { May, } 28 \\
2015\end{array}$ & $\begin{array}{c}\text { June 3, } \\
2015\end{array}$ & $\begin{array}{c}\text { June 19, } \\
2015\end{array}$ & $\begin{array}{c}\text { June 24, } \\
2015\end{array}$ & $\begin{array}{c}\text { July 1, } \\
2015\end{array}$ & $\begin{array}{c}\text { July 16, } \\
2015\end{array}$ & $\begin{array}{c}\text { July 28, } \\
2015\end{array}$ & $\begin{array}{c}\text { Aug. 11, } \\
2015\end{array}$ & $\begin{array}{c}\text { Aug. 24, } \\
2015\end{array}$ \\
\hline
\end{tabular}




\subsection{Percent Cover Data for Objective 1}

Table 47: Objective 1 Percent Cover Over 90 Days ( $n=3$ for each treatment)

\begin{tabular}{|c|c|c|c|c|c|c|c|}
\hline $\begin{array}{l}\text { Data } \\
\text { Collection } \\
\text { Date }\end{array}$ & $6 / 3 / 15$ & $6 / 19 / 15$ & $7 / 1 / 15$ & $7 / 16 / 15$ & $7 / 28 / 15$ & $8 / 11 / 15$ & $8 / 24 / 15$ \\
\hline & \multicolumn{7}{|c|}{ Mean Percent Cover } \\
\hline $\begin{array}{l}\text { DOH } \\
\text { Medians }\end{array}$ & 12.7 & 86.0 & 98.0 & 100.0 & 99.7 & 100.0 & 100.0 \\
\hline $\begin{array}{l}\text { DOH } \\
\text { Cut/Fill }\end{array}$ & 7.7 & 68.0 & 91.7 & 99.7 & 100.0 & 100.0 & 100.0 \\
\hline $\begin{array}{l}\text { Mowable } \\
\text { Areas }\end{array}$ & 15.7 & 93.0 & 98.7 & 100.0 & 100.0 & 100.0 & 100.0 \\
\hline $\begin{array}{l}\text { Warm } \\
\text { Season }\end{array}$ & 0.3 & 51.7 & 88.7 & 99.7 & 100.0 & 100.0 & 100.0 \\
\hline $\begin{array}{l}\text { Cool } \\
\text { Season }\end{array}$ & 9.7 & 70.3 & 94.3 & 100.0 & 99.7 & 100.0 & 99.0 \\
\hline $\begin{array}{l}\text { High } \\
\text { Elevation }\end{array}$ & 4.0 & 74.3 & 94.3 & 100.0 & 100.0 & 100.0 & 100.0 \\
\hline
\end{tabular}

Table 48: Objective 1 Percent by Species Data at 51 Days ( $n=3$ for each treatment)

\begin{tabular}{lccc}
\hline & $\begin{array}{c}\text { Mean } \\
\text { Planted }\end{array}$ & $\begin{array}{c}\text { Mean Not } \\
\text { Planted }\end{array}$ & $\begin{array}{c}\text { Mean No } \\
\text { Cover }\end{array}$ \\
\hline DOH Medians & 83 & 17 & 0 \\
DOH Cut/Fill & 82 & 17 & 1 \\
Mowable Areas & 64 & 35 & 1 \\
Warms Season & 76 & 24 & 0 \\
Cool Season & 87 & 13 & 0 \\
High Elevation & 96 & 4 & 0 \\
\hline
\end{tabular}

Table 49: Objective 1 Percent by Species Data at 90 Days ( $n=3$ for each treatment)

\begin{tabular}{lccc}
\hline & $\begin{array}{c}\text { Mean } \\
\text { Planted }\end{array}$ & $\begin{array}{c}\text { Mean Not } \\
\text { Planted }\end{array}$ & $\begin{array}{c}\text { Mean No } \\
\text { Cover }\end{array}$ \\
\hline DOH Medians & 69 & 31 & 0 \\
DOH Cut/Fill & 65 & 35 & 0 \\
Mowable Areas & 62 & 38 & 0 \\
Warms Season & 64 & 36 & 0 \\
Cool Season & 75 & 25 & 0 \\
High Elevation & 88 & 12 & 0 \\
\hline
\end{tabular}




\subsection{Percent Cover Data for Objective 2}

Table 50: Objective 2 Percent Cover Data Over 90 Days ( $n=3$ for each treatment)

\begin{tabular}{lccccccc}
\hline $\begin{array}{l}\text { Data } \\
\text { Collection } \\
\text { Date }\end{array}$ & $\mathbf{6 / 3 / 1 5}$ & $\mathbf{6 / 1 9 / 1 5}$ & $\mathbf{7 / 1 / 1 5}$ & $\mathbf{7 / 1 6 / 1 5}$ & $\mathbf{7 / 2 8 / 1 5}$ & $\mathbf{8 / 1 1 / 1 5}$ & $\mathbf{8 / 2 4 / 1 5}$ \\
\hline $\begin{array}{l}\text { DOH } \\
\text { Medians }\end{array}$ & 3.7 & 58.3 & 66.7 & 72.0 & 66.7 & 72.3 & 89.0 \\
$\begin{array}{l}\text { Mowable } \\
\text { Areas }\end{array}$ & 1.7 & 72.3 & 84.0 & 87.3 & 82.7 & 84.0 & 97.0 \\
$\begin{array}{l}\text { Warm } \\
\text { Season }\end{array}$ & 0.0 & 20.3 & 26.7 & 32.7 & 22.7 & 30.7 & 62.7 \\
$\begin{array}{l}\text { Cool } \\
\text { Season }\end{array}$ & 4.3 & 43.7 & 59.3 & 81.7 & 83.3 & 87.7 & 95.0 \\
$\begin{array}{l}\text { High } \\
\text { Elevation }\end{array}$ & 1.3 & 30.0 & 32.7 & 28.7 & 22.0 & 34.3 & 58.3 \\
\hline
\end{tabular}

Table 51: Objective 2 Percent by Species Data at 51 Days ( $n=3$ for each treatment)

\begin{tabular}{lccc}
\hline & $\begin{array}{c}\text { Mean } \\
\text { Planted }\end{array}$ & $\begin{array}{c}\text { Mean Not } \\
\text { Planted }\end{array}$ & $\begin{array}{c}\text { Mean No } \\
\text { Cover }\end{array}$ \\
\hline DOH Medians & 81 & 2 & 17 \\
Mowable Areas & 87 & 1 & 12 \\
Warm Season & 44 & 8 & 48 \\
Cool Season & 84 & 1 & 15 \\
High Elevation & 48 & 6 & 46 \\
\hline
\end{tabular}

Table 52: Objective 2 Percent by Species Data at 90 days ( $n=3$ for each treatment)

\begin{tabular}{lccc}
\hline & $\begin{array}{c}\text { Mean } \\
\text { Planted }\end{array}$ & $\begin{array}{c}\text { Mean Not } \\
\text { Planted }\end{array}$ & $\begin{array}{c}\text { Mean No } \\
\text { Cover }\end{array}$ \\
\hline DOH Medians & 86 & 1 & 13 \\
Mowable Areas & 90 & 0 & 10 \\
Warm Season & 19 & 25 & 55 \\
Cool Season & 62 & 27 & 11 \\
High Elevation & 19 & 32 & 50 \\
\hline
\end{tabular}




\subsection{Percent Cover Data for Objective 3}

Table 53: Objective 3 Percent Cover Over 90 Days ( $n=2$ for each treatment)

\begin{tabular}{|c|c|c|c|c|c|c|c|}
\hline $\begin{array}{l}\text { Data } \\
\text { Collection } \\
\text { Date } \\
\end{array}$ & $6 / 3 / 15$ & $6 / 19 / 15$ & $7 / 1 / 15$ & $7 / 16 / 15$ & $7 / 28 / 15$ & $8 / 11 / 15$ & $8 / 24 / 15$ \\
\hline & \multicolumn{7}{|c|}{ Mean Percent Cover } \\
\hline $\begin{array}{l}\text { DOH } \\
\text { Medians } \\
\text { (TS, HP) }\end{array}$ & 13.0 & 75.0 & 85.0 & 98.0 & 98.0 & 99.0 & 97.5 \\
\hline $\begin{array}{l}\text { DOH } \\
\text { Medians } \\
\text { (NTS, HP) }\end{array}$ & 12.0 & 83.0 & 92.5 & 95.5 & 96.5 & 97.5 & 94.5 \\
\hline $\begin{array}{l}\text { DOH } \\
\text { Medians } \\
\text { (TS,S) }\end{array}$ & 5.0 & 50.5 & 74.5 & 90.0 & 92.5 & 95.5 & 95.0 \\
\hline $\begin{array}{l}\text { DOH } \\
\text { Medians } \\
\text { (NTS,S) }\end{array}$ & 3.0 & 77.5 & 90.0 & 93.5 & 93.5 & 89.5 & 95.5 \\
\hline $\begin{array}{l}\text { Warm } \\
\text { Season } \\
\text { (TS, HP) }\end{array}$ & 0.5 & 38.5 & 65.5 & 91.0 & 95.0 & 95.0 & 97.0 \\
\hline $\begin{array}{l}\text { Warm } \\
\text { Season } \\
\text { (NTS, HP) }\end{array}$ & 1 & 50.5 & 81.5 & 80.5 & 79.5 & 81.5 & 91 \\
\hline $\begin{array}{l}\text { Warm } \\
\text { Season } \\
(\mathrm{TS}, \mathrm{S})\end{array}$ & 0 & 20.5 & 44.5 & 87 & 91 & 86 & 93 \\
\hline $\begin{array}{l}\text { Warm } \\
\text { Season } \\
\text { (NTS,S) }\end{array}$ & 2 & 55 & 80 & 89 & 81 & 92 & 95 \\
\hline $\begin{array}{l}\text { Cool } \\
\text { Season } \\
\text { (TS, HP) }\end{array}$ & 12.5 & 36 & 57 & 95 & 91 & 96 & 97 \\
\hline $\begin{array}{l}\text { Cool } \\
\text { Season } \\
\text { (NTS, HP) }\end{array}$ & 7 & 61.5 & 80 & 93 & 98 & 98.5 & 98.5 \\
\hline $\begin{array}{l}\text { Cool } \\
\text { Season } \\
(\mathrm{TS}, \mathrm{S})\end{array}$ & 3 & 35.5 & 45.5 & 75 & 92 & 92.5 & 97.5 \\
\hline $\begin{array}{l}\text { Cool } \\
\text { Season } \\
\text { (NTS,S) }\end{array}$ & 2 & 59.5 & 77.5 & 91.5 & 90 & 88 & 91.5 \\
\hline
\end{tabular}


Table 54: Objective 3 Percent by Species Data at 51 Days ( $n=2$ for each treatment)

\begin{tabular}{lccc}
\hline & $\begin{array}{c}\text { Mean } \\
\text { Planted }\end{array}$ & $\begin{array}{c}\text { Mean Not } \\
\text { Planted }\end{array}$ & $\begin{array}{c}\text { Mean No } \\
\text { Cover }\end{array}$ \\
\hline DOH Medians (TS, HP) & 82 & 15 & 4 \\
DOH Medians (TS, S) & 64 & 25 & 11 \\
DOH Medians (NTS, HP) & 89 & 2 & 10 \\
DOH Medians (NTS, S) & 87 & 3 & 10 \\
Warm Season (TS, HP) & 53 & 36 & 12 \\
Warm Season (TS, S) & 28 & 53 & 20 \\
Warm Season (NTS, HP) & 85 & 0 & 15 \\
Warm Season (NTS, S) & 76 & 8 & 17 \\
Cool Season (TS, HP) & 78 & 13 & 10 \\
Cool Season (TS, S) & 36 & 30 & 35 \\
Cool Season (NTS, HP) & 77 & 14 & 10 \\
Cool Season (NTS, S) & 48 & 35 & 18 \\
\hline
\end{tabular}

Table 55: Objective 3 Percent by Species Data at 90 Days ( $n=2$ for each treatment)

\begin{tabular}{lccc}
\hline & $\begin{array}{c}\text { Mean } \\
\text { Planted }\end{array}$ & $\begin{array}{c}\text { Mean Not } \\
\text { Planted }\end{array}$ & $\begin{array}{c}\text { Mean No } \\
\text { Cover }\end{array}$ \\
\hline DOH Medians (TS, HP) & 17 & 82 & 2 \\
DOH Medians (TS, S) & 17 & 84 & 0 \\
DOH Medians (NTS, HP) & 90 & 8 & 2 \\
DOH Medians (NTS, S) & 97 & 1 & 3 \\
Warm Season (TS, HP) & 22 & 73 & 5 \\
Warm Season (TS, S) & 42 & 41 & 18 \\
Warm Season (NTS, HP) & 87 & 0 & 13 \\
Warm Season (NTS, S) & 87 & 0 & 13 \\
Cool Season (TS, HP) & 49 & 49 & 3 \\
Cool Season (TS, S) & 37 & 57 & 7 \\
Cool Season (NTS, HP) & 79 & 17 & 5 \\
Cool Season (NTS, S) & 84 & 8 & 8 \\
\hline
\end{tabular}




\subsection{Percent Cover Data for Objective 4}

Table 56: Objective 4 Percent Cover Over 90 Days ( $n=3$ for each treatment)

\begin{tabular}{|c|c|c|c|c|c|c|c|}
\hline $\begin{array}{l}\text { Data } \\
\text { Collection } \\
\text { Date } \\
\end{array}$ & $6 / 3 / 15$ & $6 / 19 / 15$ & $7 / 1 / 15$ & $7 / 16 / 15$ & $7 / 28 / 15$ & $8 / 11 / 15$ & $8 / 24 / 15$ \\
\hline & \multicolumn{7}{|c|}{ Mean Percent Cover } \\
\hline Topsoil & 6.3 & 69.3 & 84.0 & 100.0 & 100.0 & 100.0 & 99.7 \\
\hline $\begin{array}{l}\text { No } \\
\text { Topsoil }\end{array}$ & 5.7 & 83.0 & 90.7 & 98.7 & 98.7 & 97.7 & 99.0 \\
\hline $\begin{array}{l}\text { Biotic } \\
\text { Earth }\end{array}$ & 3.3 & 69.3 & 88.0 & 95.3 & 97.3 & 96.7 & 98.7 \\
\hline ProGanics & 2.7 & 82.0 & 88.3 & 97.0 & 97.7 & 96.7 & 96.7 \\
\hline
\end{tabular}

Table 57: Objective 4 Percent by Species Data at 51 Days ( $n=3$ for each treatment)

\begin{tabular}{lccc}
\hline & $\begin{array}{c}\text { Mean } \\
\text { Planted }\end{array}$ & $\begin{array}{c}\text { Mean Not } \\
\text { Planted }\end{array}$ & $\begin{array}{c}\text { Mean No } \\
\text { Cover }\end{array}$ \\
\hline Topsoil & 3 & 96 & 0 \\
No Topsoil & 79 & 15 & 5 \\
ProGanics & 92 & 3 & 5 \\
$\begin{array}{l}\text { Biotic Earth } \\
\text { Black }\end{array}$ & 84 & 9 & 7 \\
\hline
\end{tabular}

Table 58: Objective 4 Percent by Species Data at 90 Days ( $n=3$ for each treatment)

\begin{tabular}{lccc}
\hline & $\begin{array}{c}\text { Mean } \\
\text { Planted }\end{array}$ & $\begin{array}{c}\text { Mean Not } \\
\text { Planted }\end{array}$ & Mean No Cover \\
\hline Topsoil & 0 & 100 & 0 \\
No Topsoil & 78 & 16 & 6 \\
ProGanics & 83 & 9 & 8 \\
$\begin{array}{l}\text { Biotic Earth } \\
\text { Black }\end{array}$ & 72 & 18 & 10 \\
\hline
\end{tabular}

